\title{
Impedance-Based Structural Health Monitoring of Wind Turbine Blades
}

\author{
by \\ Corey Wilson Pitchford
}

Thesis submitted to the faculty of the Virginia Polytechnic Institute and State University in partial fulfillment of the requirements for the degree of

Master of Science

in

Mechanical Engineering

\author{
Dr. Daniel J. Inman, Chair \\ Dr. Nakhiah Goulbourne \\ Dr. Martin E. Johnson \\ Dr. Daniel M. Peairs
}

August 31, 2007

Blacksburg, Virginia

Keywords: structural health monitoring, impedance method, wind turbines, wind turbine blades

Copyright 2007, Corey Pitchford 


\title{
Impedance-Based Structural Health Monitoring of Wind Turbine Blades
}

\author{
Corey Wilson Pitchford
}

\begin{abstract}
Wind power is a fast-growing source of non-polluting, renewable energy with vast potential. However, current wind technology must be improved before the potential of wind power can be fully realized. One of the key components in improving wind turbines is their blades. Blade failure is very costly because blade failure can damage other blades, the wind turbine itself, and possibly other wind turbines. A successful structural health monitoring (SHM) system incorporated into wind turbines could extend blade life and allow for less conservative designs.
\end{abstract}

Impedance-based SHM is a method which has shown promise on a wide variety of structures. The technique utilizes small piezoceramic (PZT) patches attached to a structure as self-sensing actuators to both excite the structure with high-frequency excitations, and monitor any changes in structural mechanical impedance. By monitoring the electrical impedance of the PZT, assessments can be made about the integrity of the mechanical structure. Recent advances in hardware systems with onboard computing, including actuation and sensing, computational algorithms, and wireless telemetry, have improved the accessibility of the impedance method for in-field measurements.

The feasibility of implementing impedance-based SHM on wind turbine blades is investigated in this work. Experimentation was performed to determine the capability of the method to detect damage on blades. First, tests were run to detect both indirect and actual forms of damage on a section of an actual wind turbine blade provided by Sandia National Laboratories. Additional tests were run on the same blade section using a high-frequency response function method of SHM for comparison. Finally, based on the results of the initial testing, the impedance method was utilized in an attempt to detect damage during a fatigue test of an experimental wind turbine blade at the National Renewable Energy Laboratory's National Wind Technology Center. 


\section{ACKNOWLEDGEMENTS}

First, I would like to express my gratitude to my advisor, Dr. Inman, for taking a student who was a little unsure of what he wanted to do in grad school and giving me the opportunity to work in CIMSS. For this I am very grateful, and I am as well for his advice and patience throughout this research.

I would like to thank Mark Rumsey of Sandia National Laboratories for the opportunities to test the $\mathrm{CX}-100$ blade section and to participate in the TX-100 fatigue test as well as his assistance throughout this research. Thank you also to Joshua Paquette of Sandia and Jeroen van Dam, Scott Hughes, and Mike Jenks of the National Wind Technology Center for your help and guidance during the blade fatigue test. Thank you as well to Robert Owen of Extreme Diagnostics for his participation in this research and to Ben Grisso, Gyuhae Park, and Dan Peairs for their guidance and recommendations.

Thank you to all of my friends at Virginia Tech and in CIMSS. This has been an amazing time that I will never forget thanks to you all.

I would like to thank my parents for the encouragement and interest they have always shown in everything that I have done and for always letting me know how proud they are of me.

Finally, I would like to thank my wife, Melissa, for her unwavering love and support throughout this experience. I could not have accomplished this without being able to spend time with you each day or without your ability to help me see the big picture during the difficult times. 


\section{TABLE OF CONTENTS}

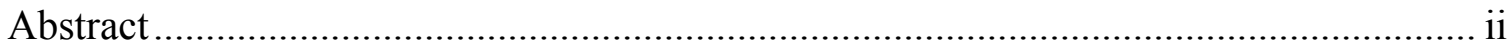

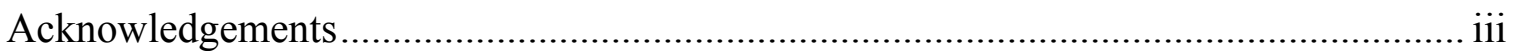

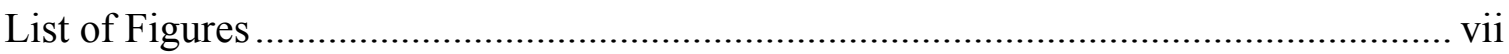

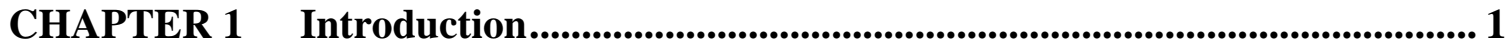

1.1 Wind power................................................................................................ 1

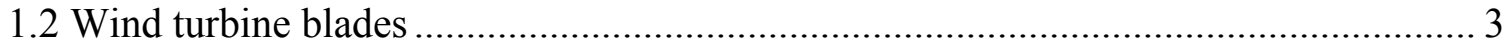

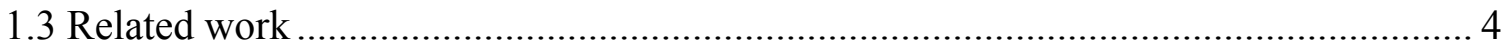

1.4 Impedance-based structural health monitoring ........................................................ 6

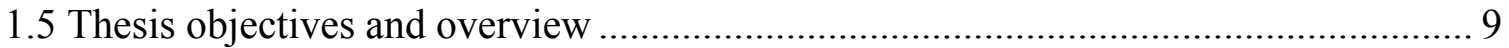

\section{CHAPTER 2 Impedance-Based Structural Health Monitoring of CX-100 Wind}

Turbine Blade Section ............................................................................................ 11

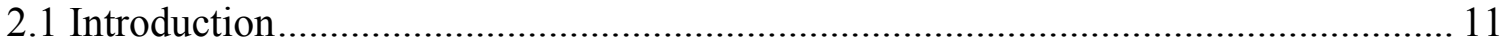

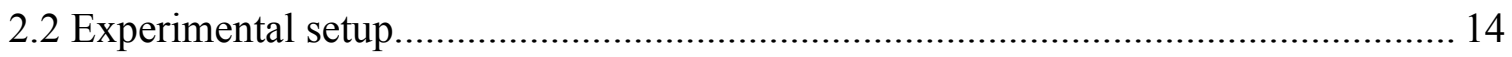

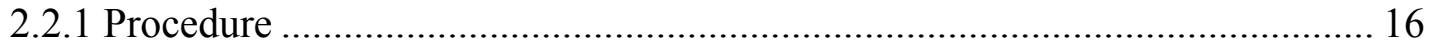

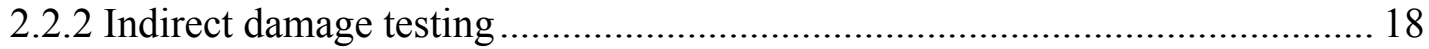

2.2.3 Actual damage testing.................................................................................. 19

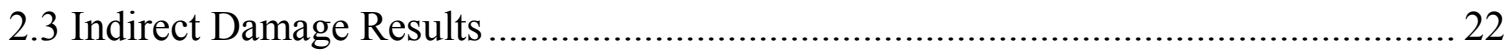

2.3.1 Added mass results ................................................................................ 24

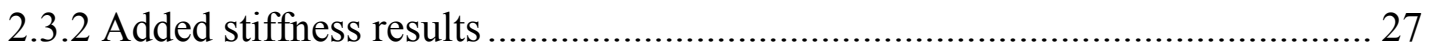

2.3.3 Added stiffness, higher frequency results .................................................. 29

2.3.4 Moving mass results .................................................................................... 31

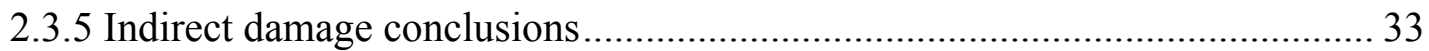

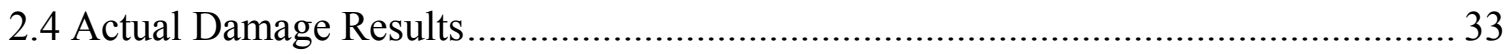

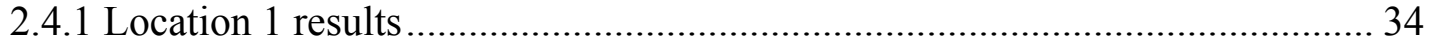

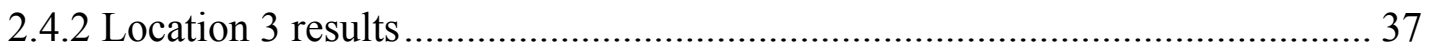

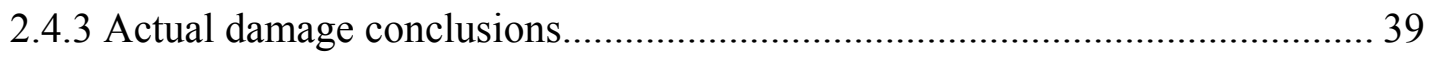

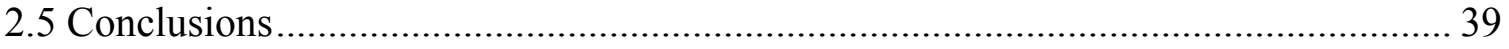




\section{CHAPTER 3 High-Frequency Response Function Structural Health Monitoring}

of CX-100 Wind Turbine Blade Section ........................................................................ 41

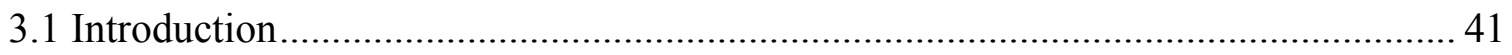

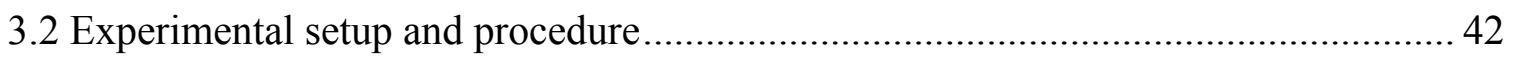

3.3 Indirect damage results ....................................................................................... 43

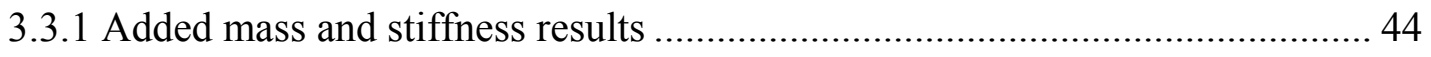

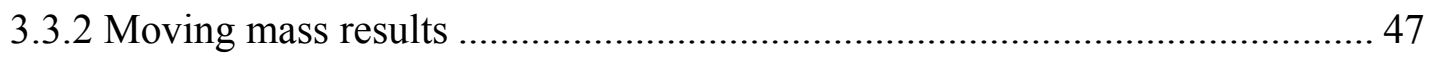

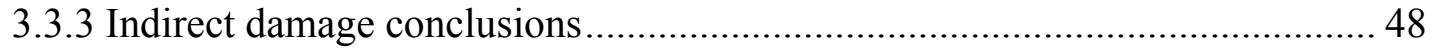

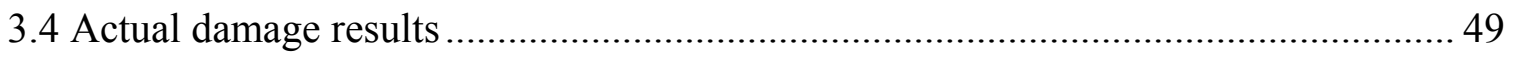

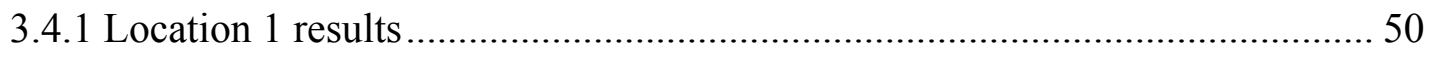

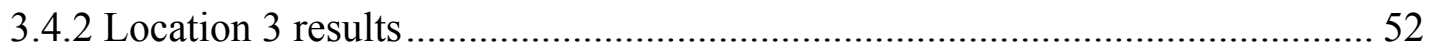

3.4.3 Actual damage conclusions........................................................................ 53

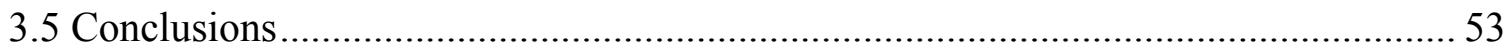

\section{CHAPTER 4 Impedance-Based Structural Health Monitoring of TX-100 Wind}

Turbine Blade Fatigue Test.......................................................................................................... 55

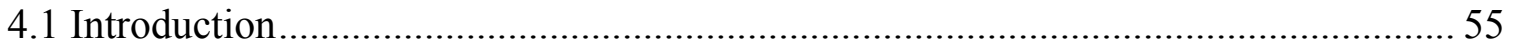

4.2 Preliminary tests on CX-100 wind turbine blade section .............................................6 60

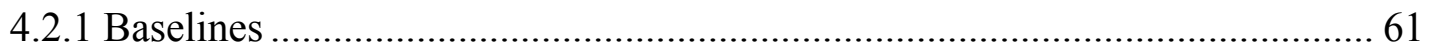

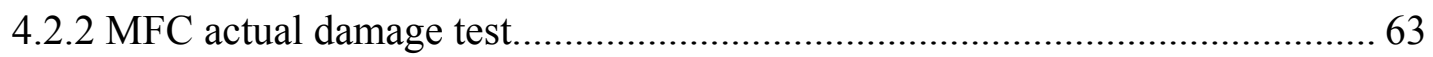

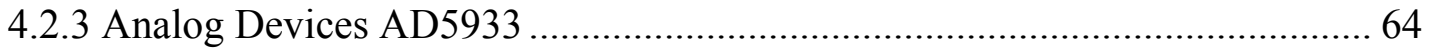

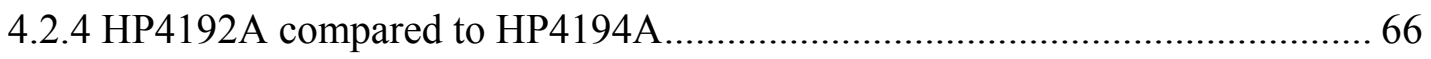

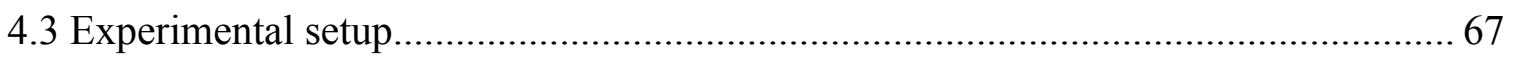

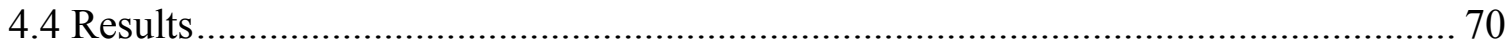

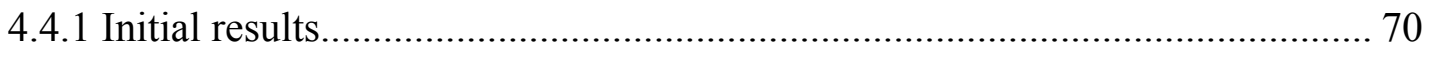

4.4.2 During fatigue test operation ..................................................................... 71

4.4.3 Data collection and baseline damage metrics ................................................ 72

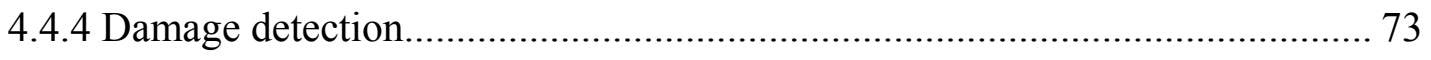

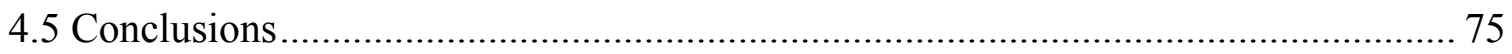


CHAPTER 5 Summary and Conclusions....................................................................... 76

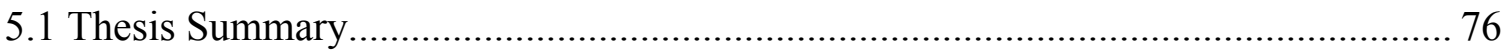

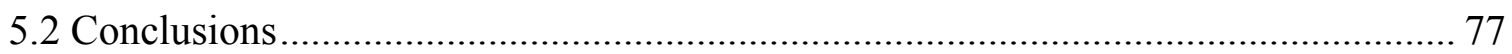

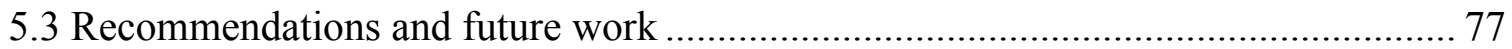

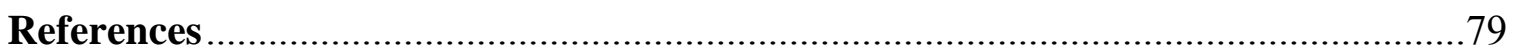

APPENDIX A: IBSHM, indirect damage, impedance plots ......................................86

APPENDIX B: IBSHM, actual damage, impedance plots ........................................95

APPENDIX C: HFRF, indirect damage, transfer functions......................................99

APPENDIX D: HFRF, indirect damage, transfer functions.....................................102

APPENDIX E: HP4194A/HP4192A comparison, impedance plots …………….......104

APPENDIX F： Fatigue test initial impedance plots................................................106

APPENDIX G: Data collection instructions for TX-100 fatigue test impedance

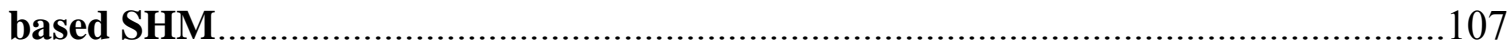

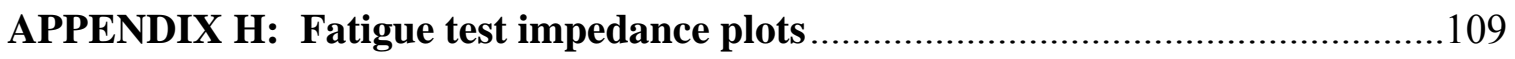

Vita 


\section{LIST OF FIGURES}

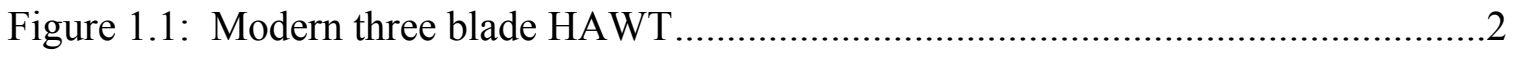

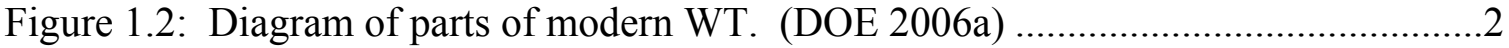

Figure 1.3: Installed wind power capacity in United States 1981-2007. Data from (DOE 2007c).

Figure 1.4: Model used to derive electromechanical admittance of PZT bonded to structure.

Figure 2.1: CX-100 high pressure skin showing carbon fiber spar cap infused with typical glass/balsa skin. (Paquette et al. 2006) .12

Figure 2.2: Overall picture of TX-100 blade. (Paquette et al. 2006).............................12

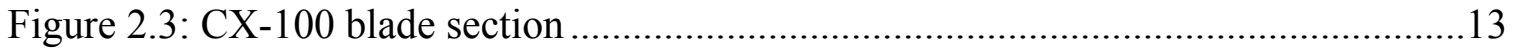

Figure 2.4: CX-100 Cross-section with damage modes .............................................14

Figure 2.5: Sensor names and locations (damage locations shown for reference) ...........15

Figure 2.6: Removed adhesive for carbon fiber pzt................................................ 15

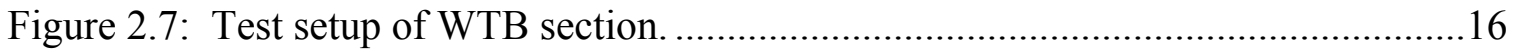

Figure 2.8: Indirect damage methods: Added mass (25g magnets) in location 1 and added stiffness (C-clamp) in location 2. ................................................ 18

Figure 2.9: Additional (225g) mass simulated damage at location 2 ............................19

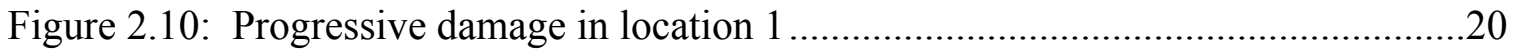

Figure 2.11: Damage in location 1 located down the length of the blade .......................21

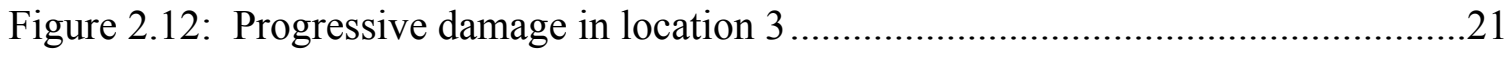

Figure 2.13: Damage in location 3 located down the length of the blade ......................22

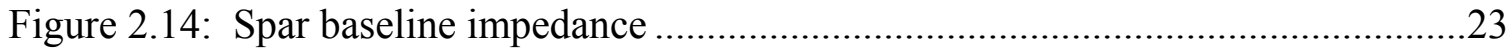

Figure 2.15: Impedance plot for spar sensor, 10-30 kHz, added mass ..........................24

Figure 2.16: Impedance plot for spar sensor, 10-30 kHz, added stiffness (C-clamp) ......24

Figure 2.17: Skin: Added mass (25g): Damage metrics calculated for several frequency

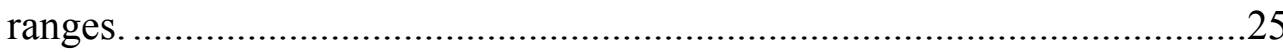

Figure 2.18: Spar: Added mass (25 g): Damage metrics calculated for several frequency ranges. .25 
Figure 2.19: Carbon: Added mass (25 g): Damage metrics calculated for several frequency ranges. .26

Figure 2.20: Skin, clamp: Damage metrics calculated for several frequency ranges......27

Figure 2.21: Spar, clamp: Damage metrics calculated for several frequency ranges. .....28

Figure 2.22: Carbon, clamp: Damage metrics calculated for several frequency ranges..28

Figure 2.23: Skin, clamp: Damage metrics calculated for several frequency ranges......29

Figure 2.24: Spar, clamp: Damage metrics calculated for several frequency ranges. .....30

Figure 2.25: Carbon, clamp: Damage metrics calculated for several frequency ranges..30

Figure 2.26: Spar, moving mass: Test 1, Damage metrics calculated for several frequency ranges.

Figure 2.27: Spar, moving mass: Test 2, Damage metrics calculated for several frequency ranges.

Figure 2.28: Carbon, moving mass: Damage metrics calculated for several frequency ranges.

Figure 2.29: Impedance plot for skin sensor, 10-30 kHz, location 1 sensor end progressive damage.

Figure 2.30: Impedance plot for carbon sensor, $10-30 \mathrm{kHz}$, location 3 sensor end

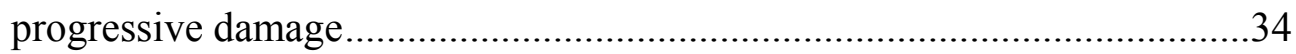

Figure 2.31: Damage metrics for location 1 actual damage tests, $10-30 \mathrm{kHz}$..................35

Figure 2.32: Damage metrics for location 1 actual damage tests, $30-60 \mathrm{kHz}$..................35

Figure 2.33: Damage metrics for location 1 actual damage tests, 30-60 kHz. Enhanced

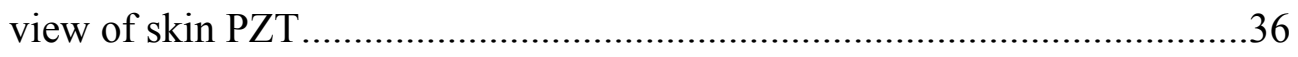

Figure 2.34: Damage metrics for location 3 actual damage tests, $10-30 \mathrm{kHz}$.................37

Figure 2.35: Damage metrics for location 3 actual damage tests, $30-60 \mathrm{kHz}$..................38

Figure 2.36: Damage metrics for location 3 actual damage tests, 30-60 kHz. Enhanced view for the spar PZT .38

Figure 3.1: Baseline transfer function and coherence, skin/carbon PZTs, 1V random signal input.

Figure 3.2: Spar/Carbon transfer function, $1 \mathrm{~V}$ random signal, added stiffness .45

Figure 3.3: Skin/Carbon transfer function, $5 \mathrm{~V}$ chirp signal, added mass...... .45 
Figure 3.4: Added mass (25g) and stiffness (C-clamp) at sensor end. 1V random signal input

Figure 3.5: Added mass (25g) and stiffness (C-clamp) at sensor end. 5V chirp signal input .46

Figure 3.6: Moving mass results. 1V random signal input. .47

Figure 3.7: Moving mass results. 5V chirp signal input. .48

Figure 3.8: Spar/carbon PZT combination, location 1 split at sensor end 49

Figure 3.9: Skin/carbon PZT combination, location 3 holes at sensor end .50

Figure 3.10: Damage metrics for location 1 actual damage HFRF test .51

Figure 3.11: Damage metrics for location 1 actual damage HFRF test, enhanced view of skin/spar PZT combination .51

Figure 3.12: Damage metrics for location 3 actual damage HFRF test .52

Figure 4.1: TX-100 HP and LP skins showing implementation of carbon fiber into design. (Paquette et al. 2006)................................................................56

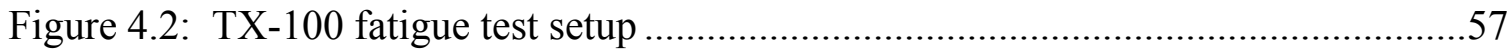

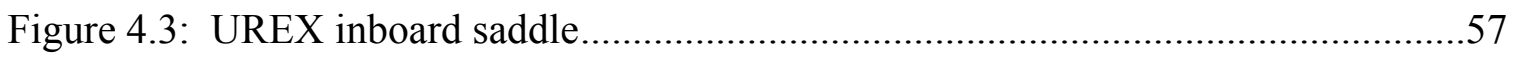

Figure 4.4: Smart Material MFC, model number M2814 P1, 28x14mm active area, $37 \times 17 \mathrm{~mm}$ overall

Figure 4.5: Analog Devices AD5933 impedance chip and evaluation board. Evaluation board 100x80mm and impedance chip (circled) $5 \times 5 \mathrm{~mm}$. .60

Figure 4.6: MFC locations on CX-100 WTB section 61

Figure 4.7: Baseline impedance for inner and outer LP MFC. .62

Figure 4.8: Baseline impedance for inner and outer HP MFC .62

Figure 4.9: Impedance plot for LP inner MFC, progressively larger holes in adhesive...63 Figure 4.10: Impedance plot for LP outer MFC, progressively larger holes in adhesive.64 Figure 4.11: Damage metrics for LP inner and outer MFC, progressively larger holes in adhesive....... .64

Figure 4.12: Magnitude and phase of baseline impedance for 4 MFCs: AD5933. .........65

Figure 4.13: Magnitude and phase of baseline impedance for 4 MFCs: HP4194A. .......66

Figure 4.14: Damage metrics comparing HP4194A and HP4192A results of indirect damage test using MFCs 
Figure 4.15: IBSHM measurement setup for TX-100 fatigue test. Laptop, HP4192A impedance analyzer and switch box.

Figure 4.16: Switch box to input 6 MFCs into HP4192A for TX-100 fatigue test. .........68

Figure 4.17: Layout of MFCs for IBSHM on TX-100 schematic.

Figure 4.18: Picture of MFC layout on downward facing (LP) side of TX-100. Also shown are sensors for other damage detection methods.

Figure 4.19: Baseline impedance plot for s3 MFC, 5-60 kHz ..................................... 70

Figure 4.20: Impedance signature of s3 MFC, both with fatigue test stopped and running illustrating "beat" frequency

Figure 4.21: Graph of fatigue test cycle count showing where baseline measurements took place. .72

Figure 4.22: Baseline damage metrics for fatigue test for 6 MFCs shown with resulting damage thresholds.

Figure 4.23: Damage metrics for fatigue test plotted versus cycle count and shown with damage threshold. 


\section{CHAPTER 1 Introduction}

\subsection{Wind power}

Wind power is a source of non-polluting, renewable energy. Using wind as an energy source is not a new technology as the first reliable source of the existence of windmills dates back to 644 A.D. and some have claimed to have found remains of windmills 3000 years old. From that time windmills were used to mill grain and later pump water. In the early 1900s, wind was first used to generate electricity (Hau 2006).

Today, wind turbines (WT) can generate megawatts of power and have rotor diameters that are on the order of 100 meters in diameter. Modern WT, like the one shown in Figure 1.1, are nearly all horizontal-axis WT (HAWT) and have three rotor blades. The components of a modern WT can be seen in Figure 1.2. The nacelle sits on top of a tall tower and houses the shaft coming from the rotor, a gearbox, and the generator. The nacelle and rotor can yaw on the tower so that the rotor faces into the wind. The blades of modern WT are manufactured borrowing technology from the boat building industry, are made of both glass and wood composites, and have an airfoil shape adapted from aeronautical engineering (Hau 2006). The blades have the ability to pitch in the rotor hub to maintain the optimal angle of attach given the conditions as well as to pitch far enough to stop the rotor in extreme conditions. Some turbines also have internal brakes to stop the rotor as shown in the illustration. 


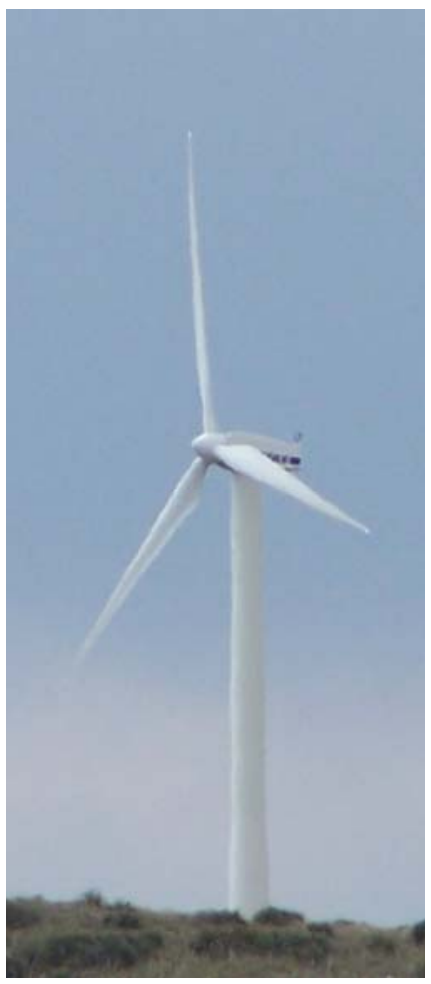

Figure 1.1: Modern three blade HAWT

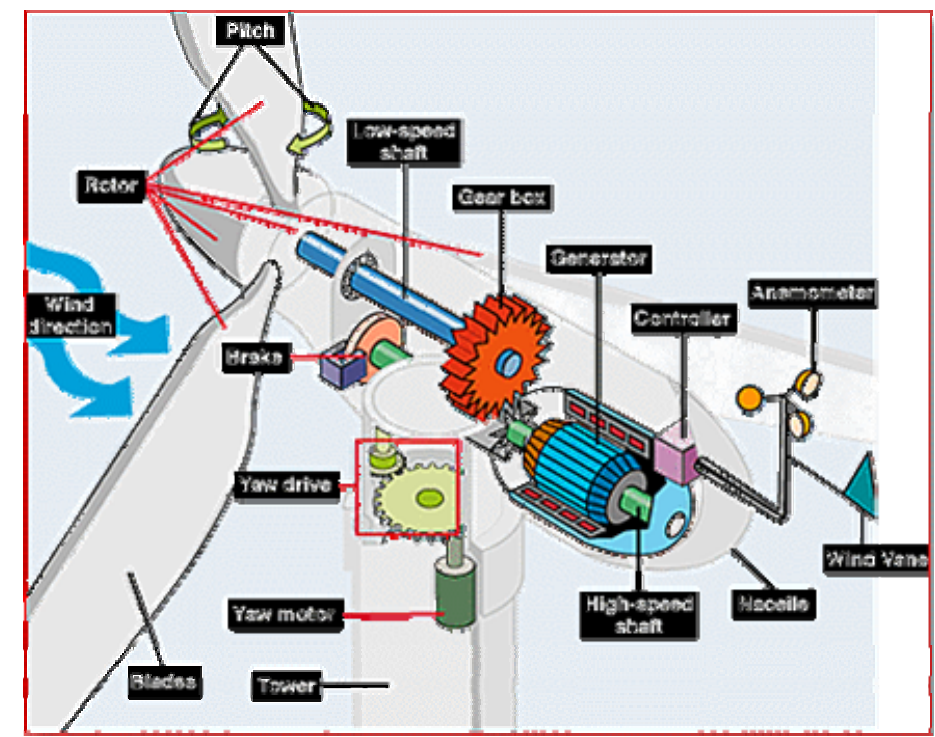

Figure 1.2: Diagram of parts of modern WT. (DOE 2006a)

Wind power is one of the fastest growing energy technologies in the world. Last year, wind power generation in the United States grew 27\% and it is expected to grow 26\% in 2007 (AWEA 2007a). Wind power has been the second largest source of new power generation in the country for the past two years second only to natural gas (AWEA 
2007b). Figure 1.3 illustrates the drastic increase in wind power capacity in the United States in recent years, but wind still only currently makes $1 \%$ of the country's total electric generation capacity (DOE 2006b). However, the wind resource is vast and relatively untapped in the United States and theoretically could supply all of the nation's energy needs (AWEA 2004). Wind as a sole power source is of course a hypothetical possibility, but the federal government has made a goal for $20 \%$ of the country's power to be supplied by wind (DOE 2006b). However, for this goal to become a reality, WT technology must be improved. The Department of Energy's Wind Energy Program works with the WT industry and research labs, like Sandia National Laboratories (SNL) and the National Renewable Energy Laboratory's (NREL) National Wind Technology Center (NWTC), to improve wind energy technology.

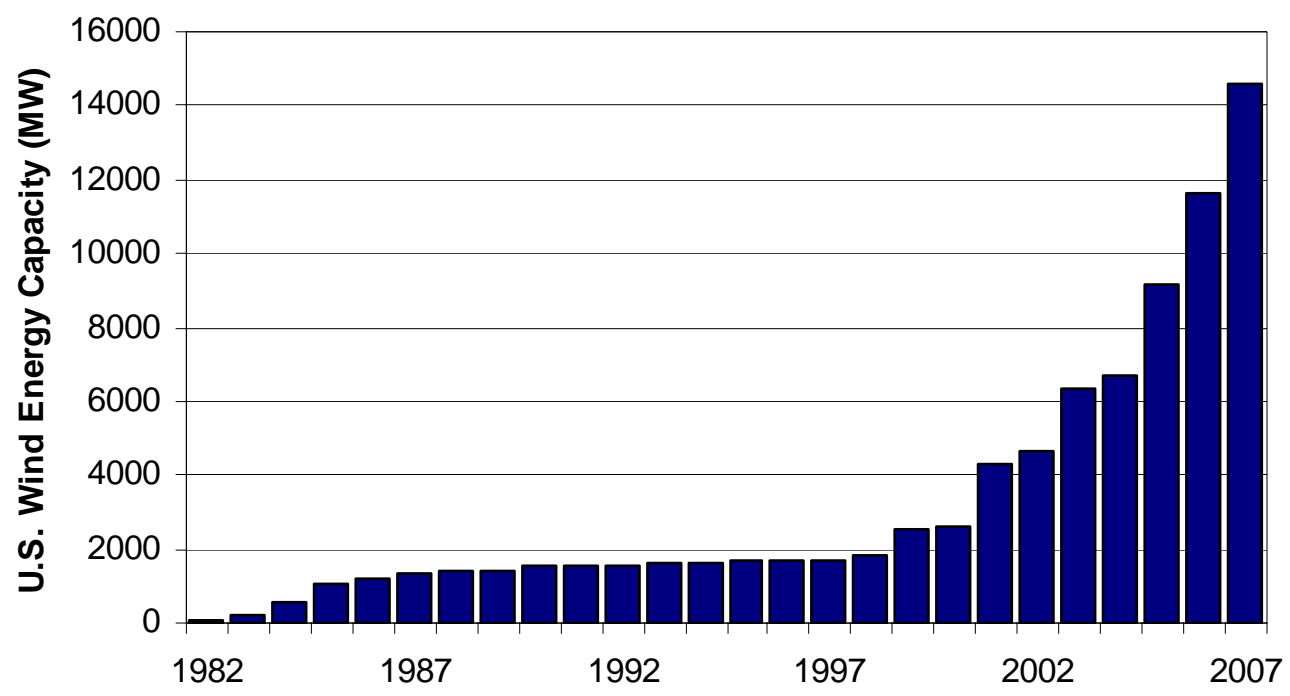

Figure 1.3: Installed wind power capacity in United States 1981-2007. Data from (DOE 2007c).

\subsection{Wind turbine blades}

Wind turbine blades (WTB) are especially important in the development of wind power technology. The blades are the only part of the turbine designed specifically for the wind energy industry and represent the most difficult design challenge for the entire turbine. "The rotor blades are considered to be the key component for the further 
technical development of the entire wind turbine system" (Hau 2006). In order to expand WT technology, blades must have increased performance while maintaining low cost. To increase the effectiveness of WTs, blade designs have grown longer and current wind turbine designs have rotor diameters on the order of 100 meters. At the same time WTBs are made from lighter more cost effective materials such as fiberglass and/or other composites. These extremely large composite structures undergo a complex load spectrum with a high number of cycles, and are clearly fatigue critical machines (Sutherland 1999).

These design challenges contribute to the concern for blade failure which also can damage other parts of the WT and/or other nearby WTs (Simmermacher et al. 1997). Although advances have been made in predicting the fatigue life of blades (Sutherland 1999), they can only help in predicting the service life of blades, not the instant they will fail. Therefore blades would certainly benefit from structural health monitoring (SHM), as blade life could be extended or blade designs made less conservative if a successful damage detection method could be implemented. Finally, WT are often in remote areas and, especially in Europe, often offshore. Inspection of offshore blades is difficult and would provide another advantage for a blade SHM system.

\subsection{Related work}

The need for damage detection in WTB has been recognized by the wind energy industry for some time, and several methods have been tested. Work at SNL and NREL in the 1990s included blade fatigue tests and field tests incorporating laser vibrometers and accelerometers in an attempt to detect damage with modal data (Gross et al. 1998; Rumsey et al. 1997; Simmermacher et al. 1997). Also tested were acoustic emissions (AE) and coherent optical/shearograhpy methods during quasi-static testing of a blade (Sutherland et al. 1993). AE was also used during blade fatigue tests along with infrared thermography (Beattie 1997; Beattie and Rumsey 1999). Finally, ultrasonic C-scan methods were investigated (Gieske and Rumsey 1997). Recent work from the European wind energy community is primarily in the area of condition monitoring of WT using 
global modal methods (Reetz 2007; Rolfes et al. 2006). These methods have been successful in detecting damage in WTB, but are mostly non-destructive evaluation tools that are not promising methods for on-board SHM. They also mainly utilize lowfrequencies which are good for detecting global types of damage, but not minor damage which could indicate imminent failure.

During the past several years, Sundaresan and Schulz et al. of North Carolina A\&T State University and the University of Cincinnati have partnered with NREL to perform research in the area of turbine blade SHM (Ghoshal et al. 2000; Kirikera et al. 2007; Schulz and Sundaresan 2006; Sundaresan et al. 2002; Sundaresan et al. 2001; Sundaresan et al. 1999). Their early work focused on low-frequency methods on a blade section using piezoceramic (PZT) actuators for actuation, laser vibrometers for sensing, and added mass for simulating damage. Next, they used stress waves transmitted and received with PZT patches to detect damage during a static WTB test at NREL. Most recently, they used PZTs to monitor AEs on a WTB during proof testing at NREL. Finally, they proposed a "smart" or "intelligent" blade which would have a network of sensors and be an autonomous system with on-board computing and power harvesting.

One of the more recent works in the area of WTB SHM was a collaborative effort between SNL and the NASA Kennedy Space Center (KSC). NASA KSC developed a macro-fiber composite (MFC) sensor/actuator which could potentially be used for SHM. The MFCs were tested on a CX-100 blade, the same model of experimental blade which was used in the work presented in Chapter 2, and also on a similar blade, 3X-100 during fatigue tests of the respective blades at NREL's NWTC. Large 57x86mm MFCs used as actuators and $38 \times 25 \mathrm{~mm}$ MFCs used as sensors were bonded to the blade near critical locations. The actuators sent out a random signal from $2-2500 \mathrm{~Hz}$ and transfer functions were measured from pairs of sensor MFCs. This method is somewhat similar to the one presented in Chapter 3, except that the method used by NASA is lower in frequency and it simply looks at the variance of each transfer function throughout the test. The method was somewhat successful in detecting damage in the $3 \mathrm{X}-100$ blade as variance of the transfer functions drops around the same time as damage occurs, but the results for the 
CX-100 are less discernible (Zayas et al. 2007). This method was also used during the fatigue test of a TX-100 blade, which is the work that is presented in Chapter 4.

\subsection{Impedance-based structural health monitoring}

The impedance-based SHM (IBSHM) method makes use of the electromechanical coupling of PZT patches. When a PZT is bonded to a structure it forms a collocated sensor and actuator, often referred to as a self-sensing actuator. When a voltage is applied across the PZT, the structure is displaced and conversely, when the structure is displaced a voltage is developed in the PZT. Therefore, the PZT can both actuate the surrounding area of the structure as well as sense the resulting structural response. If the PZT is driven with a sinusoidal voltage, this will cause the local area of the structure to vibrate and the structural response will cause an electrical response in the PZT. A one dimensional model of this setup is shown in Figure 1.4.

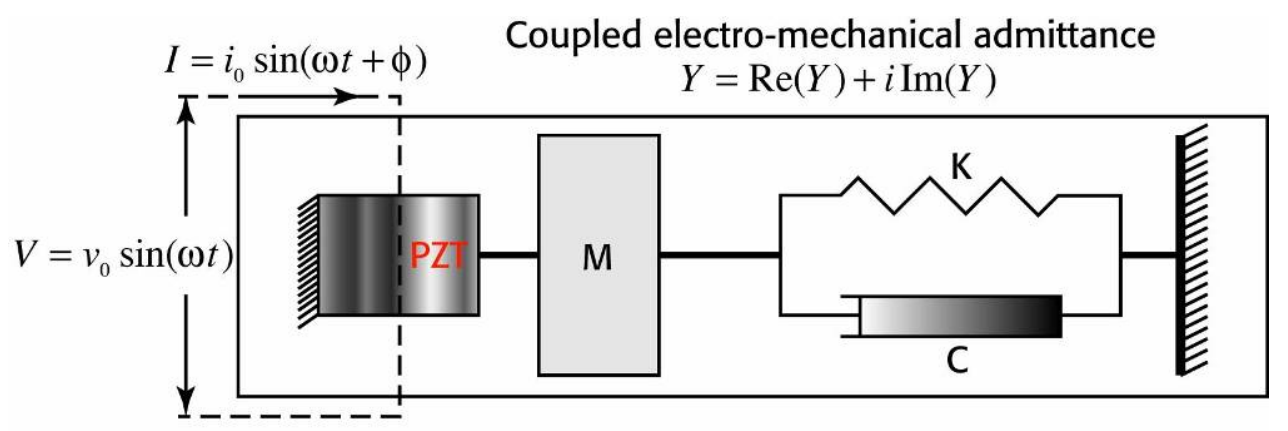

Figure 1.4: Model used to derive electromechanical admittance of PZT bonded to structure.

Using this model, it was originally shown analytically by Liang et al. (1994) that the electrical impedance (complex ratio of voltage to current) of the PZT is directly related to the local mechanical impedance (complex ratio of force to velocity) of the host structure. This is shown in the equation for the electrical admittance (inverse of impedance) of the bonded PZT derived by Liang,

$$
Y(\omega)=\frac{I}{V}=i \omega a\left(\bar{\varepsilon}_{33}{ }^{T}-\frac{Z_{s}(\omega)}{Z_{s}(\omega)+Z_{a}(\omega)} d_{3 x}{ }^{2} \hat{Y}_{x x}{ }^{E}\right)
$$


where $a$ is a piezoelectric geometric constant, $\bar{\varepsilon}_{33}{ }^{T}$ is the dielectric constant at zero stress, $d_{3 x}$ is the piezoelectric coupling constant in the $x$ direction at zero stress, $\hat{Y}_{x x}{ }^{E}$ is the complex Young's modulus of the PZT with zero electric field, and $Z_{a}$ and $Z_{s}$ are the PZT's and the structure's mechanical impedance, respectively. Given that the mechanical impedance and the material properties of the PZT stay constant, the equation shows that a change in the structure's mechanical impedance will directly result in a change in the electrical impedance of the PZT. Damage will cause a change in the structure's local mass, stiffness, or damping properties and consequently its mechanical impedance. Therefore, the structure's mechanical integrity can be assessed by monitoring the PZT's electrical impedance.

A review by Park et al (2003) summarizes the early work in this field to take the impedance method from Liang's early realization to a SHM method. This research established the parameters and methods that are typically used in IBSHM. The impedance of the PZT is typically measured over a frequency range of $30-400 \mathrm{kHz}$ using an excitation of 1 volt or less. This high structural frequency makes the method more sensitive to small damage and keeps it from being sensitive to boundary condition changes. The real part of the impedance is used for measurement because it was shown to be more sensitive to structural changes and less sensitive to temperature and other environmental effects. Frequency ranges are typically selected by trial and error, but those which contain 20-30 peaks in the real impedance are best to ensure sufficient structural information. In general, the larger the frequency range used, the smaller the area on the structure for sensing damage.

Once a proper frequency range is chosen, real impedance is measured on a frequency by frequency basis over the appropriate range to give an impedance "signature". First, several baseline impedance signatures are taken on the undamaged structure. Then impedance signatures are taken after the structure has been possibly damaged. To compare impedance signatures, change is quantified by a scalar damage 
metric. Peairs (2006) compares several damage metrics, but the most commonly used for the impedance method are root mean square deviation or cross-correlation. These metrics use the difference of the impedance at each frequency in calculating a scalar metric. Since there is change not associated with damage from one measurement to the next, a baseline metric is found by computing damage metrics for the undamaged impedance signatures. A threshold can also be established either experimentally or by looking at the variation of these baselines statistically. Finally, damage is indicated when a damage metric is computed for an impedance signature that is greater than the damage threshold.

Early research showed the method to be capable of detecting damage in a variety of structures including truss and bridge sections, an airplane tail, concrete walls, welded joints, and pipelines (Park 2003). More recently the impedance method has been shown to detect corrosion (Simmers 2005) and detect damage to thermal protection systems (Grisso and Inman 2006) and railroad track (Bouteiller et al. 2006). The method has also been shown to detect damage in composite materials. Grisso et al. showed the method to detect matrix cracking and delaminations on composite material samples (2004a; 2004b). Tarazaga et al. detected simulated orbital debris damage on a composite inflatable satellite boom using the impedance method (2006). The majority of the work investigating the impedance method has been on real world structures, but in a laboratory setting. One example of the few in-field tests on a real structure was conducted on space shuttle ground structures at KSC (Peairs et al. 2004).

Much of the research involving the impedance method has focused on making improvements that will allow it to be implemented on real working structures. Techniques have been developed to make impedance measurements insensitive to temperature variation (Koo et al. 2007; Park 1999). Impedance is traditionally measured for laboratory experiments with a bulky and expensive impedance analyzer but Peairs et al. developed a low-cost method for measuring impedance (2004). PZT material has been traditionally used as the self-sensing actuator, but it was shown that MFC actuators which are more flexible and durable could be used for the impedance method (Sodano 
2003). While the impedance method is a non-model based form of SHM, a modeling approach for the method would improve the ability for damage prognosis and optimizing system parameter. A spectral element model was developed for the method by Peairs (2006). Peairs et al. also found that when selecting a frequency range, if the range contains the resonance peaks of the free PZT or MFC, then damage detection sensitivity is increased (2006). Finally, Park et al. have developed a method using the imaginary part of the PZT's admittance to discern debonding and sensor damage from actual damage to the host structure (2006a).

Recently, work on the impedance method has focused on making the impedance measurement and accompanying computation system self-contained in one miniaturized and autonomous package. As previously mentioned, a bulky impedance analyzer has been traditionally used for taking laboratory measurements for the impedance method. The vision for an in-field system is that data processing, storage, and analysis is performed at the location of the sensor and the hardware systems that accomplish this would all be on one printed circuit board. Then wireless communication would transmit the structures current state and the entire system would potentially be powered through power-harvesting techniques. Researchers at Virginia Tech have successfully built prototypes of these types of systems and have improved upon them through advances in hardware systems with onboard computing, including actuation and sensing, computational algorithms, and wireless telemetry (Grisso and Inman 2006; Grisso 2005a; b; Inman and Grisso 2006; Kim et al. 2007). Other researchers have used a commercially available impedance chip made by Analog Devices as part of similar systems (Mascarenas 2006; Park et al. 2007a; Park et al. 2007b; Park et al. 2006b). This impedance chip was attempted for use in the work presented in Chapter 4.

\subsection{Thesis objectives and overview}

The need for SHM in wind turbines has been recognized for some time: “...there is much interest in the renewable energy community to develop reliable and quick health monitoring systems for wind turbines" (Simmermacher et al. 1997). Also, the blades of a 
WT are some of the most important components to improve wind power technology and consequently previous researchers have proposed the development of "intelligent" or "smart" blades with on-board SHM capability (Sundaresan et al. 2001). Additionally, this technology could potentially help make wind power more economical by extending blade life and allowing blade designs to be less conservative which would help the wind power industry meet its goals for continued growth in the near future.

The impedance method has been shown to be effective in detecting damage in composites and as an autonomous SHM method, therefore it is anticipated that it would be a promising method for use in an "intelligent" blade. In this work, the feasibility of the impedance method for WTB is investigated. The goal was to see if the impedance method is able to detect damage in this structure and to determine the limits of sensing. This was accomplished by conducting tests on a section of an actual WTB utilizing the method to detect both indirect and actual forms of damage. This research is presented in Chapter 2 and a large portion of this work in contained in Pitchford et al. (2007). In Chapter 3, high-frequency response functions (HFRF) were used on the same WTB section for testing indirect and actual forms of damage. This is an alternative SHM method and is presented for comparison to the impedance method. Based on the results of Chapter 2, the impedance method was used during the fatigue test of a WTB and this work is presented in Chapter 4. Finally, in Chapter 5 a brief summary of this work is presented along with conclusions based on the results. Also, recommendations for future work are made. 


\section{CHAPTER 2 Impedance-Based Structural Health Monitoring of CX-100 Wind Turbine Blade Section}

\subsection{Introduction}

To evaluate IBSHM on WTBs, an actual section of a WTB was obtained from SNL's Wind Energy Technology Department. A series of experiments utilizing piezoceramic (PZT) patches attached to the blade section as self-sensing actuators to detect both indirect and direct forms of damage were performed to test the impedance method. The purpose of these experiments was to validate that the method could detect damage on the blade and to determine the limits of sensing. A large portion of this chapter was presented at the 2007 SPIE Smart Structures and Materials and Nondestructive Evaluation and Health Monitoring conference (Pitchford et al. 2007).

The WTB section tested is from a CX-100 (Carbon eXperimental-100 kW) blade. The CX-100 is a 9 meter long subscale experimental blade that was manufactured by TPI Composites in Rhode Island and was developed by SNL as part of research to incorporate carbon fiber in WTBs. This research included building subscale blades that incorporate carbon fiber which would be tested to static failure, modal tested, fatigue tested, and field tested on actual $100 \mathrm{~kW}$ WTs. The results of these tests were then compared to modeling (Paquette et al. 2007a; Paquette et al. 2006). The section used for this testing comes from a blade that was static tested to failure at NREL's NWTC. The CX-100 has carbon fiber in the spar cap region of the blade infused with the blade skin, which is made of more typical glass fiber and balsa. This can be seen in Figure 2.1 and an overall picture of a similar blade, TX-100, can be seen in Figure 2.2. 


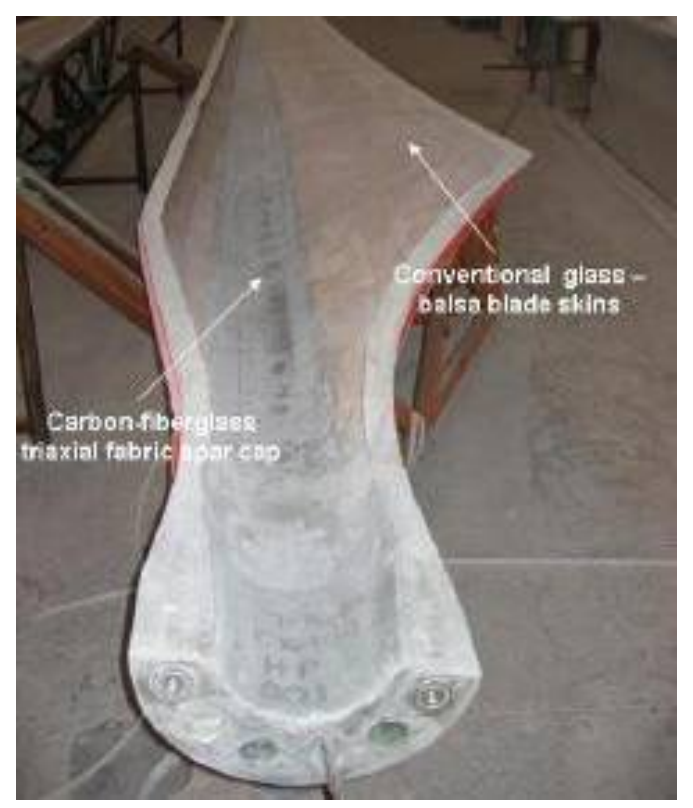

Figure 2.1: CX-100 high pressure skin showing carbon fiber spar cap infused with typical glass/balsa skin. (Paquette et al. 2006)

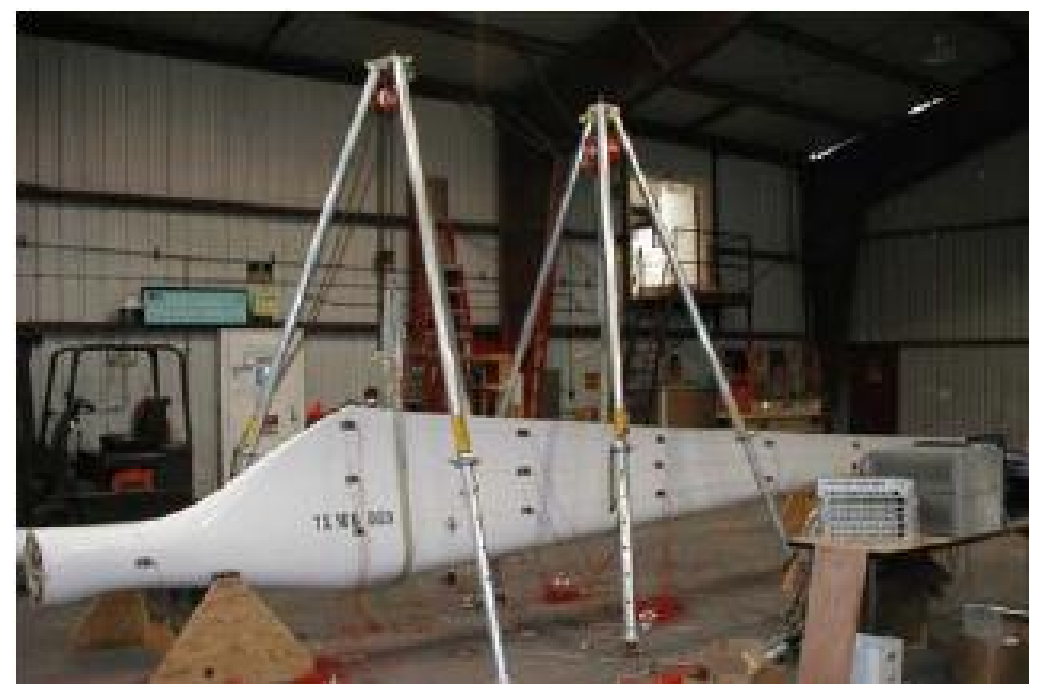

Figure 2.2: Overall picture of TX-100 blade. (Paquette et al. 2006)

The blade section from a CX-100 used for impedance testing is shown in Figure 2.3. In the figure the low pressure (LP) side of the blade is facing upwards and the high pressure (HP) side of the blade is facing downwards. Also in the figure, the crosssectional geometry of the blade, as well as the materials used can be seen. The blade is made up of HP and LP skins and an internal spar which runs the majority of the length of the blade. A protective gelcoat covers the outside of the blade. The leading edge region of the skins is made up of layers of fiberglass, while the trailing edge regions as well as 
the spar are made of balsa covered by fiberglass. Carbon fiber is incorporated into the spar cap regions of the skins. The section used for testing is from just over two-thirds of the way down the length of the blade and is around $40 \mathrm{~cm}$ long.

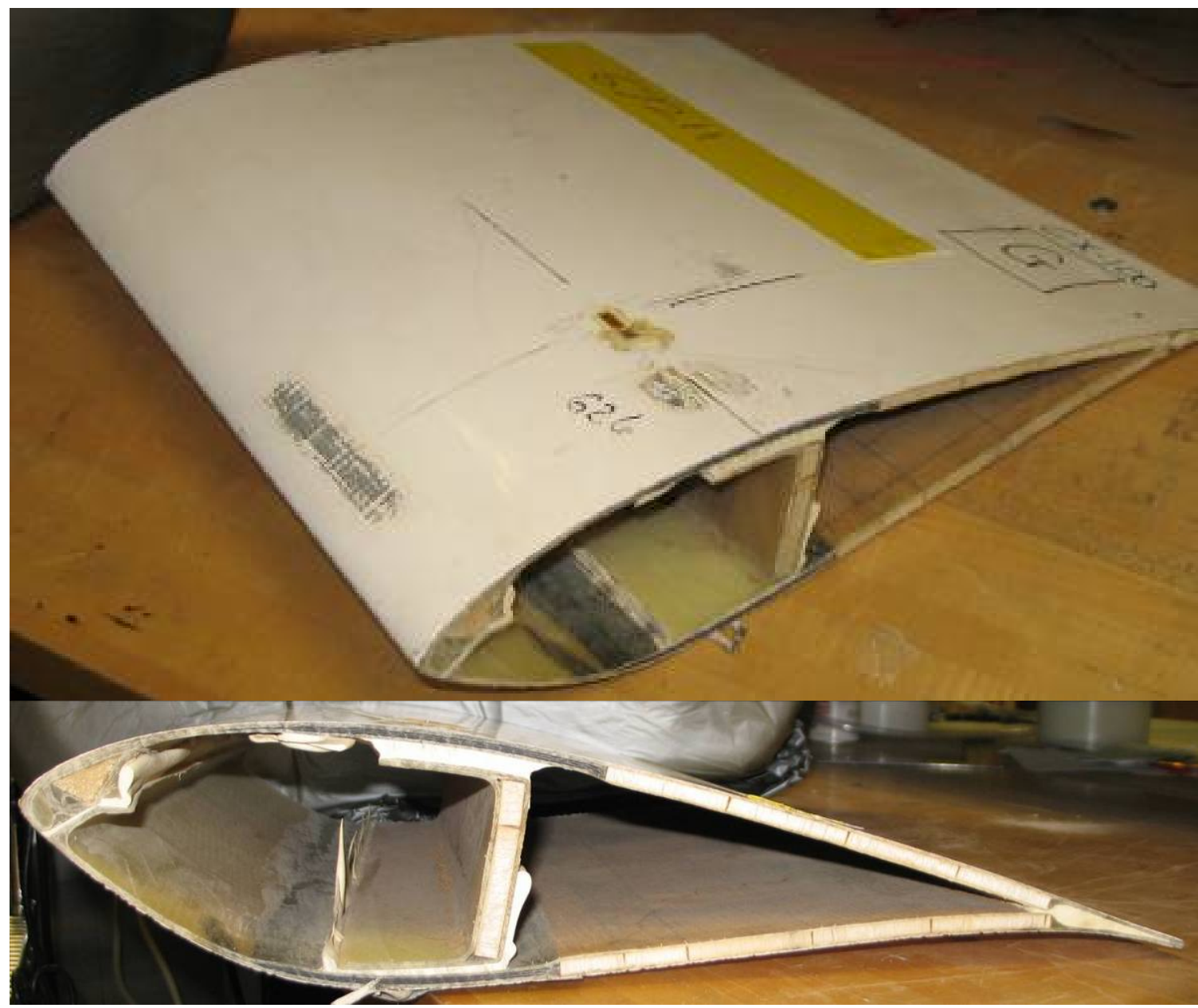

Figure 2.3: CX-100 blade section

Typical damage locations and modes were suggested by SNL based on experience, blade models, and physical testing of the blade. Damage mechanisms are shown in Figure 2.4 on a cross section of the CX-100. Location 1 is on the LP side of the blade, which is where the carbon fiber spar cap meets the balsa skin. The damage mode here is separation of the two sections. Location 2 is adhesive between the spar and the spar cap on the HP side of the blade. The damage mode is cracking of the adhesive. Location 3 is the adhesive between the spar and the spar cap on the LP side of the blade. The damage mode here is pitting of the adhesive. 


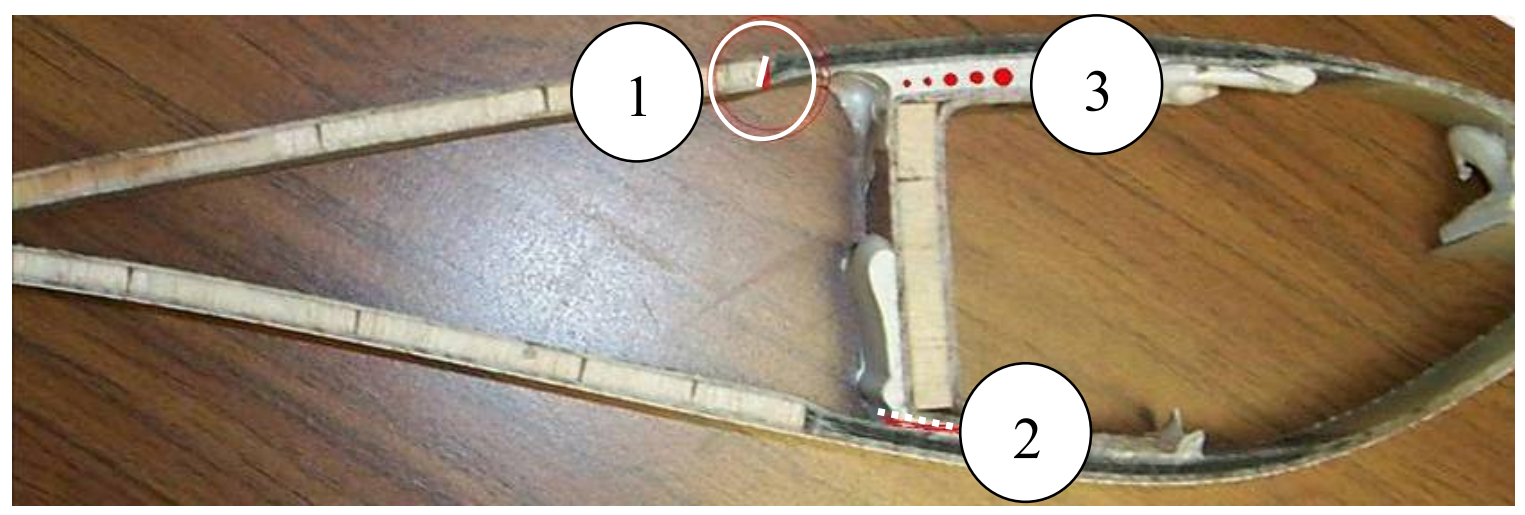

Figure 2.4: CX-100 Cross-section with damage modes

The damage locations suggested by SNL dictated where the indirect and direct forms of damage were simulated. For the indirect testing, damage was simulated in these areas by changing the local mass and stiffness by attaching magnets and clamps to the blade. These types of test were performed because they do not directly damage the blade and are repeatable. This allowed the method to be validated on the blade while getting an idea of proper placement and frequency ranges of sensors before beginning tests that actually physically damage the blade. For the direct damage testing, actual physical damage was simulated in the locations.

\subsection{Experimental setup}

The PZT patches used for IBSHM testing are roughly 2 × $2 \mathrm{~cm}$ and are made from $0.27 \mathrm{~mm}$ thick PSI-5H4E material from Piezo Systems, Inc. They were tested in three locations on the blade which are shown in Figure 2.5. The left patch location is above the balsa skin section near the balsa/carbon intersection and will be referred to as the Skin patch. The patch in the middle is on the fiber glass spar flange and will be referred to as the Spar patch. Finally, the patch on the right is on the carbon fiber spar cap will be referred to as Carbon. To install the Carbon patch some of the extraneous adhesive on the spar cap had to be removed to make room for this sensor as seen in Figure 2.6. Some of the testing was completed before the carbon patch was installed, but these baseline measurements were not compared to those afterwards because of the mass difference. 


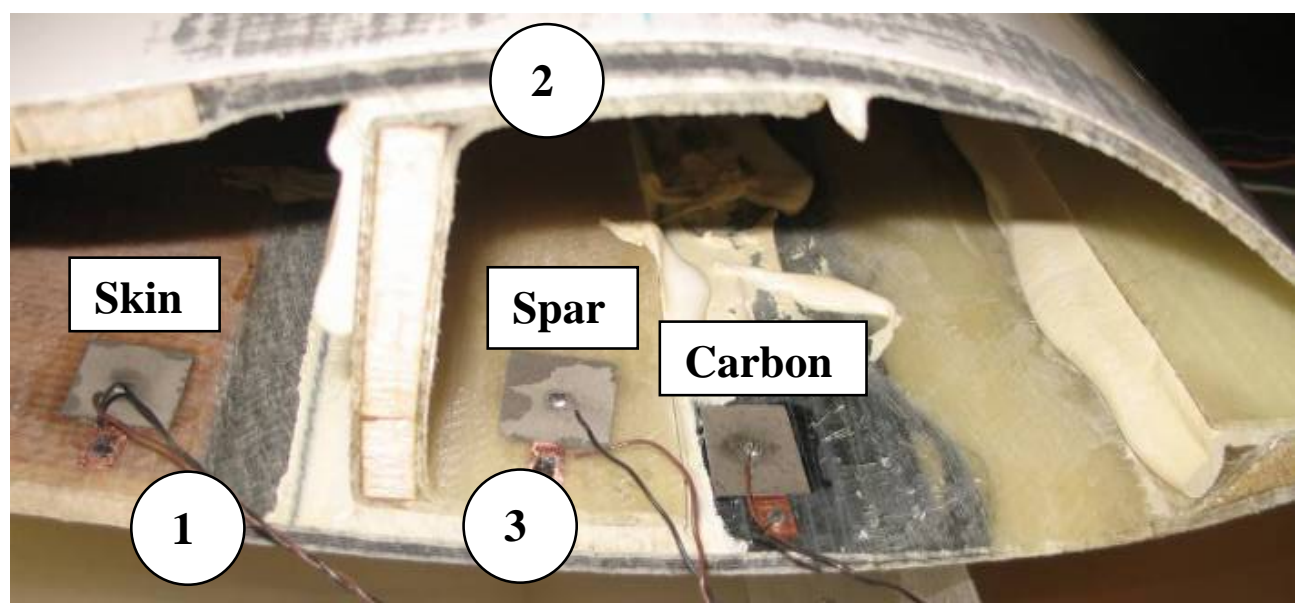

Figure 2.5: Sensor names and locations (damage locations shown for reference)

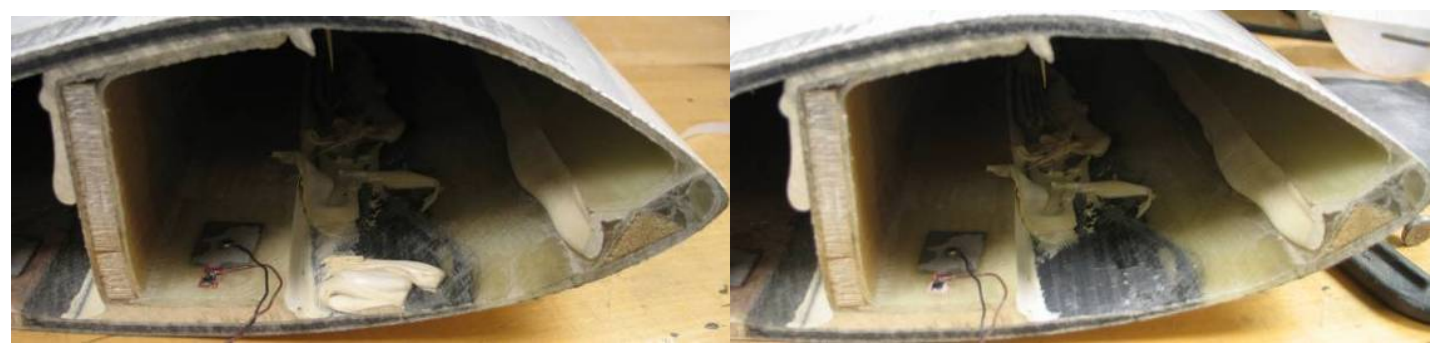

Figure 2.6: Removed adhesive for carbon fiber pzt.

Although the impedance method is typically not affected by boundary conditions, the blade is suspended in nearly free-free condition for consistency for testing as shown in Figure 2.7. The device used for measuring the impedance of the PZTs was an HP4194A impedance analyzer which interfaced with a laptop through a GPIB port. A Labview program developed by Simmers (2005) was used to control the analyzer and record the impedance data. A resolution of $10 \mathrm{~Hz}$ was used in gathering impedance data with a 1 volt magnitude excitation and settings on the HP4194A of 4 averages and "medium" integration time. 


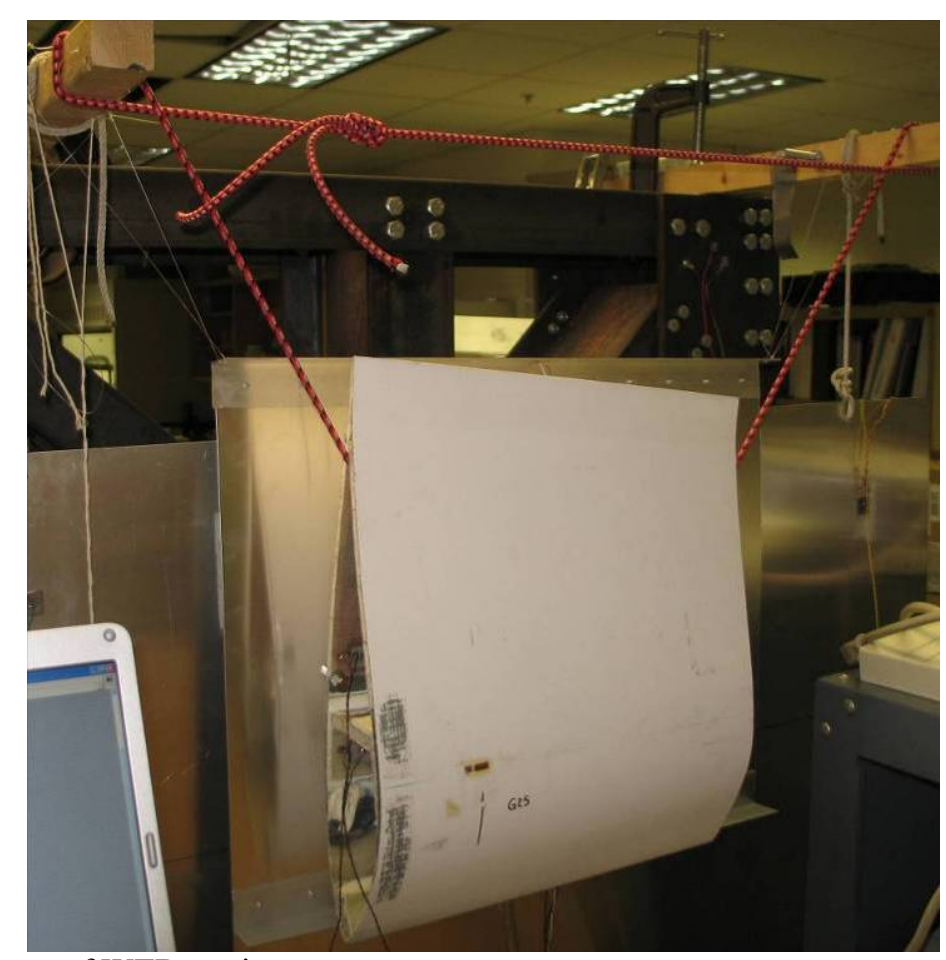

Figure 2.7: Test setup of WTB section.

\subsubsection{Procedure}

The indirect and direct damage IBSHM tests that follow consist of first obtaining multiple baseline impedance signatures of the blade in an undamaged state. Then the desired damage is added, and the damaged impedance curve is obtained. To compare the impedance signatures of the multiple undamaged and damaged structure states, a scalar damage metric was used, as discussed in section 1.4. The damage metric used in this work is the root mean squared deviation (RMSD) metric:

$$
M=\sqrt{\sum_{i=1}^{n} \frac{\left(Z_{1, i}-\left(Z_{2, i}-\delta\right)\right)^{2}}{Z_{1, i}^{2}}}
$$

where $Z_{1}$ is the real part of the baseline impedance, and $Z_{2}$ is the real part of the impedance in question. $n$ is the total number of data points, and $i$ is each individual data point. $\delta$ is the difference in the average values of $Z_{1}$ and $Z_{2}$. This metric is scaled by the 
baseline measurement and is corrected for vertical shift between measurements by subtracting $\delta$ from the interrogation impedance. This vertical shift does not indicated damage but rather is due to changing environmental conditions such as temperature.

For each test, a damage threshold is determined based on the baseline metrics to characterize whether or not a metric from damaged impedance signatures is sensing damage. For an actual blade application this would likely be determined experimentally, but for these laboratory tests which are a starting point for IBSHM on WTBs, a statistical approach is used based on the variation of the baseline metrics. The statistical hypothesis test which dictates finding the threshold is a pooled T-test which compares two unknown population means which have approximately equal variation. In the case at hand, the unknown means are the undamaged and damaged metrics. Their variation, which is due to measurement error and changing laboratory conditions, would be approximately equal for undamaged and damaged cases. For this hypothesis test, the null hypothesis is that the undamaged and damaged metrics are equal while the alternative hypothesis is that the damaged metric is greater than the undamaged metric. The threshold is the smallest value of the damaged metric which makes the alternative hypothesis true which is

$$
T H=\bar{y}_{\text {base }}+s_{\text {base }} t \sqrt{\frac{1}{n_{\text {base }}}+1}
$$

where $\bar{y}_{\text {base }}$ is the average value of the undamaged metrics, $s_{\text {base }}$ is the standard deviation of the undamaged metrics, $n_{\text {base }}$ is the number of samples of the undamaged metrics, and $\mathrm{t}$ is the value of the t-distribution for $n_{\text {base }}-1$ degrees of freedom and assuming a $95 \%$ level of confidence. This equation also assumes that there is only one sample being taken for the damaged metric.

So in each test for a given frequency range, a set of baseline measurements are taken on the undamaged structure. These are compared using the RMSD damage metric and then a damage threshold is calculated using the previously described formula. 
Finally, the RMSD is used to compare the damaged impedance signatures to the original baseline. If these damaged metrics are greater than the threshold, they are considered to be sensing damage. For comparison purposes, each set of undamaged and damage metrics are scaled so that the average of the baseline metrics is equal to one. In the results section, the threshold is shown as a horizontal line on the damage metric charts and was found to be close to a value of 2 scaled RMSD for the majority of the indirect and direct damage IBSHM tests.

\subsubsection{Indirect damage testing}

The first type of tests performed on the blade were indirect forms of damage such as adding mass to and changing the local stiffness of the blade. The indirect damage being simulated was based on damage locations provided by SNL. Simulated damage by adding mass was accomplished by attaching magnets to the blade with a combined mass of approximately 25 grams at the three damage locations. The magnets can be seen in Figure 2.8 in location 1 . Also, to simulate damage by changing the local stiffness of the blade in the three locations, a 2 inch C-clamp was attached to the blade, as seen in Figure 2.8 at location 2. The C-clamp also added 215 grams of mass to the blade. Finally, to get an idea of the range of sensing, additional tests were run moving the masses down the blade away from the sensors. Masses were kept in the same three locations relative to the cross section of the blade but were placed at 13,25 , and $40 \mathrm{~cm}$ away from the sensor end of the blade. These distances also roughly correspond to $1 / 3,2 / 3$, and all the way down the blade section.
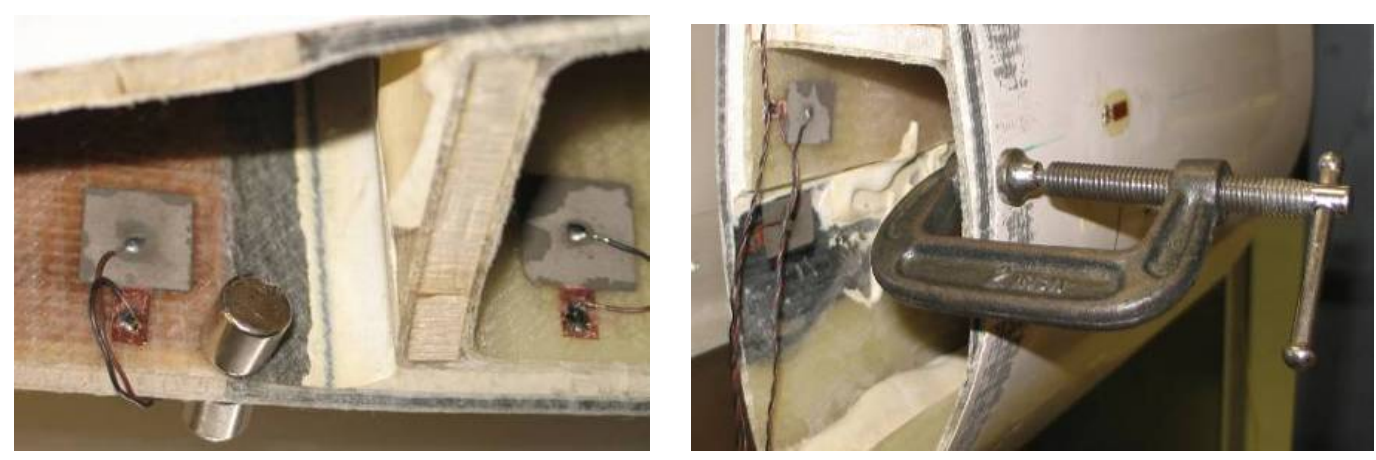

Figure 2.8: Indirect damage methods: Added mass (25g magnets) in location 1 and added stiffness (Cclamp) in location 2 . 
Tests were also run with additional mass in the same locations near the sensor and down the blade. One-hundred gram weights were placed on each magnet to obtain 225 grams of total weight. This configuration can be seen in Figure 2.9. However, the results of these tests are omitted because they produced smaller damage metrics than the 25 gram tests. This was exactly the opposite effect that was desired with a larger mass but was likely due to the fact that adding mass actually made the magnets have a more difficult time staying together so despite adding mass, the clamping force of the magnets on the structure was actually less. This was the case so much in fact that no results were obtained for location 3 because the magnets would not stay clamped to the blade.

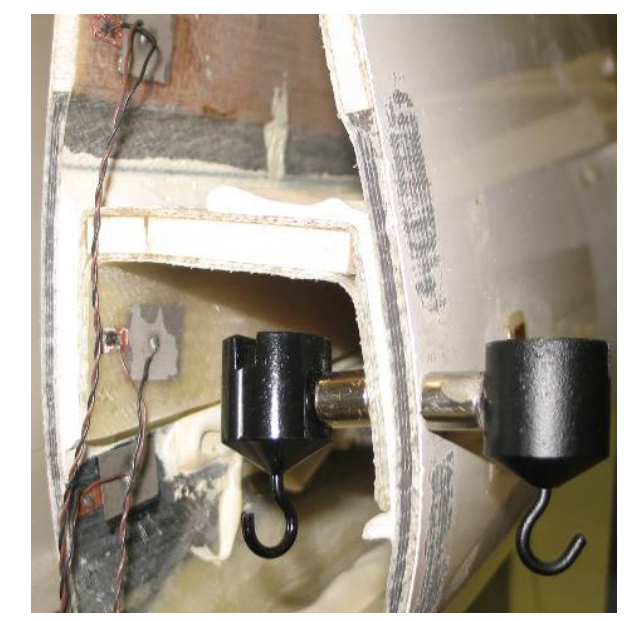

Figure 2.9: Additional (225g) mass simulated damage at location 2

\subsubsection{Actual damage testing}

After the indirect damage testing was complete, direct forms of damage were tested. Locations 1 and 3 were chosen for testing because the damage to these areas was easier to simulate, especially down the length of the blade. In addition, based on the indirect damage testing, the PZTs had relatively small success in sensing damage on the opposite side of the blade, therefore trying to sense damage on the same side for actual damage seemed more logical. Damage was induced at both the sensing end of the blade and down the length of the blade for both locations. 
Location 1 is the connection of the carbon fiber spar cap to the balsa skin. The damage mode at this location is separation of the two sections. Damage at the sensing end was produced by driving a chisel in between the two sections. This damage was added progressively, with three baselines being taken initially and then impedance measurements taken after each progression of damage and compared to the initial baseline. Three damage states were produced: an $8 \mathrm{~mm}$ deep $/ 3 \mathrm{~mm}$ wide at opening notch, a $15 \mathrm{~mm}$ deep $/ 5 \mathrm{~mm}$ wide at opening notch, and a $19 \mathrm{~mm}$ deep $/ 5 \mathrm{~mm}$ wide at opening notch. This damage can be seen in Figure 2.10 moving left to right. Damage in this location was also tested down the length of the blade in the form of $2.5 \mathrm{~cm}$ long gaps in the balsa/carbon fiber by driving a chisel into the gap from the outside of the blade, as is seen in Figure 2.11, at 3, 12, 22, and $31 \mathrm{~cm}$ down the blade. The gelcoat on the outside of the blade was removed initially for all locations before the initial baselines so that only the effect of separating the balsa and carbon fiber was taken into account. For these tests, two baselines were initially taken, and two measurements were taken after each damage state (starting from the far end of the blade and working back towards the sensing end). Since the cumulative effect of the damage was not desired in these tests, each set of two measurements was compared to the previous two, and then would become the baseline for the next damage state, and so forth.

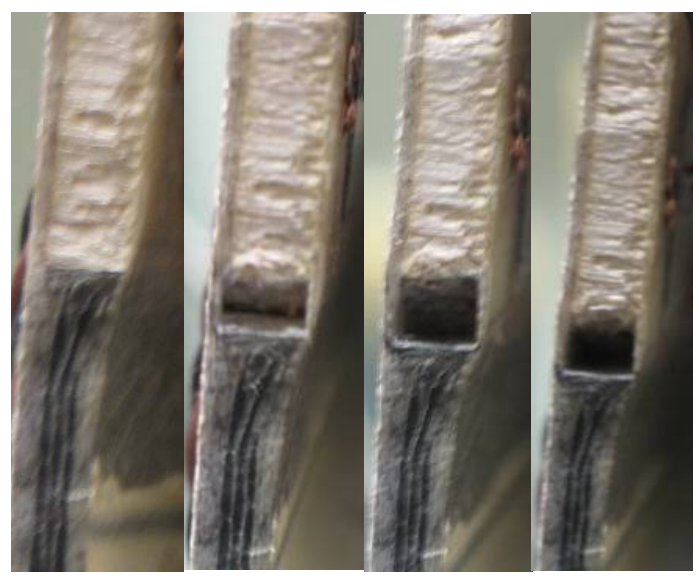

Figure 2.10: Progressive damage in location 1 


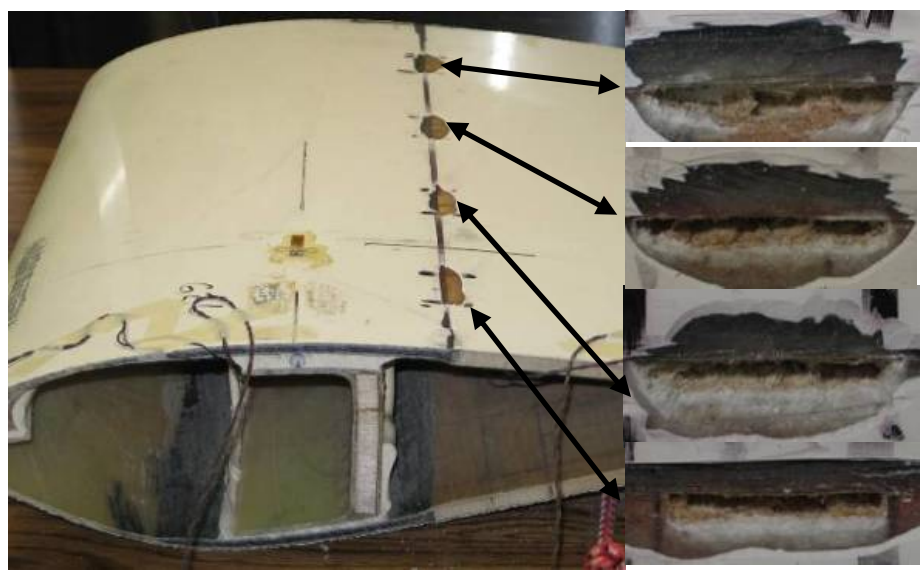

Figure 2.11: Damage in location 1 located down the length of the blade

For location 3, a series of tests similar to those for location 1 were conducted. Location 3 is the adhesive between the spar flange and the spar cap, and the damage mode in this location is pitting of the adhesive. For the progressive damage at the sensor end of the blade, holes of increasing size were drilled into the adhesive $4 \mathrm{~cm}$ deep with diameters: 1.6, 3.2, 4.8, and $6.4 \mathrm{~mm}$. This damage can be seen in Figure 2.12 moving from left to right. In Figure 2.13, the locations down the length of the blade are shown. These holes were drilled as close to perpendicular to the length of the blade as possible, but due to the geometry of the blade section not all the holes are perfectly perpendicular. Slightly different angled holes are not anticipated to be a problem since damage is applied no matter what the angle is. These holes are all roughly 5-6 cm deep, $6.4 \mathrm{~mm}$ in diameter, and were drilled (starting at the far end and moving toward the sensing end) at $0,5,13,20$, and $28 \mathrm{~cm}$ from the sensors.

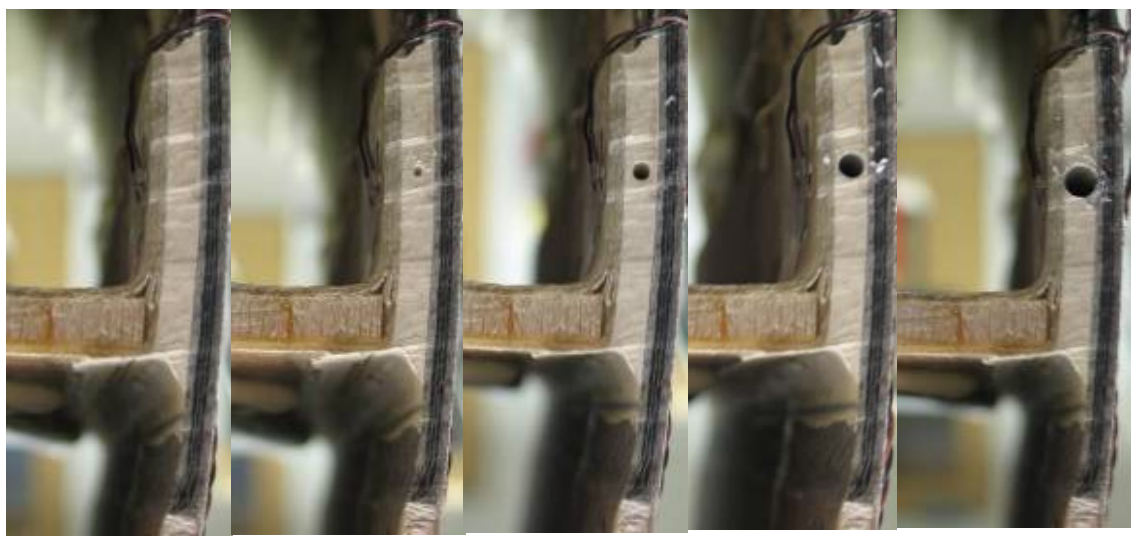

Figure 2.12: Progressive damage in location 3 


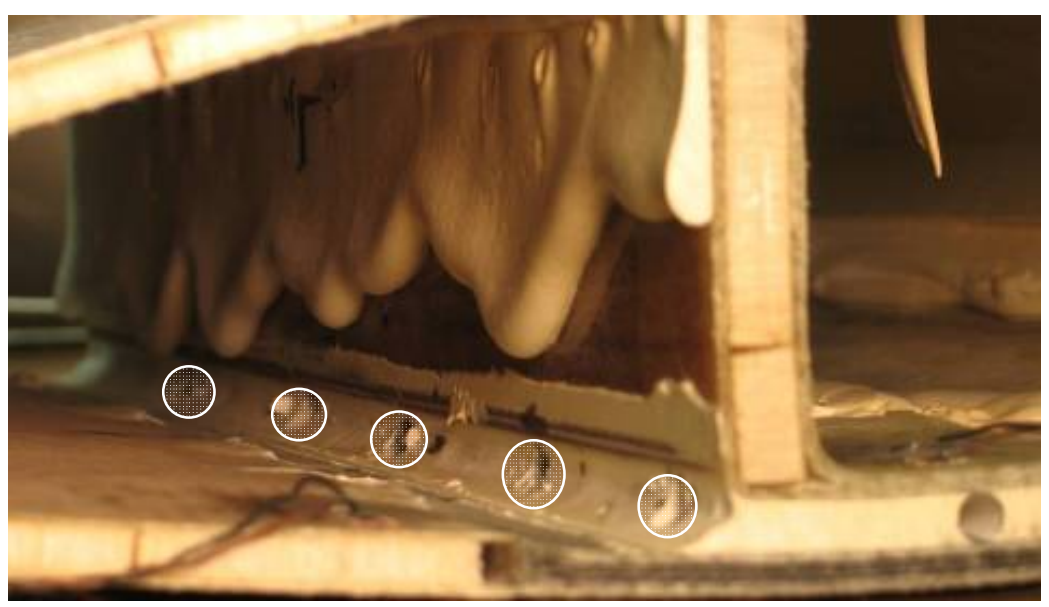

Figure 2.13: Damage in location 3 located down the length of the blade

\subsection{Indirect Damage Results}

Initially, baseline impedance signatures were obtained for all three PZT patches from $1-200 \mathrm{kHz}$ to see the characteristics of the impedance signatures. An example can be seen in Figure 2.14 and the baselines for the other two patches are in Appendix A. The impedance baselines show for all three PZTs for frequencies greater then $60 \mathrm{kHz}$, the impedance has a relatively flat response with peak density lower than typically used for the impedance method. The range from $3-30 \mathrm{kHz}$ has more typical peak density, although some of this range $(3-10 \mathrm{kHz})$ is lower than typical frequencies used for impedance based SHM. Low structural response at higher frequencies is likely due to the high structural damping of the blade, especially that of the balsa. 

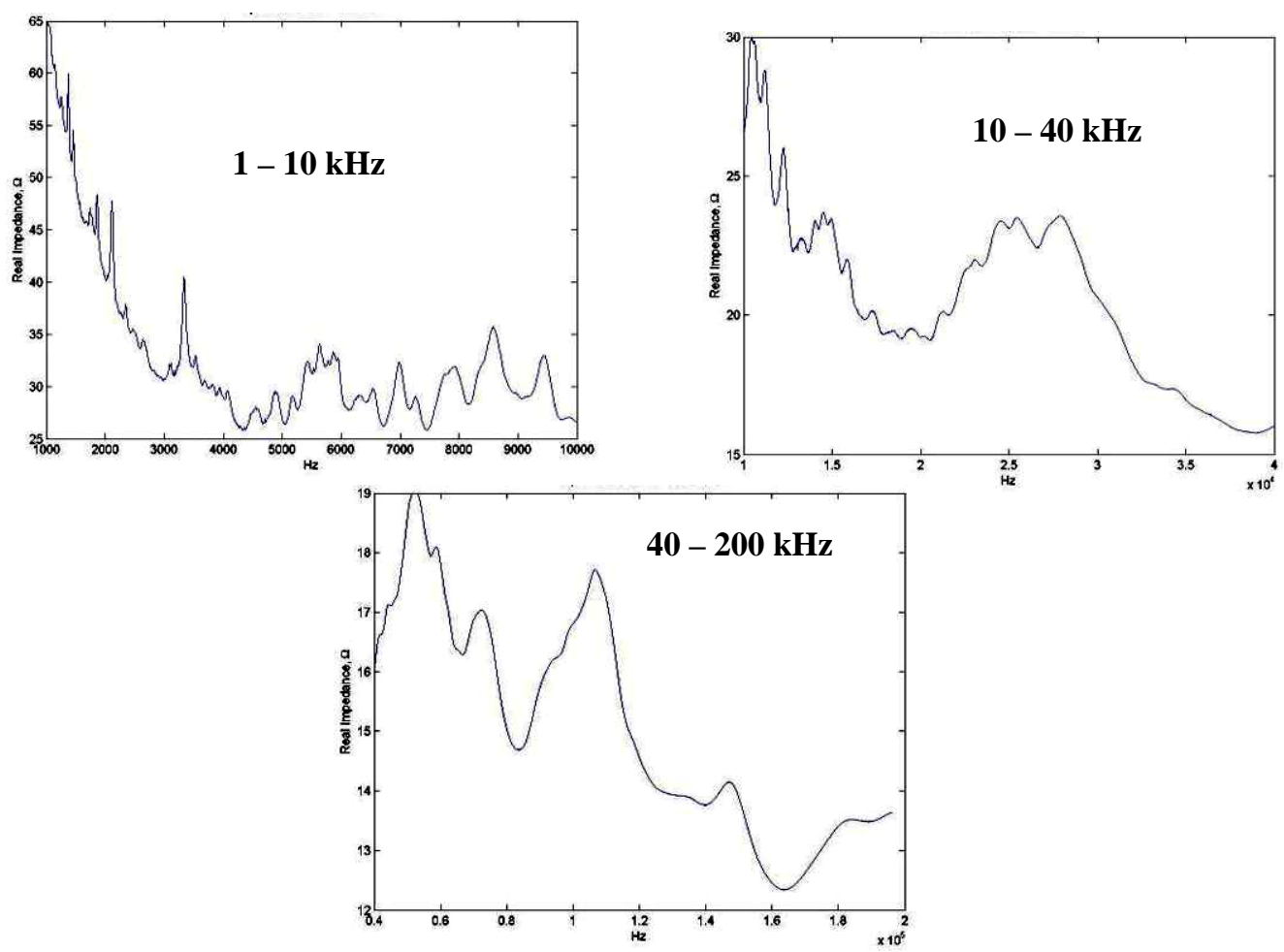

Figure 2.14: Spar baseline impedance

For the magnet and C-clamp tests, several frequency ranges were investigated within the range $3-30 \mathrm{kHz}$ : 3-10, 10-17, 17-24, 24-31, 10-30, and 3-20 kHz. Also several frequency ranges greater than $30 \mathrm{kHz}$ were also investigated for the C-clamp tests: $30-60,60-90,90-110$, and $30-100 \mathrm{kHz}$. Examples of the impedance plots from indirect damage testing are shown in Figure 2.15 and Figure 2.16. They are both from the spar sensor from 10-30 kHz. Figure 2.15 is the added mass test and Figure 2.16 is the added stiffness test for all three locations. Change in the impedance from baseline to damage states can clearly be seen. The remaining impedance signatures from the indirect damage tests are shown in Appendix A. 


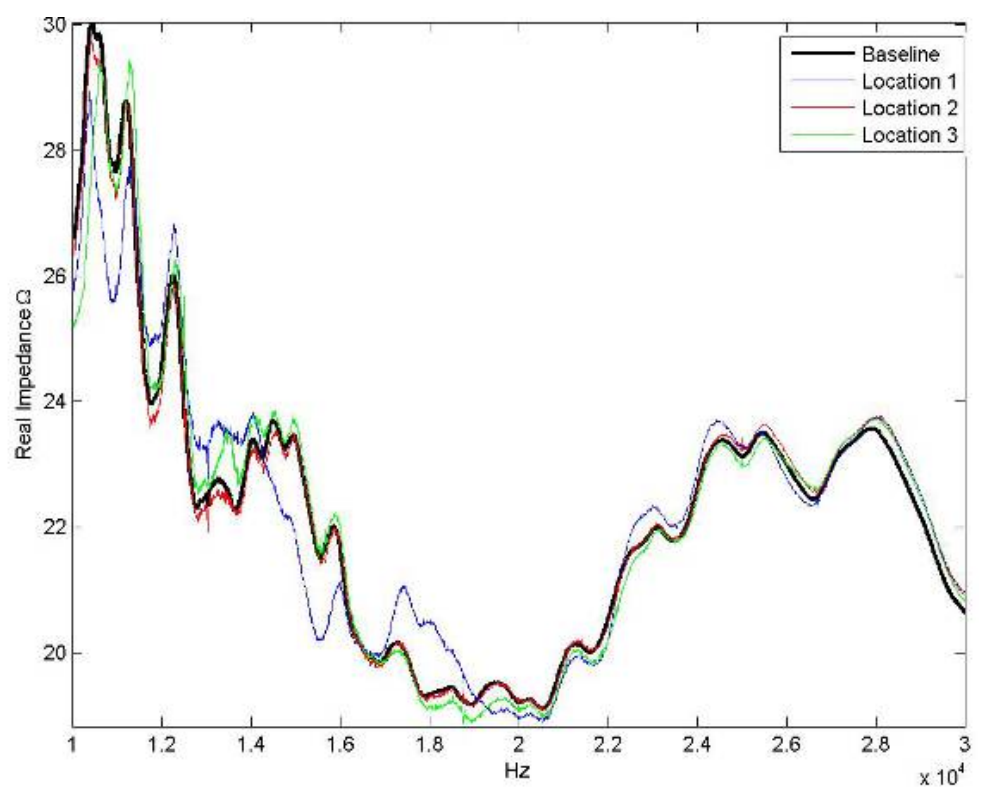

Figure 2.15: Impedance plot for spar sensor, $10-30 \mathrm{kHz}$, added mass

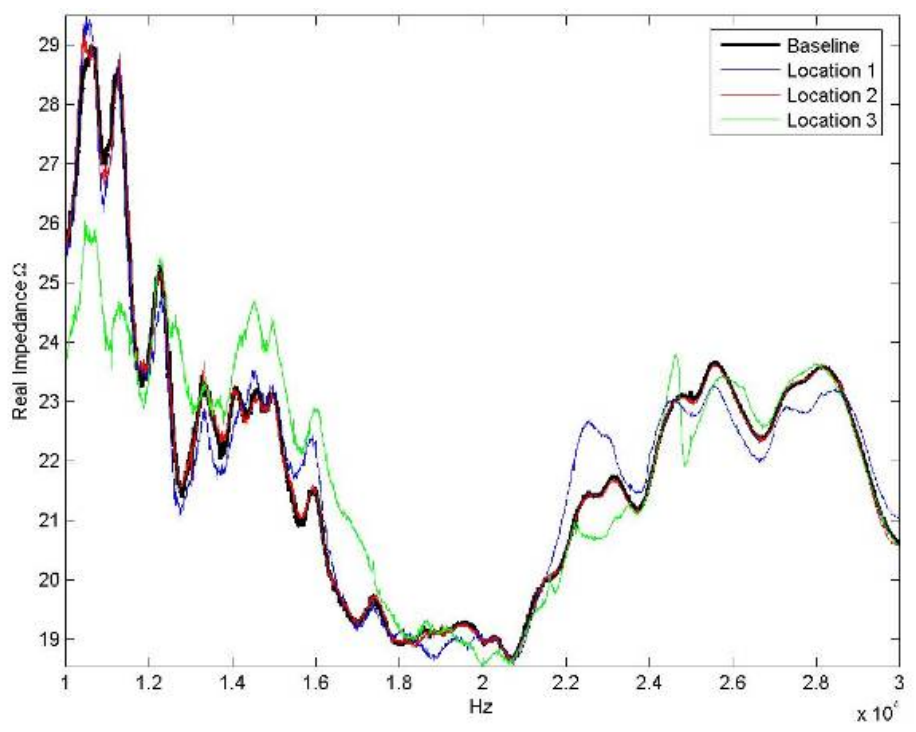

Figure 2.16: Impedance plot for spar sensor, $10-30 \mathrm{kHz}$, added stiffness (C-clamp)

\subsubsection{Added mass results}

This section contains the results for the simulated damage using the 25 gram magnets at the sensor end of the blade at locations 1, 2, and 3 for the three sensors. The results are shown in Figure 2.17 - Figure 2.19 . Again, in damage metric charts, each group of damage metrics is scaled so that the average of baseline metrics is equal to one. Also, the undamaged metric represents the average of multiple measurements. 


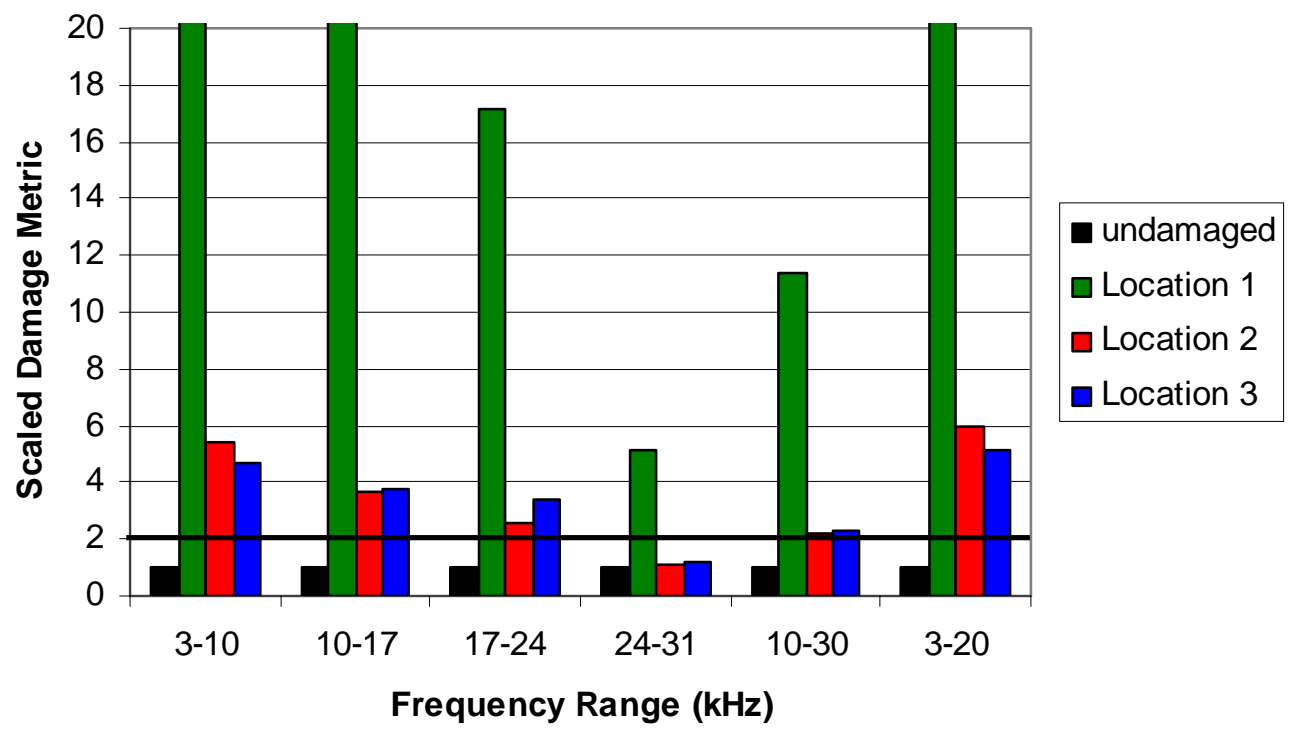

Figure 2.17: Skin: Added mass (25g): Damage metrics calculated for several frequency ranges.

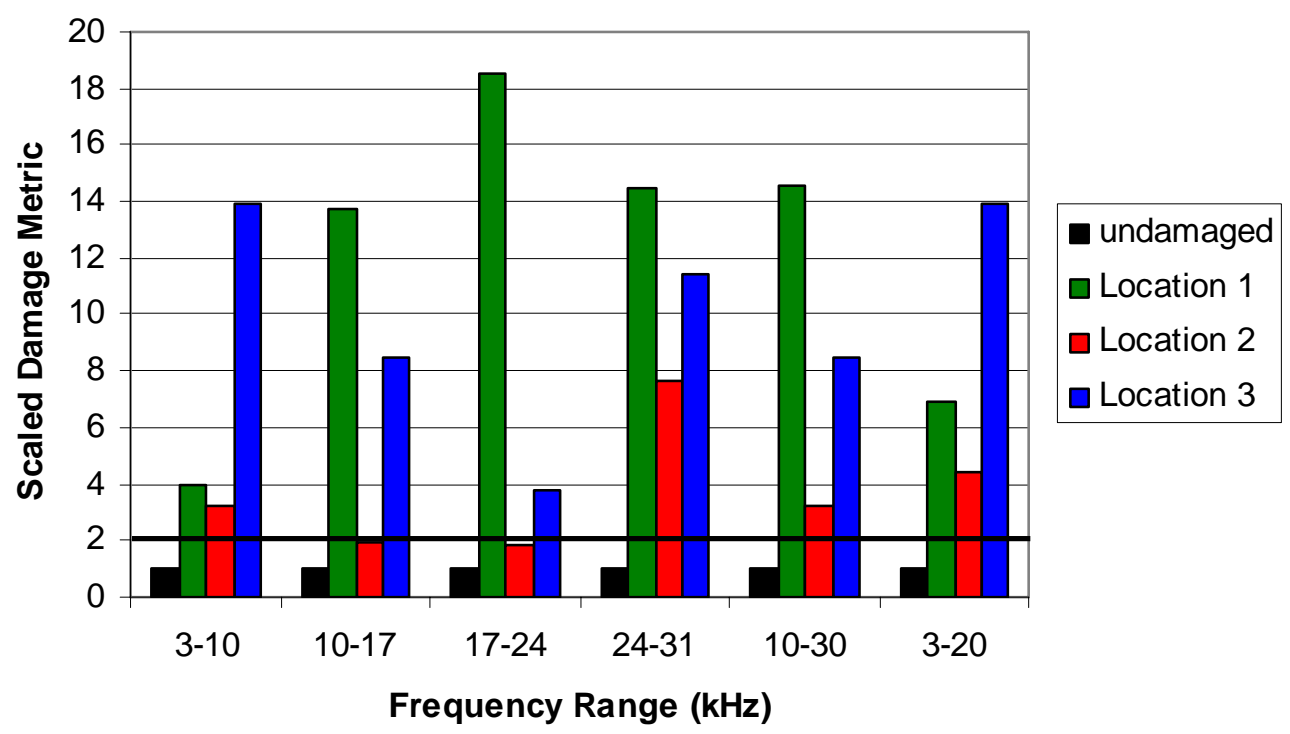

Figure 2.18: Spar: Added mass ( $25 \mathrm{~g})$ : Damage metrics calculated for several frequency ranges. 


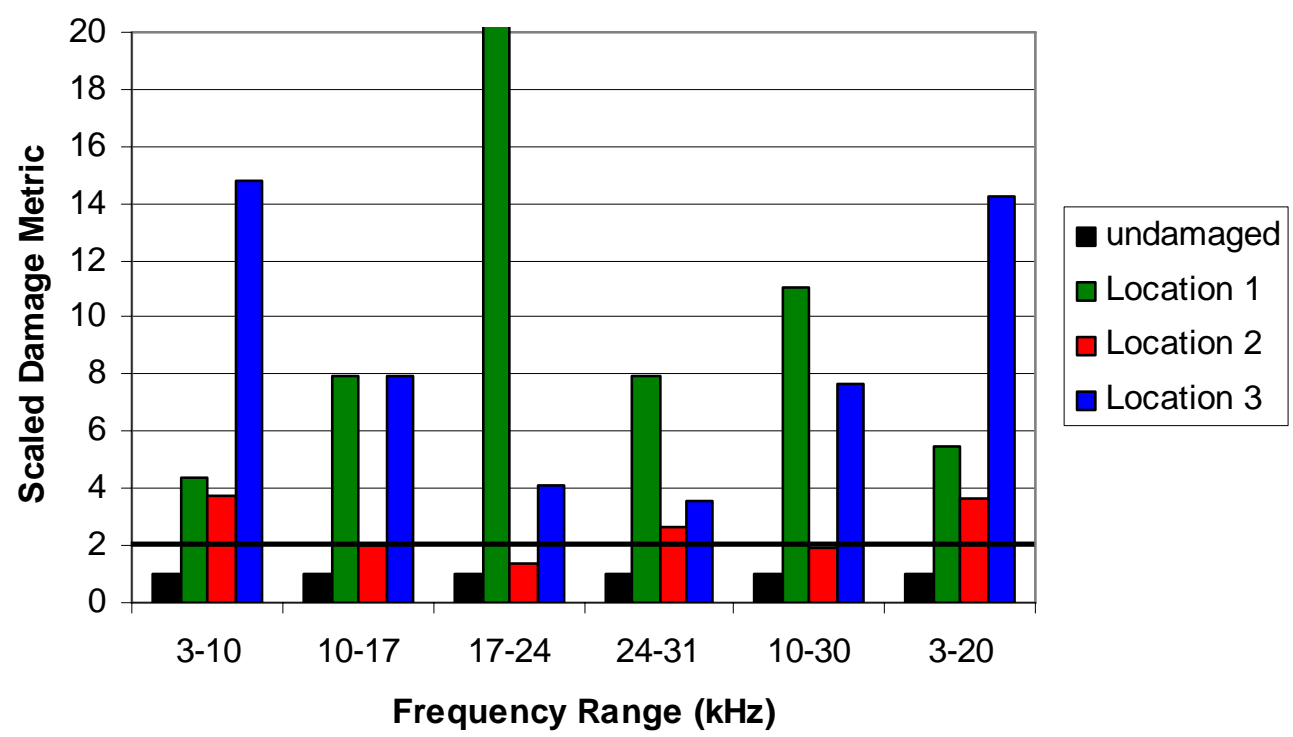

Figure 2.19: Carbon: Added mass (25 g): Damage metrics calculated for several frequency ranges.

From the results, all three sensors are able to sense damage in nearly all of the frequency ranges for the three damage locations. However, looking at the impedance plots in Appendix A shows that the 10-17, 17-24, and 24-31 kHz ranges have rather low peak densities, despite showing damage in the damage metric plots. For example, the spar sensor in the $24-31 \mathrm{kHz}$ range has relatively high damage metrics, as seen in Figure 2.18, but looking at Appendix A at the corresponding impedance plot this range only contains three peaks. The damage metrics for this case and the other low peak density ranges are questionable.

The other ranges: 3-10, 10-30, and 3-20 kHz have more acceptable peak densities. The $10-30 \mathrm{kHz}$ range is the most appealing due to the fact that this frequency range is the highest and the most typical of previous impedance-based SHM (greater than $10 \mathrm{kHz}$ ). As can been seen in Figure 2.17, the top or skin sensor most effectively senses damage at location 1 which is located directly beside it. It doesn't effectively sense location 2 or 3 in the $10-30 \mathrm{kHz}$ range although it does in the $3-10$ and $3-20 \mathrm{kHz}$ ranges. This is probably due to the more global nature of the lower frequencies contained in these ranges, which sense damage but would be sensitive to boundary condition changes. 
The carbon sensor in Figure 2.19 is able to sense location 1 and 3 quite well. It is able to sense location 2, which is on the other side of the blade, but similar to the skin PZT location, only in the more global low frequency ranges. The spar sensor in Figure 2.18 is able to sense all three locations quite well. It is able to sense location 2 on the other side of the blade, even in the $10-30 \mathrm{kHz}$ range. This is the only sensor that is able to sense location 2 on the opposite side of the blade in the $10-30 \mathrm{kHz}$ range.

From these results it is clear that the PZT patches sense damage better when the path between the sensor and the damage location does not contain balsa. This makes the skin sensor rather ineffective except for the damage directly beside it or with low frequencies where the sensing is more global. The spar and carbon sensor locations were much more effective in sensing both locations 1 and 2, while the spar sensor was the only sensor that was able to sense location 2 in the higher frequency range.

\subsubsection{Added stiffness results}

This section contains the results for the simulated damage using the C-clamp at the sensor end of the blade at locations 1, 2, and 3 for the three sensors. The results are shown in Figure 2.20 - Figure 2.22.

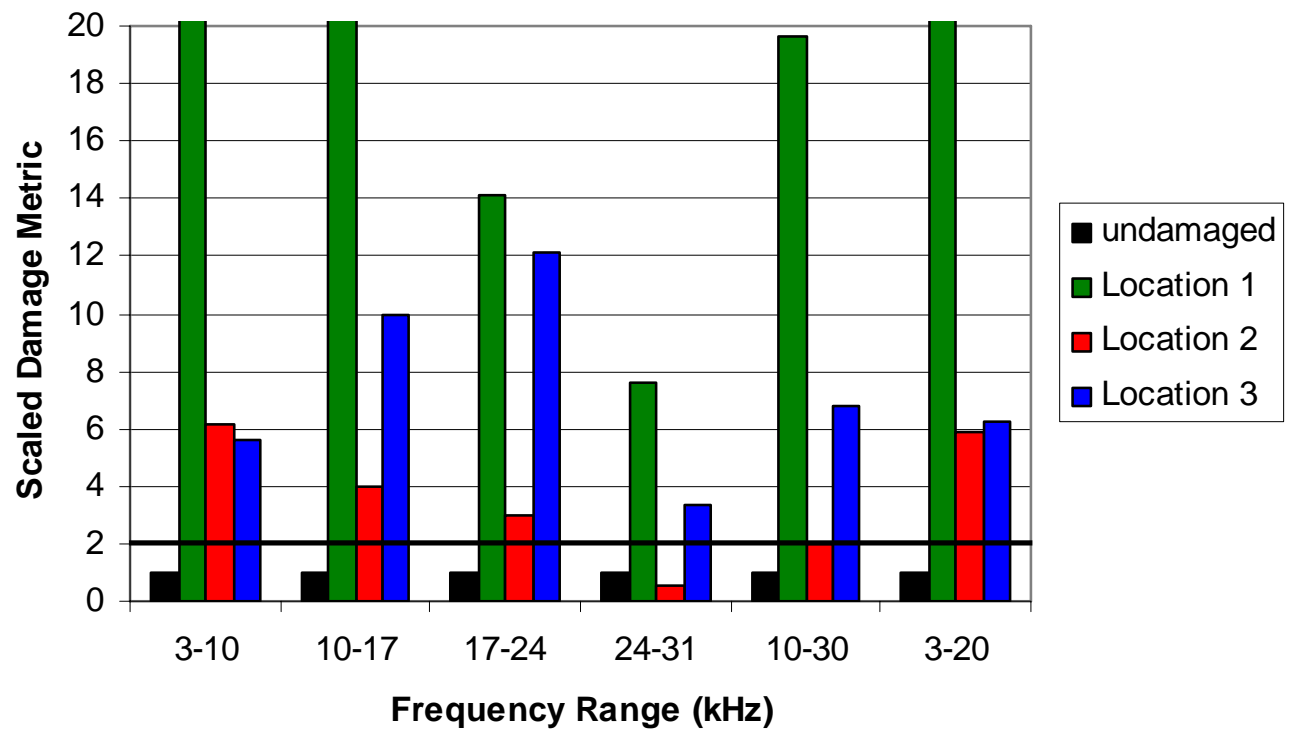

Figure 2.20: Skin, clamp: Damage metrics calculated for several frequency ranges. 


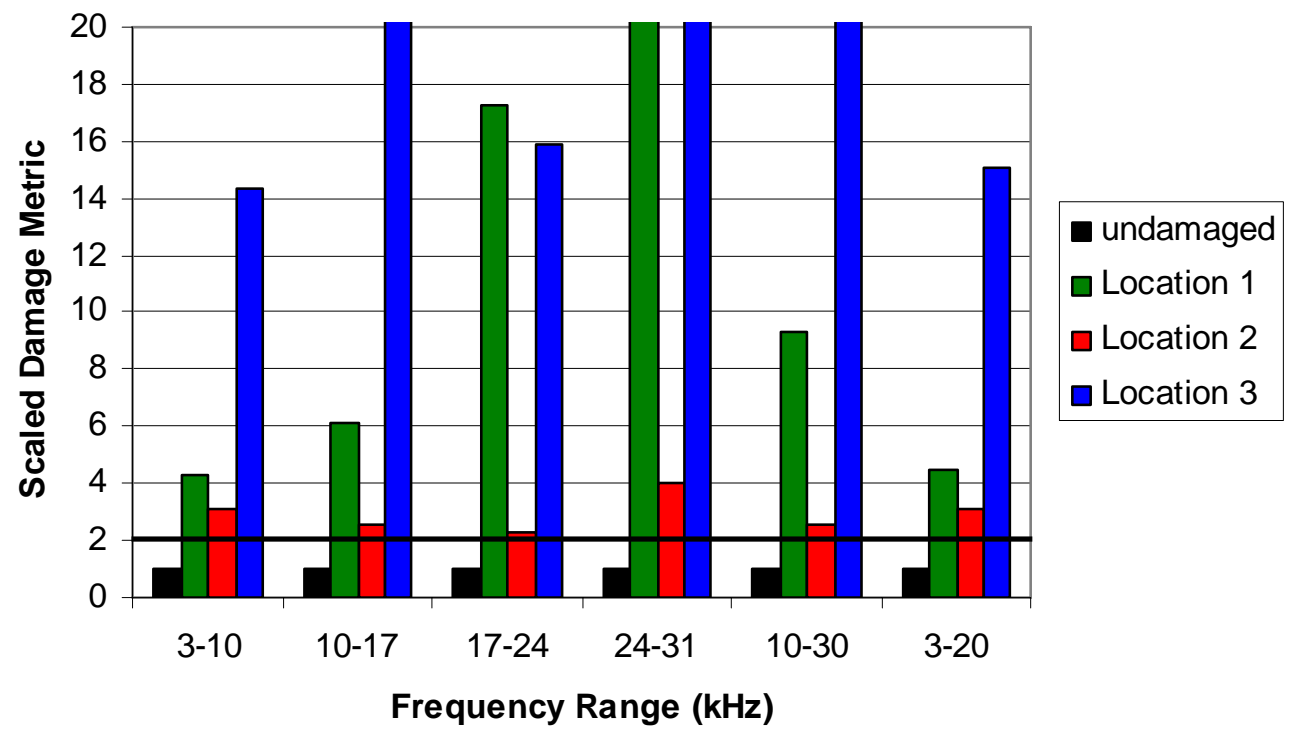

Figure 2.21: Spar, clamp: Damage metrics calculated for several frequency ranges.

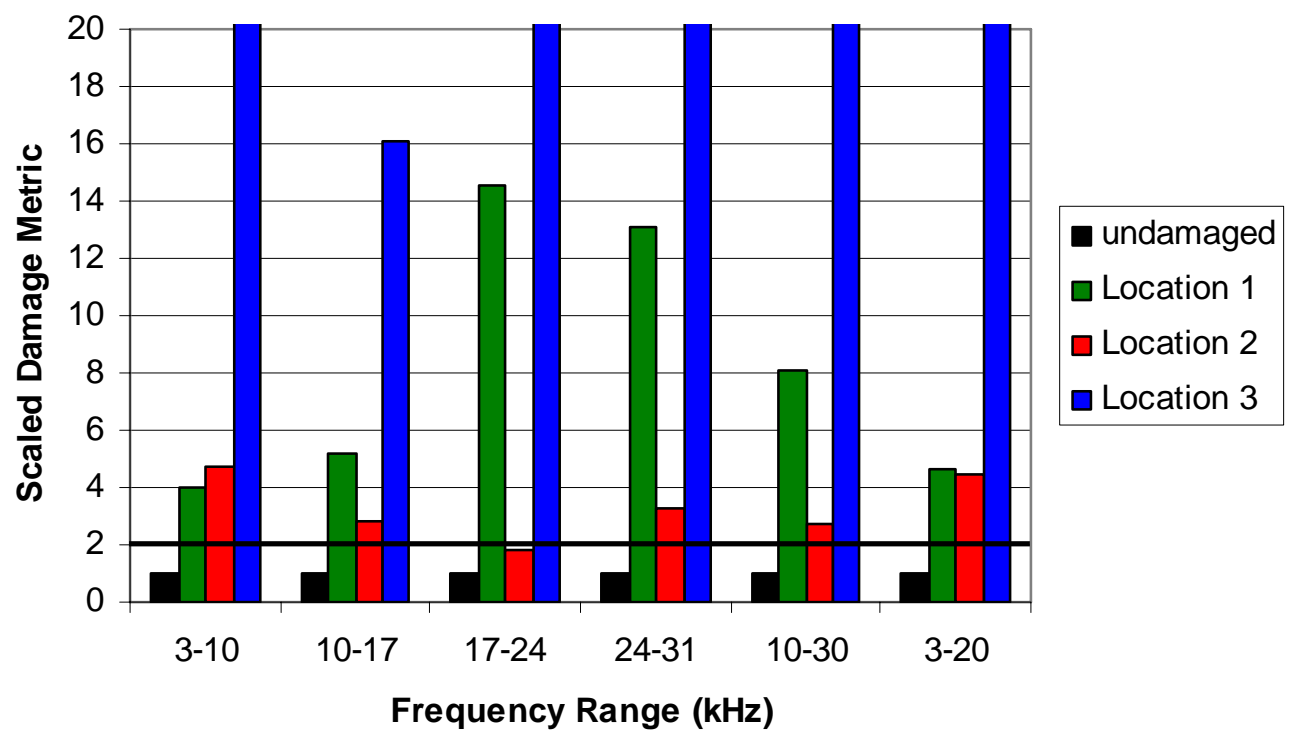

Figure 2.22: Carbon, clamp: Damage metrics calculated for several frequency ranges.

The results of the simulated damage with C-clamp tests are quite similar to the added mass tests. For locations 1 and 3 the trends for this test follow that of the added mass test but in general the damage metrics are higher, which is consistent with the clamp 
being a higher degree of simulated damage (more mass, and local stress to the area). This is somewhat true for location 2 but not to the same degree. All sensors have a more difficult time sensing location 2 , which is on the opposite side of the blade.

\subsubsection{Added stiffness, higher frequency results}

This section contains the results for the simulated damage using the C-clamp at the sensor end of the blade at locations 1, 2, and 3 for the three sensors. With the Cclamp damage more change was seen in impedance plots in the higher frequency range (30-100 kHz) more typical of impedance-based SHM. Several frequency ranges were investigated in this higher range: $30-60,60-90,90-110$, and 30-100 kHz. The damage metric results are shown in Figure 2.23 - Figure 2.25.

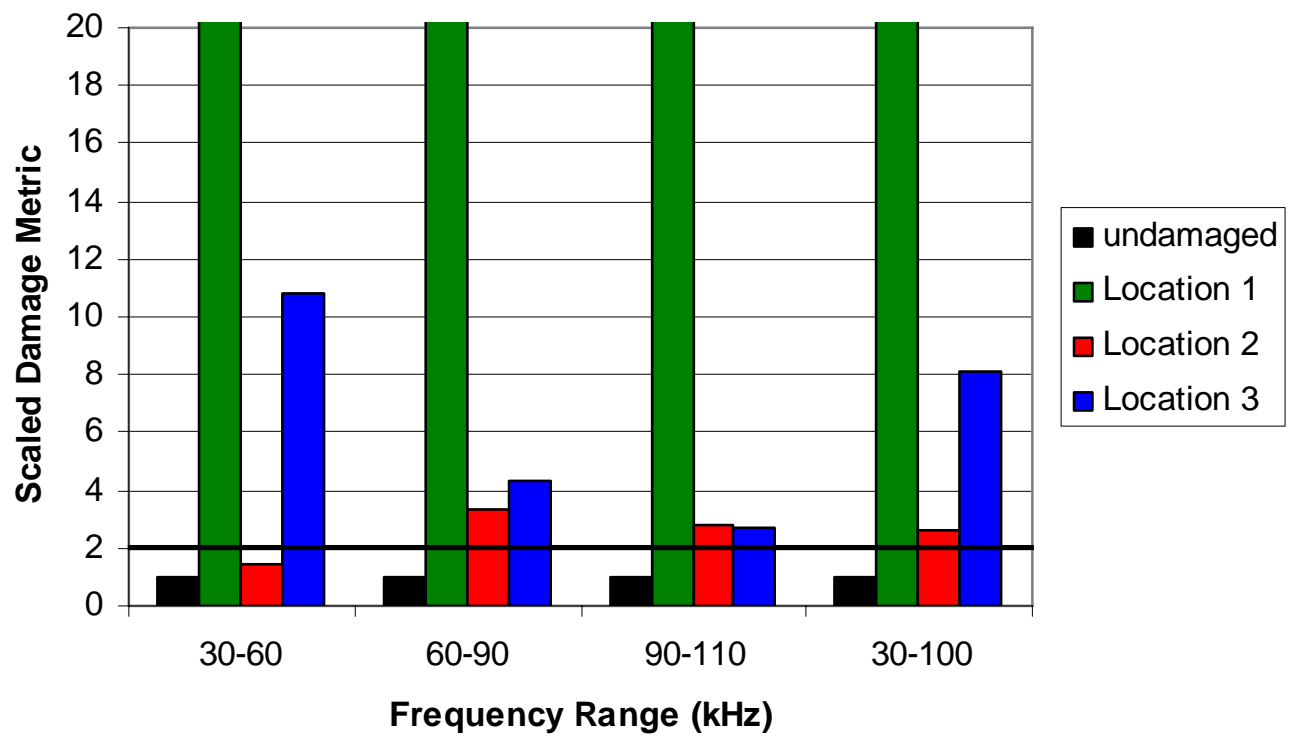

Figure 2.23: Skin, clamp: Damage metrics calculated for several frequency ranges. 


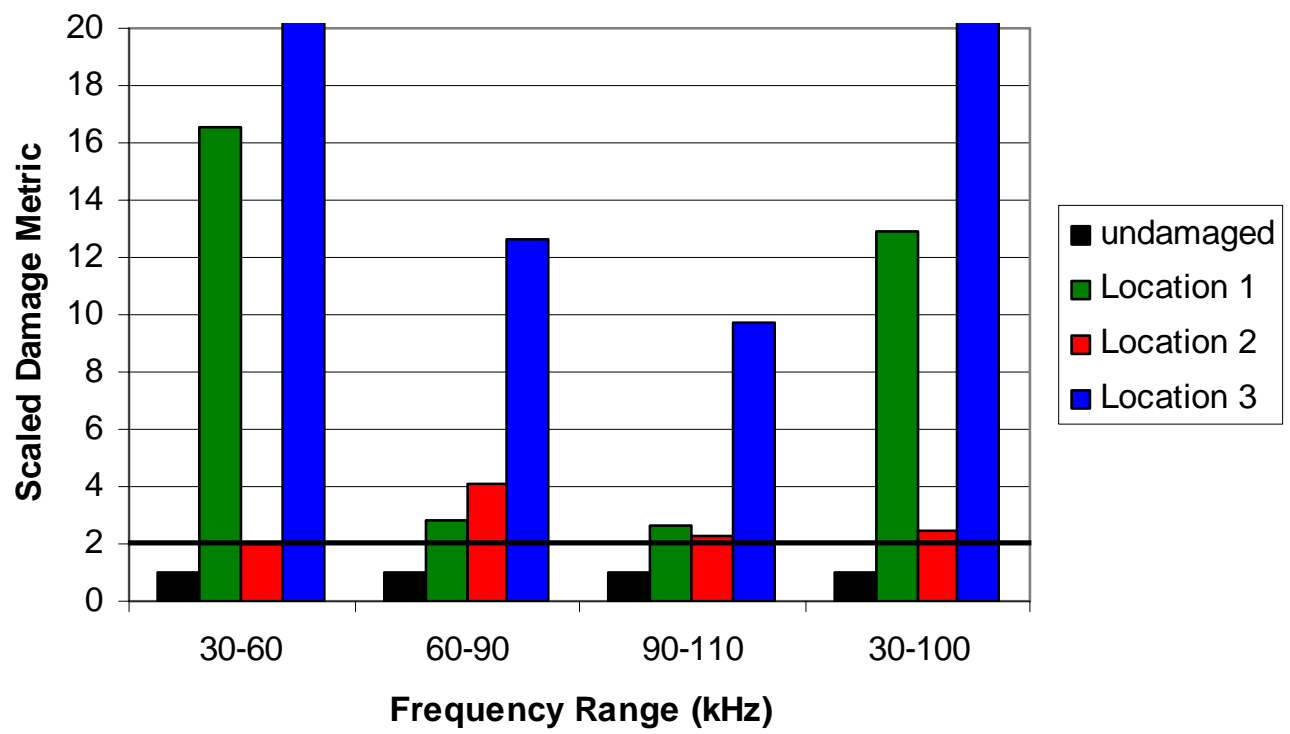

Figure 2.24: Spar, clamp: Damage metrics calculated for several frequency ranges.

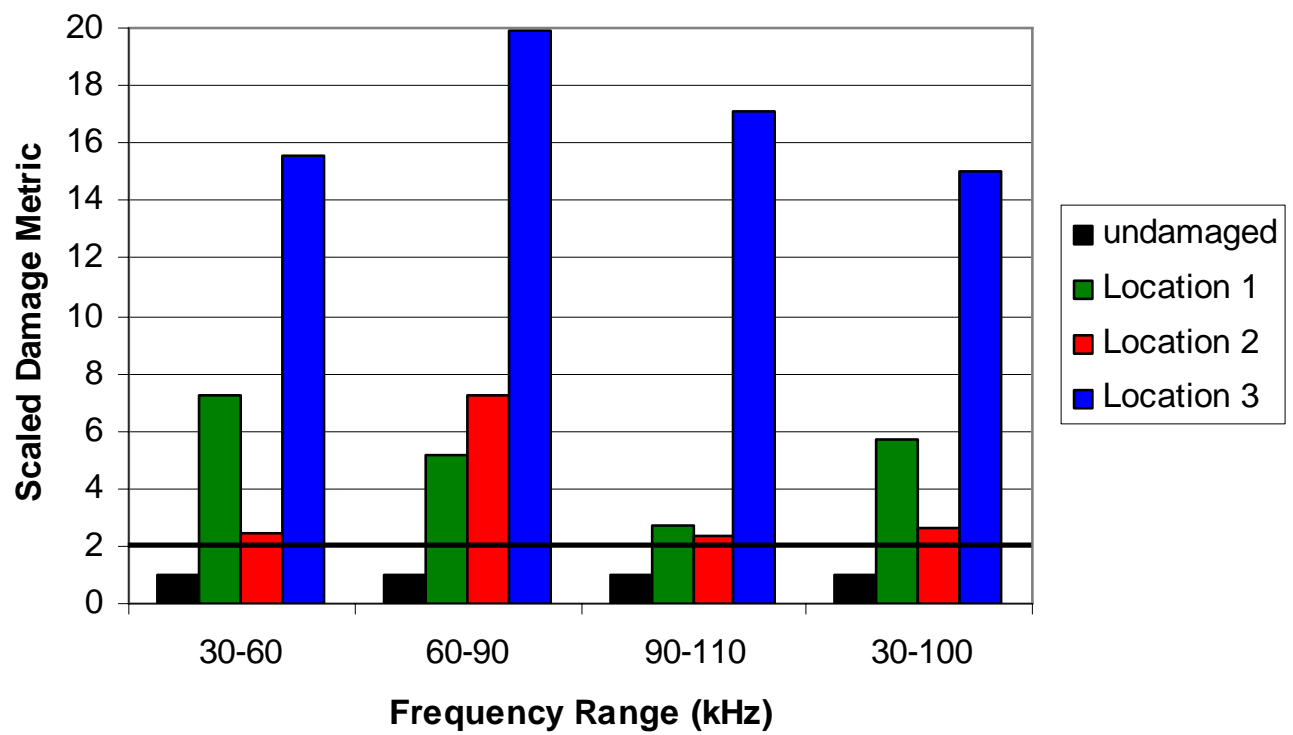

Figure 2.25: Carbon, clamp: Damage metrics calculated for several frequency ranges.

From these results, even though the impedance plots for these high frequency ranges are relatively flat and have low peak density, the damage metrics still indicate damage. None of sensors or frequency ranges is effective in sensing damage in location 2, but all three sensors identify locations 1 and 3 in the $30-60 \mathrm{kHz}$ range 


\subsubsection{Moving mass results}

This section contains the results of tests on the spar and carbon sensors where the 25 gram magnets were used to simulate damage in locations 1,2 , and 3 down the blade section. These tests were run at the sensor end and at locations 13, 25, and 40 inches down the blade. Damage metric results are shown in Figure 2.26 - Figure 2.28. These figures have 4 results for each location. Each color group is for a specific location (green for location 1, red for location 2, and blue for location 3) and as the damage is moved further down the blade $(13,25$, and $40 \mathrm{~cm})$ the shade of the color gets lighter. The test on the spar sensor was run twice and results from both tests are shown.

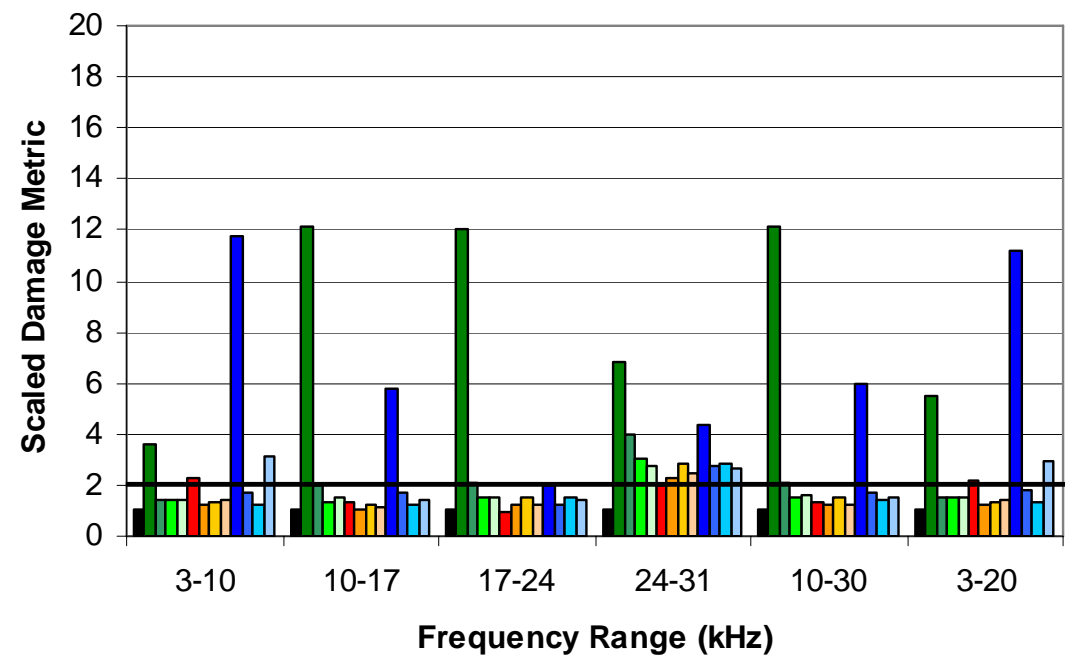

Figure 2.26: Spar, moving mass: Test 1, Damage metrics calculated for several frequency ranges. 


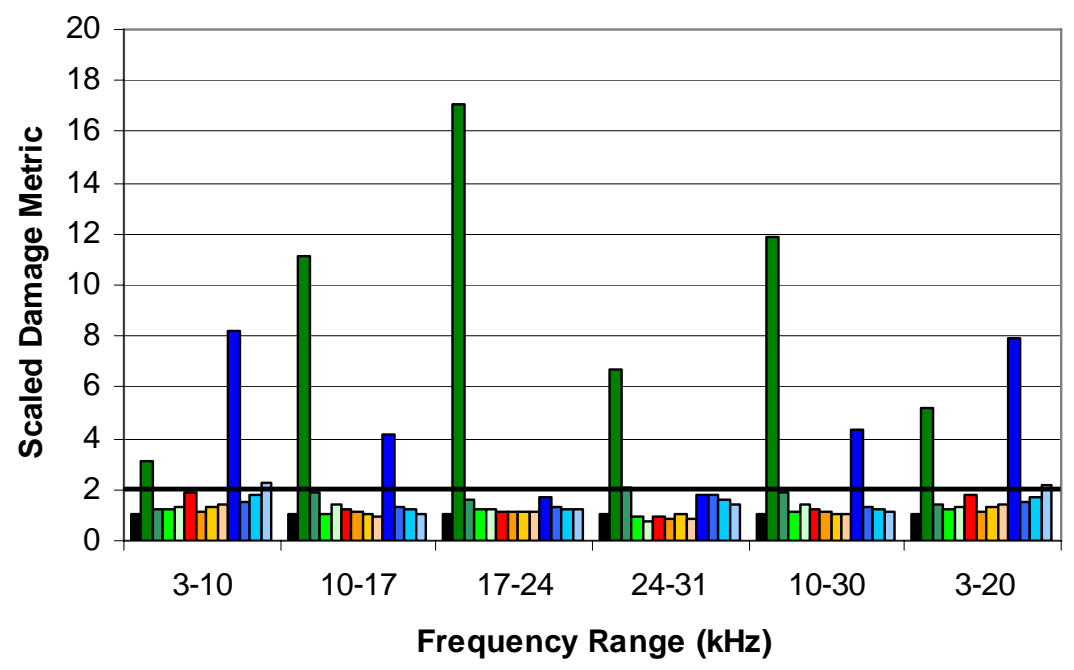

Figure 2.27: Spar, moving mass: Test 2, Damage metrics calculated for several frequency ranges.

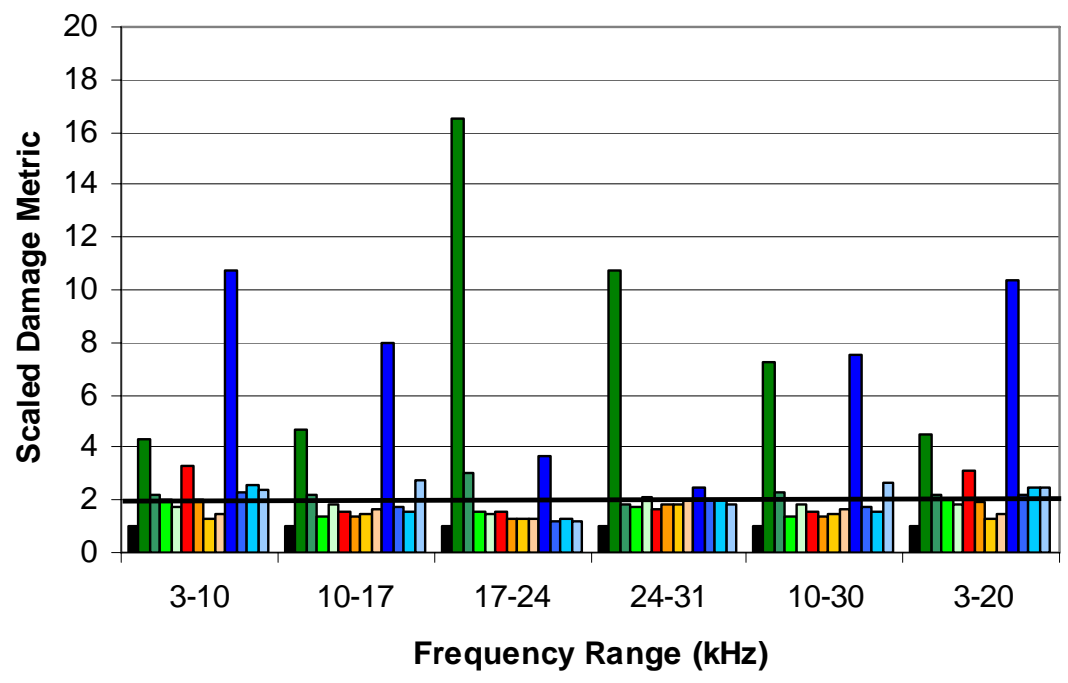

Figure 2.28: Carbon, moving mass: Damage metrics calculated for several frequency ranges.

From these results, neither the spar nor the carbon sensors were able to detect damage at the locations $13 \mathrm{~cm}$ or more down the blade. These locations were nearly all below or right at the damage threshold and as well, no downward trend in the damage metric was observed as the damage was moved further away. Rather the metrics for the locations down the blade seemed to be randomly distributed-an indication that no damage was being sensed. Only damage on the sensor end of the blade was detected, as was already shown in the previous tests. 


\subsubsection{Indirect damage conclusions}

These tests have investigated using IBSHM on the WTB section using indirect damage such as adding mass and changing stiffness (using a C-clamp) at various locations of interest on the blade section. From the results, it does appear that despite the fact that the impedance plots for the blade are relatively flat, the method is able to detect damage in locations 1 and 3 and to a small degree in location 2. Location 2 of course is on the opposite side of the blade and this could be cured by positioning sensors on both sides of the blade. Of concern however, is the low sensing range on the blade. Based on the results, a rough sensing radius for the sensors would be less than $13 \mathrm{~cm}$ for the indirect damage. The methods used to simulate damage however are relatively minutethe mass being added to simulate damage was 25 grams and 215 grams while the blade section weighs roughly $4 \mathrm{~kg}$ and the C-clamp exerted a rather small force. Therefore, it was concluded that the results of the indirect damage testing were promising enough to continue on with actual damage testing of the blade. Also, based on these tests, the frequency ranges chosen to be tested for the actual damage experiments is $10-30 \mathrm{kHz}$ and $30-60 \mathrm{kHz}$. These ranges have the best peak density and have the most consistent results.

\subsection{Actual Damage Results}

Examples of impedance plots from actual damage testing are shown in Figure 2.29 and Figure 2.30. Figure 2.29 displays results from the skin sensor at $10-30 \mathrm{kHz}$ for the progressive separation of the carbon and balsa sections at location 1 on the sensor end of the blade. Figure 2.30 shows the carbon sensor from $10-30 \mathrm{kHz}$ for the increasing diameter holes in the adhesive at location 3 on the sensor end of the blade. Change in the

impedance from baseline to damage states can clearly be seen in both cases. The remaining impedance plots for actual damage testing can be found in Appendix B. 


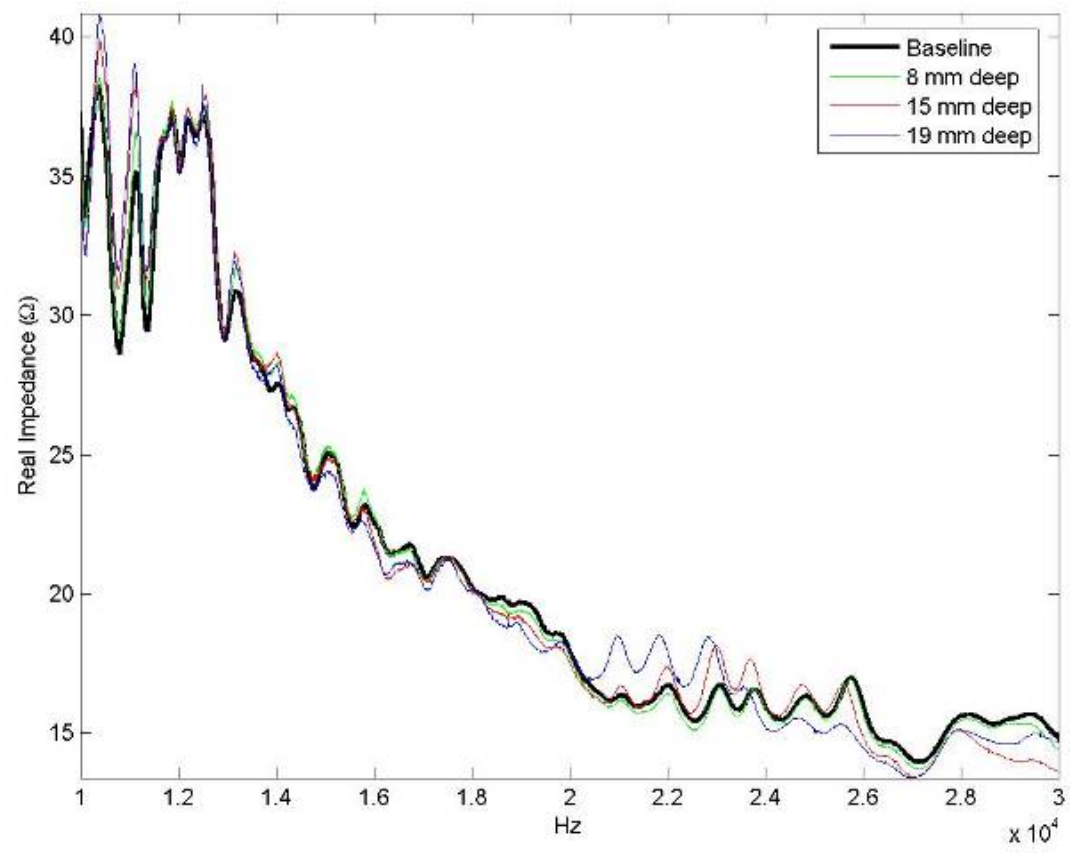

Figure 2.29: Impedance plot for skin sensor, $10-30 \mathrm{kHz}$, location 1 sensor end progressive damage

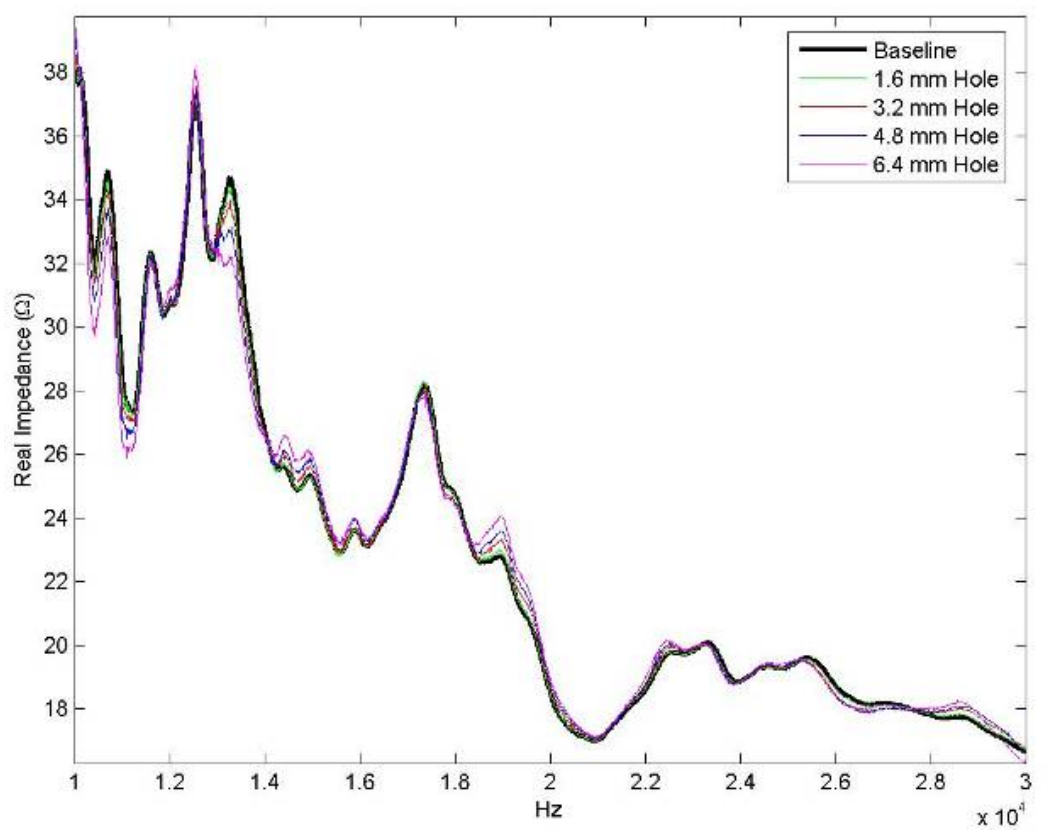

Figure 2.30: Impedance plot for carbon sensor, $10-30 \mathrm{kHz}$, location 3 sensor end progressive damage

\subsubsection{Location 1 results}

Figure 2.31 shows the scaled RMSD damage metrics of the balsa/carbon fiber separation at location 1 for the $10-30 \mathrm{kHz}$ frequency range and Figure 2.32 shows the 30 - 
$60 \mathrm{kHz}$ range. Figure 2.33 shows the $30-60 \mathrm{kHz}$ range for the skin PZT in more detail. Results are shown for both progressive damage at the sensor end of the blade and damage down the length of the blade for all three sensors. For each sensor, the first group is baselines, second is progressively larger gaps between carbon and balsa (darker shade indicates worse damage), and the third is gaps in carbon and balsa $(31,22,12$, and $3 \mathrm{~cm}$ from the sensors, darker shade indicates damage closer to sensor).

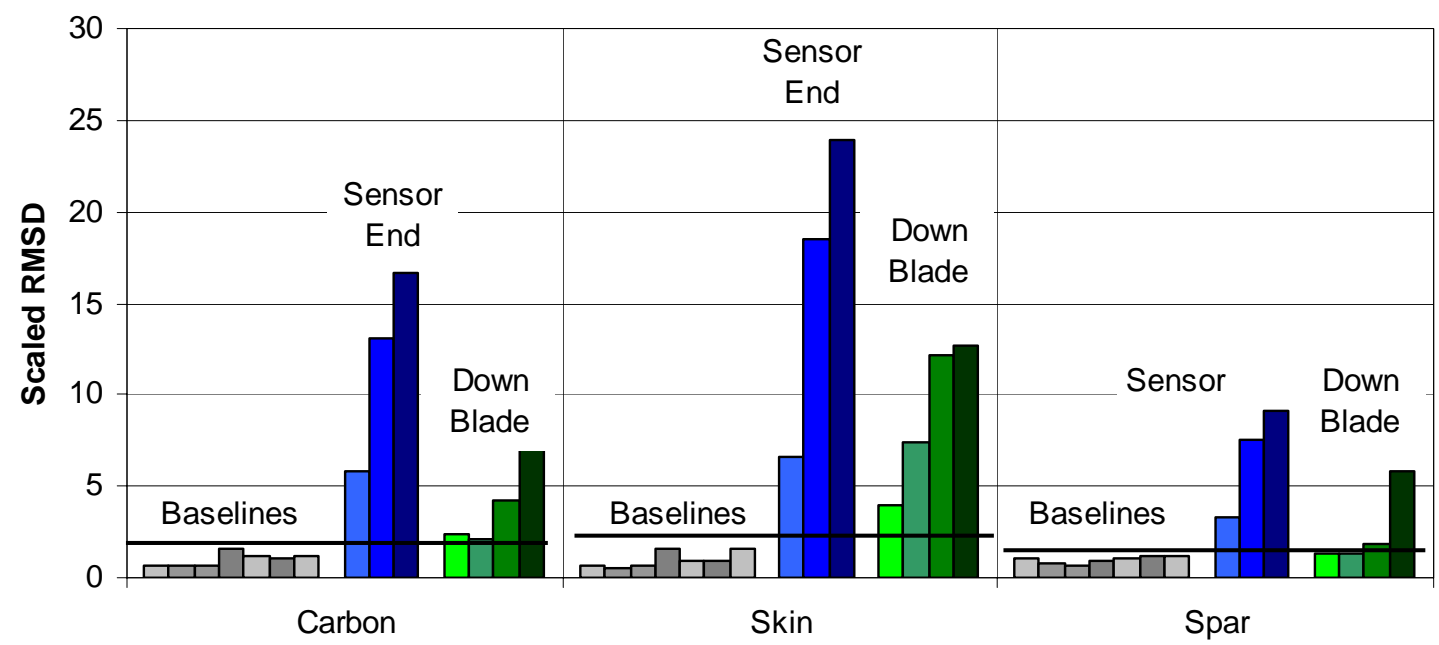

Figure 2.31: Damage metrics for location 1 actual damage tests, $10-30 \mathrm{kHz}$.

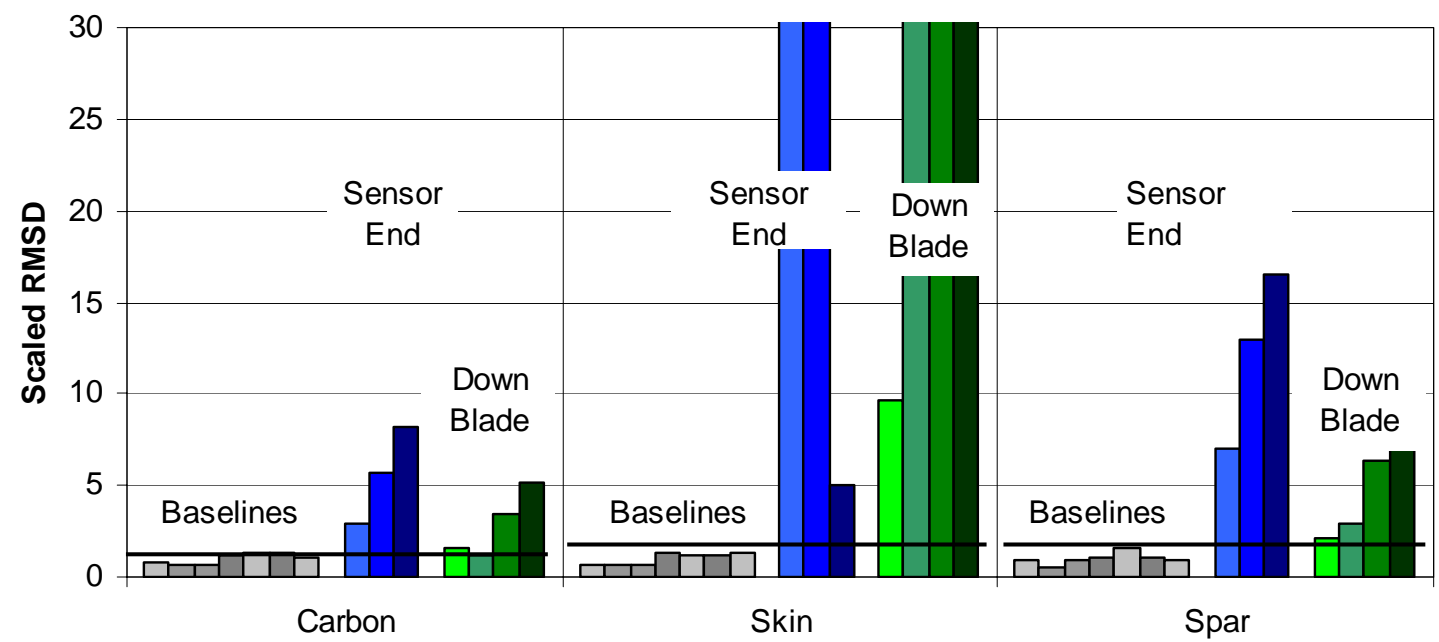

Figure 2.32: Damage metrics for location 1 actual damage tests, 30-60 kHz. 


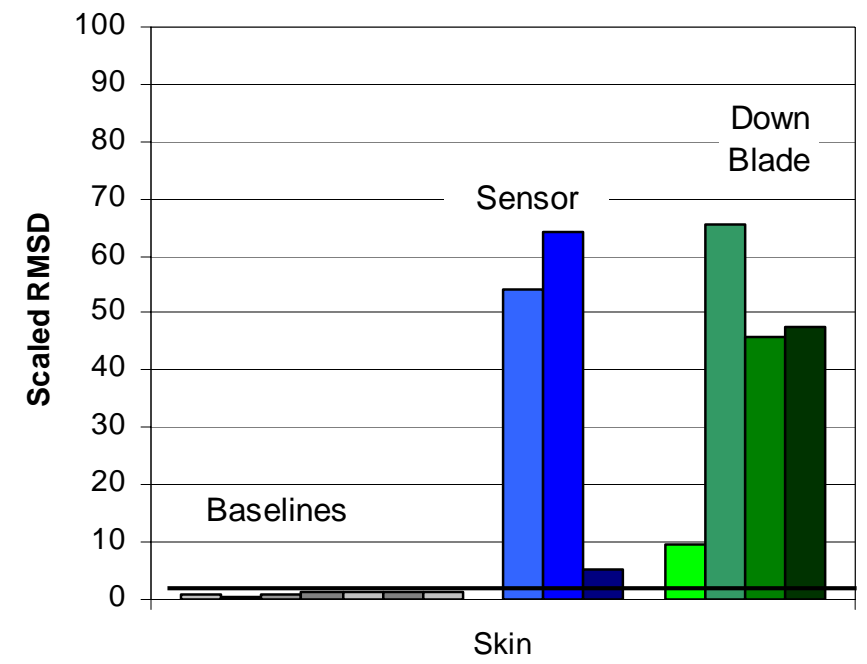

Figure 2.33: Damage metrics for location 1 actual damage tests, 30-60 kHz. Enhanced view of skin PZT

From the $10-30 \mathrm{kHz}$ results, all three sensors were able to effectively sense damage at the sensor end of the blade. As the actual damage in the blade increased, the damage metric also increased. For the testing down the length of the blade, the skin sensor was able to effectively sense the damage all the way to $31 \mathrm{~cm}$ down the blade, and a clear upward trend is seen in the metrics as the damage nears the sensor. The carbon sensor had significant damage metrics for up to $12 \mathrm{~cm}$ down the blade, while the spar sensor only effectively sensed the damage $3.2 \mathrm{~cm}$ down the blade. The relative success of each sensor makes sense due to their proximity to the damage location. The skin sensor had the best results, which would be expected due to its close proximity to location 1. The spar sensor had the least success, but it has adhesive within its path to the damage.

The $30-60 \mathrm{kHz}$ range results are similar to those of the $10-30 \mathrm{kHz}$ range except that the spar sensor seems to have more sensitivity and a better sensing range. Looking at the impedance plot in Appendix B however, these results seem suspect due to the low peak density in this range and the relatively small difference between impedance plots. Also, as seen in Figure 2.33, the skin patch has a very high sensitivity in this range, however the results seem inconsistent. The metrics do not follow increasing trends with 
increasing damage or as damage gets closer to the sensor. Again, these inconsistencies seem to be the product of lower peak density than in the $10-30 \mathrm{kHz}$ range.

\subsubsection{Location 3 results}

Figure 2.34 shows the scaled RMSD damage metrics of the holes in the adhesive at location 3 for the $10-30 \mathrm{kHz}$ frequency range and Figure 2.35 shows the $30-60 \mathrm{kHz}$ range. Figure 2.36 shows the results of the spar PZT from $30-60 \mathrm{kHz}$ in more detail. Results are shown for both progressive damage at the sensor end of the blade and damage down the length of the blade for all three sensors. For each sensor, the first group is baselines, second is progressively larger holes in adhesive as the shade gets darker, and the third is holes in adhesive getting closer to the sensors as shade gets darker $(28,20,13$, 5 , and $0 \mathrm{~cm}$ from the sensors).

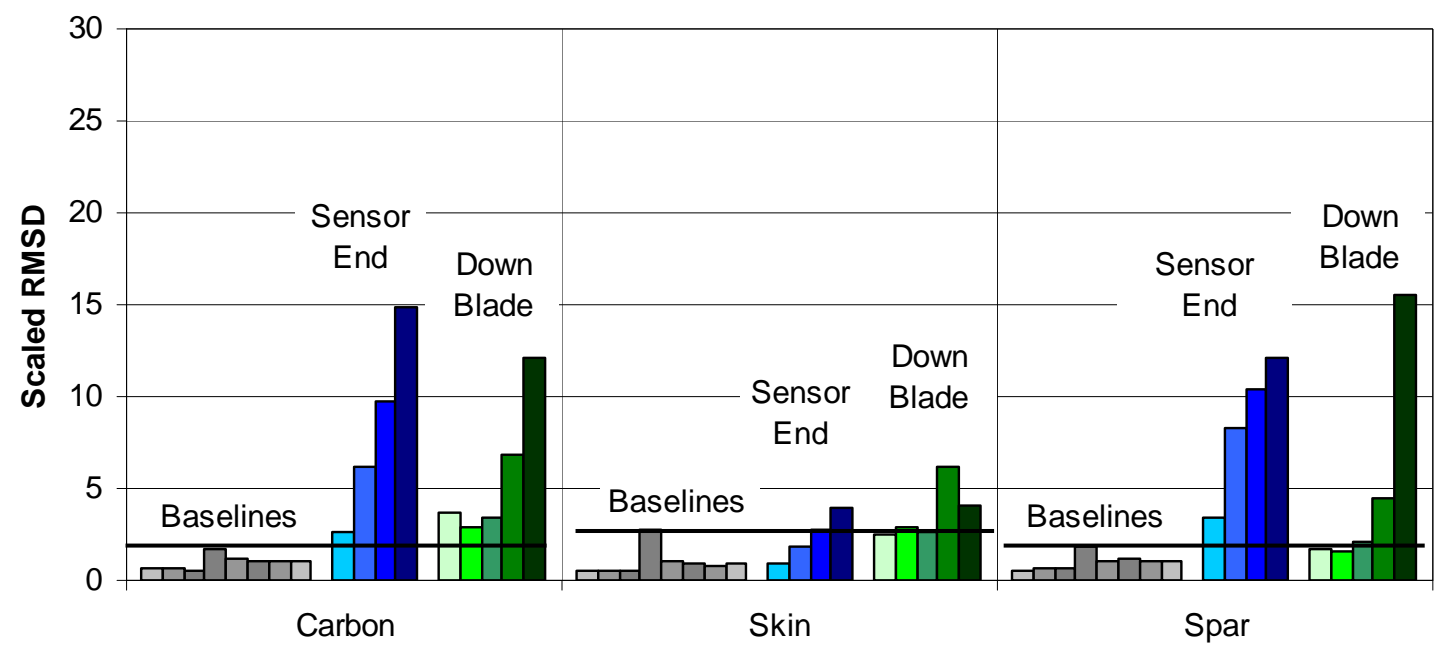

Figure 2.34: Damage metrics for location 3 actual damage tests, $10-30 \mathrm{kHz}$. 


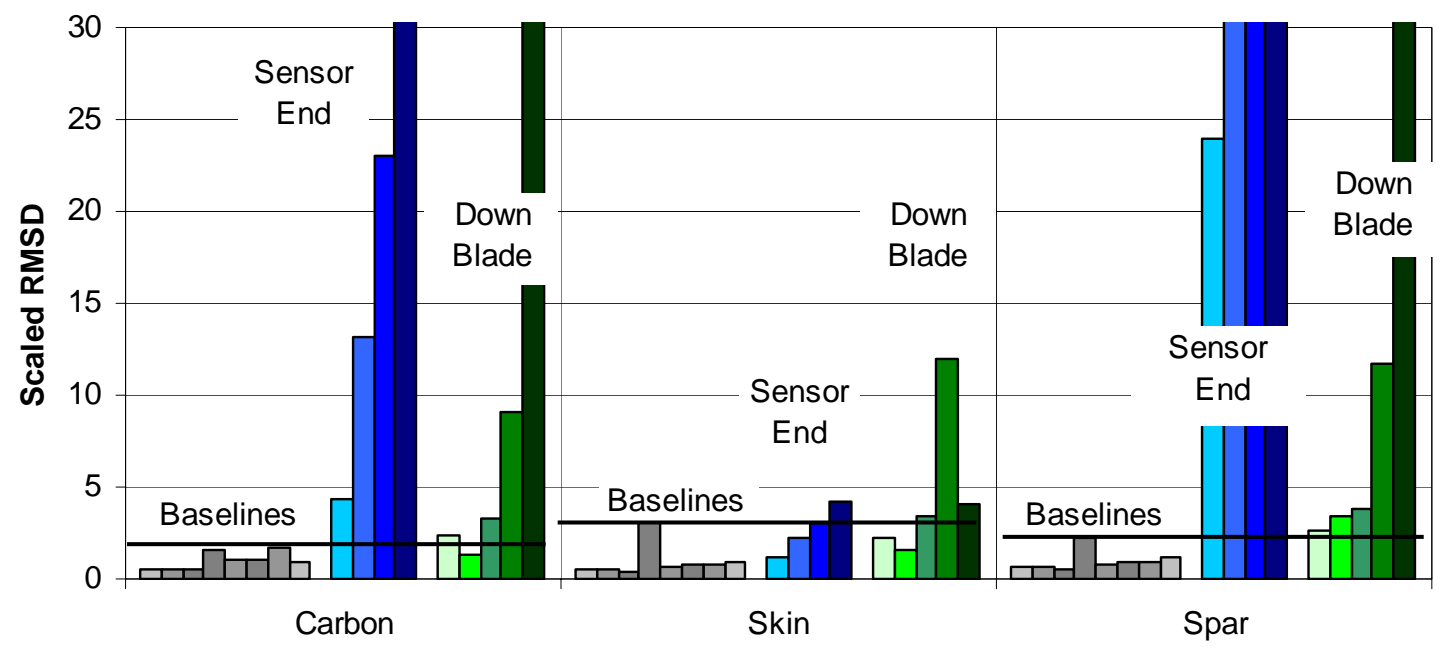

Figure 2.35: Damage metrics for location 3 actual damage tests, 30-60 kHz.

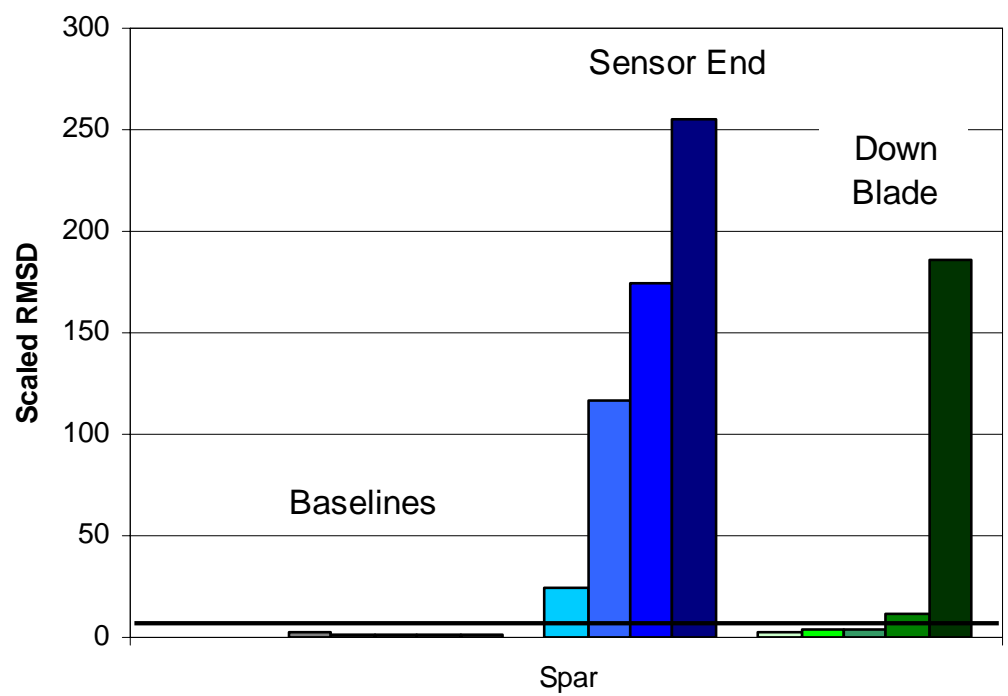

Figure 2.36: Damage metrics for location 3 actual damage tests, $30-60 \mathrm{kHz}$. Enhanced view for the spar PZT

From the $10-30 \mathrm{kHz}$ results, all three sensors were able to effectively sense damage at the sensor end of the blade with an increasing trend in the damage metric as the damage worsened, with the exception of the skin sensor not effectively sensing the smallest size hole. For the testing down the length of the blade, the carbon and spar sensor had the most success, with a downward trend in damage metric for the 0,5 , and 13 
$\mathrm{cm}$ tests. The carbon sensor seemed to be able to sense the 20 and $28 \mathrm{~cm}$ damage, but the downward trend was not preserved.

The $30-60 \mathrm{kHz}$ range for location 3 results are similar to the $10-30 \mathrm{kHz}$ range. Overall, the damage metrics are relatively higher than the $10-30 \mathrm{kHz}$ range, very much so for the spar PZT as seen in Figure 2.36. Even though these results are higher, they appear to be just scaled up from the $10-30 \mathrm{kHz}$ results and do not represent much of an increased sensitivity.

\subsubsection{Actual damage conclusions}

Based on the results of the actual damage testing, all of the sensors were able to sense actual damage on the sensor end of the blade. The damage metrics for these tests also had an increasing trend as damage increased. For the damage down the length of the blade, the sensing range was around $13 \mathrm{~cm}$ for the adhesive holes and $30 \mathrm{~cm}$ for the balsa/carbon fiber gap, using the best sensors. The optimal sensor for these cases was the skin sensor for location 1 and the carbon or spar sensor for location 3. Overall, the 10-30 $\mathrm{kHz}$ and $30-60 \mathrm{kHz}$ ranges produced similar results. However, despite having a seemingly better sensitivity, the $30-60 \mathrm{kHz}$ range produced less consistent results.

\subsection{Conclusions}

Testing on a CX-100 WTB section has been performed to help sort out the feasibility of using IBSHM on WTBs. The goal was to see if the method is able to detect damage on the blade and to determine the limits of sensing. The method of introducing damage in a controlled way was to add mass and change stiffness as well as inducting actual damage at various locations of interest on the blade section. Damage mechanisms were induced both on the sensor end as well as down the length of the blade.

From the results, the impedance method was able to detect damage on the blade section. The sensing range is around $10-30 \mathrm{~cm}$ depending on the sensor and type of 
damage. Considering the size of WTBs, this may seem to be a rather small range. Still, it seems that IBSHM is a promising method to use on blades either in critical locations or in conjunction with other SHM methods which both utilized the same PZT patches.

The results of the indirect and direct damage testing show that the spar PZT location and $10-30 \mathrm{kHz}$ impedance range are the best parameters for further tests. This location and range offers the best overall sensitivity to all of the damage locations as well as the most consistent results. Based on the results of the indirect and direct damage testing on the CX-100, the impedance method was also tested on a TX-100, a blade somewhat similar to the CX-100, during a fatigue test at NREL's NWTC. This work is presented in Chapter 4. 


\section{CHAPTER 3 High-Frequency Response Function Structural Health Monitoring of CX-100 Wind Turbine Blade Section}

\subsection{Introduction}

In addition to testing IBSHM on the CX-100 WTB section from SNL presented in Chapter 2, the High-Frequency Response Function (HFRF) method of SHM was also investigated on the blade section. This form of SHM was recommended by Gyuhae Park of Los Alamos National Laboratory as an alternative method to compare to IBSHM on the blade section. The HFRF method was validated as a form of damage detection on composite plates by Park et al. (2005). It is also similar to but uses higher frequency than the SHM method used by Zayas et al. (2007) during fatigue testing of the CX-100 and similar 3X-100 blades, as discussed in Chapter 1. Therefore, the HFRF method seemed to be an ideal method to compare to the IBSHM results on the CX-100 WTB section given its validation on composite structures and its use on testing of the CX-100. Additionally, with PZTs already mounted to the structure from the IBSHM testing, incorporating this type of testing on the WTB section was straightforward. Like the IBSHM tests, the HFRF testing included both indirect and direct forms of damage. The HFRF indirect experiments were performed after the IBSHM, but for the actual damage testing, HFRF testing was run in unison with IBSHM.

The HFRF method makes use of the transfer function between two MFCs or PZTs bonded to a structure but in a higher frequency range than typically is used for modal testing. Park et al. (2005) bonded both PZTs and MFCs to a composite plate and then induced delamination in the plate by firing a projectile at it from a gas gun. Baseline and damaged FRFs were compared by calculating a damage metric of one minus the correlation coefficient, which is related to RMSD, as used in Chapter 2 for IBSHM. The frequency range used was $5-20 \mathrm{kHz}$ and the damage introduced into the plate was 
detected by the method and validated using ultrasonic scanning methods. The frequency range less than $5 \mathrm{kHz}$ was left out of the analysis because this often contains information affected by boundary conditions. The upper limit of $20 \mathrm{kHz}$ corresponds to the upper limit of the equipment available to perform this experiment; therefore the frequency range of 5-20 kHz which was used in Park's work was also selected to be used to test the WTB section. Additionally, it is close to the range found to work the best for IBSHM, $10-30 \mathrm{kHz}$.

\subsection{Experimental setup and procedure}

For the HFRF method, transfer functions are needed between two PZTs bonded to the CX-100 section. The same PZTs used for IBSHM tests, in Figure 2.5, were used for HFRF tests. The three PZTs were used in three input/output pairs for the transfer functions: skin/spar, skin/carbon, and spar/carbon. The PZTs were excited and transfer functions between the pairs were measured using a model 20-42 dsp-Siglab digital signal processor controlled with a laptop running Siglab version 3.26 software. The input/output sampling used was a $20 \mathrm{kHz}$ bandwidth with a Hanning window applied to 8192 samples. Only the $5-20 \mathrm{kHz}$ portion of the data was used to determine damage.

The HFRF testing was conducted in a similar manner to the IBSHM testing from Chapter 2. The indirect damage testing was performed at a different time than the IBSHM tests, but the same procedure was used as described in section 2.2.2. Locations 1,2 , and 3 were tested at the sensing end of the blade with 25 gram magnets for added mass and C-clamp for added stiffness and mass. Also, added mass was tested down the length of the blade at $0,13,25,40 \mathrm{~cm}$. First, 5 baselines transfer functions were obtained and then 1 transfer function was obtained for each damage case. This set of experiments was tested using two input excitations. The first was a 1 volt max random signal excitation with $20 \mathrm{kHz}$ bandwidth sampled at $51.2 \mathrm{kHz}$. The second was a 5 volt max, 5$20 \mathrm{kHz}$ chirp with 0.1 second on time, 0.01 second off time with 5120 samples. 
Actual damage testing with HFRF was conducted in unison with the IBSHM testing described in section 2.2.3. For the input excitation, a 3.5 volt max random signal excitation with $20 \mathrm{kHz}$ bandwidth sampled at $51.2 \mathrm{kHz}$ was used.

The test procedure used for the HFRF damage detection is the same as the IBSHM tests described in section 2.2.1. The transfer functions produced by the HFRF testing are similar to the impedance signatures used in the impedance method. Multiple baseline transfer functions are compared to the damaged transfer functions frequency by frequency using a RMSD damage metric. Results are scaled so that the average baseline metric equals one and a threshold value is calculated statistically from the variation of the baseline measurements.

\subsection{Indirect damage results}

The baseline transfer function of the skin/carbon PZT combination is shown in Figure 3.1 along with its coherence for the 1 volt random signal excitation. The remaining baseline transfer functions along with their coherence are shown in Appendix C. The shape of the transfer function is similar to that of the impedance plots of the impedance method. Coherence is acceptable except for at resonances and antiresonances. As seen in Appendix $\mathrm{C}$, the coherence improves a small amount for the 5 volt input compared to the 1 volt input. All of the transfer functions have several peaks in the $5-20 \mathrm{kHz}$ range. 

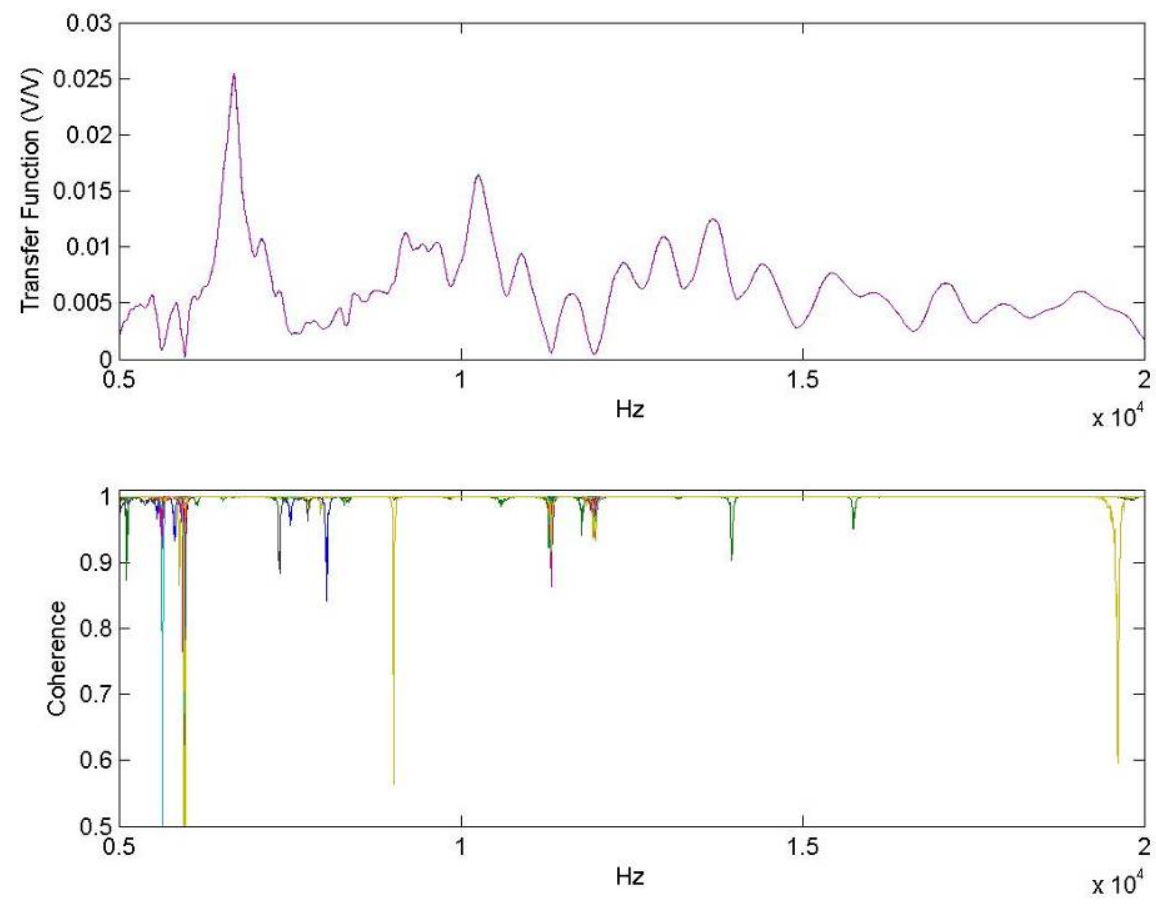

Figure 3.1: Baseline transfer function and coherence, skin/carbon PZTs, $1 \mathrm{~V}$ random signal input

\subsubsection{Added mass and stiffness results}

This section contains the results of the HFRF method tests using indirect damage on the sensing end of the blade. Examples of the transfer function plots are shown in the following figures. Figure 3.2 shows the transfer function of the spar/carbon PZT combination for the added stiffness test with the 1 volt random excitation signal. Figure 3.3 shows the transfer function of the skin/carbon PZT combination for the added mass test with the 5 volt random excitation signal. Change from baseline to damage states can clearly be seen in the transfer functions. The remaining transfer function plots for the HFRF indirect damage tests can be found in Appendix C. 


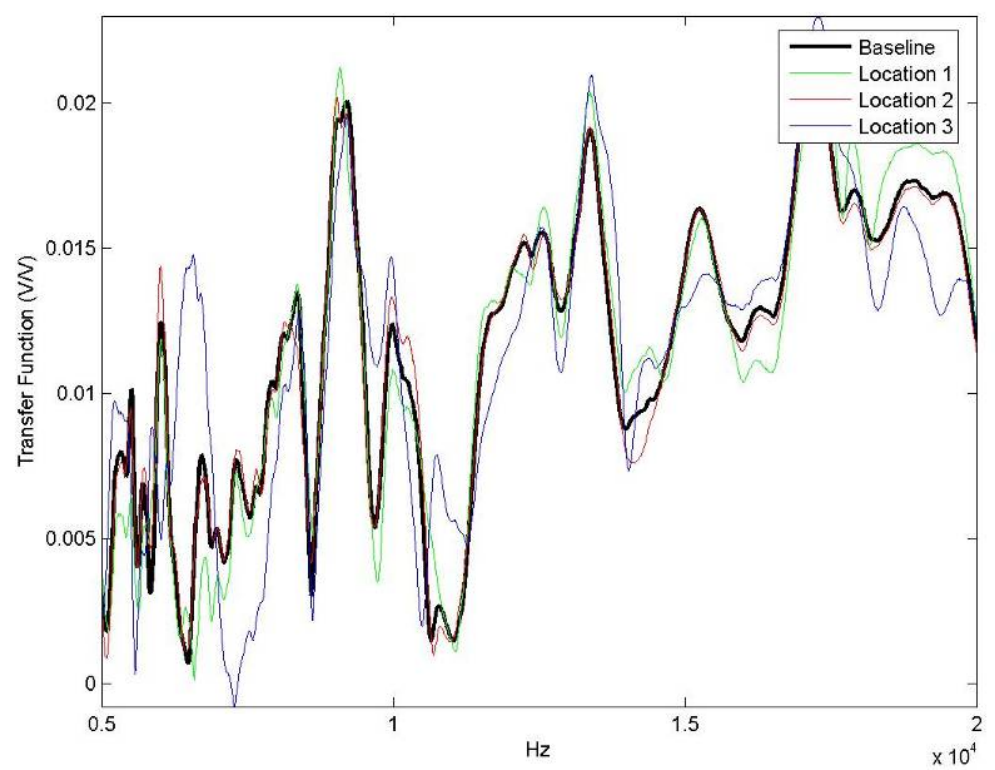

Figure 3.2: $\mathrm{Spar} /$ Carbon transfer function, $1 \mathrm{~V}$ random signal, added stiffness

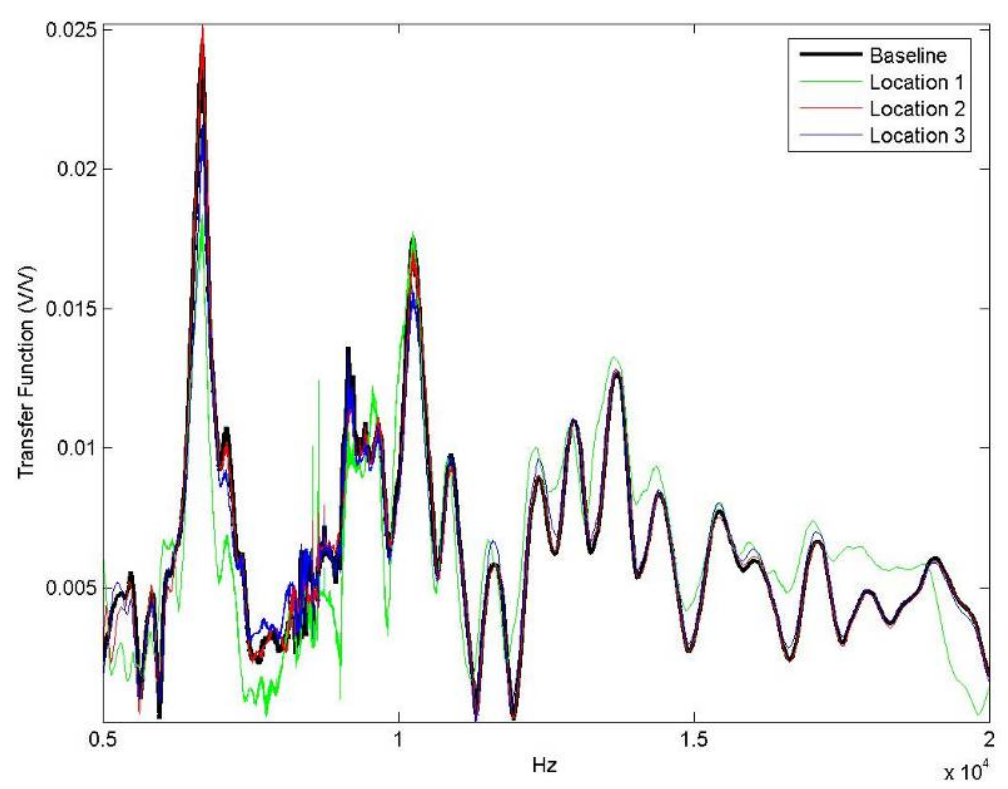

Figure 3.3: Skin/Carbon transfer function, $5 \mathrm{~V}$ chirp signal, added mass

The results of the added mass and stiffness HFRF indirect damage tests at the sensor end of the blade for locations 1, 2, and 3 for the three PZT input/output combinations follow. Figure 3.4 shows the results using the 1 volt random excitation signal while Figure 3.5 contains the results using the 5 volt chirp signal. Like the results 
for IBSHM in Chapter 2, in the damage metric charts each group of damage metrics are scaled so that the average of baseline metrics is equal to one.

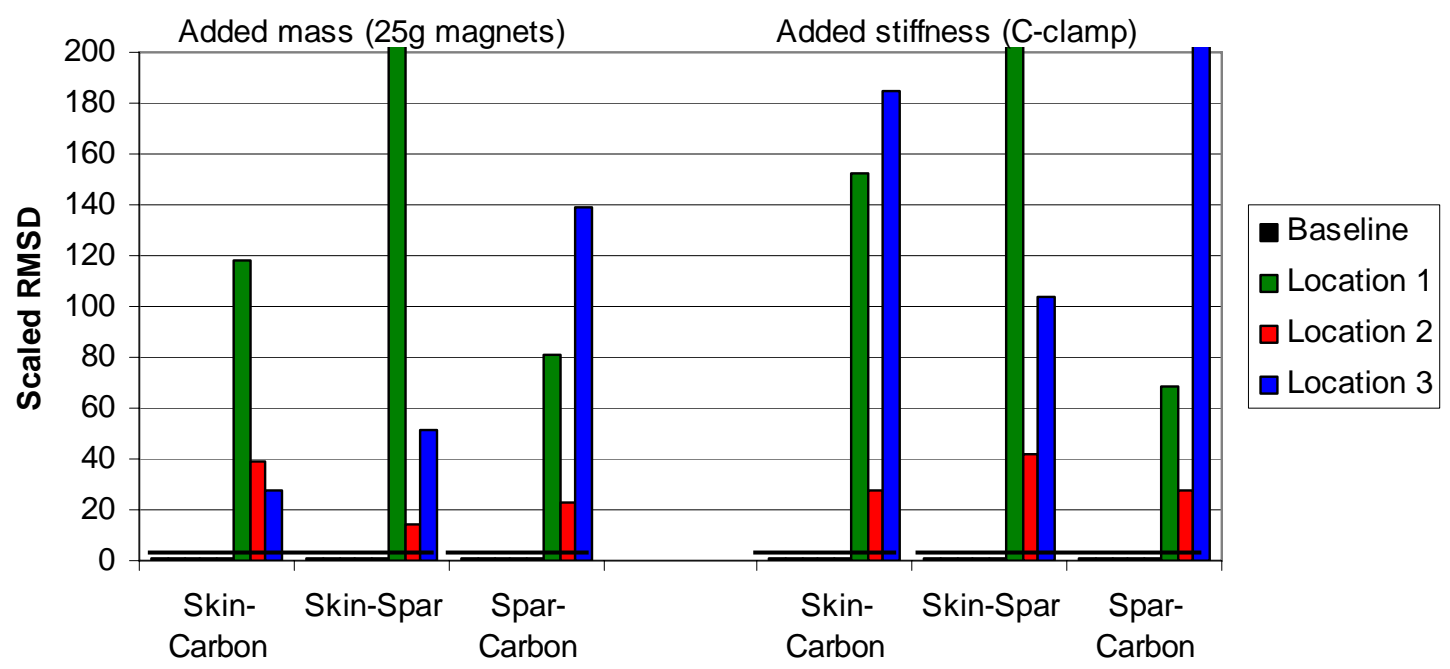

Figure 3.4: Added mass (25g) and stiffness (C-clamp) at sensor end. 1V random signal input

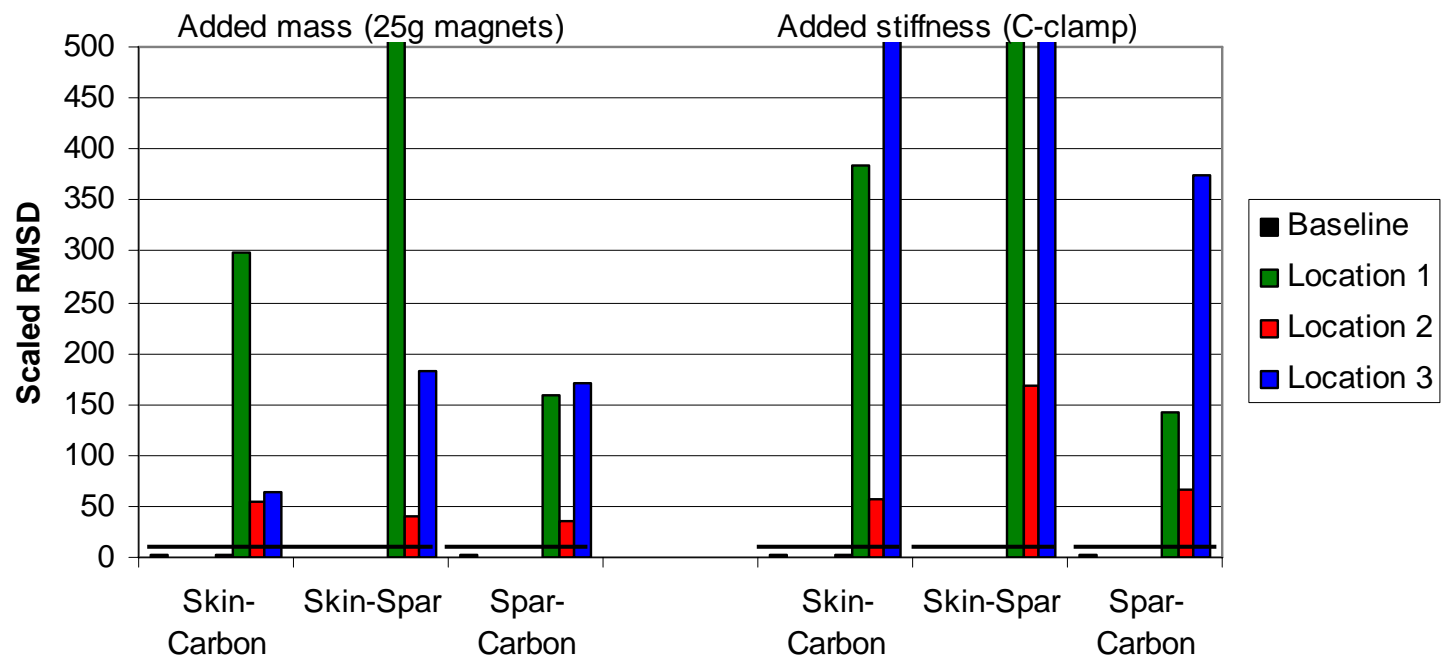

Figure 3.5: Added mass (25g) and stiffness (C-clamp) at sensor end. 5V chirp signal input

From the indirect damage at the sensor end tests, it is clear that the results using 1 volt random signal are similar to that of the 5 volt chirp. The two sets of results generally follow the same trend with the difference being that the 5 volt damage metrics are higher than the 1 volt set. This increased sensitivity due to the higher excitation voltage is the result that is to be expected. Also, all input/output PZT combinations seem capable of 
sensing damage in all three locations for both the added mass and added stiffness indirect damage, including but to a lesser degree location 2 on the opposite side of the blade from the sensors.

\subsubsection{Moving mass results}

This section contains the results of tests on all three input/output PZT combinations where the 25 gram magnets were used to simulate damage in locations 1,2, and 3 down the blade section. These tests were run at the sensor end and at locations 13, 25, and 40 inches down the blade. Damage metric results are shown in Figure 3.6 and Figure 3.7 for the 1 volt random signal and 5 volt chirp input signals respectively. These figures have 4 results for each location. Each color group is for a specific location (green for location 1, red for location 2, and blue for location 3) and as the damage is moved further down the blade $(13,25$, and $40 \mathrm{~cm})$ the shade of the color gets lighter.

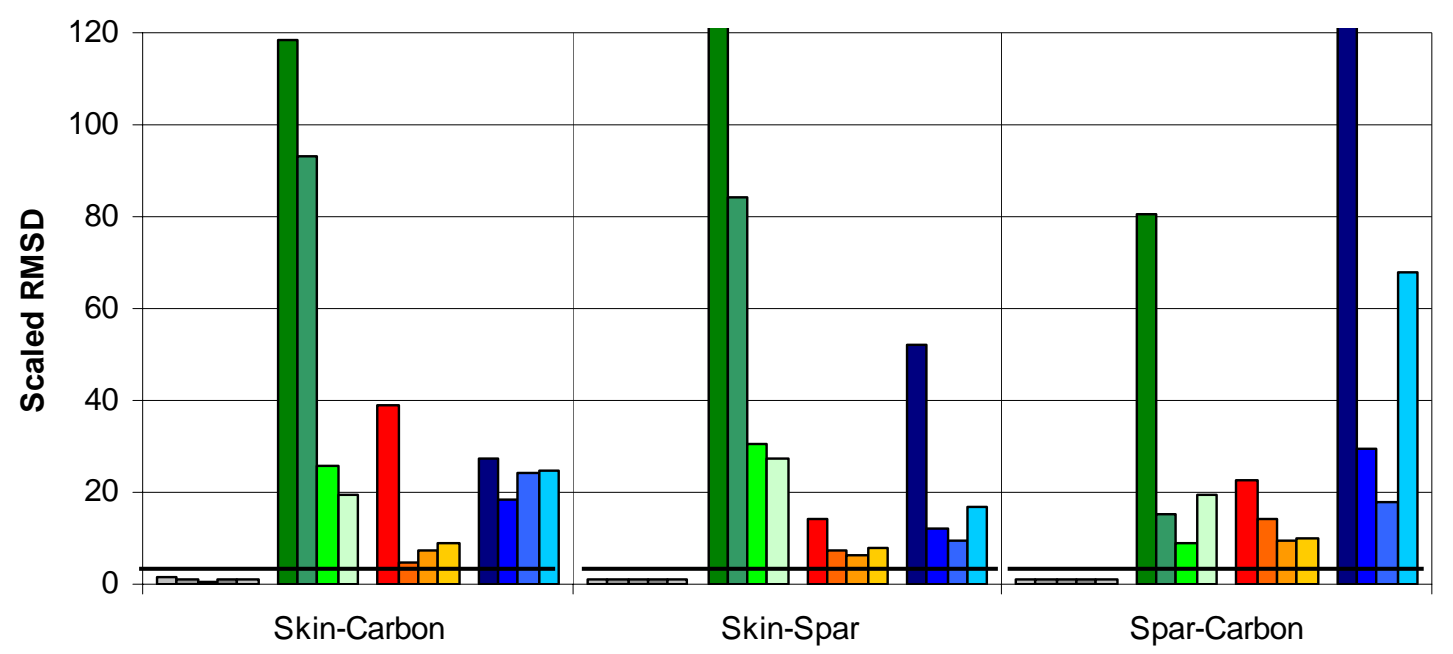

Figure 3.6: Moving mass results. $1 \mathrm{~V}$ random signal input. 


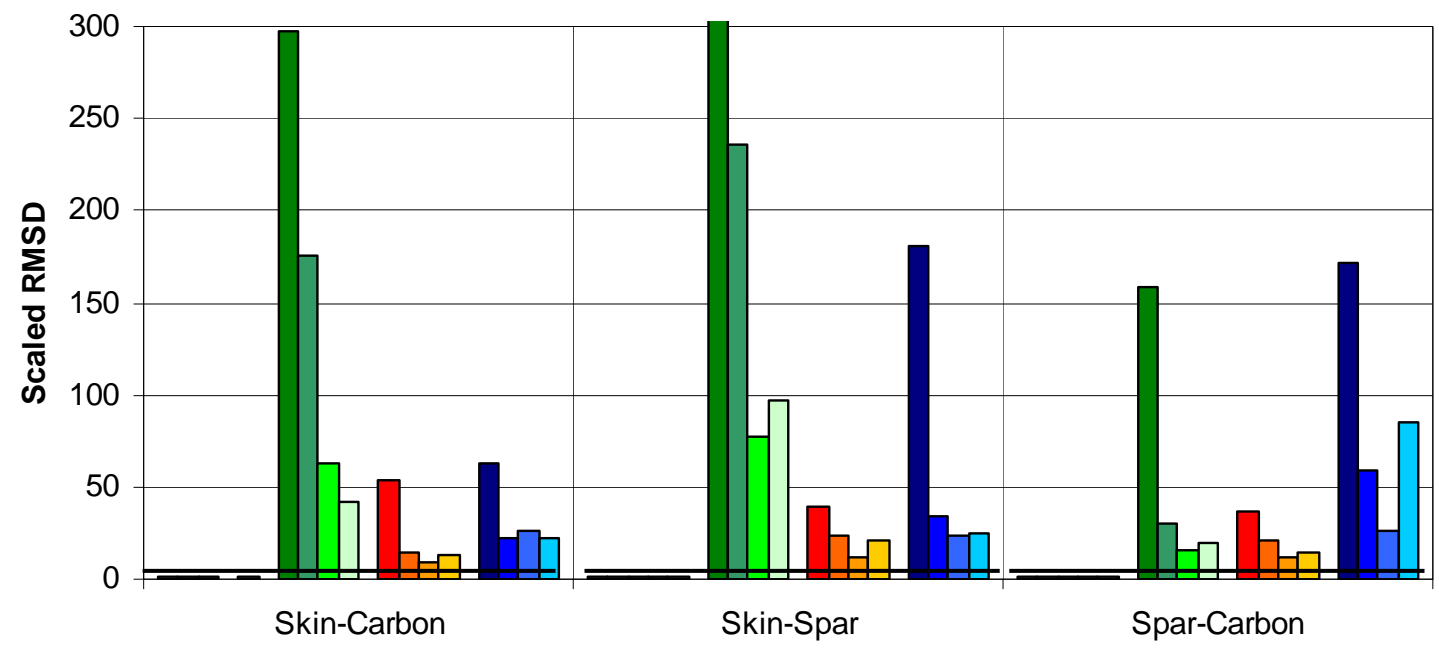

Figure 3.7: Moving mass results. 5V chirp signal input.

Similar to the results of the sensor end indirect damage tests for HFRF, the moving mass test results show results the same for 1 volt and 5 volt excitation signals with the difference being that the 5 volt results are close to being a scaled up version of the 1 volt results. An interesting aspect of these results is that as the damage is moved down the blade in most all of the cases, the damage metric decreases, as expected, but then increases. It looks as though after the 0 or $5 \mathrm{~cm}$ case the damage metric is simply random. This could possibly be due to the slightly lower frequency of this method making it sense a global change in the structure, but not necessarily damage. It seems that the statistically determined value for threshold may not be appropriate in this case and it may be possible that qualitative threshold should be set significantly higher. Given a higher qualitative threshold, the sensing range or the HFRF method for indirect damage is around only $5 \mathrm{~cm}$.

\subsubsection{Indirect damage conclusions}

Indirect forms of damage were used to test the HFRF method on the CX-100 WTB section. In general, the results for both input excitation types were consistent, with the only difference being that the damage metrics were greater for the 5 volt chirp signal. The results of the moving mass tests reveal that the statistical damage metric threshold 
may be too low for evaluating damage. If the threshold was set to a more appropriate value (approximately 20 for the 1 volt signal and 50 for the 5 volt signal) the sensing range of the method would be less than $5 \mathrm{~cm}$. Also, applying this to the previous sensing end results, the method would not be effectively sensing damage at location 2 on the opposite side of the blade.

\subsection{Actual damage results}

The results of the actual damage tests in location 1 and location 3 on the blade section are presented here. Examples of the transfer functions from actual damage testing are shown in the following figures. Figure 3.8 displays results from the spar/carbon PZT combination for split between the carbon fiber and balsa at the sensor end at location 1 . Figure 3.9 shows skin/carbon combination for the increasing diameter holes in the adhesive at location 3 on the sensor end of the blade. Change in the transfer function from baseline to damage states can clearly be seen in both cases. The remaining transfer functions for actual damage testing with the HFRF method can be found in Appendix D.

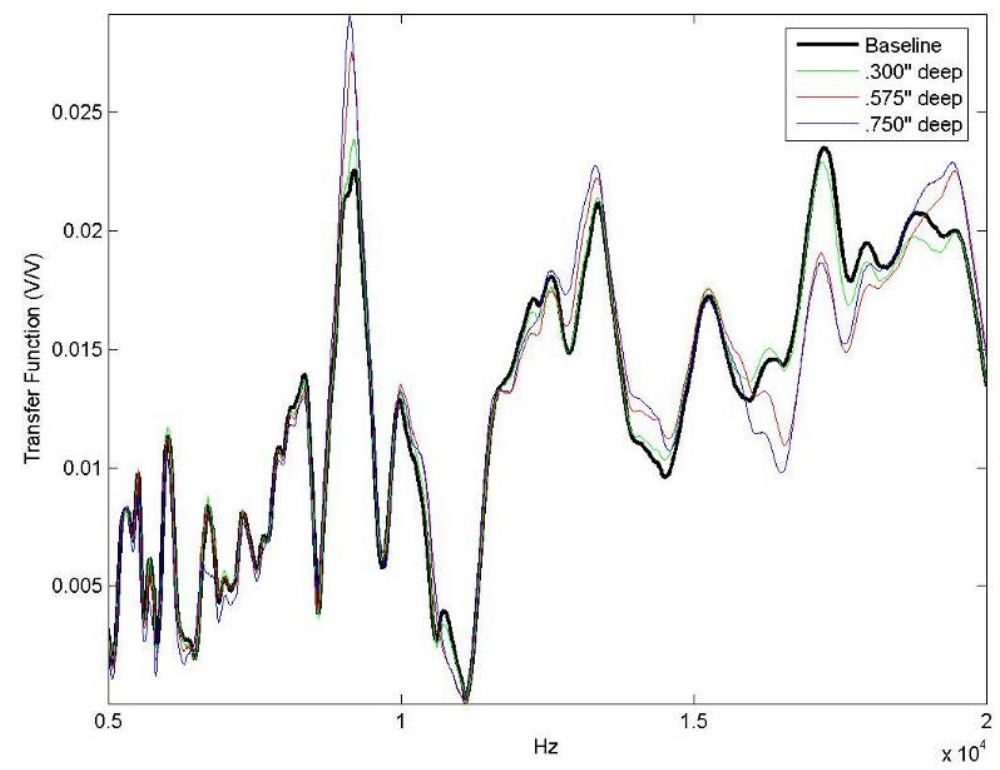

Figure 3.8: Spar/carbon PZT combination, location 1 split at sensor end 


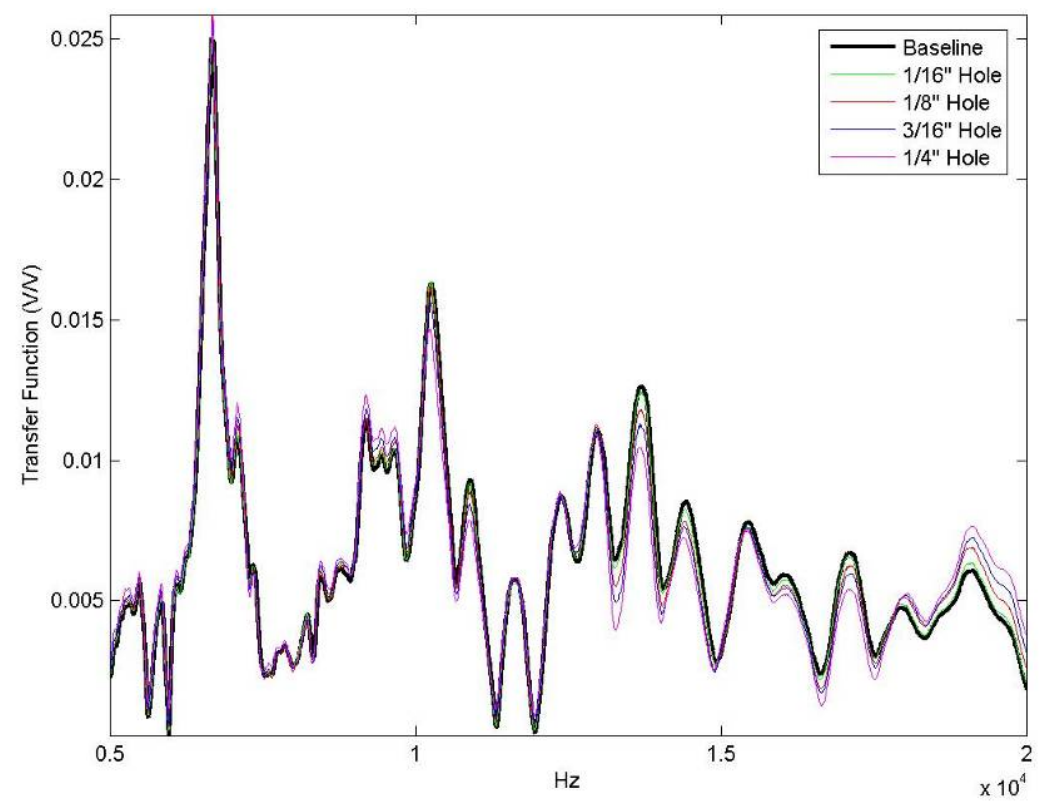

Figure 3.9: Skin/carbon PZT combination, location 3 holes at sensor end

\subsubsection{Location 1 results}

This section contains the results of the HFRF method detecting damage at the balsa/carbon fiber separation at location 1. Figure 3.10 shows the scaled RMSD damage metrics of these tests for both progressive damage at the sensor end of the blade and damage down the length of the blade for all three PZT combinations. For each PZT combination, the first group is baselines, second is progressively larger gaps between carbon and balsa (darker shade indicates worse damage), and the third is gaps in carbon and balsa $(31,22,12$, and $3 \mathrm{~cm}$ from the sensors, darker shade indicates damage closer to sensor). Figure 3.11 shows a more detailed view of the skin/spar damage metrics. 


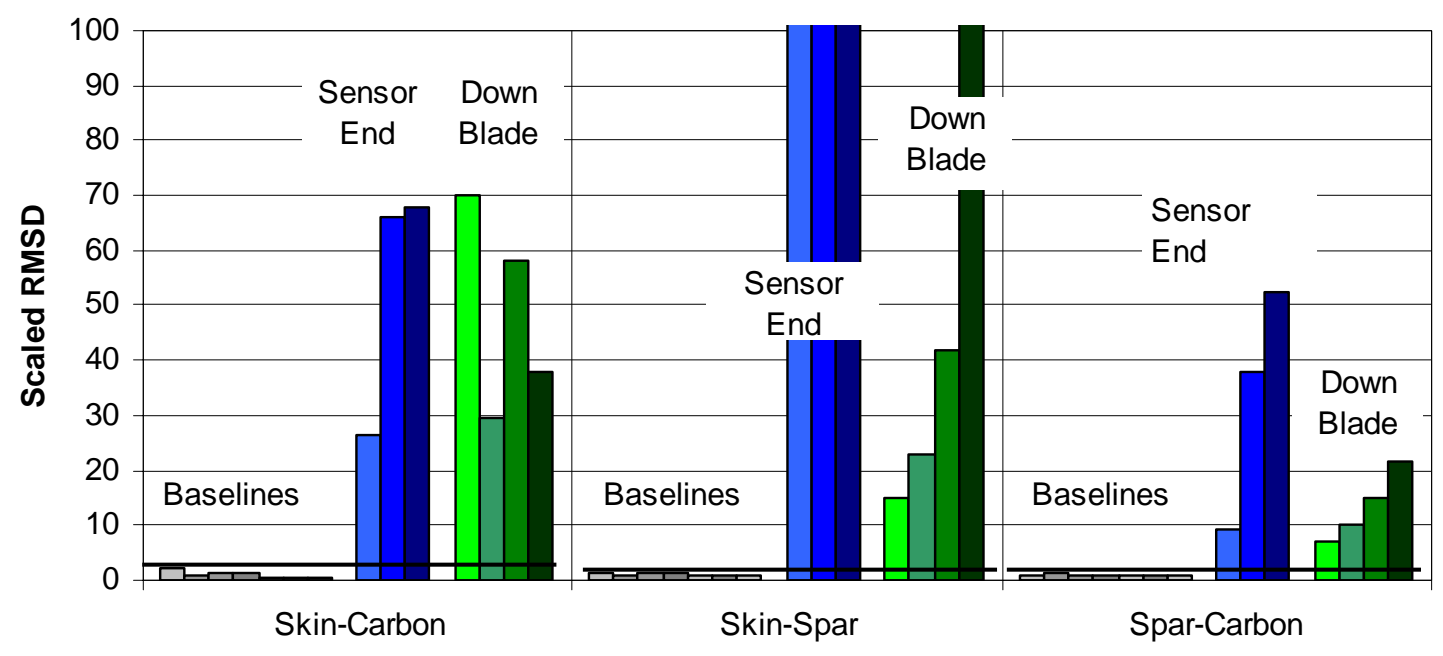

Figure 3.10: Damage metrics for location 1 actual damage HFRF test

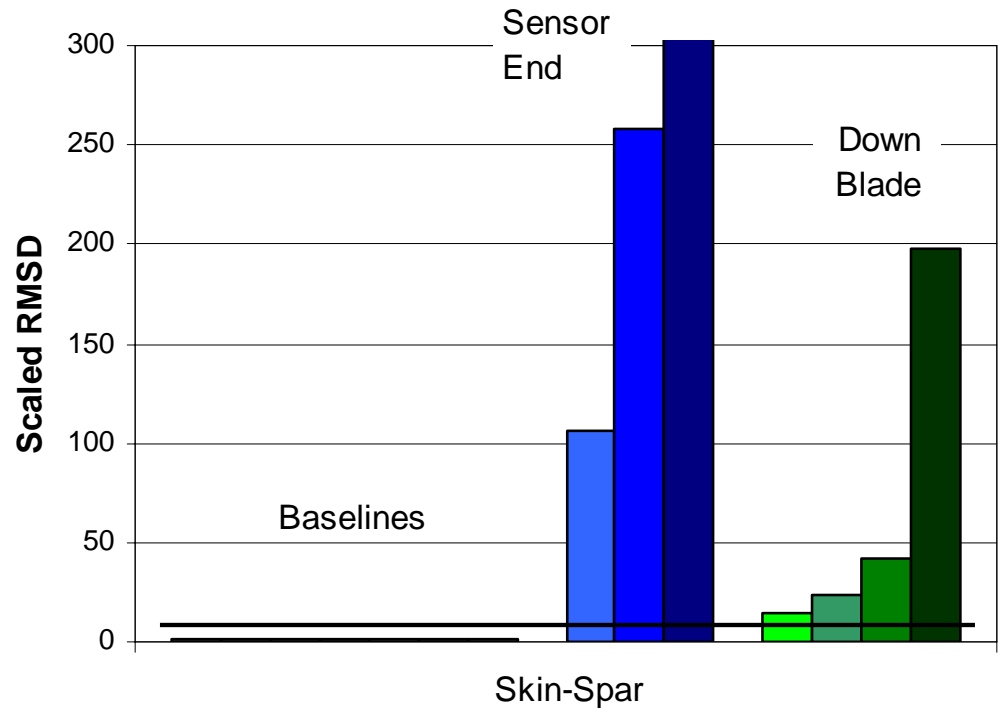

Figure 3.11: Damage metrics for location 1 actual damage HFRF test, enhanced view of skin/spar PZT combination

The skin/spar and spar/carbon PZT combinations were able to effectively sense damage at the sensor end of the blade. As the actual damage in the blade increased, the damage metric also increased. Also for the testing down the length of the blade these combinations were able to effectively sense the damage all the way to $31 \mathrm{~cm}$ down the blade and a clear upward trend is seen in the metrics as the damage nears the sensor. The skin/carbon combination was effective at the sensing end, but the results for the down the 
blade tests are not consistent, as there is no clear trend in damage metric as the damage moves down the blade.

\subsubsection{Location 3 results}

This section contains the results of the HFRF method detecting damage of holes in the adhesive in location 3. Figure 3.12 shows the scaled RMSD damage metrics of these tests for both progressive damage at the sensor end of the blade and damage down the length of the blade for all three PZT combinations. For each PZT combination, the first group is baselines, second is progressively larger holes in the adhesive at the sensor end (darker shade indicates worse damage), and the third is damage down the length of the blade $(28,20,13,5$, and $0 \mathrm{~cm}$ from the sensors, darker shade indicates damage closer to sensor).

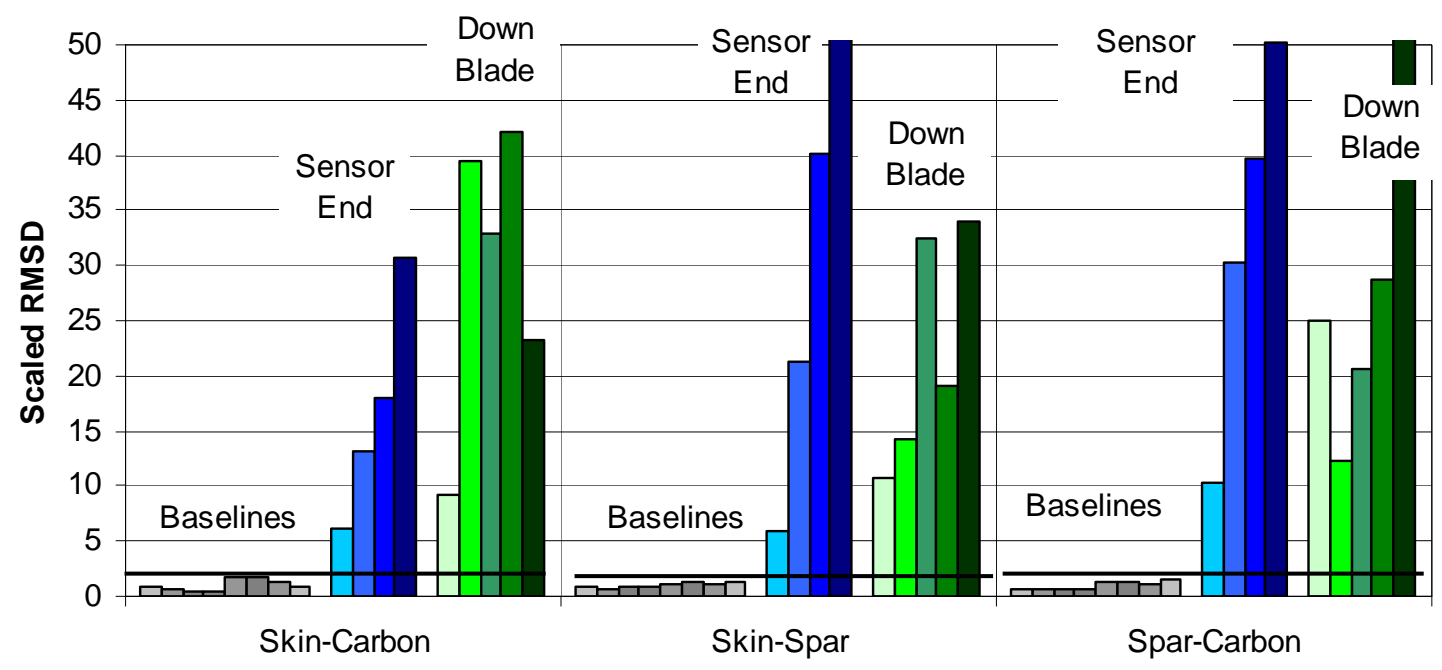

Figure 3.12: Damage metrics for location 3 actual damage HFRF test

All PZT combinations were able to effectively sense damage at the sensor end of the blade and had an increasing trend as the actual damage in the blade increased. However, the down blade results are peculiar in that they for the most part have no trend but do detect damage. 


\subsubsection{Actual damage conclusions}

Based on the results of the actual damage testing for the HFRF method, all of the PZT combinations were able to sense damage on the sensor end of the blade and for the most part had an increasing trend with increasing damage severity. For the damage down the length of the blade, a clear sensing range is hard to determine as damage seemed to be sensed in most all cases, but for the most part no trend was observed as the damage moved down the blade.

\subsection{Conclusions}

The HFRF method was used to detect damage on the same CX-100 WTB section used for IBSHM testing from Chapter 2. Like the IBSHM testing, indirect and actual forms of damage were used to test HFRF on the blade. The HFRF method was able to detect indirect and actual damage with all three sensor combinations. In general it appears that HFRF does have a somewhat higher sensitivity than the IBSHM method. Although, the results for HFRF are not as consistent as those from IBSHM, due to strange results down the length of the blade for HFRF. This is likely due to the HFRF method being somewhat more global in nature. Also, HFRF would be expected to have a somewhat higher sensitivity given the 5 volt excitation for the indirect damage and the 3.5 volt excitation for the actual damage are higher than that used for IBSHM (1 volt). However, IBSHM does produce comparable results to HFRF method and it does so with less excitation and only one PZT patch location. Additionally, using a smaller excitation signal is preferred to reduce power consumption and as discussed in Chapter 1, the hardware for the impedance method can readily be reduced to a small size.

As mentioned in the introduction and in Chapter 1, a method similar to this was used during the fatigue testing of a CX-100 and a similar blade, 3X-100 (Zayas et al. 2007). This method was again used on the fatigue test of a WTB, the TX-100, this time with a frequency range closer to the HFRF method. IBSHM was also used during the same fatigue test and this is the work presented in Chapter 4. When the results of the 
HFRF method for the TX-100 fatigue test are available, a more fair comparison of the two methods can be made as they will have been tested on the same structure during the same test. 


\section{CHAPTER 4 Impedance-Based Structural Health Monitoring of TX-100 Wind Turbine Blade Fatigue Test}

\subsection{Introduction}

Based on the results of the IBSHM tests on the CX-100 WTB section presented in Chapter 2 and Pitchford et al. (2007), SNL wanted to test the impedance method during the fatigue test of the TX-100 experimental WTB. The fatigue test took place at NREL's NWTC just outside of Boulder, Colorado starting in July 2007. While the fatigue test was conducted primarily to determine the structural properties, fatigue strength, and failure mode of the blade, it also aimed to investigate various SHM methods on the blade including IBSHM.

The TX-100 is part of the same SNL/NREL research program as the CX-100 discussed in Chapter 2 that investigates using carbon fiber in subscale experimental blades. The blades are built by TPI Composites and then tested with results compared to modeling. The TX-100, like the CX-100, is 9 meters long and the blades' outer geometries, shown previously in Figure 2.2, are very similar to one another. The interior of the blades are where their designs differ significantly. Carbon fiber is incorporated in the CX-100 in the spar cap regions of the HP and LP skins. The TX-100 has traditional fiberglass spar caps but incorporates carbon fiber into the outer portion of the blade skins with a $20^{\circ}$ bias angle, as seen in Figure 4.1, which results in twist/bend coupling of the blade (Paquette et al. 2006). Coupling the bending and twist modes of the blade has been shown to reduce the blades gust-induced and fatigue loads (Locke et al. 2003). 


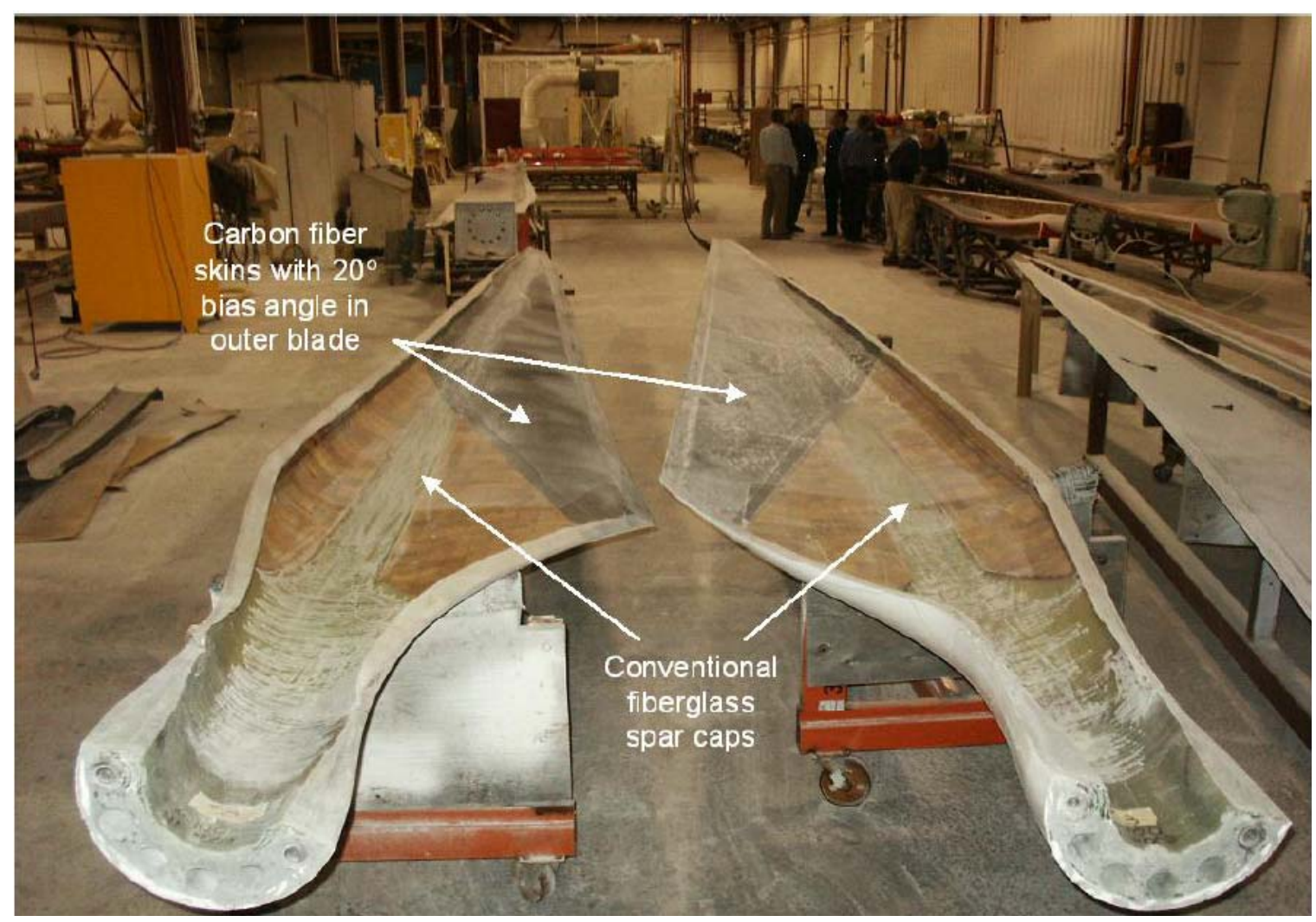

Figure 4.1: TX-100 HP and LP skins showing implementation of carbon fiber into design. (Paquette et al. 2006)

The fatigue test of the TX-100 took place in building A60 at NREL's NWTC. Setup of damage detection instrumentation for the test began July 16, 2007 and the fatigue test started on July 20. The test setup can be seen in Figure 4.2. The root of the blade is mounted to a fixed test stand and then two saddles are attached to the blade. The smaller outboard saddle is attached to the blade at 6.75 meters from the root and is just added weight. The larger inboard saddle is attached at 1.6 meters and has hydraulic actuation to dynamically displace masses. These masses are driven at the first bending frequency to excite the blade to resonance. A closer view of the inboard saddle used in this system can be seen in Figure 4.3. This actuation system is known as the Universal Resonance Exciter (UREX) and this is the first time it has been used. NWTC has previously fatigue tested many wind turbine blade designs including the CX-100, but a different actuation system was used. These previous tests simply utilized a hydraulic actuator attached near the end of the blade to force the blade up and down. 


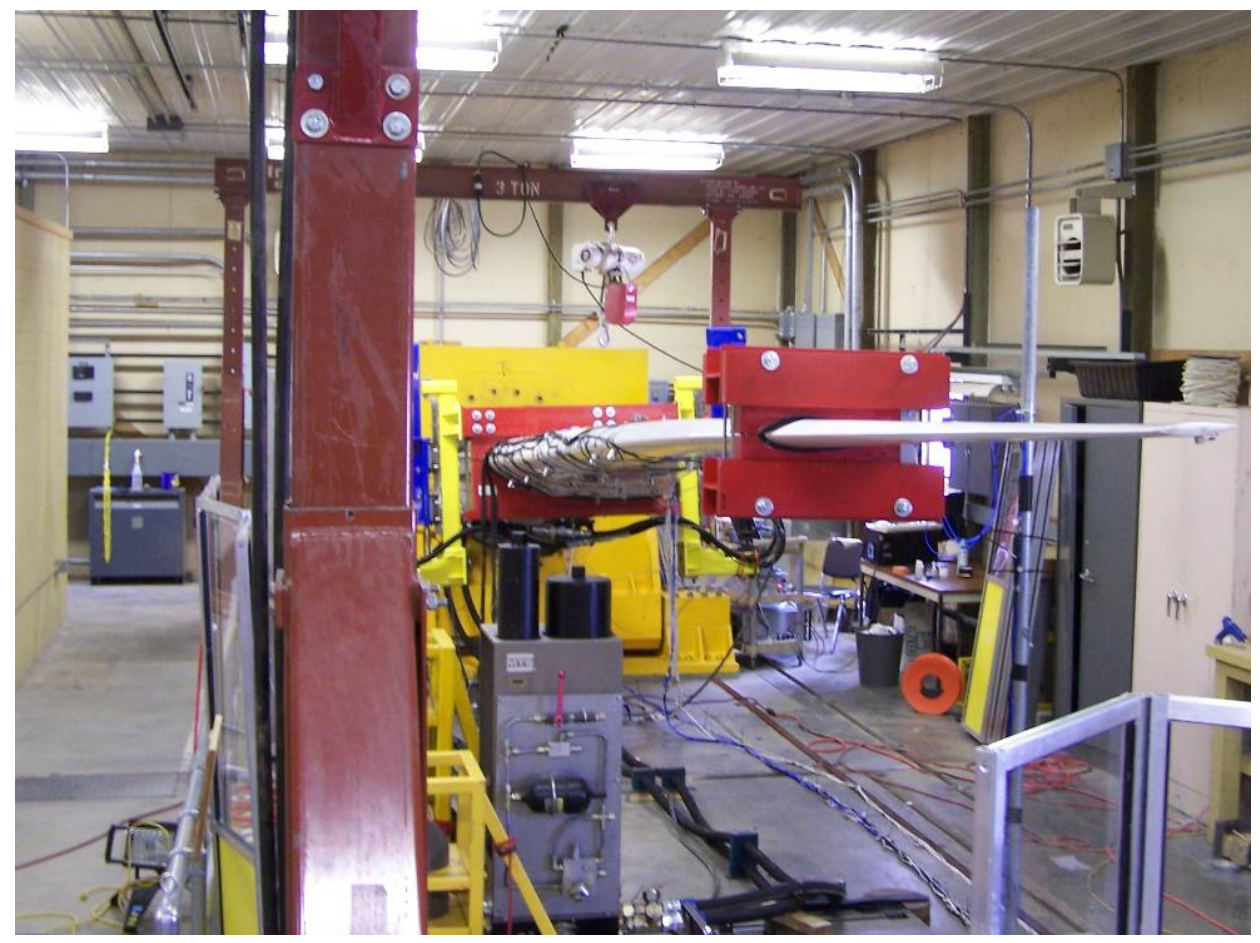

Figure 4.2: TX-100 fatigue test setup

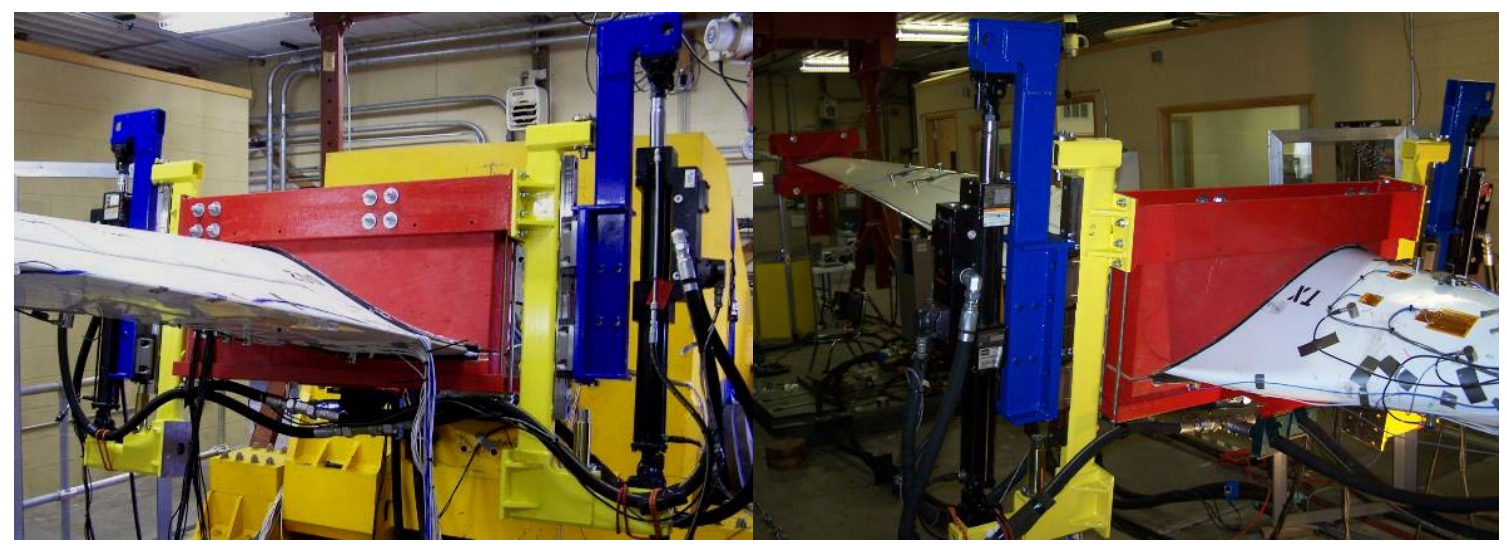

Figure 4.3: UREX inboard saddle

For the fatigue test the blade was driven around $1.3 \mathrm{~Hz}$, its first bending frequency with the added mass of the saddles. The LP side of the blade faced downwards in the test and was kept in constant compression. The load level for the test corresponded to $31 \%$ of the load that caused failure in the TX-100 static test and this was predicted to cause failure in 4 million cycles. However, to speed up the test, after 1 million cycles and for 
every 500,000 cycles after, the load level was increased. The blade was expected to fail between 1 and 3 meters from the root on the compression side. This was just a prediction based on previous tests and models, as one of the main purposes of this test was to find the actual failure mechanism and location. Thirty strain gauges were mounted to the blade in critical locations as were 4 photoelastic panels (Paquette et al. 2007b). The other instrumentation on the blade was for the damage detection methods. In addition to IBSHM, the other methods included on the fatigue test were AE provided by Al Beattie of Physical Acoustics Corporation (PAC), the transfer function method using MFCs by Rudolph Werlink of NASA KSC, and the method of virtual forces investigated by Jonathan White of Purdue University.

As discussed in Chapter 1, AE monitoring has been previously used to detect damage on both static and fatigue blade testing. Approximately 20 PAC AE sensors were mounted to key areas of the TX-100 for the fatigue test to determine when and where AEs occurred during the test (Paquette et al. 2007b). AE is becoming very common for use during blade testing but is not expected to be a method for use on infield blades.

The transfer function method used by Werlink of NASA KDC is the same method discussed in Chapter 1, which was used during the fatigue tests of the CX-100 and 3X100 blades, and is similar to the HFRF method used in Chapter 3. For the TX-100 test, 7 MFCs were used, $257 \times 86 \mathrm{~mm}$ actuators and $538 \times 25 \mathrm{~mm}$ sensors, the same sizes as the previous tests. For this test, a frequency range higher than the previously tested 2-2500 $\mathrm{Hz}$ was used and a filter was added to the setup to allow data to be taken during blade fatigue test operation.

The method of virtual forces considers damage to be theoretical forces acting on an original, healthy structure. Analytically the virtual forces that represent damage can be determined through modal analyses of the healthy and damaged structure with the damaged modal analysis sometimes being of a lesser order than the baseline analysis. This method has been shown to be effective for detecting damage on metallic honeycomb 
thermal protection systems (White et al. 2006) and for these structures the frequency range used for determining damage was typically less than $20 \mathrm{kHz}$ (White 2006). For the TX-100 fatigue test, various types of actuators and accelerometers in approximately 30 locations were mounted to the blade to test the method of virtual forces.

For IBSHM of the TX-100 fatigue test, it was desired to use MFCs as the selfsensing actuators. This was due to there added durability, as PZTs may not survive the life of the fatigue test. In addition, MFCs have been shown to be successful for the impedance method (Park et al. 2007a; Sodano 2003) and for another method of SHM on wind turbine blade fatigue tests (Zayas et al. 2007), as discussed in Chapter 1. MFCs from Smart Material Corporation were used, model M2814-P1 and pictured in Figure 4.4. These MFCs have 28x14 mm active areas and are 37x17 mm overall. As opposed to a traditional impedance analyzer, it was also desired to use Analog Devices AD5933 impedance chip and evaluation board for the impedance measurements. A picture of this device is shown in Figure 4.5. As discussed in Chapter 1, this device has been used by researchers for making impedance measurements for IBSHM. It was advantageous to use this device both because it was easier to get to NWTC and because it is a more suitable device for a system that could actually be implemented inside of an actual WTB. However, as discussed in the next section, due to results of preliminary tests with this device on the CX-100 WTB section, a traditional impedance analyzer was used for the fatigue test in place of the AD5933.

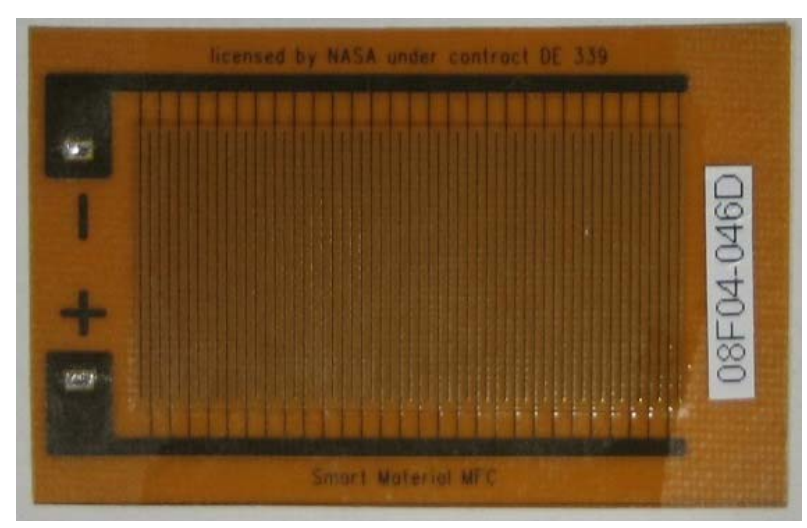

Figure 4.4: Smart Material MFC, model number M2814 P1, 28x14mm active area, 37x17mm overall 


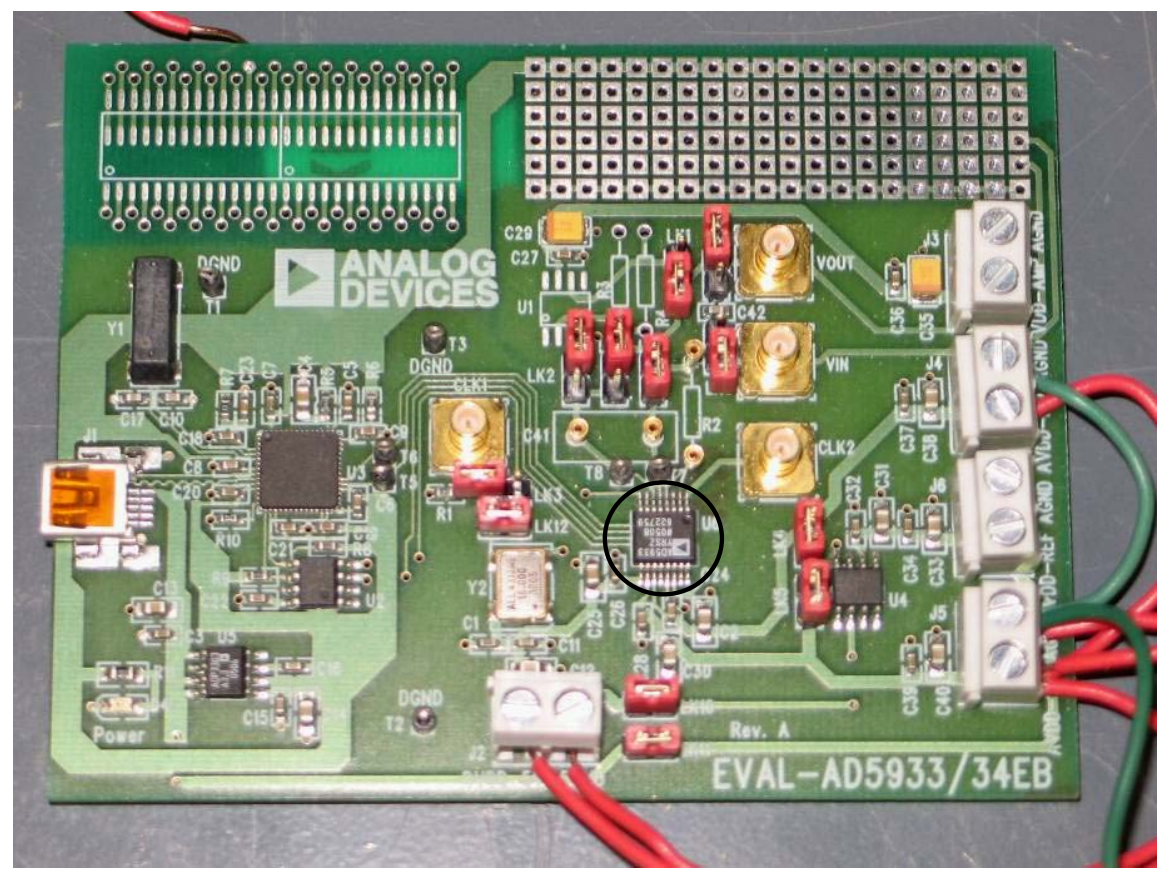

Figure 4.5: Analog Devices AD5933 impedance chip and evaluation board. Evaluation board 100x80mm and impedance chip (circled) $5 \times 5 \mathrm{~mm}$.

\subsection{Preliminary tests on CX-100 wind turbine blade section}

The CX-100 WTB section used for testing in Chapters 2 and 3 was utilized to run some preliminary tests to prepare for the fatigue test of the TX-100. Only one end of the WTB was used for previous tests, so the other end of the section was used for these tests. Four Smart Material M2814-P1 MFCs, the same to be used for the fatigue test, were mounted to the unused end of the WTB section for these tests. These MFCs can be seen in Figure 4.6. The locations of these MFCs were chosen based on the results of IBSHM test results from Chapter 2 which found that the best self-sensing actuator location was the spar. Then MFCs were mounted at this location both on the inside of the blade and the outside, because for the TX-100 fatigue test MFCs would have to be mounted on the outside of the already manufactured blade. These MFCs were used to analyze baseline impedance signatures of the blade, for brief damage detection testing, to test the Analog Devices impedance board, and to compare the HP4194A and HP4192A impedance analyzers. 


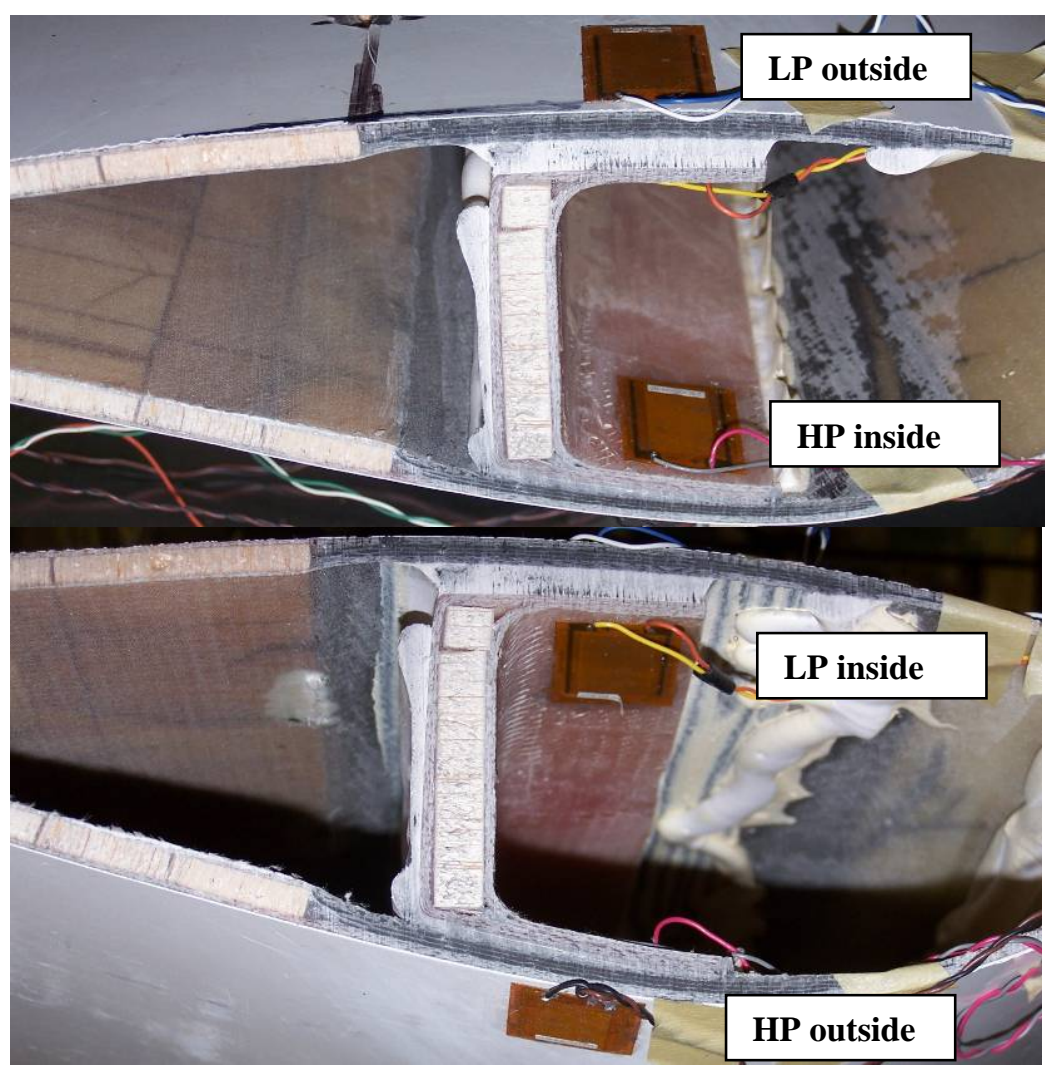

Figure 4.6: MFC locations on CX-100 WTB section

\subsubsection{Baselines}

Baseline impedance signatures were measured for all four MFCs on the CX-100 WTB section from 1-100 kHz. These were obtained with the HP4194A impedance analyzer with a $10 \mathrm{~Hz}$ resolution. The MFC baselines can be seen in Figure 4.7 and Figure 4.8 for the LP and HP sides of the blade, respectively. Compared to the PZT baselines presented in Chapter 2, less structural information is contained in the MFC baselines due to the fewer number of peaks. Also comparing the inner and outer MFCs, the inner MFCs also have more peaks. The frequency range of $10-30 \mathrm{kHz}$, the same range found to be the best based on the results from Chapter 2, is the only range for the MFCs with an acceptable number of peaks for IBSHM. 

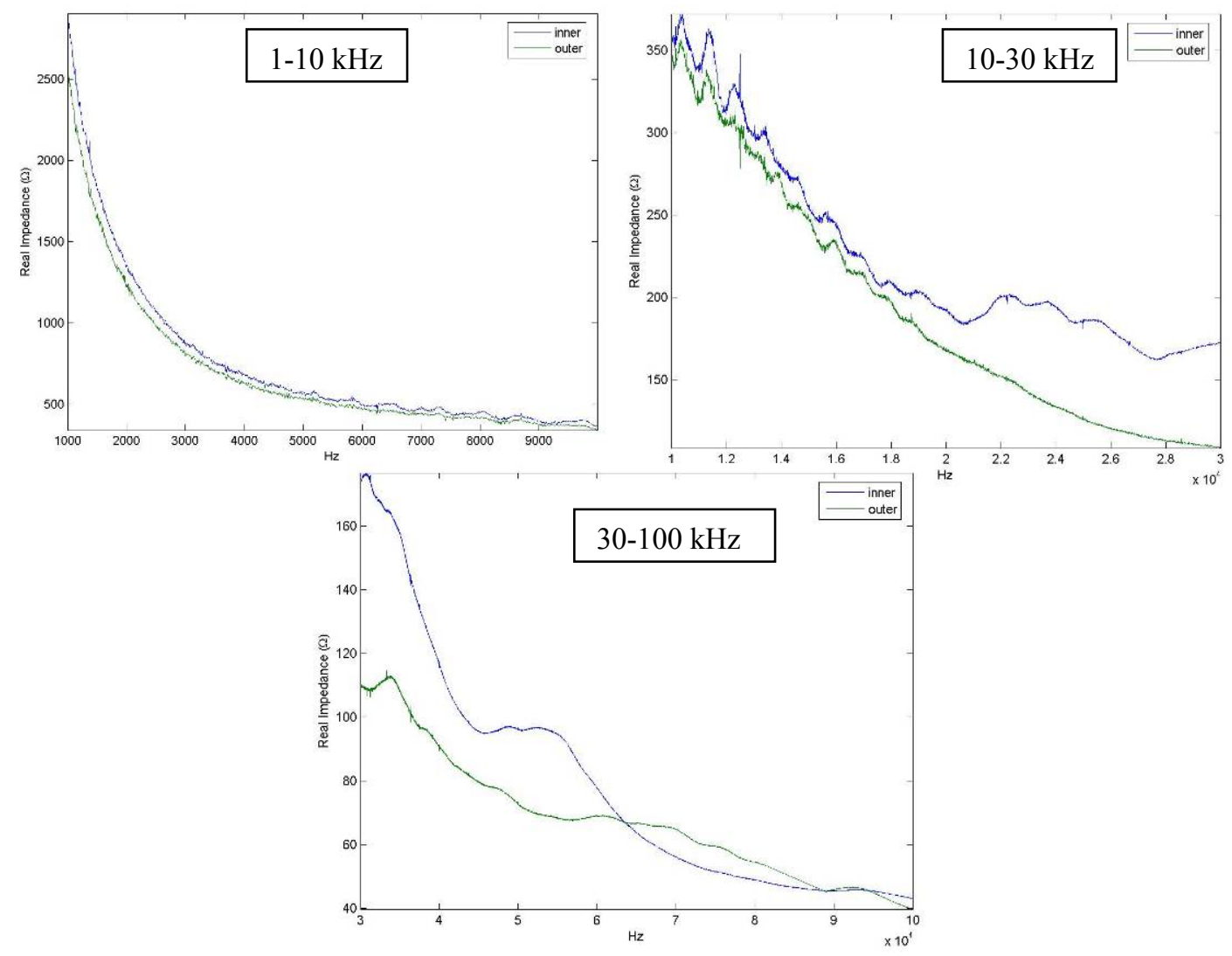

Figure 4.7: Baseline impedance for inner and outer LP MFC
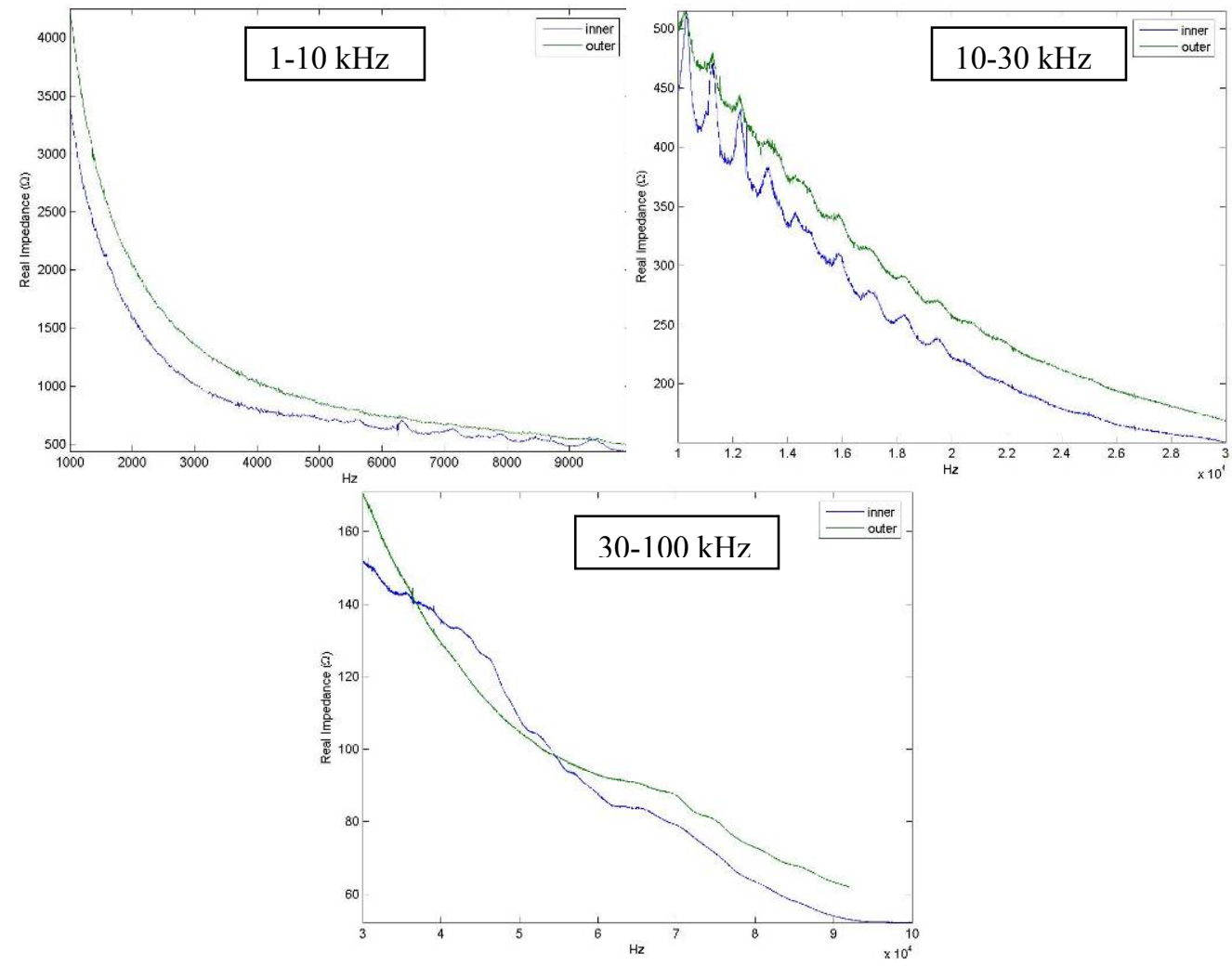

Figure 4.8: Baseline impedance for inner and outer HP MFC 


\subsubsection{MFC actual damage test}

A test with actual damage was carried out to determine if damage could be detected using IBSHM with the MFCs. The MFCs on the LP side of the blade were used in a test identical to the location 3 sensor end tests described in section 2.2.3 where progressively larger holes were drilled in the adhesive between the spar cap and the spar. The HP4194A was used for these tests with a $10 \mathrm{~Hz}$ resolution and a frequency range of 10-30 kHz. The impedance signatures are shown in Figure 4.9 and Figure 4.10 for the inner and outer MFCs, respectively. Change from baseline to damage impedances can clearly be seen, although it is not as great of a change as with the PZT tests from Chapter 2. The damage metrics are shown in Figure 4.11, and similarly, while damage is being detected the results are not as sensitive as where the PZTs results.

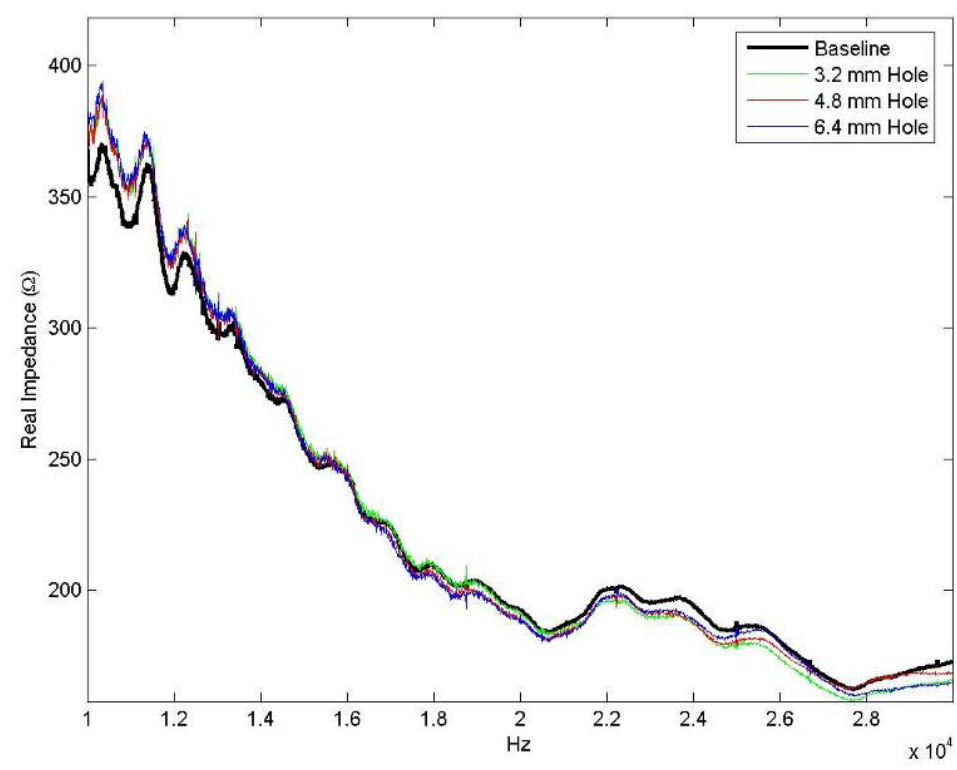

Figure 4.9: Impedance plot for LP inner MFC, progressively larger holes in adhesive 


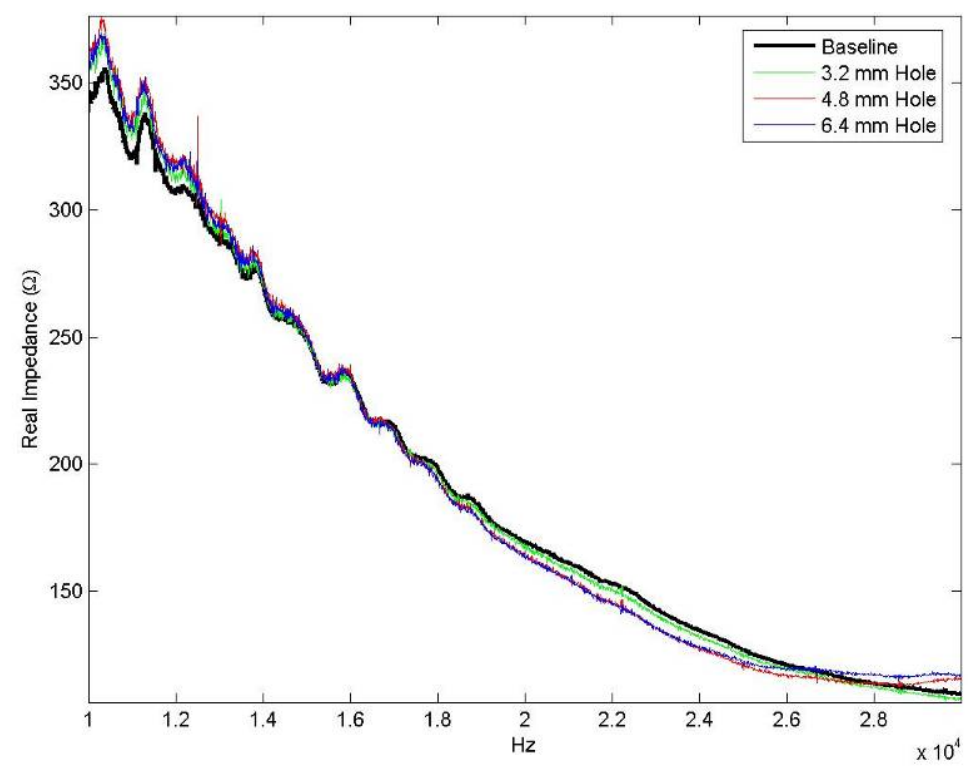

Figure 4.10: Impedance plot for LP outer MFC, progressively larger holes in adhesive

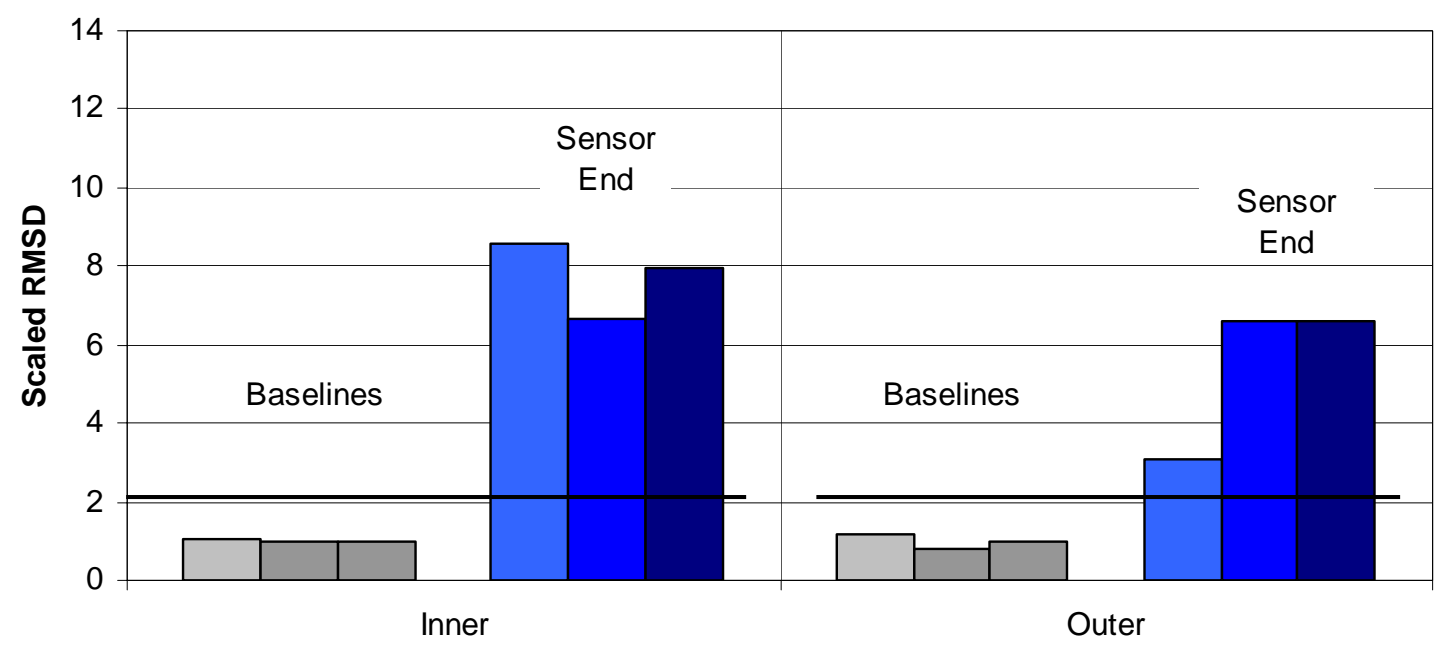

Figure 4.11: Damage metrics for LP inner and outer MFC, progressively larger holes in adhesive

\subsubsection{Analog Devices AD5933}

The Analog Devices AD5933 impedance chip and evaluation board were used to measure the baselines of the 4 MFCs on the CX-100 WTB section. The evaluation software provided by Analog Devices with the board was used to interface between a laptop and the board. For the baseline impedances, the frequency range used was 10-30 
$\mathrm{kHz}$. The maximum number of increments possible was used (511) for a frequency resolution of $39 \mathrm{~Hz}$. Also, the maximum number of settling time cycles (511) were used with a 1 volt excitation. An $8 \mathrm{k} \Omega$ resistor was used as the feedback resistor for the AD5933, as this was approximately the median value of impedance for the MFCs in the 10-30 kHz range. The impedance measured by the AD5933 is shown in Figure 4.12, broken into magnitude and phase. The same things measured with the HP4194A are shown in Figure 4.15 for comparison. From the results, the AD5933 was able to accurately measure the magnitude of the impedance, but not the phase. Therefore calculating the real part of the impedance that is typically used for the impedance method would not be useful. This same issue was noted by Peairs when he used the impedance method and an AD5933 on a railroad track section (2006). In the case of the railroad track, enough structural information was present in the magnitude of the impedance to simply use the magnitude for SHM calculation. However, this is clearly not possible with the WTB section as all of the structural information appears in the phase of the impedance as can be seen in the measurements with the HP4194A in Figure 4.13.
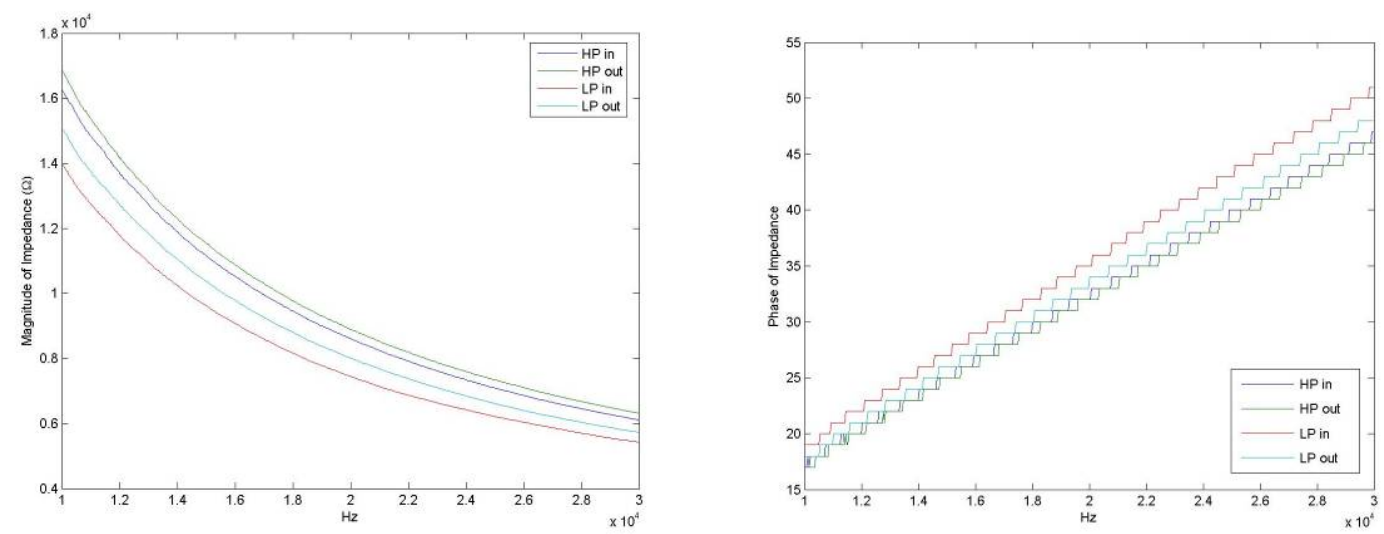

Figure 4.12: Magnitude and phase of baseline impedance for 4 MFCs: AD5933. 

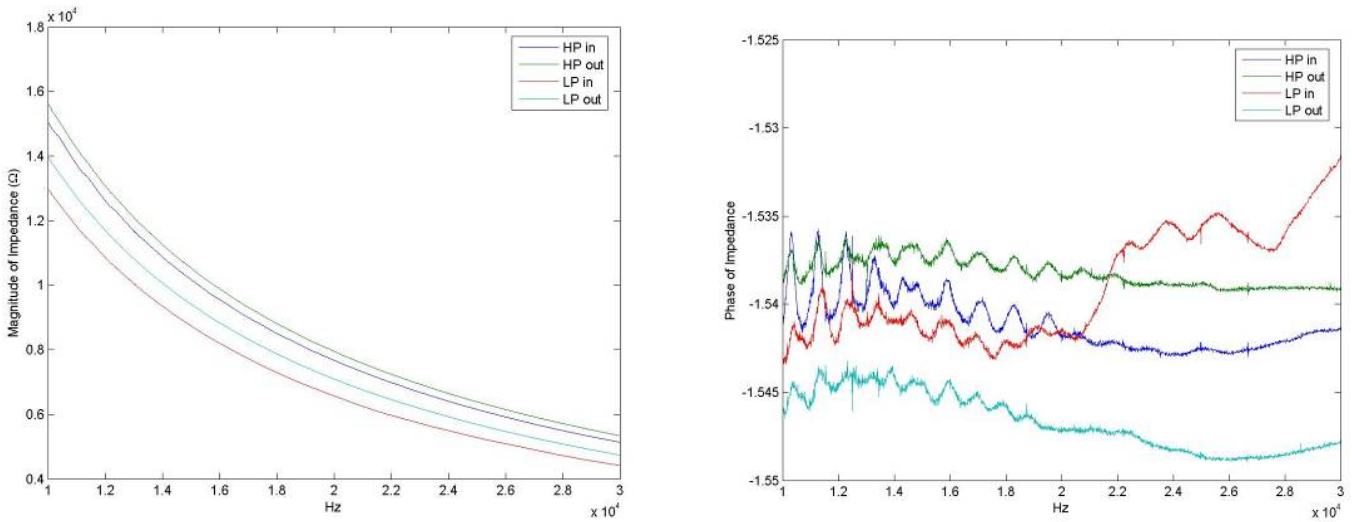

Figure 4.13: Magnitude and phase of baseline impedance for 4 MFCs: HP4194A.

The problem with the AD5933 is with the way it breaks its impedance results into complex parts. A method to solve this problem and obtain functional results for real impedance from the AD5933 has been devised by Overlay et al. (2007). This method was attempted to solve the problem with the results obtained from the WTB, but without success. It is likely that the AD5933 could measure the real impedance of the MFCs on the WTB, however a method was needed to measure impedance for the fatigue test that was sure to produce reliable results; therefore, an impedance analyzer was taken to NWTC.

\subsubsection{HP4192A compared to HP4194A}

The HP4194A that had been used for previous tests was not available to be used for the fatigue test, which was likely to last for one to two months, so a HP4192A that was available was used instead. This impedance analyzer is an older model and takes significantly longer to take readings than the HP4194A that have the same frequency resolution. In order to take measurements in a timely manner during the fatigue test, a lower frequency resolution would have to be used than had been used in previous tests. A brief indirect damage test was run on the blade with the MFCs and both impedance analyzers to determine if damage could be detected with less frequency resolution. Three baseline impedances were measured and then a clamp was attached to the spar and damaged impedance was measured for all 4 MFCs and with both impedance analyzers. The impedance signatures for these tests can be seen in Appendix E. Change can be seen 
from baseline to damaged state in all cases, and while the HP4194A does have better resolution, the peaks in the impedance as well as change from baseline to damage impedance is captured by the HP4192A. The resulting damage metrics for these tests can be seen in Figure 4.14. The HP4192A is clearly sensing damage and actually has higher damage metrics than the HP4194A. Based on these results, the HP4192A was used for the fatigue test at NWTC.

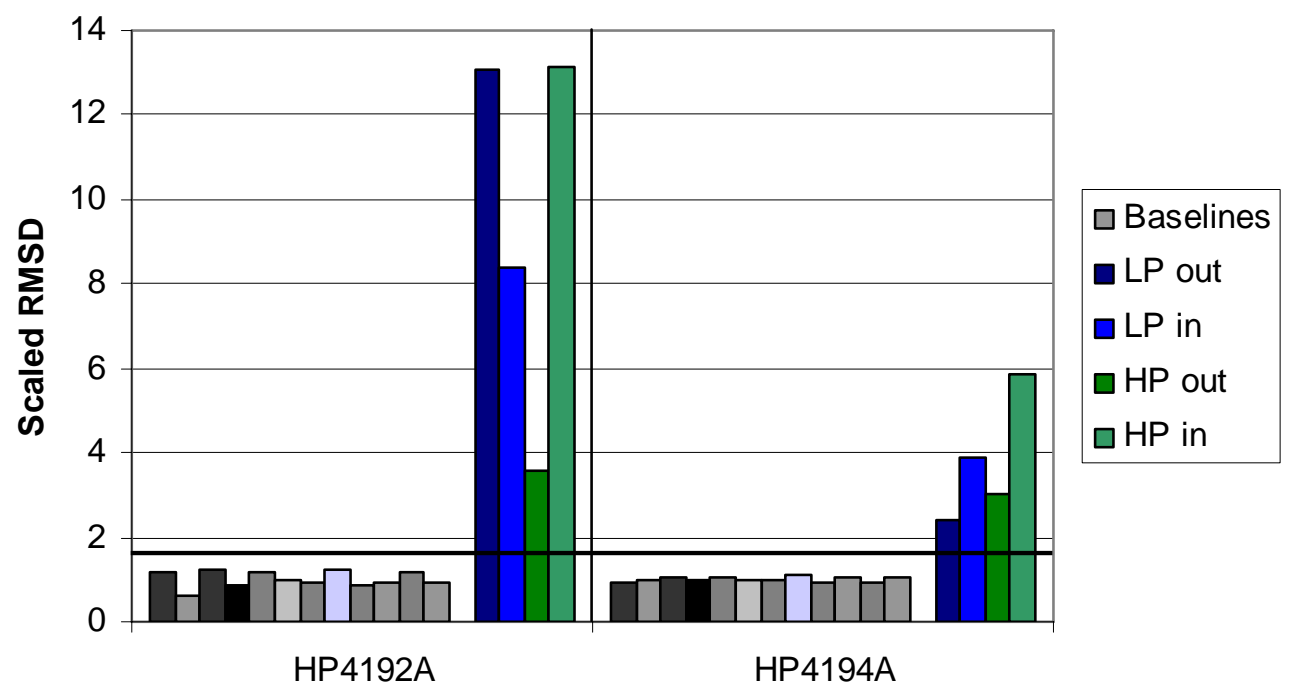

Figure 4.14: Damage metrics comparing HP4194A and HP4192A results of indirect damage test using MFCs

\subsection{Experimental setup}

The HP4192A was shipped to NWTC for the fatigue test along with a laptop running Piezoelectric Resonance Analysis Program (PRAP) version 2.1 software to control the impedance analyzer and record data. This setup can be seen in Figure 4.15. Also seen in this figure and in a closer view in Figure 4.16 is a switchbox. The switchbox was built so that all 6 MFCs that were bonded to the blade could be connected to the impedance analyzer simultaneously, and then one could be selected at a time to be input. This greatly simplified the routine for collecting impedance data. 


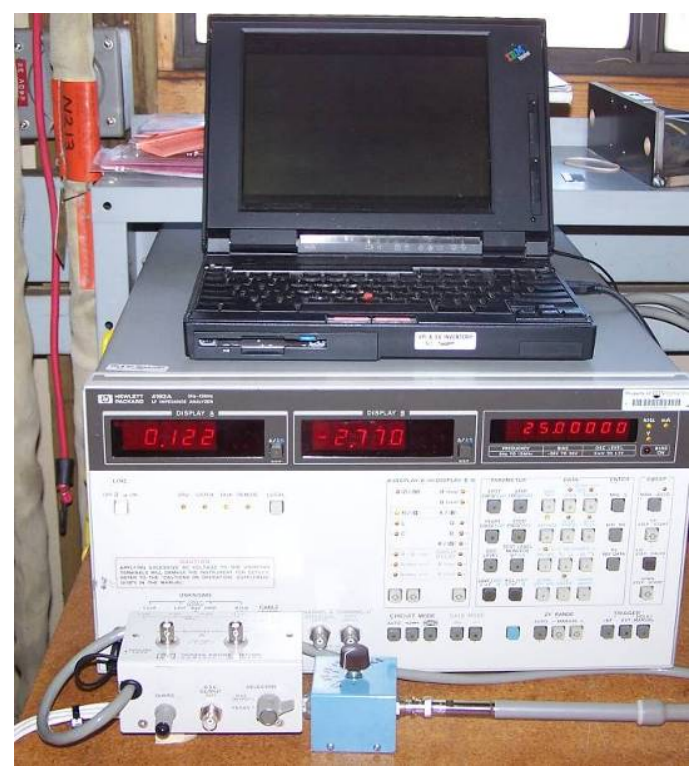

Figure 4.15: IBSHM measurement setup for TX-100 fatigue test. Laptop, HP4192A impedance analyzer and switch box.

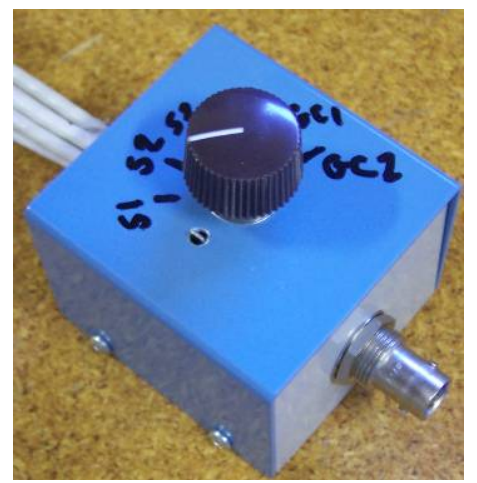

Figure 4.16: Switch box to input 6 MFCs into HP4192A for TX-100 fatigue test.

The self-sensing actuators attached to the blade were 6 Smart Material MFCs, model M2814-P1. The locations where the MFCs were bonded are all on the LP, downward facing side of the blade between 1 and 3 meters from the root. This is the area were failure was expected from modeling and previous similar tests. The locations within this area were chosen based on the internal geometry of the blade. This geometry along with the sensor locations is illustrated in Figure 4.17. Failure was expected near the spar cap close to the 1 meter area, so 3 MFCs were mounted in this area and were named s1, s2, and s3. One additional MFC was mounted on the spar cap region near the intersection of the spar cap, glass/balsa skin, and carbon/balsa skin and was named s4. Finally, 2 MFCs were mounted on the glass/balsa and carbon/balsa intersection on the 
skin, one at about 2 meters, gc1, and one near the spar cap, gc2. These MFCs were bonded using the same procedure used by Rudolph Werlink of NASA KSC to bond the MFCs for the transfer function method both for the TX-100 fatigue test and the previous tests. This procedure is similar to strain gauge bonding and uses the same materials. Figure 4.18 shows a picture of the MFCs bonded to the WTB, along with some of the other sensors used on the blade. The sensors outlined in green are for AE, those outlined in blue are accelerometers for the virtual forces method, and the other MFCs outlined in red are from the NASA KSC test. Finally, the gray sensors are the photoelastic panels.

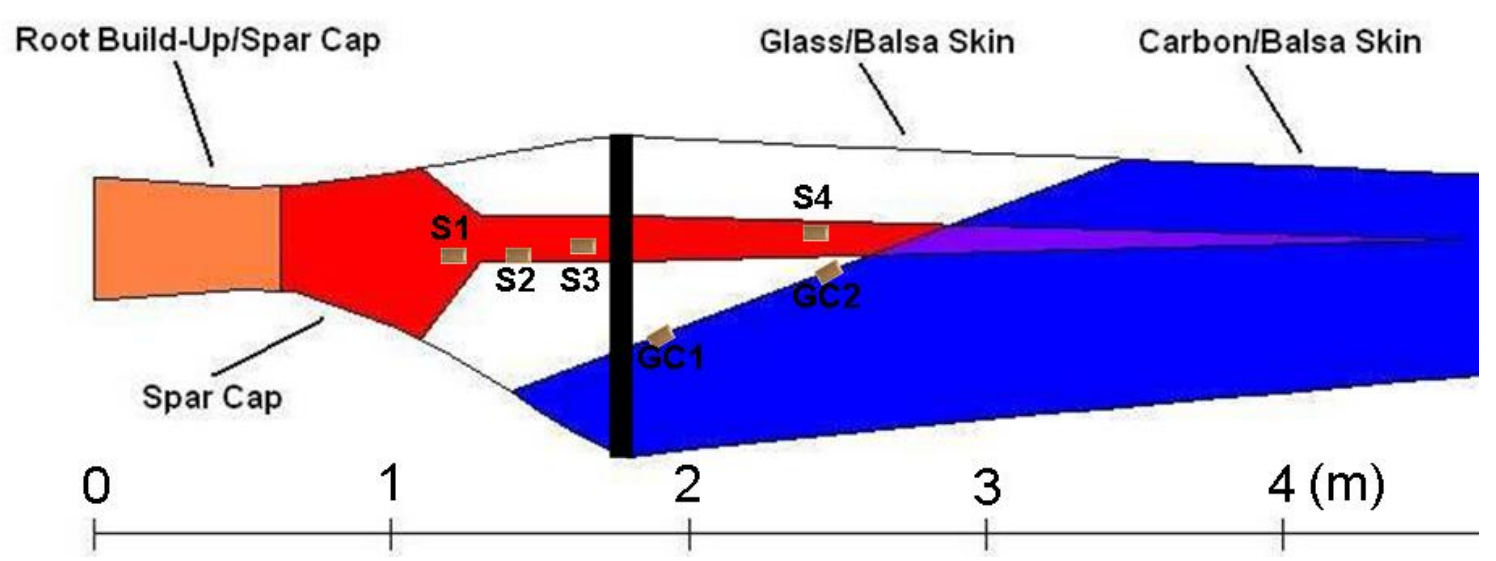

Figure 4.17: Layout of MFCs for IBSHM on TX-100 schematic.

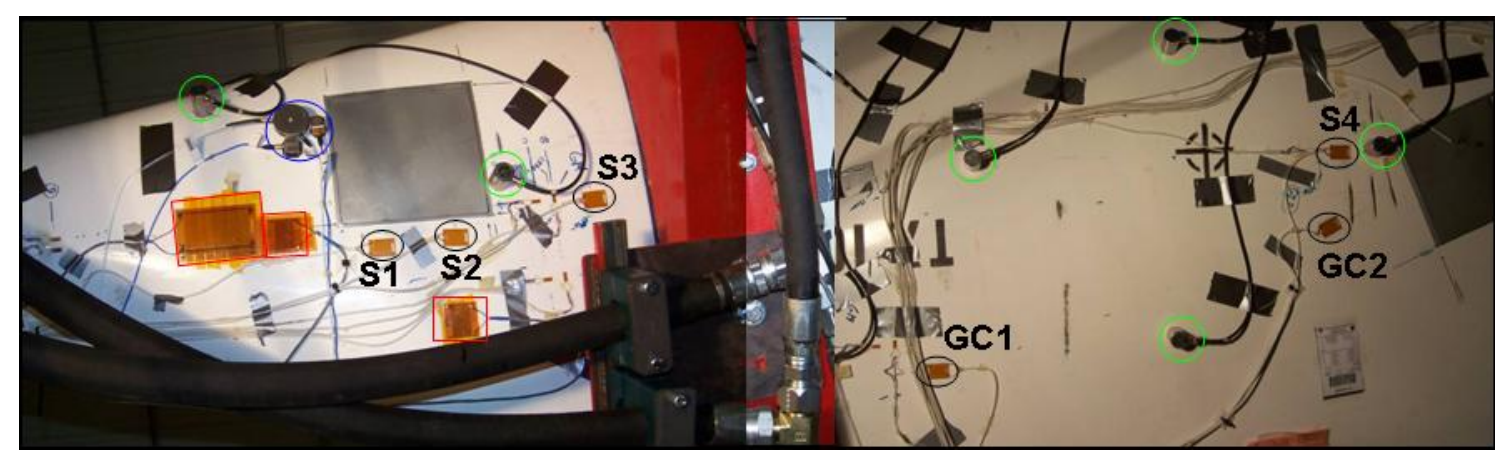

Figure 4.18: Picture of MFC layout on downward facing (LP) side of TX-100. Also shown are sensors for other damage detection methods. 


\subsection{Results}

\subsubsection{Initial results}

Initially, baseline impedance signatures from $5-60 \mathrm{kHz}$ were measured for all 6 MFCs. An example, s3, is shown in Figure 4.19 and all 6 baselines are shown in Appendix F. From these results, very little if any structural information is contained in the impedance signatures as there are practically no peaks in the impedance. This, of course, is a very disappointing result because it is unlikely that with impedance signatures of this nature that any damage could be sensed. However given that the equipment was already in place, testing was continued despite the non-ideal preliminary results with the hope that when damage did occur, it would create enough of a change in the impedance for peaks to appear or for the profile to change. A frequency range of 5-25 kHz was selected based on the baseline measurements.

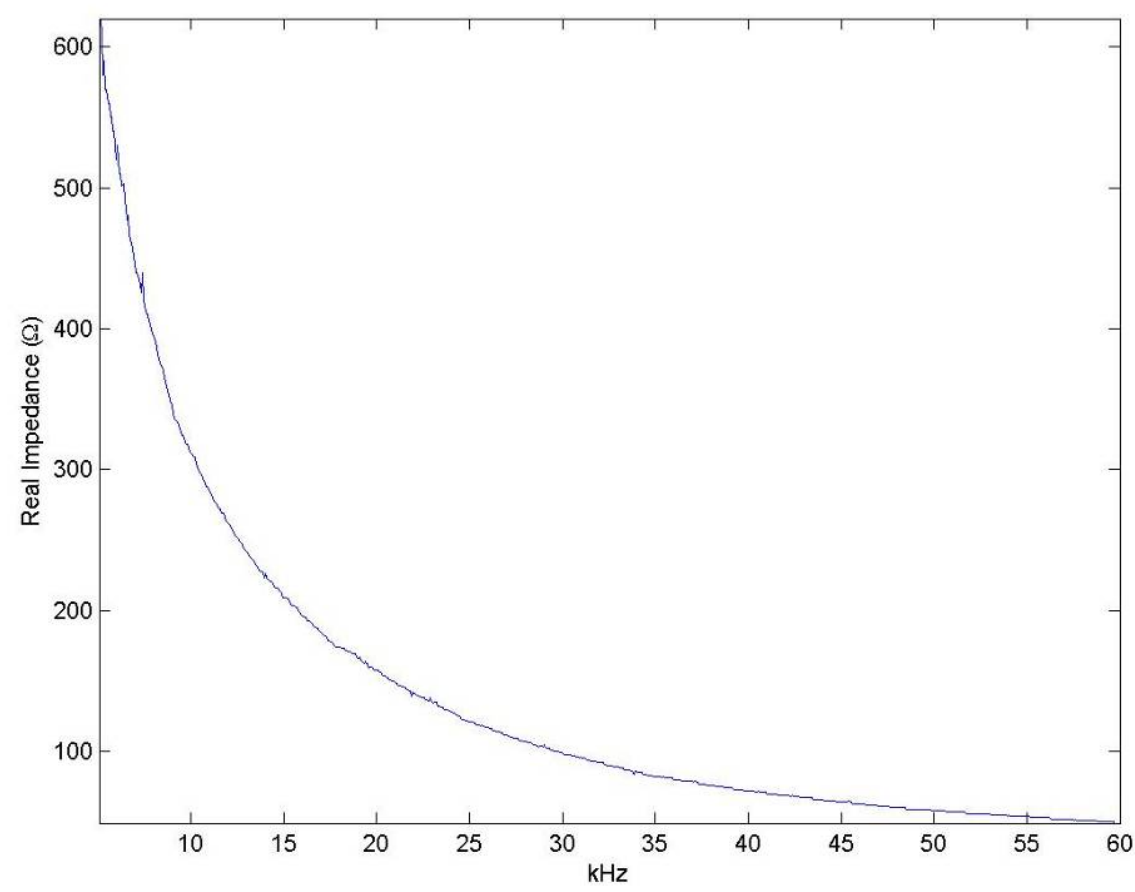

Figure 4.19: Baseline impedance plot for $\mathrm{s} 3 \mathrm{MFC}, 5-60 \mathrm{kHz}$ 


\subsubsection{During fatigue test operation}

During the first day of the fatigue test, data was collected while the blade was being excited. A sample of one of these impedance signatures from $\mathrm{s} 3$ is shown in Figure 4.20. An impedance signature from the stopped blade is also shown for reference. The blades frequency is around $1.3 \mathrm{~Hz}$ and the data is collected from the HP4192A at approximately $0.8 \mathrm{~Hz}$. As can be seen in Figure 4.20, these relatively close frequencies created a "beat" frequency in the impedance data. This could most likely be cured with some type of filter to take away the beat from the data. However, given that the baseline measurements were not ideal, it was decided that one more possible complication to the data would not be worth being able to take data. Additionally, the fatigue test was to be stopped on the order of once a day so data could be taken during this time without problem.

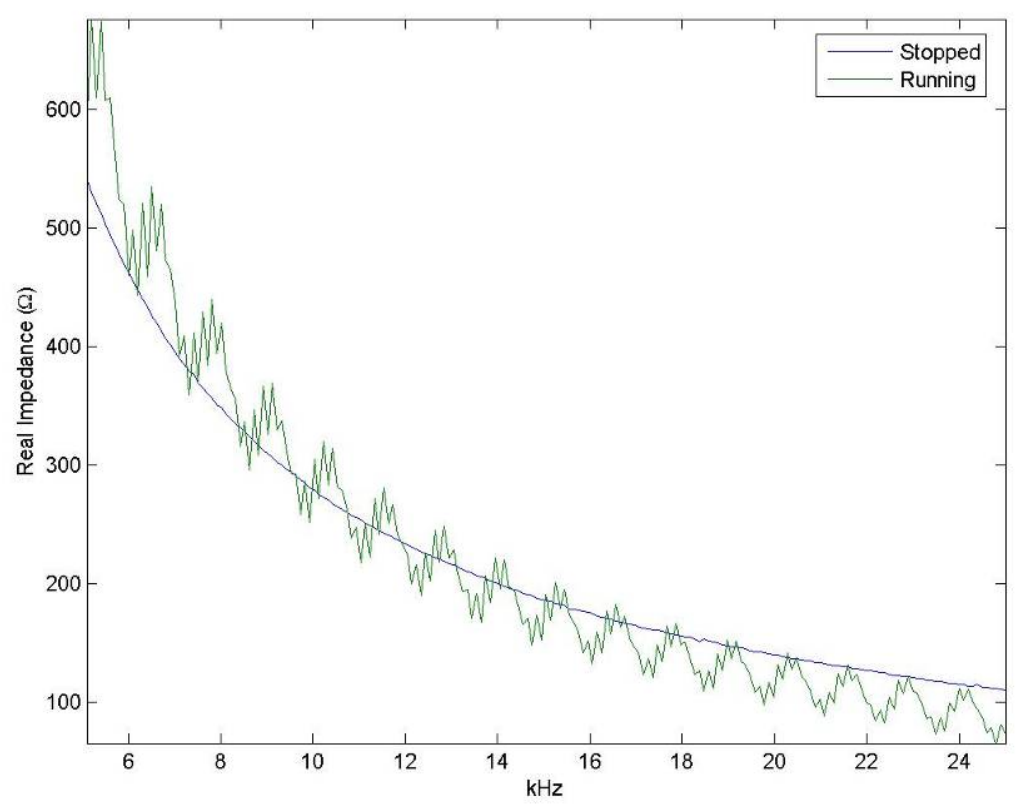

Figure 4.20: Impedance signature of s3 MFC, both with fatigue test stopped and running illustrating "beat" frequency 


\subsubsection{Data collection and baseline damage metrics}

The impedance signatures from the 6 MFCs were recorded roughly every day and occasionally twice a day, mainly whenever the test was shut down to inspect the blade or for maintenance of the system. The impedance measurements for baselines, with the blade running, and the first 4 sets of data during stops in the fatigue tests were recorded by the author. After this, technicians at the NWTC took the measurements following the procedure shown in Appendix G. During the first two weeks of the fatigue test, the test was shut down over nights and weekends. This was because of the use of the new UREX excitation system and as a precaution to monitor the blade closely during the early cycles of the test. For this reason, the blade only accumulated 236 thousand cycles during this time. After this, the blade was run overnight and on weekends and the test accumulated nearly 100 thousand cycles per day. Due to the low number of cycles and because no damage was discovered during the first 236 thousand cycles, it seemed logical to consider measurements from this period of the test as baselines. Also, it was desirable for the baseline to have the variation from the fatigue test being run but before damage was accumulated, as opposed to just taking 10 baseline readings before the test began back to back. The two stages of testing as well as where the baseline measurements were taken from are shown in Figure 4.21.

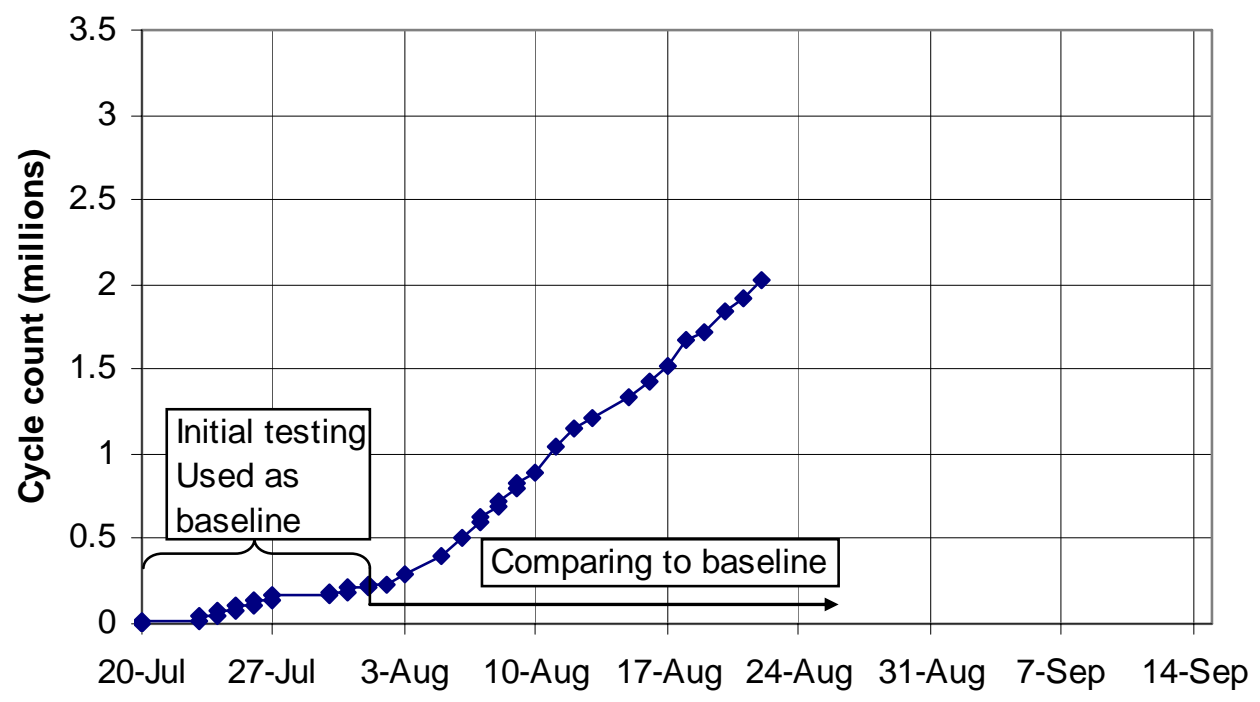

Figure 4.21: Graph of fatigue test cycle count showing where baseline measurements took place. 
Eleven data sets were used to make up the baseline damage metrics. The initial reading before the test began was compared to the next 10 readings to give 10 damage metrics. These metrics were scaled so that their average was equal to one and then a threshold was calculated using the method discussed in section 2.2.1. These metrics for the 6 MFCs are shown in Figure 4.22 and the blue lines represent the thresholds. The average of the 6 thresholds is 1.75 scaled RMSD, and this value will be considered the threshold for damage for all 6 MFCs.

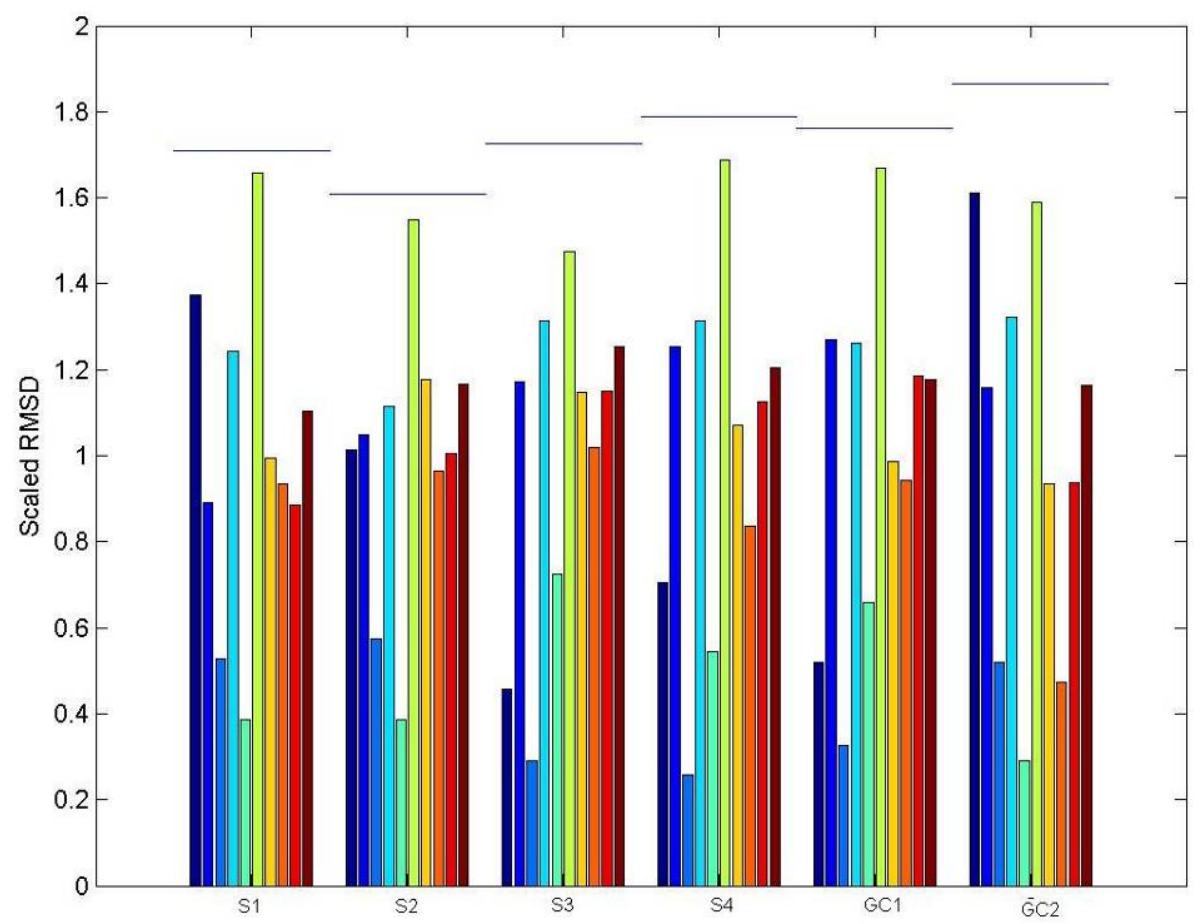

Figure 4.22: Baseline damage metrics for fatigue test for 6 MFCs shown with resulting damage thresholds.

\subsubsection{Damage detection}

After the initial 11 data sets that make up the baseline, the impedance signatures that followed were compared to the initial reading to produce a damage metric and then it was scaled based on the average of the baselines. These metrics were then compared back to the 1.75 damage threshold. These damage metrics can be seen in Figure 4.23 plotted versus the cycle count of the fatigue test and the damage threshold shown as a 
blue line. The impedance signatures that defined these damage metrics can be seen in Appendix H. As can be seen in these results, there was very little change in any of the impedance signatures throughout the fatigue test with the exception of gc2. However, this change was due to issues with the connection of gc2 at the MFC or in the switch box. These results are also illustrated in the damage metrics. All of the damage metrics follow generally the same random trend and stay under the threshold with the exception of gc2. The two points for gc2 that go out of the scale of the figure around 1 to 1.2 million cycles correspond to two bad readings. These two bad readings are obvious in the impedance signatures and appear to be due likely to the bad connection. Other points around 1.7 million cycles appear to possibly be damage, but then the damage metric falls back down below the threshold. Given this sensors previous problems, this is more likely continued problems and not detection of damage.

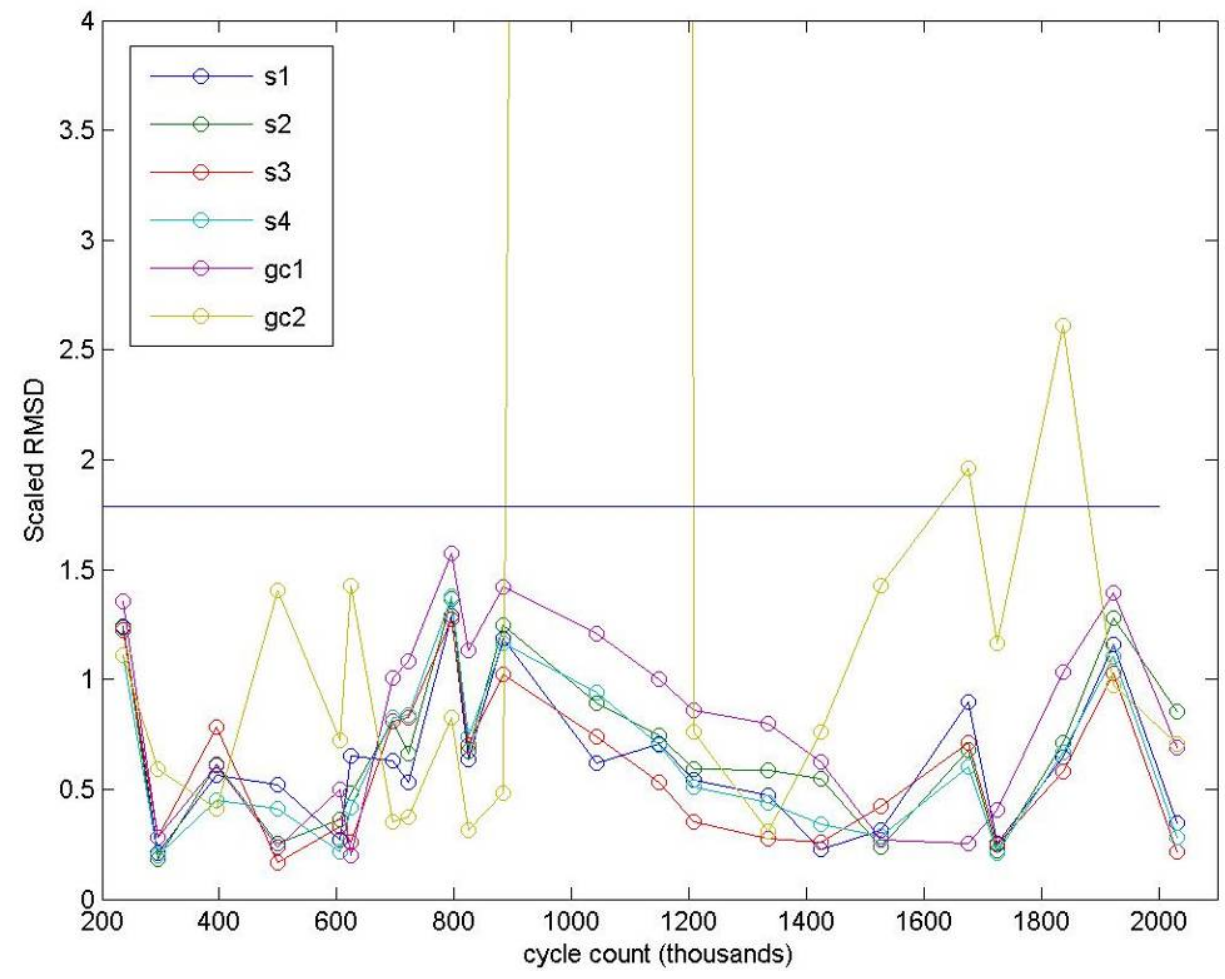

Figure 4.23: Damage metrics for fatigue test plotted versus cycle count and shown with damage threshold. 


\subsection{Conclusions}

The IBSHM method was used to detect damage during the fatigue test of a TX100 WTB at the NWTC. For this test, it was desired to use MFCs for the self sensing actuators and an AD5933 to measure impedance. In addition, for the fatigue tests, the MFCs would have to be mounted on the outside of the blade. Unfortunately, problems arose getting the AD5933 to produce acceptable real impedance results so an HP4192A impedance analyzer was used in its place. Preliminary tests on the CX-100 WTB section showed that using MFCs on the blade in place of PZTs and mounting the MFCs on the outside of the blade instead of the inside caused less structural information to show up in the impedance, however damage was still able to be detected.

For the fatigue test, $6 \mathrm{MFCs}$ were mounted to the blade. Impedance could not be recorded during operation of the fatigue test because it caused a beat frequency to develop in the impedance data. Baseline readings of the 6 MFCs showed no peaks and therefore no structural information from 5-60 kHz. However, the testing continued but as expected from the initial baselines no damage was detected. These results are disappointing considering that IBSHM seemed to show the ability to detect damage on the initial tests on the CX-100 WTB section. However, on the actual blade during the fatigue test the method was insensitive to damage. This is possibly due to the combination of several reasons: using MFCs, mounting on the outside of the blade, and

likely that the full blade was larger, more massive, and not merely a section-and therefore has no boundaries close to the MFCs. 


\section{CHAPTER 5 Summary and Conclusions}

\subsection{Thesis Summary}

Wind energy is a promising, fast-growing power source and SHM of WTBs could potentially help wind energy technology advance to its potential. The impedance method is a SHM technique that has shown promise on a wide variety of structures, including composites, and is readily made autonomous and self-contained. This work was conducted to determine the feasibility of using the impedance method for SHM of WTBs by exploring if IBSHM would be able to detect damage in WTBs.

Chapter 1 introduced this research with a brief background of wind turbines and a look at various methods that have been previously used to detect damage in WTBs. It also presented the basis for IBSHM and an overview of previous research on the method. Chapter 2 contained the initial work exploring IBSHM for WTBs, a series of tests performed on an actual blade section using the impedance method to detect indirect and actual forms of damage. These tests showed that IBSHM could indeed detect damage on the blade section, although the sensing range was fairly low. Still, based on these results the method seemed promising for use in critical blade areas. Chapter 3 presented a series of tests similar to those in Chapter 2. The same blade section and type of tests were used, only using the HFRF method. This method was chosen for comparison due to its success on composite plates and the use of a similar method on WTB fatigue tests. Based on the results, HFRF was comparable to IBSHM with slightly more sensitive but less consistent results.

Finally, in Chapter 4 the impedance method was used during the fatigue test of a TX-100 WTB. For this test, the impedance method was found to not be sensitive to structural changes in the blade. MFCs were used as self-sensing actuators and had to be placed on the outside of the pre-manufactured blade. These factors along with the blade 
being larger than the previously tested section and not having any boundaries near the sensors possibly contributed to insensitivity.

\subsection{Conclusions}

The tests on the CX-100 WTB using the impedance method were successful enough to continue to pursue testing the method on the TX-100 fatigue test. Unfortunately, during the fatigue test, the impedance method was unsuccessful in detecting damage as almost no structural information showed up in the impedance of the MFCs. The probable reasons for this insensitivity are mostly unavoidable. MFCs need to be used instead of PZTs because of their added durability. Obviously, the blade geometry for actual applications will be like the fatigue test and not the blade section. Mounting the MFCs on the inside of the blade was the only option for this fatigue test, although inside mounting could be done during the manufacturing of the blades. However, it is unknown exactly how much this would help. In conclusion, for the given setup of IBSHM of WTB investigated, the method seems to be insensitive to damage and therefore the impedance method is not currently feasible for WTB SHM.

\subsection{Recommendations and future work}

The lack of success of the impedance method on WTB is disappointing; however, blades are an extremely challenging structure for SHM as they are largely made of balsa wood and empty space. This highly damped structure makes a low voltage excitation method like IBSHM rather difficult. One possible solution to increase the sensitivity of the method would be to increase the excitation voltage. This could not be accomplished with a traditional impedance analyzer but would be possible if special hardware was developed. While this would in some ways defeat the purpose of IBSHM as a lowenergy method, since this application is attached to power source in the megawatt range, maybe a small amount of power could be borrowed from the WT. Excitation energies significantly higher than 1 volt would not be that large of an increase compared to the 
overall output of the turbine. In fact the other methods being tested for SHM of WTBs use excitation signals significantly higher than 1 volt.

If a blade section larger than the CX-100 section could be obtained for testing, some of the issues found in the results of this work could possibly be resolved. A larger section could be tested in a lab setting and the results compared to those of the smaller section. Then possibly a better explanation of why the problems arose with the larger blade could be determined. If these issues along with the excitation voltage are resolved enough to increase sensitivity to more acceptable values, more opportunities to test WTBs at NWTC will exist both during future fatigue tests and static tests to failure.

As presented in Chapter 1, the transfer function method used in two previous WTB fatigue tests was somewhat successful detecting damage on one blade test, but on the other the damage metric they were using fluctuated almost randomly, much like the results of the IBSHM for the fatigue test. This challenging structure is difficult for the other forms of SHM as well. When results become available for the other methods used on the TX-100 blade test, and any future tests which are similar, they should be compared to the results of this work.

Current research for IBSHM is mainly focused on making the method selfcontained with wireless communication and power harvesting. These are very important items for advancing the technology to the point where it can be used on real world structures. However, it is also important to take opportunities like the one in this work to explore multiple applications for the method. This kind of research uncovers the unique requirements and challenges each individual structure provides. Also, it is very important to take opportunities, like that fatigue test, which are either in situ or simulations of structures in situ. These real world situations are more dynamic than laboratory tests and explore the complications that arise with taking the method from the lab to the field. One example of this problem is the beat frequency contained in the impedance data discovered during the fatigue test. These real world issues will need to be resolved before the impedance method is ready for deployment on a real structure. 


\section{REFERENCES}

2004. "Wind Power Today." American Wind Energy Association.

2006a. U.S. Department of Energy: Energy Efficiency and Renewable Energy. "Wind and Hydropower Technologies Program: Wind Energy Technology." $<$ http://www1.eere.energy.gov/windandhydro/wind_technologies.html $>$.

2006b. "Wind Power Today: Federal Wind Program Overview: 2006." DOE/GO102006-2319, U.S. Department of Energy: Energy Efficiency and Renewable Energy. 2007a. American Wind Energy Association. "Wind Power Capacity in U.S. Increased 27\% in 2006 and is Expected to Grow an Additional 26\% in 2007."

$<$ http://www.awea.org/newsroom/releases/Wind_Power_Capacity_012307.html $>$ (Jan. $23,2007)$.

2007b. "Wind Power Outlook: 2007." American Wind Energy Association.

2007c. U.S. Department of Energy: Energy Efficiency and Renewable Energy. "Wind Powering America: Installed U.S. Capacity."

$<$ http://www.eere.energy.gov/windandhydro/windpoweringamerica/wind_installed_capac ity.asp $>$.

Beattie, A. G. 1997. "Acoustic Emission Monitoring of A Wind Turbine Blade during a Fatigue Test." 1997 AIAA Aerospace Sciences Meeting, 239-248.

Beattie, A. G., and Rumsey, M. 1999. "Non-Destructive Evaluation of Wind Turbine Blades Using an Infrared Camera." ASME Wind Energy Symposium, 18th, Aerospace Sciences Meeting and Exhibit, 37th, Reno, NV, Jan. 11-14.

Bouteiller, F., Grisso, B. L., Peairs, D. M., and Inman, D. J. 2006. "Broken rail track detection using smart materials." Proceedings of SPIE Smart Structures and Materials/NDE, San Diego, CA, Feb. 26 - Mar. 2. 
Ghoshal, A., Sundaresan, M. J., Schulz, M. J., and Pai, P. F. 2000. "Structural Health Monitoring Techniques for Wind Turbine Blades." Journal of Wind Engineering and Industrial Aerodynamics, 85, 309-324.

Gieske, J. H., and Rumsey, M. A. 1997. "Non-destructive Evaluation (NDE) Of Composite/Metal Bond Interface Of A Wind Turbine Blade Using An Acousto-ultrasonic Technique." 1997 AIAA Aerospace Sciences Meeting, 249-254.

Grisso, B. L., and Inman, D. J. 2006. "Impedance-Based Structural Health Monitoring of Thermal Protection Systems." Proceedings of SPIE Smart Structures and Materials/NDE, San Diego, CA, Feb. 26 - March 2.

Grisso, B. L., Inman, D.J. 2005a. "Developing an Autonomous On-Orbit ImpedanceBased SHM System for Thermal Protection Systems." Structural Health Monitoring 2005: Advancements and Challenges for Implementation, Proceedings of the 5th International Workshop on Structural Health Monitoring, Stanford University, Stanford, CA, 435.

Grisso, B. L., Martin, L.A., Inman, D.J. 2005b. "A Wireless Active Sensing System for Impedance-Based Structural Health Monitoring." Proceedings of IMAC XXIII, Orlando, FL, January 31-February 3, 2005.

Grisso, B. L., Peairs, D.M., Inman, D.J. 2004a. "Detecting Damage in Graphite/Epoxy Composites Using Impedance-based Structural Health Monitoring." Applied Mechanics and Materials, 1-2, 185-190.

Grisso, B. L., Peairs, D.M., Inman, D.J. 2004b. "Impedance-based Health Monitoring of Composites." Proceedings of IMAC XXII, Dearborn, MI, Jan. 26-29, 2004.

Gross, E., Simmermacher, T., Rumsey, M. A., and Zadok, R. I. 1998. "Application of Damage Detection Techniques Using Wind Turbine Modal Data." SAND98-2823c, Sandia National Laboratories.

Hau, E. 2006. Wind Turbines: Fundamentals, Technologies, Application, Economics, 2nd Ed., Springer-Verlag, Berlin. 
Inman, D. J., and Grisso, B. L. 2006. "Towards autonomous sensing." Proceedings of SPIE: Smart Structures and Materials/NDE, San Diego, CA, Feb. 26 - Mar. 2.

Kim, J., Grisso, B. L., Ha, D. S., and Inman, D. J. 2007. "A System-On-Board Approach for Impedance-Based Structural Health Monitoring." SPIE Smart Structures and Materials and Nondestructive Evaluation and Health Monitoring, San Diego, CA, March $18-22$.

Kirikera, G. R., Schulz, M. J., and Sundaresan, M. J. 2007. "Multiple Damage Identification on a Wind Turbine Blade Using a Structural Neural System." SPIE Smart Structures and Materials \& Nondestructive Evaluation and Health Monitoring, San Diego, CA, March 18 - 22.

Koo, K.-Y., Park, S., Lee, J.-J., et al. 2007. "Impedance-based Structural Health Monitoring Considering Temperature Effects." SPIE Smart Structures and Materials and Nondestructive Evaluation and Health Monitoring, San Diego, CA, March 18-22.

Liang, C., Sun, F.P., and Rogers, C.A. 1994. "Coupled Electromechanical Analysis of Adaptive Material Systems - Determination of the Actuator Power Consumption and System Energy Transfer." Journal of Intelligent Material Systems and Structures, 5, 1220.

Locke, J., Valencia, U., and Ishikawa, K. 2003. "Design Studies for Twist-Coupled Wind Turbine Blades." 41st Aerospace Sciences Meeting and Exhibit, Reno, NV, Jan. 6-9.

Mascarenas, D., Todd, M., Park, G., and Farrar, C. 2006. "A Miniaturized

Electromechanical Impedanced-Based Node for the Wireless Interrogation of Structural Health." Proceedings of SPIE, 6177.

Overlay, T. G., Park, G., Farrar, C. R., and Allemang, R. J. 2007. "Compact Hardware Development of SHM and Sensor Diagnostics using Admittance Measurements." Internation Model Analysis Conference, Orlando, FL, February 19-22. 
Paquette, J., Dam, J. V., and Hughes, S. 2007a. "Structural Testing of 9 m Carbon Fiber Wind Turbine Research Blades." 45th AIAA Aerospace Sciences Meeting and Exhibit, Reno, Nevada, Jan. 8-11.

Paquette, J., Laird, D., Griffith, D. T., and Rip, L. 2006. "Modeling and Testing of 9m Research Blades." 44th AIAA Aerospace Sciences Meeting and Exhibit, Reno, NV, Jan. 9-12.

Paquette, J., Rumsey, M. A., Dam, J. V., and Hughes, S. 2007b. "Blade Fatigue Test Test Plan for the TPI/Sandia TX-100 Blade." Internal Document, National Renewable Energy Laboratory and Sandia National Laboratories.

Park, G., Farrar, C. R., Scalea, F. L. D., and Coccia, S. 2006a. "Performance assessment and validation of piezoelectric active-sensors in structural health monitoring." Smart Materials and Structures, 15, 1673-1683.

Park, G., Kabeya, K., Cudney, H. H., and Inman, D. J. 1999. "Impedance-Based Structural Health Monitoring for Temperature Varying Applications." JSME International Journal, Series A, 42(2), 249-258.

Park, G., Rutherford, A. C., Wait, J. R., et al. 2005. "High-Frequency Response Functions for Composite Plate Monitoring with Ultrasonic Validation." AIAA Journal, 43(11), 2431-2437.

Park, G., Sohn, H. Farrar, C.R. And Inman, D.J. 2003. "Overview of Piezoelectric Impedance-based Health Monitoring and Path Forward." The Shock and Vibration Digest, 35(6), 451-463.

Park, S., Grisso, B. L., Inman, D. J., and Yun, C.-B. 2007a. "MFC-Based Structural Health Monitoring Using a Miniaturized Impedance Measuring Chip for Corrosion Detection." Research in Nondestructive Evaluation, 18, 139-150.

Park, S., Lee, J.-J., Yun, C.-B., and Inman, D. J. 2007b. "Electro-Mechanical ImpedanceBased Wireless Structural Health Monitoring Using PCA-Data Compression and k- 
means Clustering Algorithms." Journal of Intelligent Material Systems and Structures, $5(0)$.

Park, S., Yun, C.-B., and Inman, D. J. 2006b. "A Self-contained Active Sensor System for Health Monitoring of Civil Infrastructures." IEEE Sensors, Daegu, Korea, October $22-25$.

Peairs, D. 2006. "High Frequency Modeling and Experimental Analysis for Implementation of Impedance-based Structural Health Monitoring," PhD Dissertation, Virginia Polytechnic Institute and State University, Blacksburg, Virginia.

Peairs, D., Park, G., and Inman, D.J. 2004. "Improving Accessibility of the ImpedanceBased Structural Health Monitoring Method." Journal of Intelligent Material Systems and Structures, 15, 129-139.

Peairs, D. M., Grisso, B. L., Margasahayam, R. N., et al. 2004. "Impedance-based Health Monitoring of Space Shuttle Ground Structures." Proceedings of SPIE Smart Structures and Materials, San Diego, CA, March 14-18.

Peairs, D. M., Tarazaga, P. A., and Inman, D. J. 2006. "A Study of the Correlation between PZT and MFC Resonance Peaks and Damage Detection Frequency Intervals Using the Impedance Method." International Conference on Noise and Vibration Engineering, Leuven, Belgium, September 18-20, 909-924.

Pitchford, C. W., Grisso, B. L., and Inman, D. J. 2007. "Impedance-based structural health monitoring of wind turbine blades." SPIE Smart Structures and Materials and Nondestructive Evaluation and Health Monitoring, San Diego, CA, March 18-22.

Reetz, J. 2007. "Structural Health Monitoring of Offshore Wind Turbines using the Multiparameter Eigenvalue Problem." Abstract submitted for International Operational Modal Analysis Conference, Copenhagen, Denmark, April 30 - May 1.

Rolfes, R., Gerasch, W., Haake, G., et al. 2006. "Early Damage Detection System for Towers and Rotor Blades of Offshore Wind Turbines." Third European Workshop on Structural Health Monitoring, Granada, Spain, July 5-7, 455-462. 
Rumsey, M. A., Hurtado, J., Hansche, B., et al. 1997. "In-Field Use of Laser Dopler Vibrometer on Wind Turbine Blade." SAND97-2745c, Sandia National Laboratories.

Schulz, M. J., and Sundaresan, M. J. 2006. "Smart Sensor System for Structural Condition Monitoring of Wind Turbines: May 30, 2000 - April 30, 2006." NREL/SR-50040089, National Renewable Energy Laboratory.

Simmermacher, T., James, G. H., and Hurtado, J. E. 1997. "Structural Health Monitoring of Wind Turbines." Proceedings of the International Workshop on Structural Health Monitoring, Stanford, CA, September 18-20, 1997, 788-797.

Simmers, G. E. 2005. "Impedance-Based Structural Health Monitoring to Detect Corrosion," Master's Thesis, Virginia Polytechnic Institute and State University, Blacksburg, Virginia.

Sodano, H. A., Park, G., and Inman, D.J. 2003. "An Investigation into the Performance of Macro-Fiber Composites for Sensing and Structural Vibration Applications." Mechanical Systems and Signal Processing, 18, 683-697.

Sundaresan, M. J., Schulz, M. J., and Ghoshal, A. 2002. "Structural Health Monitoring Static Test of a Wind Turbine Blade: August 1999." NREL/SR-500-28719, National Renewable Energy Laboratory.

Sundaresan, M. J., Schulz, M. J., Ghoshal, A., et al. 2001. "An Intelligent Blade for Wind Turbines." 39th AIAA/ASME Aerospace Sciences Meeting and Exhibit, Wind Energy Symposium, Reno, Nevada, 8-11 January 2001.

Sundaresan, M. J., Schulz, M. J., Hill, J., et al. 1999. "Damage Detection on a Wind Turbine Blade Section." IMAC XVII, Kissimmee, FL, Feb. 8-11, 1359-1365.

Sutherland, H. 1999. "On the Fatigue Analysis of Wind Turbines." SAND99-0089, Sandia National Laboratories. 
Sutherland, H., Beattie, A., Hansche, B., et al. 1993. "The Application of Non-

Destructive Techniques to the Testing of a Wind Turbine Blade." SAND93-1380, Sandia National Laboratories.

Tarazaga, P. A., Peairs, D. M., Wilkie, W. K., and Inman, D. J. 2006. "Structural Health Monitoring of an Inflatable Boom Subjected to Simulated Micrometeoroid/Orbital Debris Damage." SPIE 11th International Symposium on Nondestructive Evaluation for Health Monitoring and Diagnostics, San Diego, CA, February 26-March 2

White, J. R. 2006. "Impact and Thermal Damage Identification in Metallic Honeycomb Thermal Protection System Panels using Active Distributed Sensing with the Method of Virtual Forces," Master's Thesis, Purdue University, West Lafayette, Indiana.

White, J. R., Adams, D. E., and Jata, K. 2006. "Damage Identification in a Sandwich Plate Using the Method of Virtual Forces." Proceeding of the International Modal Analysis Conference, St. Louis, MO.

Zayas, J. R., Paquette, J., and Werlink, R. J. 2007. "Evaluation of NASA PZT

Sensor/Actuator for Structural Health Monitoring of a Wind Turbine Blade." 45th AIAA Aerospace Sciences Meeting and Exhibit, Reno, NV, January 8-11. 


\section{APPENDIX A: IBSHM, indirect damage, impedance plots}
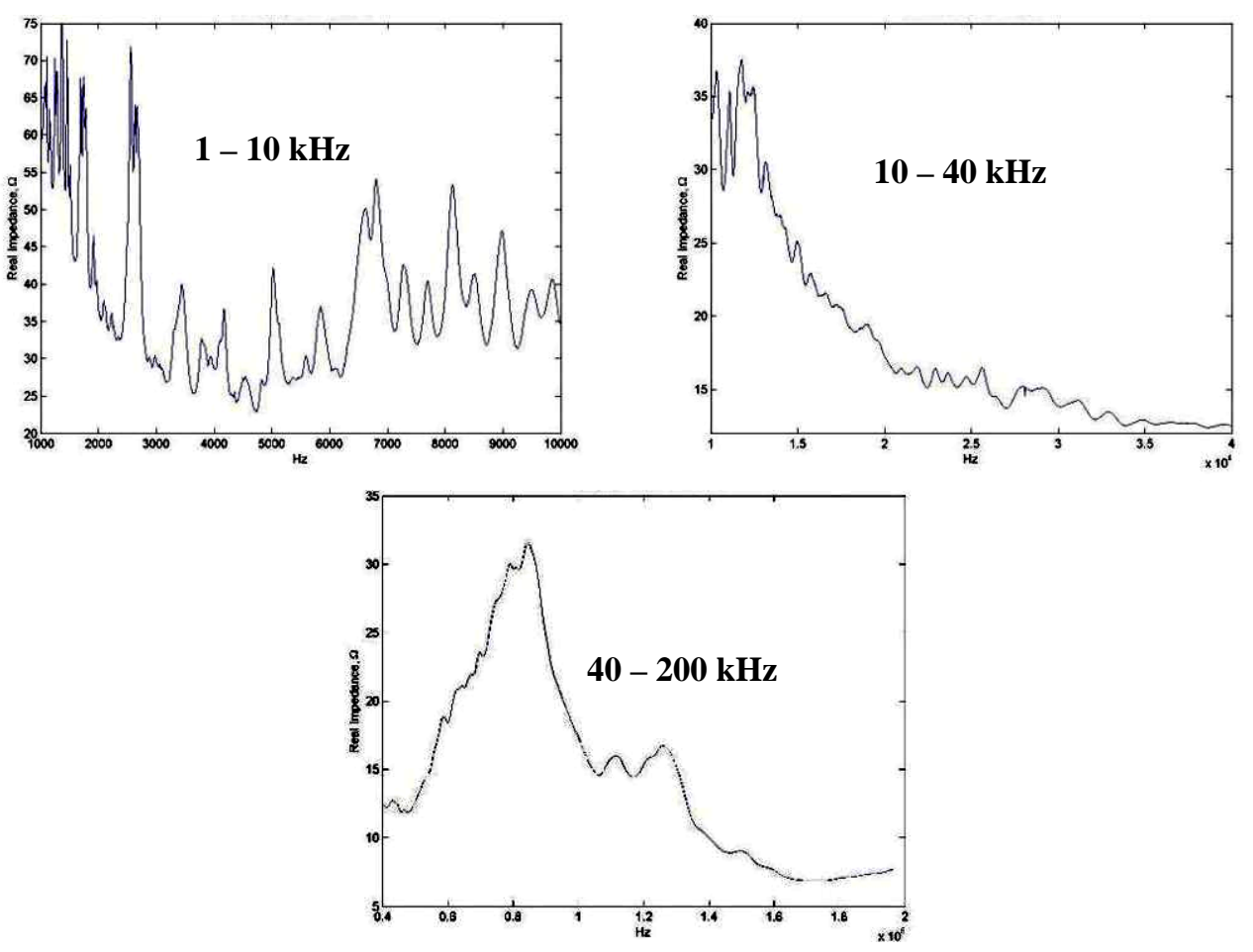

Top/Skin Baseline Impedance
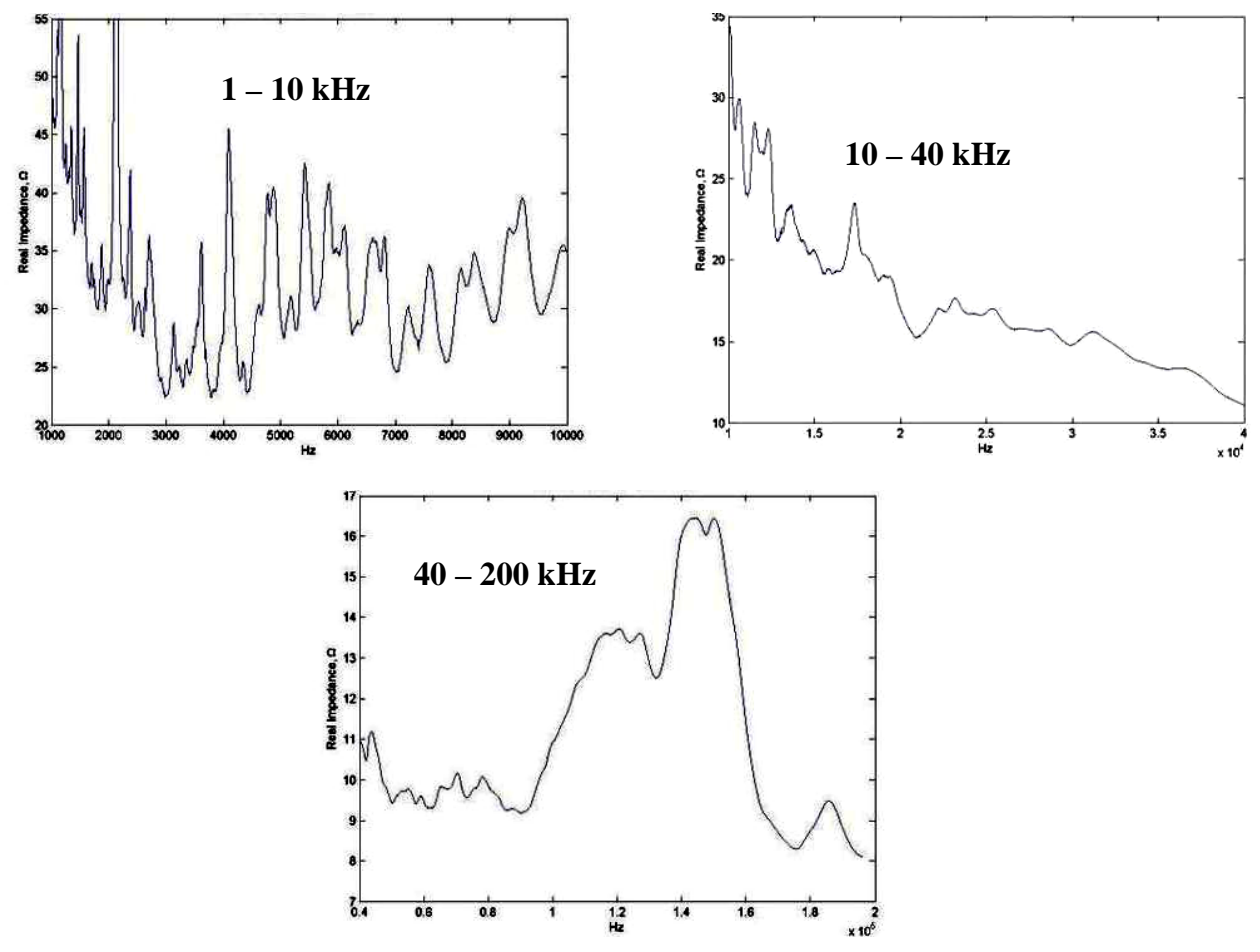

Carbon Baseline Impedance 
Note: Throughout impedance signature appendices, baseline is average of multiple impedance signatures and mass 1 , mass 2 , and mass 3 refer to locations 1-3.

\section{Added mass, Skin}
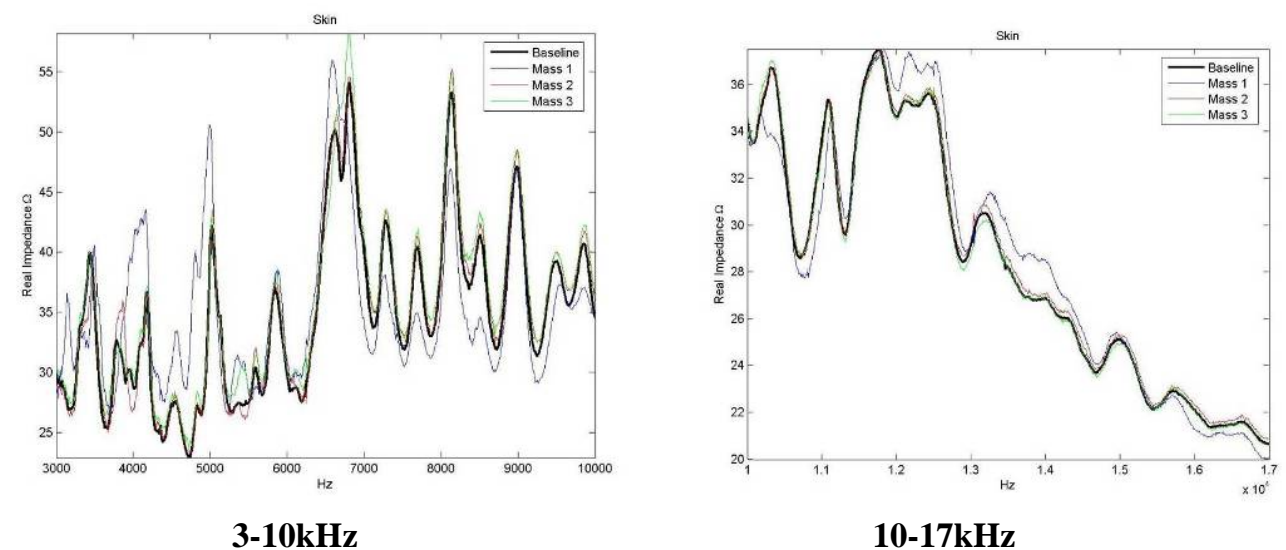

$10-17 \mathrm{kHz}$

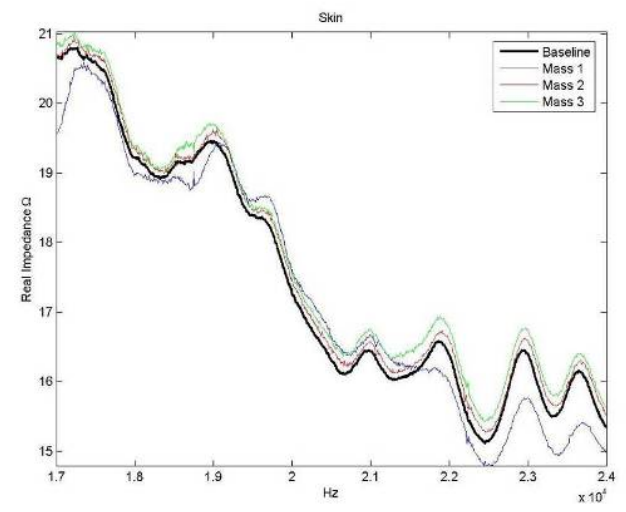

$17-24 k H z$

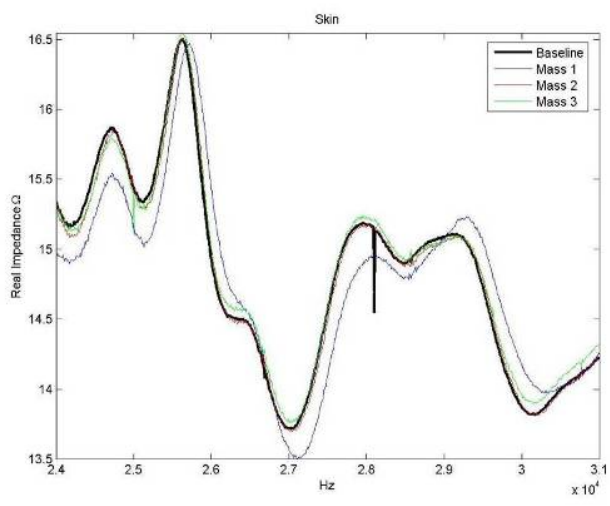

$24-31 \mathrm{kHz}$

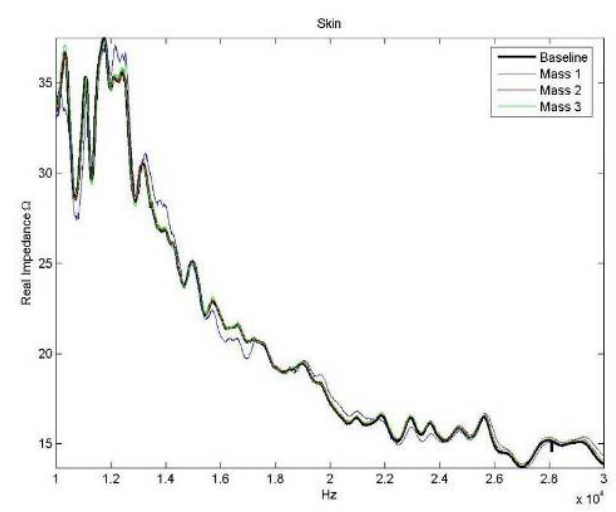

$10-30 \mathrm{kHz}$

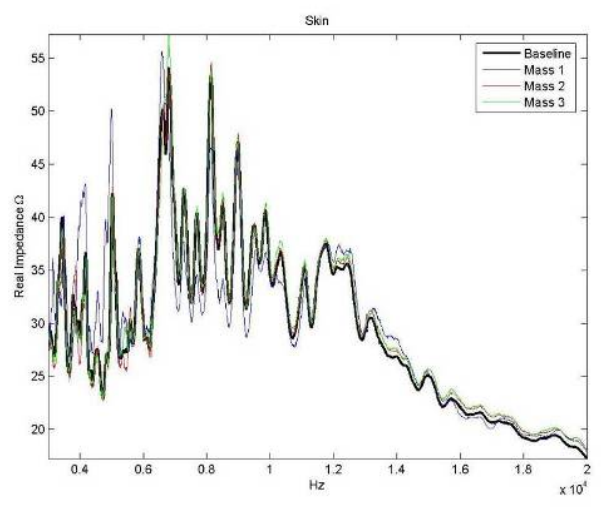

3-20kHz 


\section{Added mass, Spar}
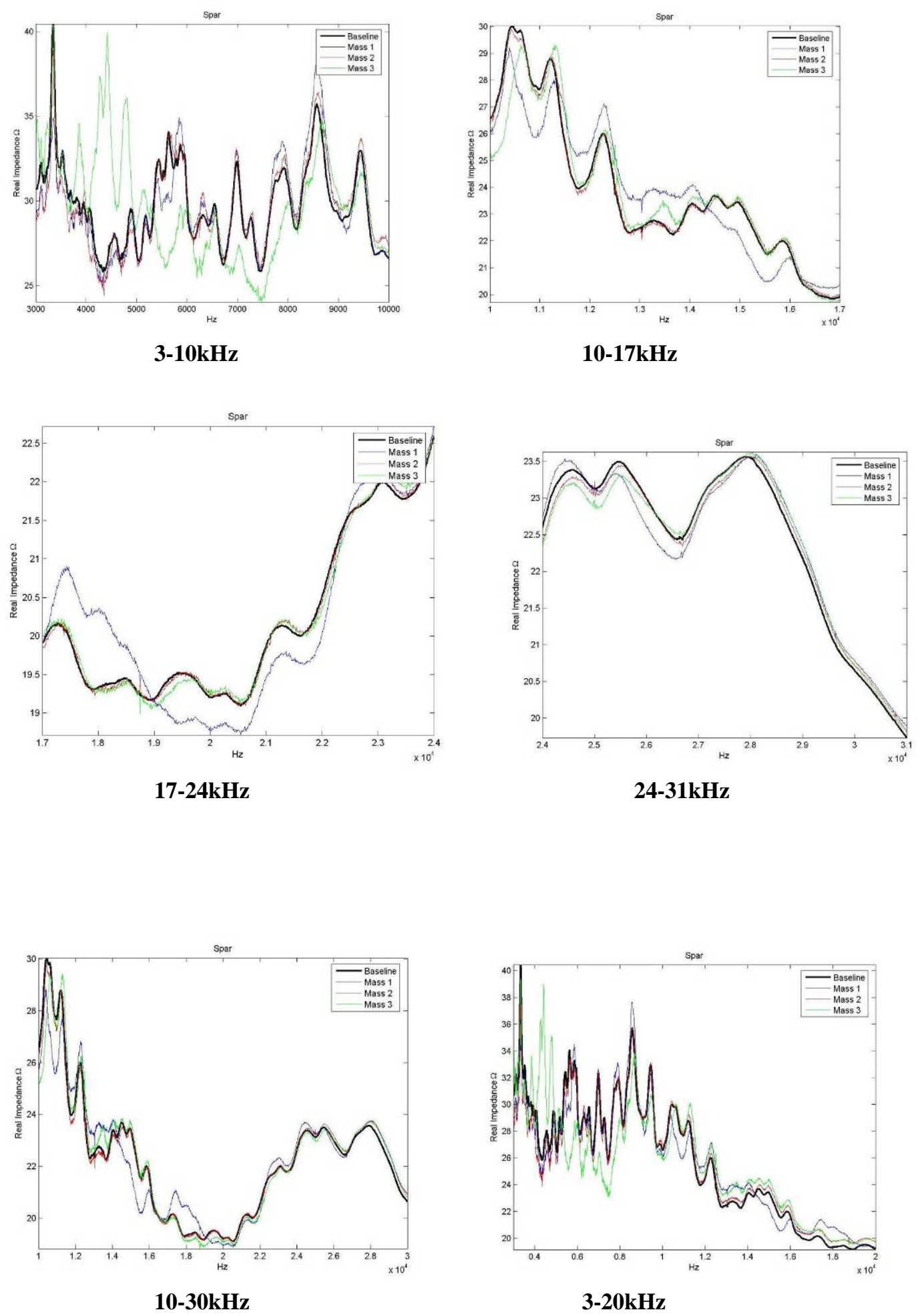
Added mass, Carbon
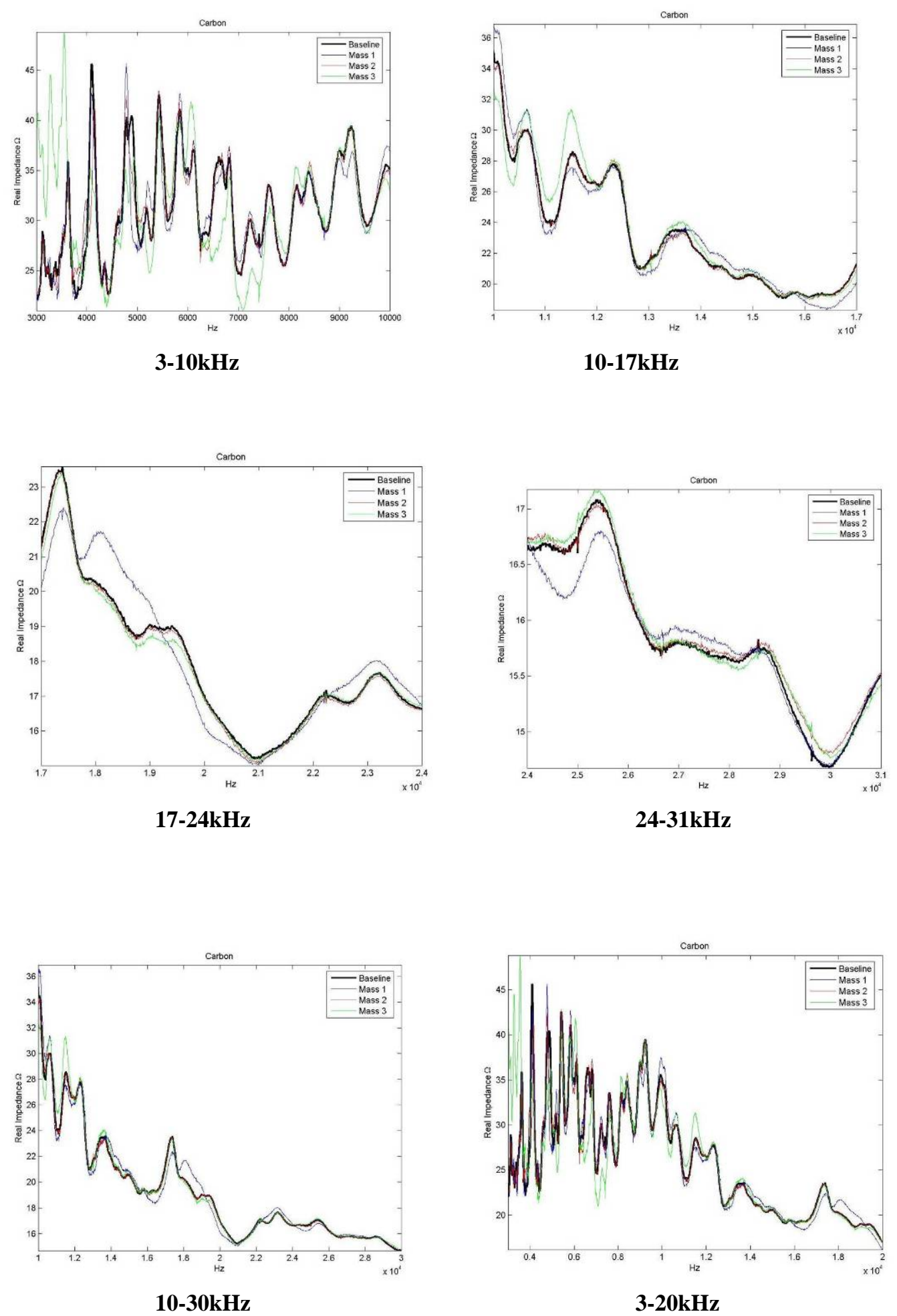
Added stiffness, Skin
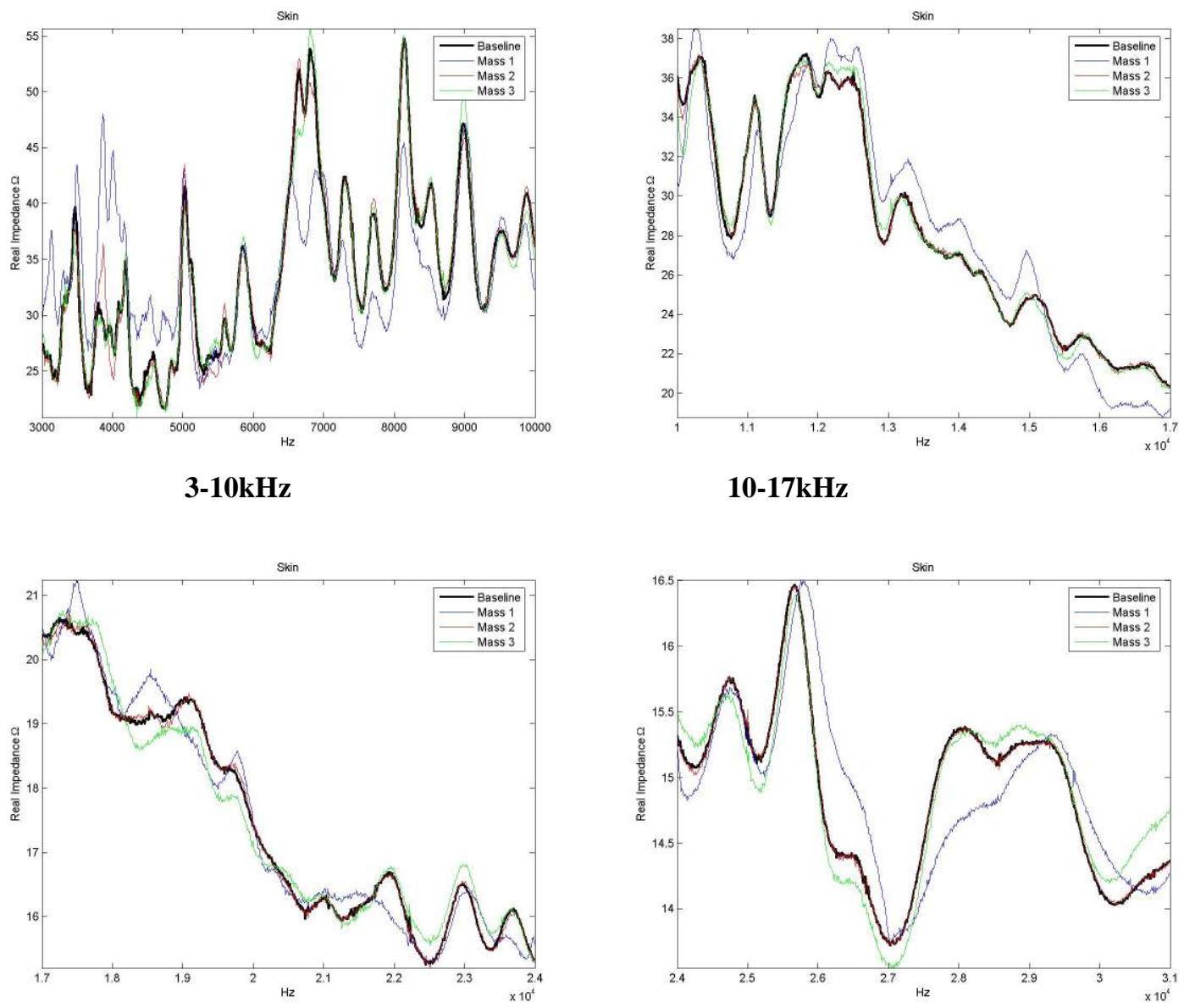

$17-24 k H z$

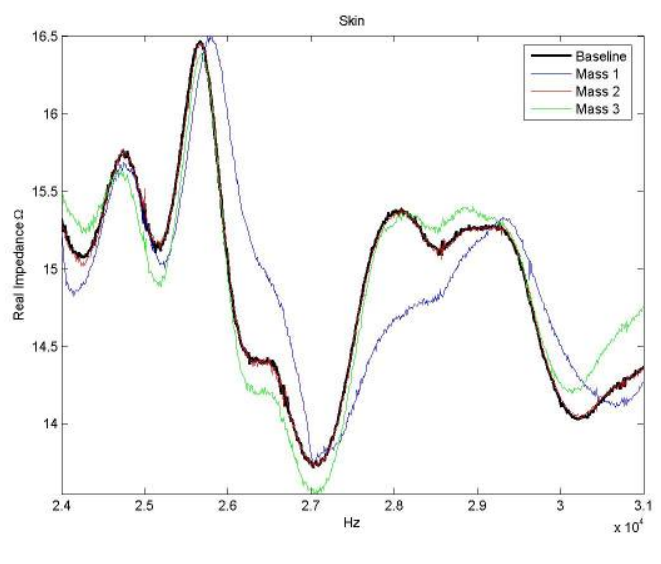

24-31kHz

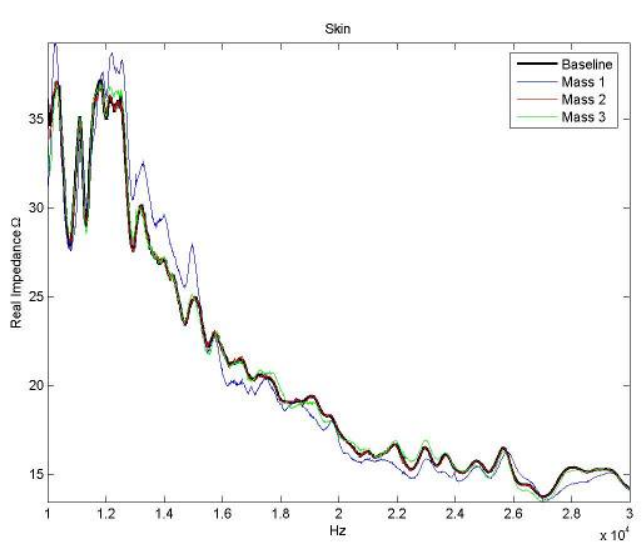

$10-30 \mathrm{kHz}$

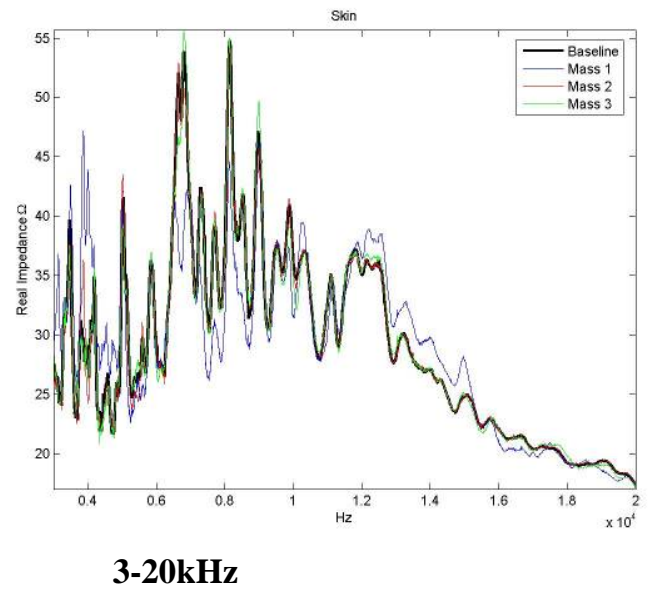


Added stiffness, Spar
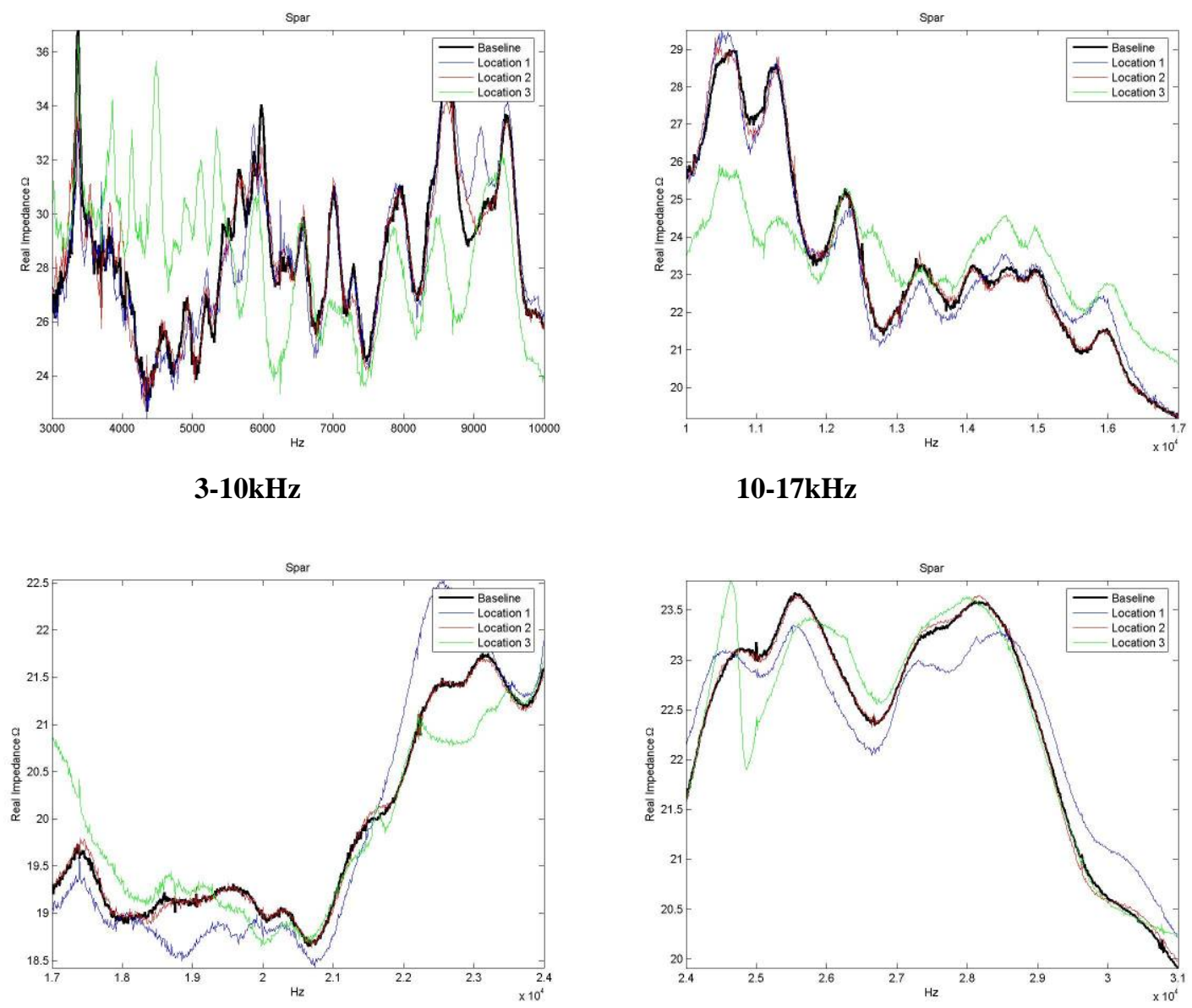

$17-24 k H z$

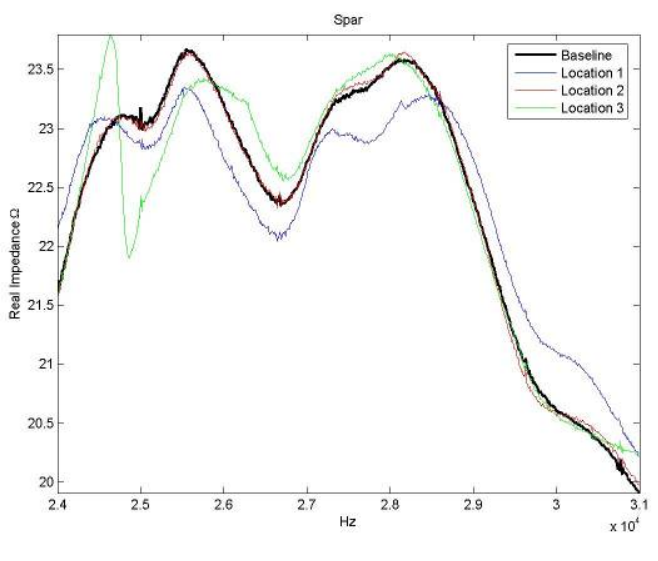

$24-31 \mathrm{kHz}$
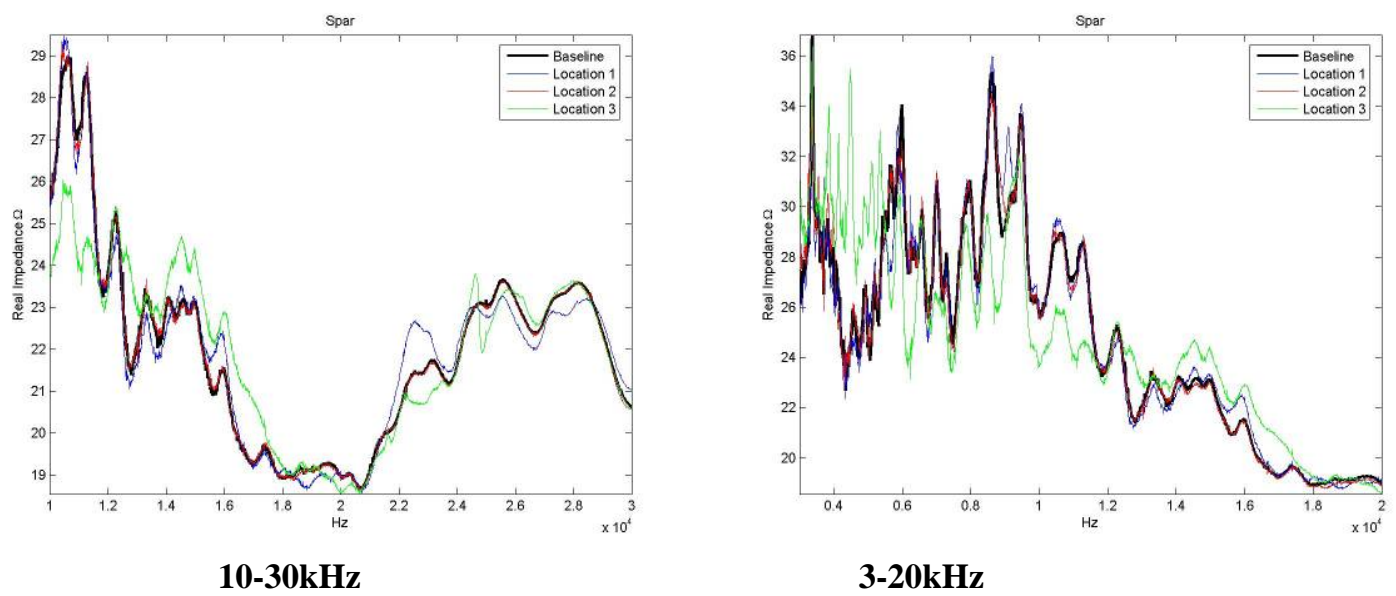
Added stiffness, Carbon
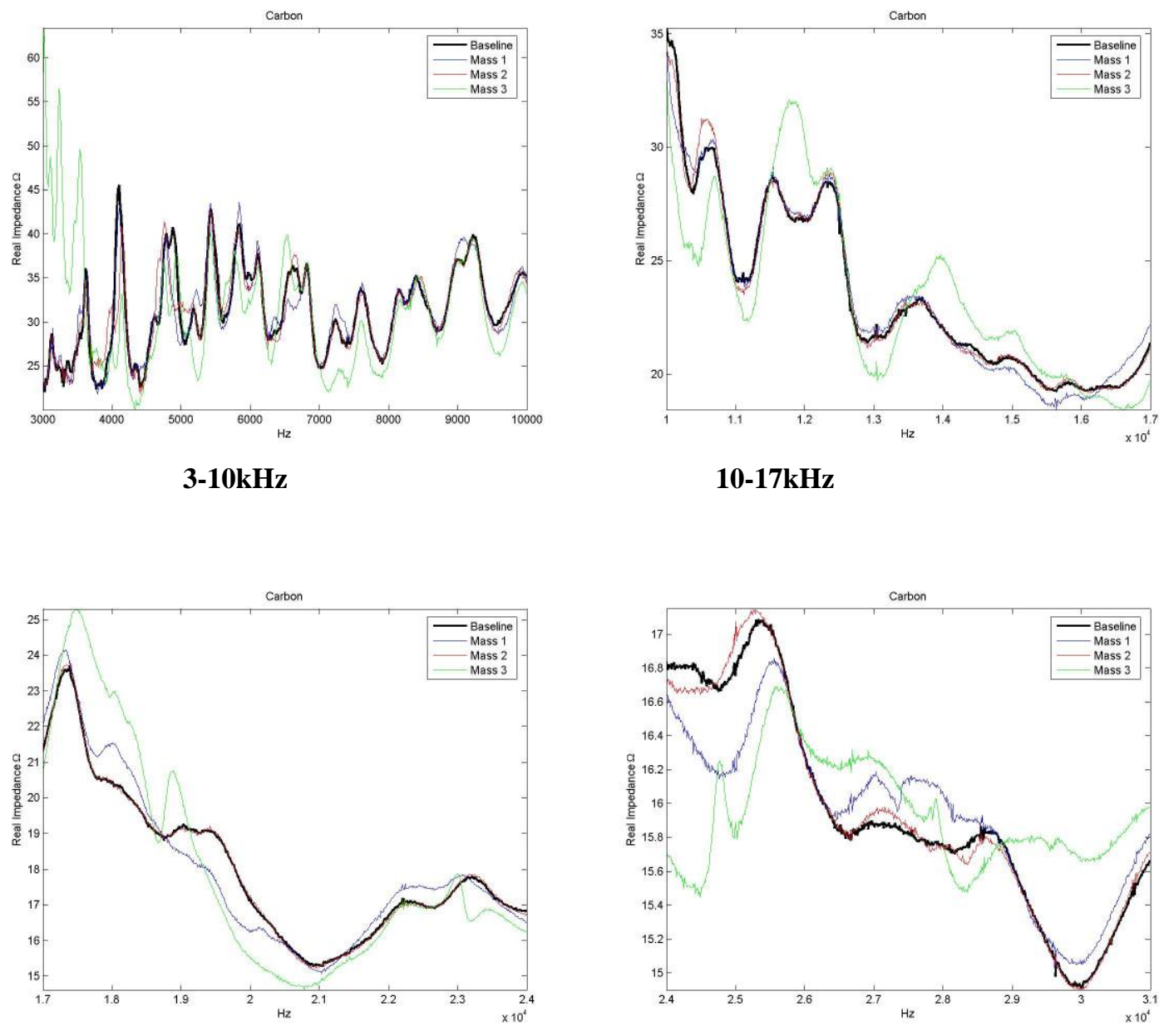

$17-24 \mathrm{kHz}$
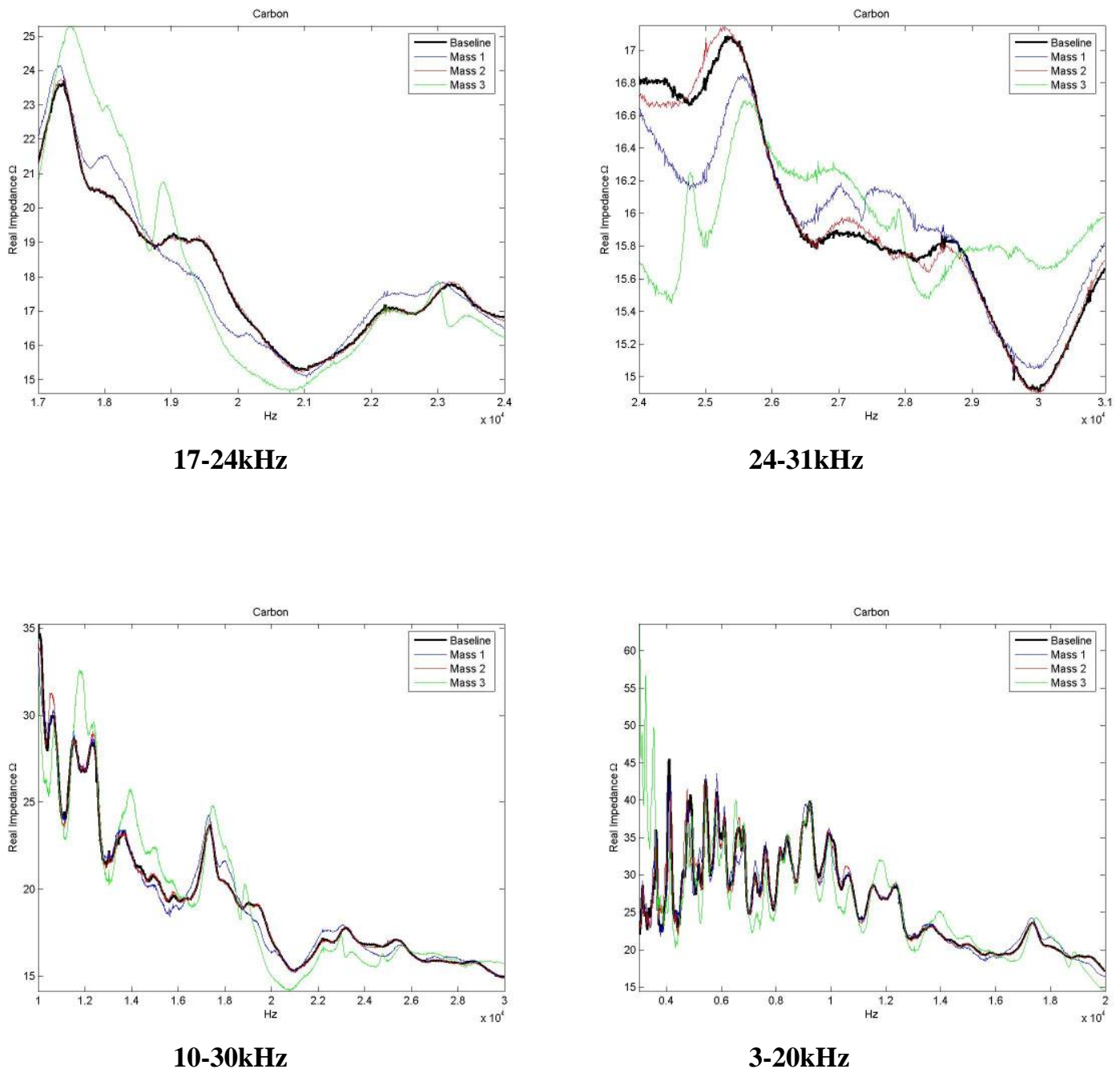
Added stiffness, Higher Frequency, Skin

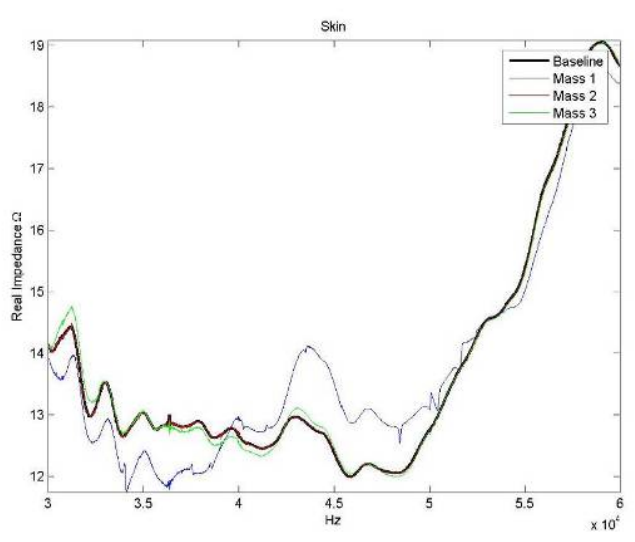

$30-60 \mathrm{kHz}$

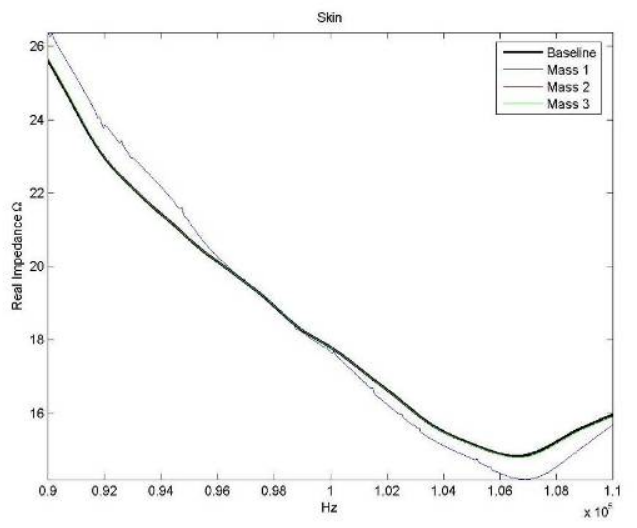

90-110kHz

Added stiffness, Higher Frequency, Spar

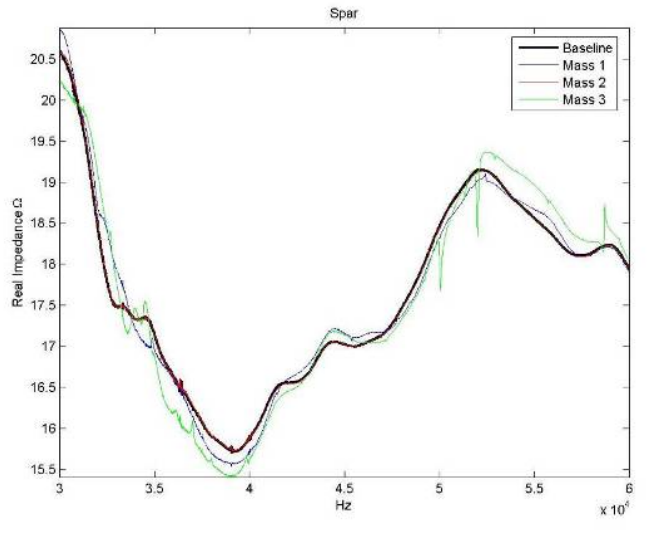

$30-60 \mathrm{kHz}$

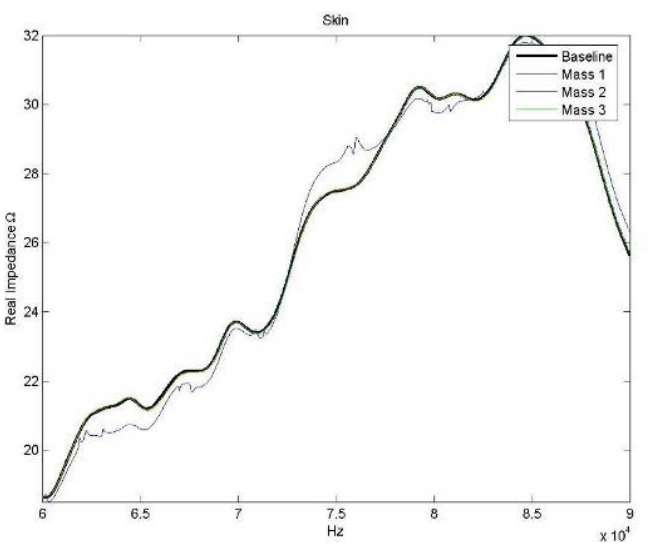

$60-90 \mathrm{kHz}$

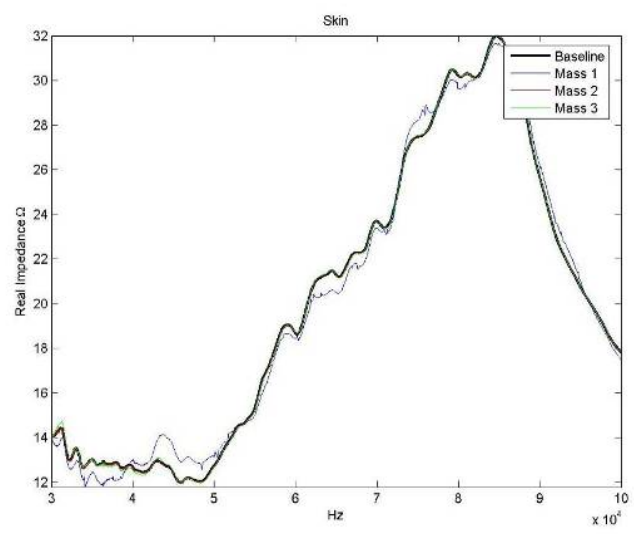

$30-100 \mathrm{kHz}$

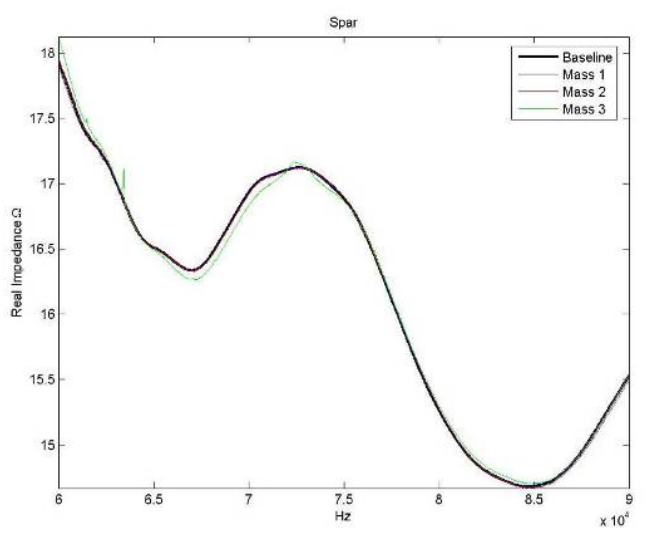

$60-90 \mathrm{kHz}$ 


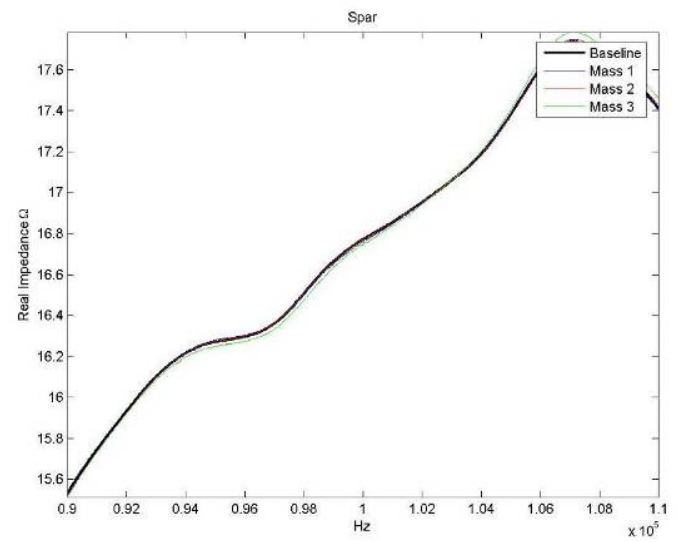

90-110kHz

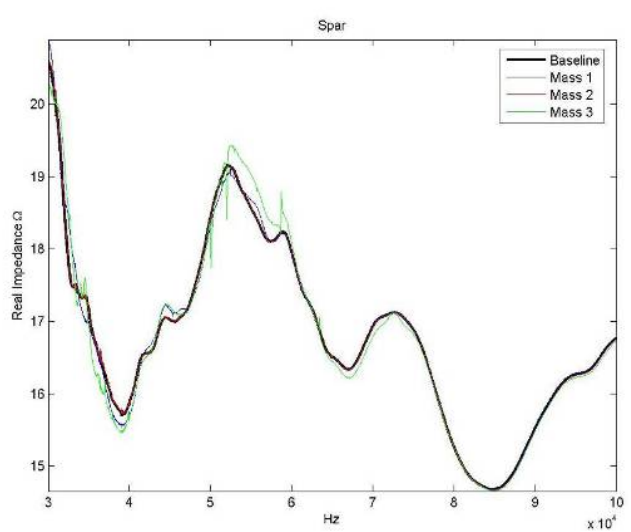

$30-100 \mathrm{kHz}$

\section{Added stiffness, Higher Frequency, Carbon}

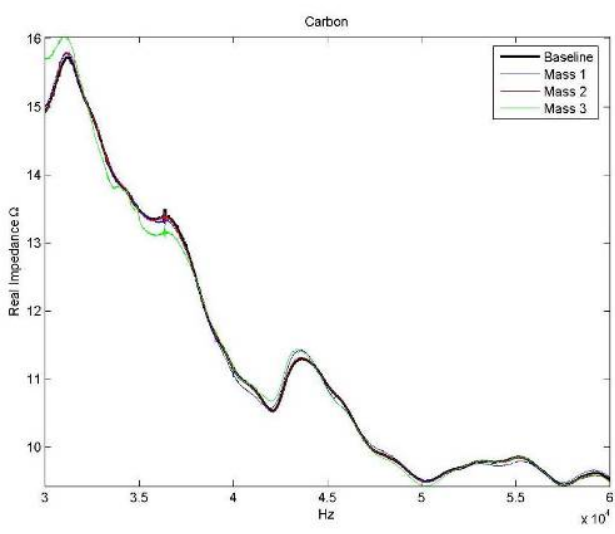

$30-60 \mathrm{kHz}$

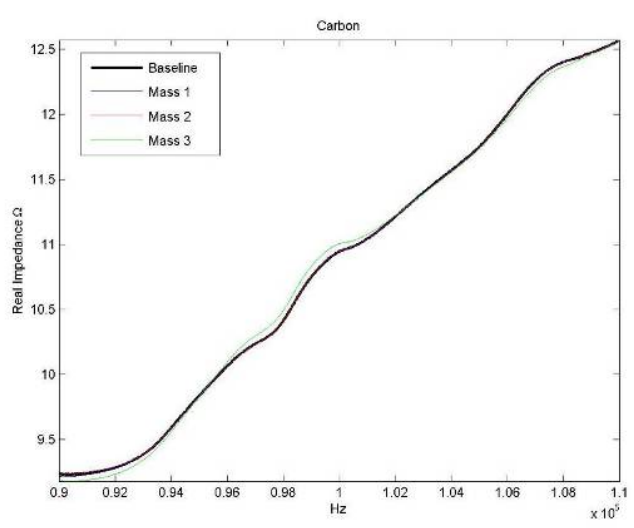

$90-110 \mathrm{kHz}$

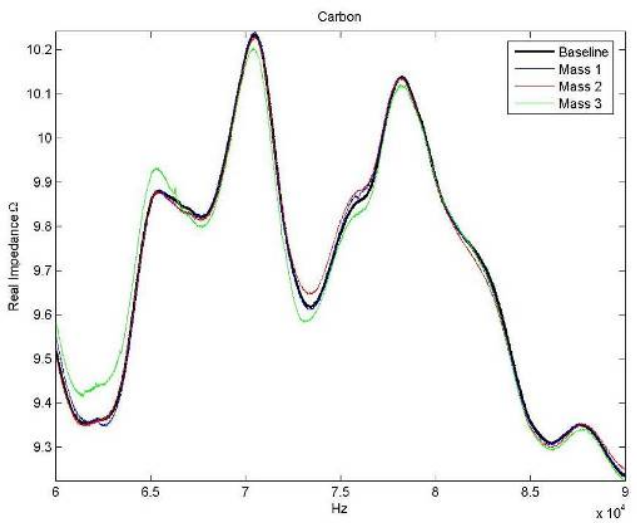

$60-90 \mathrm{kHz}$

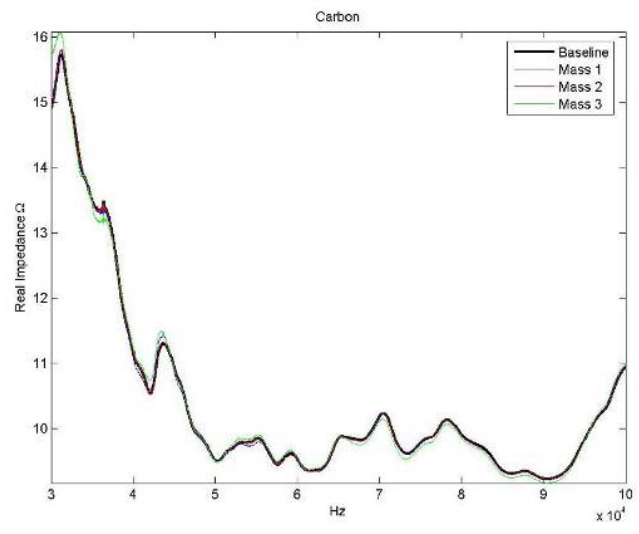

$30-100 \mathrm{kHz}$ 


\section{APPENDIX B: IBSHM, actual damage, impedance plots}

\section{Location 1 down blade}

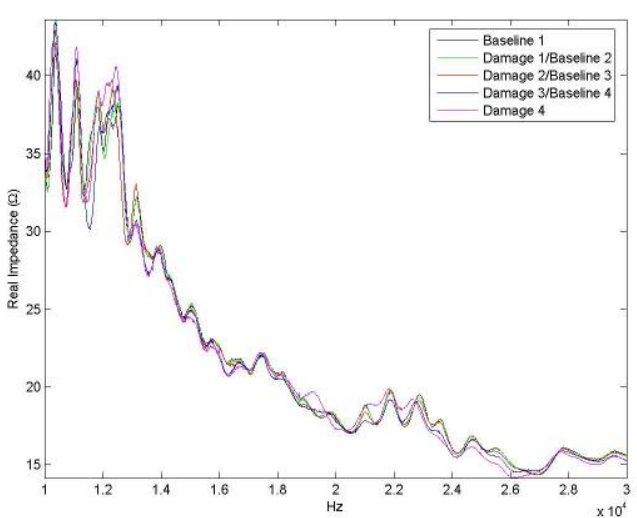

Skin, 10-30kHz
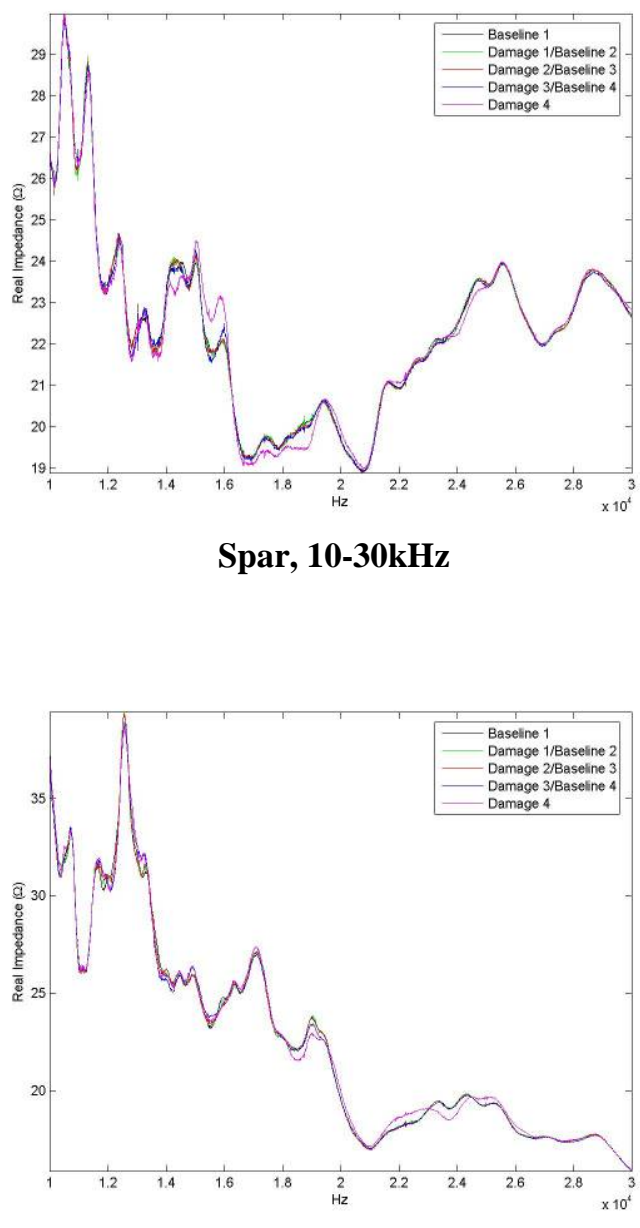

Carbon, 10-30kHz

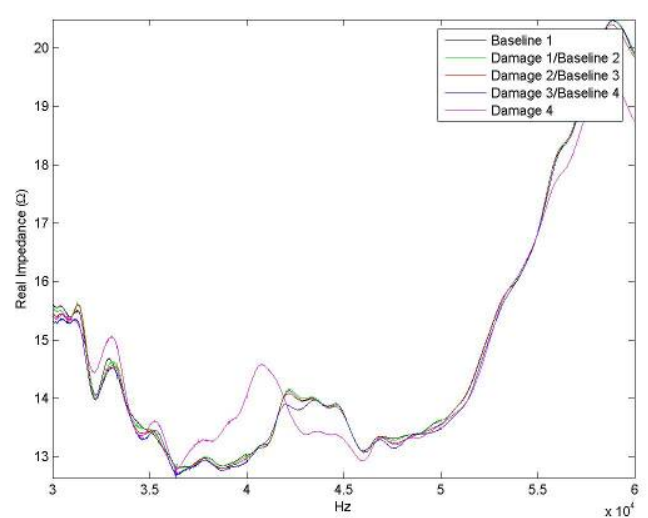

Skin, $30-60 \mathrm{kHz}$

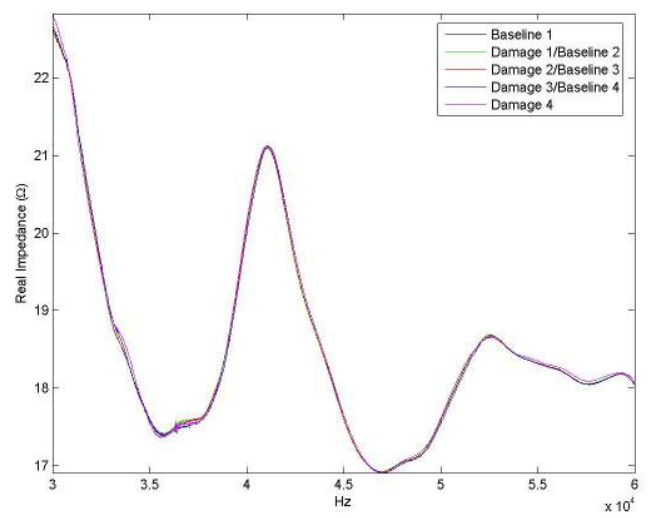

Spar, $30-60 \mathrm{kHz}$

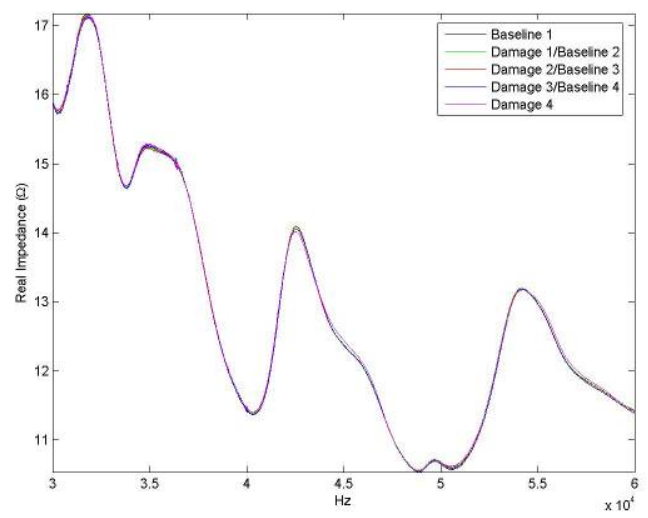

Carbon, 30-60kHz 


\section{Location 1 split}

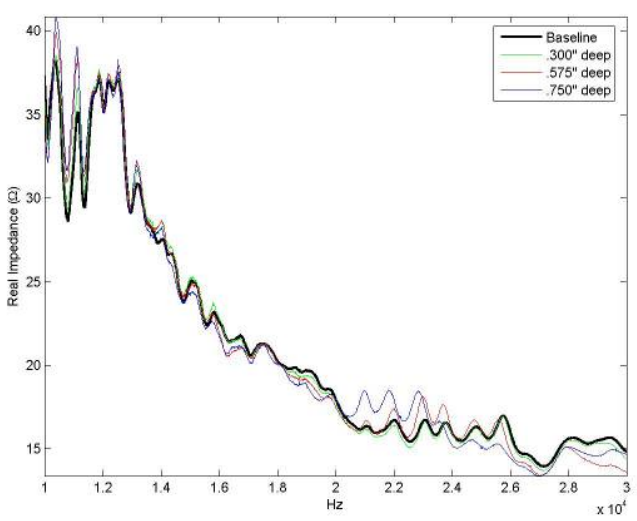

Skin, 10-30kHz
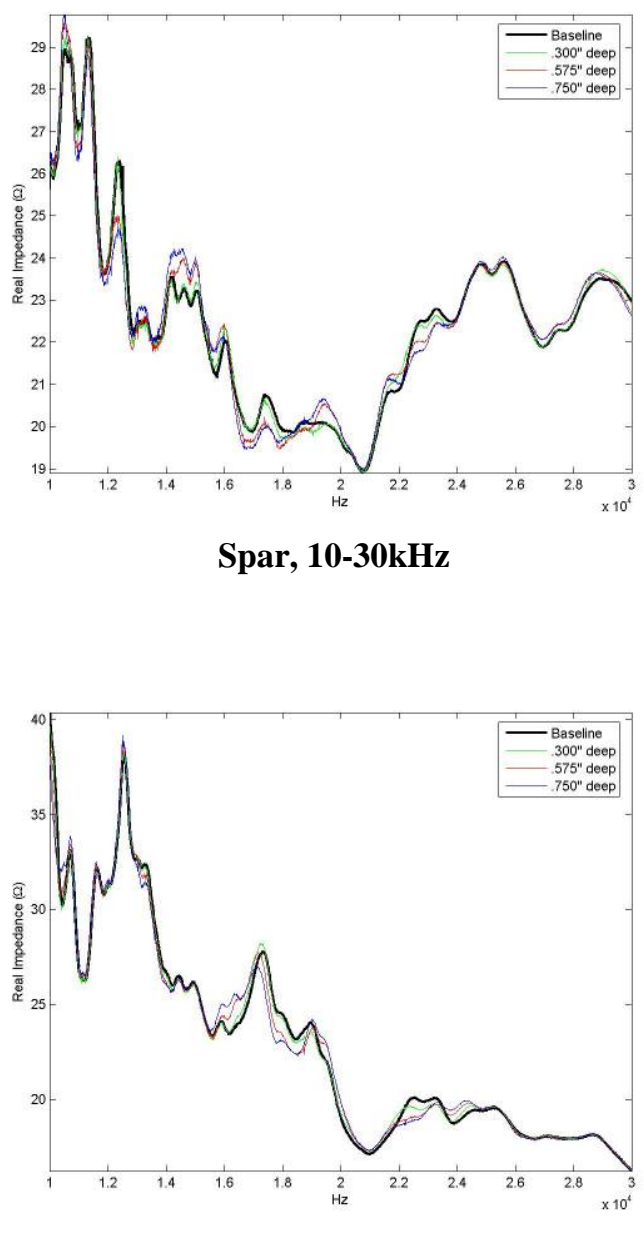

Carbon, $10-30 \mathrm{kHz}$

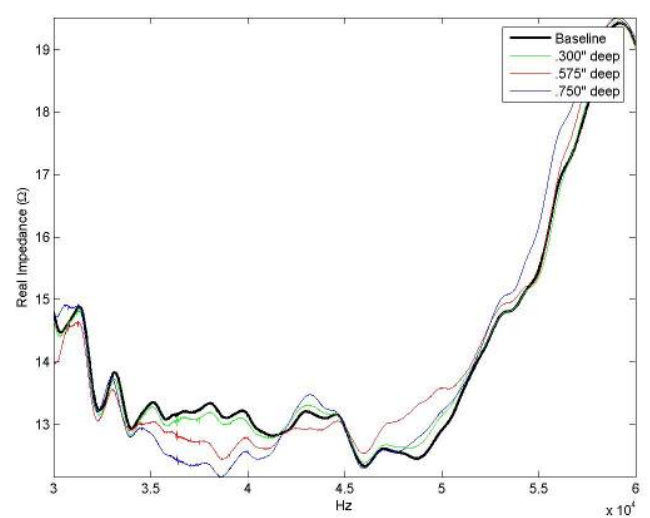

Skin, $30-60 \mathrm{kHz}$

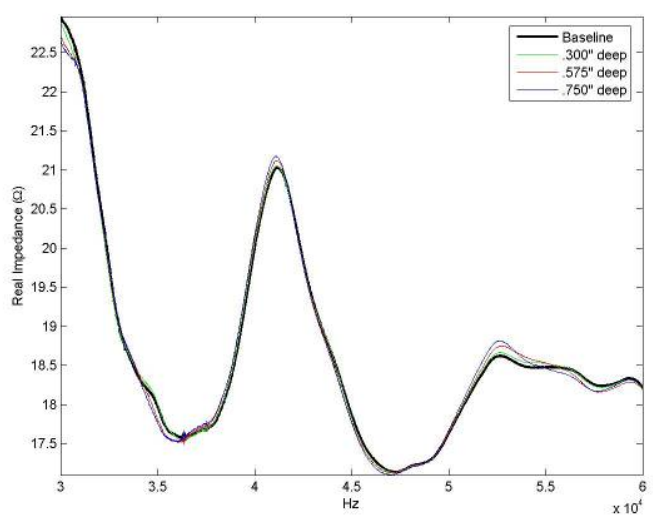

Spar, $30-60 \mathrm{kHz}$

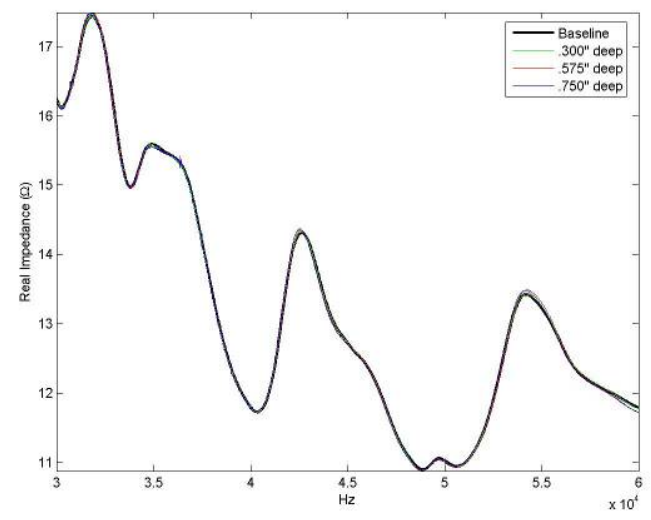

Carbon, 30-60kHz 


\section{Location 3 down blade}

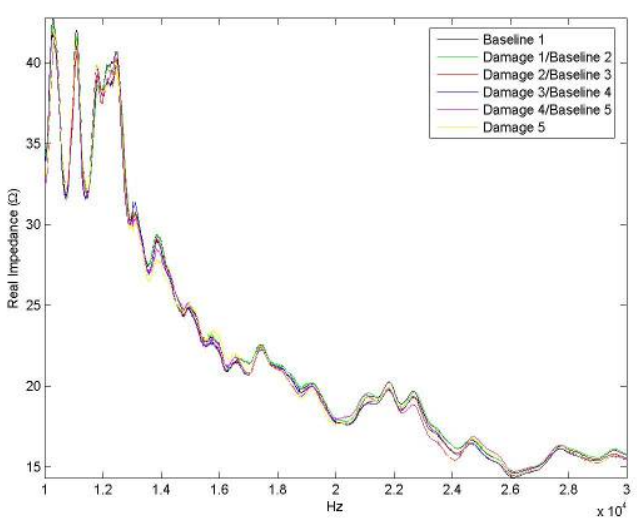

Skin, 10-30kHz
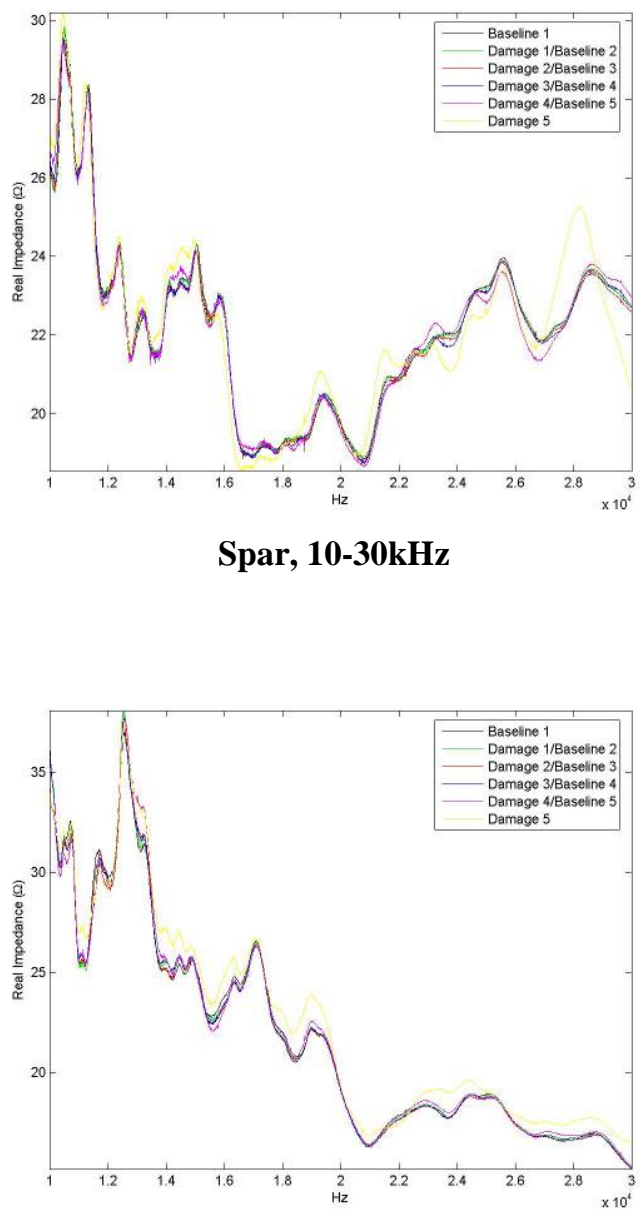

Carbon, 10-30kHz

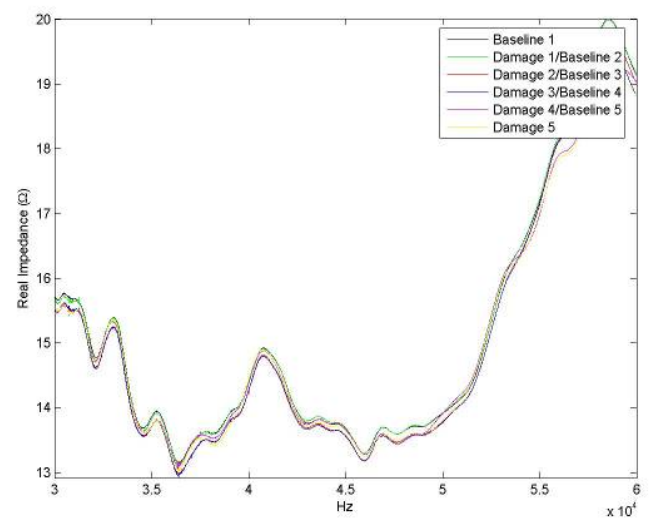

Skin, $30-60 \mathrm{kHz}$

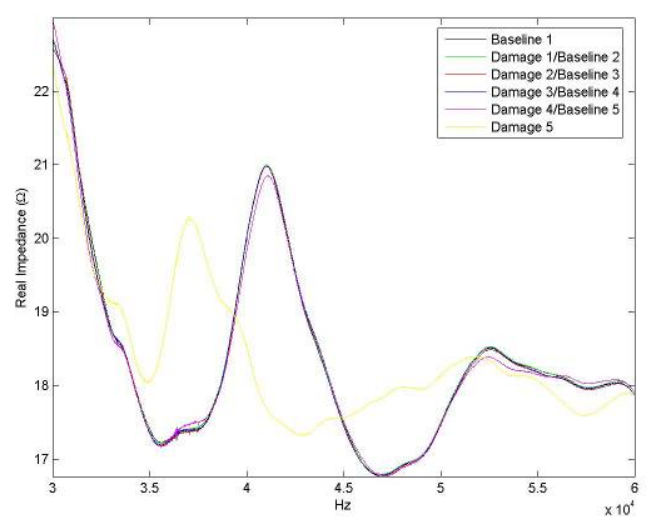

Spar, $30-60 \mathrm{kHz}$

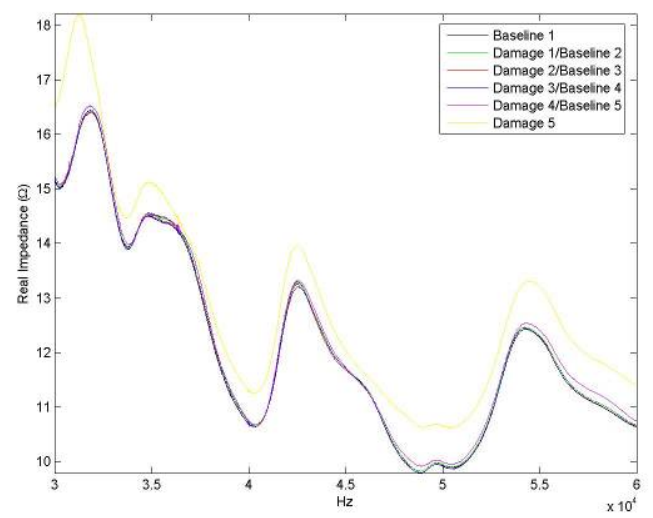

Carbon, 30-60kHz 


\section{Location 3 holes}

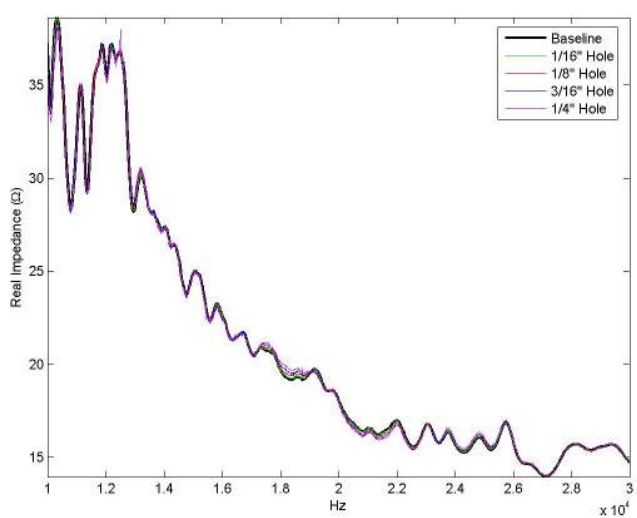

Skin, 10-30kHz
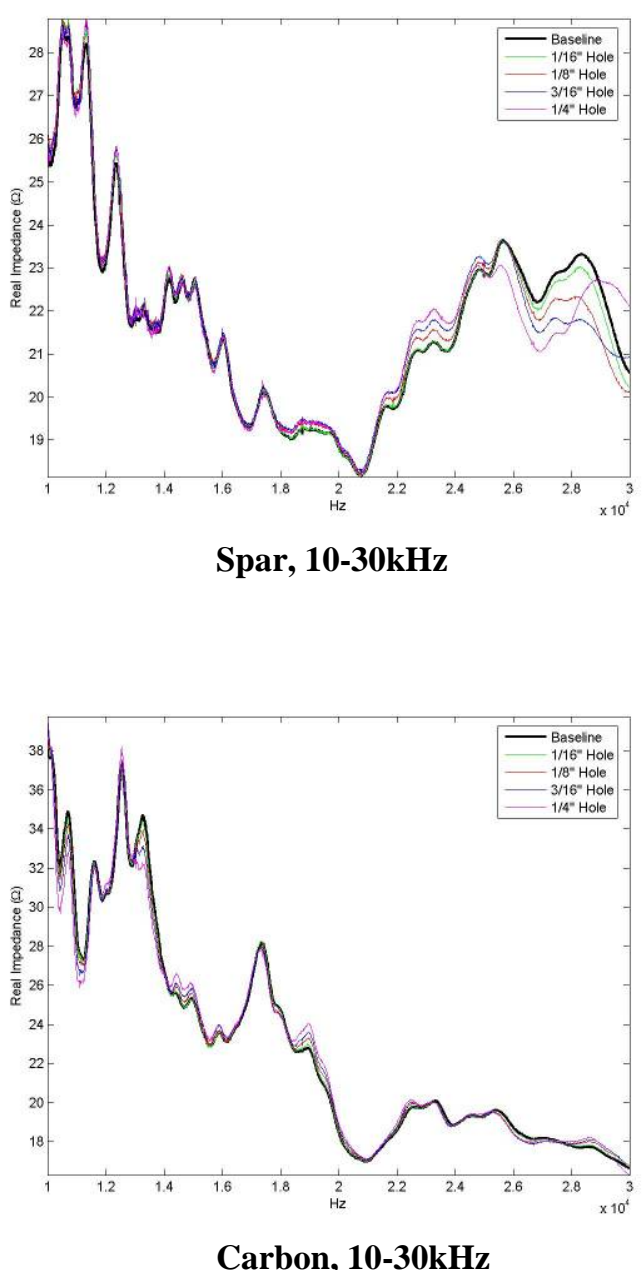

Carbon, 10-30kHz

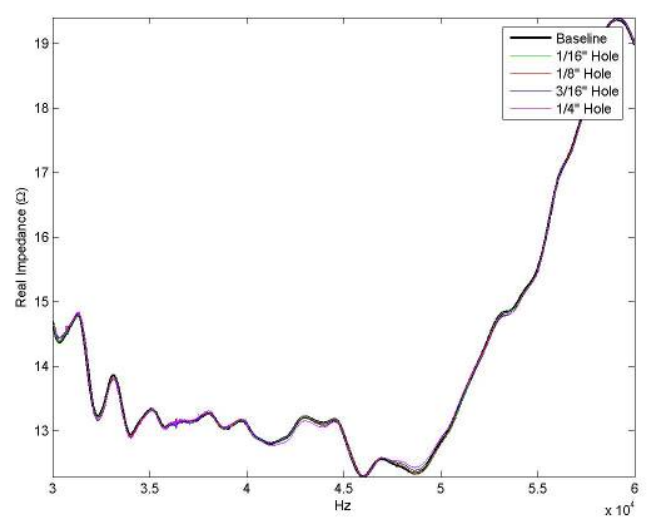

Skin, $30-60 \mathrm{kHz}$

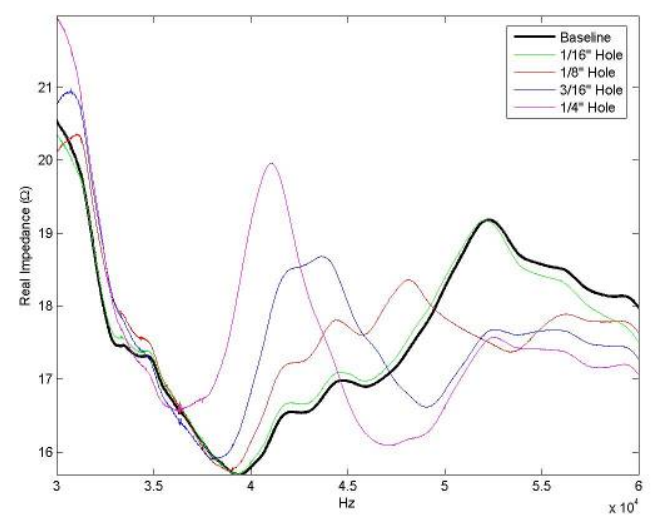

Spar, $30-60 k H z$

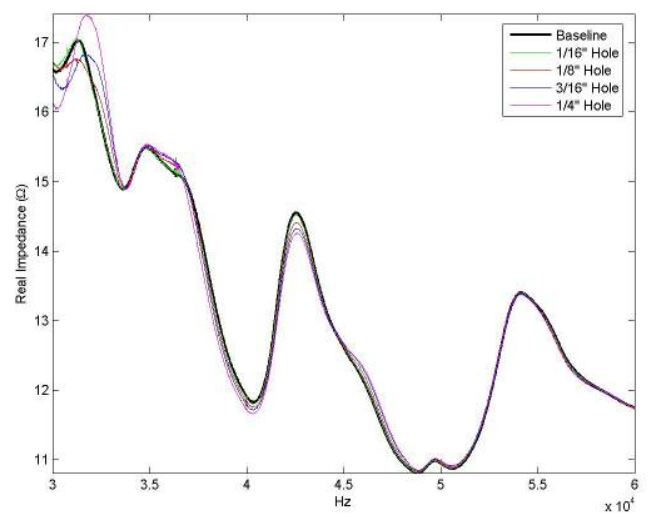

Carbon, 30-60kHz 


\section{APPENDIX C: HFRF, indirect damage, transfer functions}

HFRF baselines with coherence
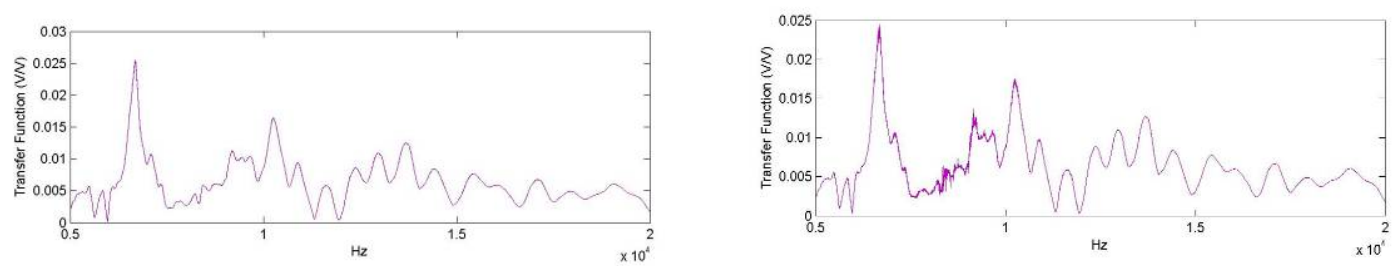

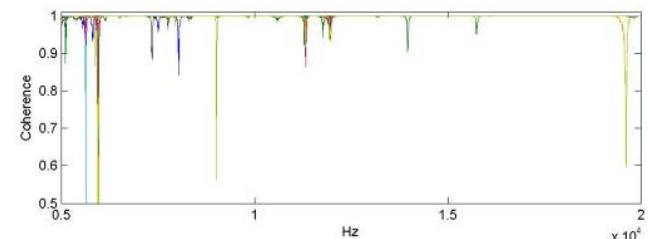

Skin/carbon 1v
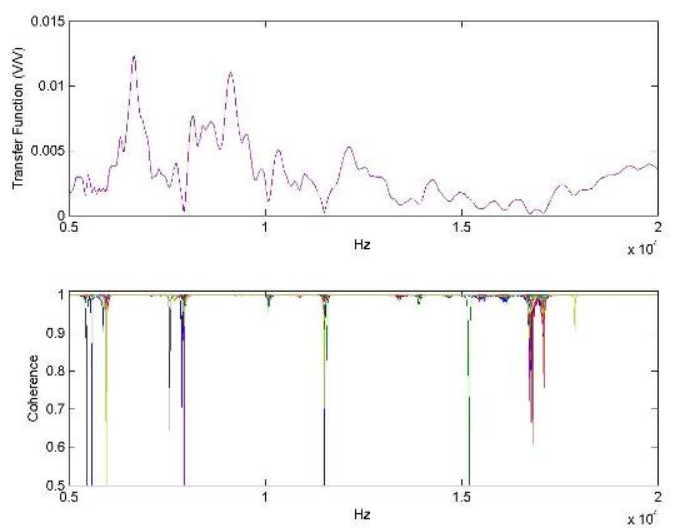

Skin/spar 1v
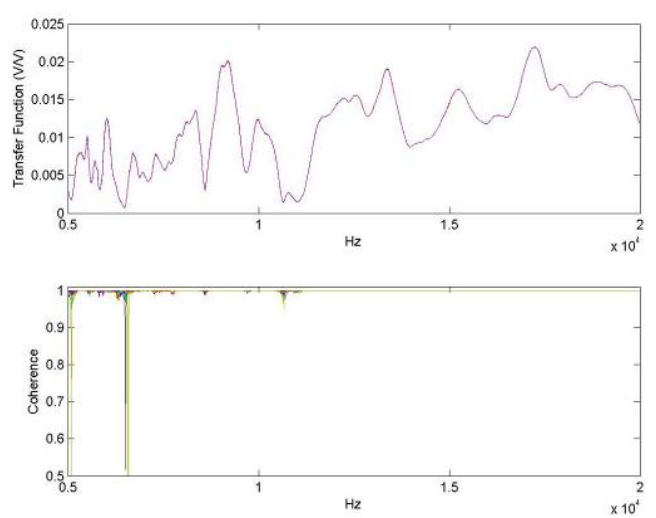

Spar/carbon 1v

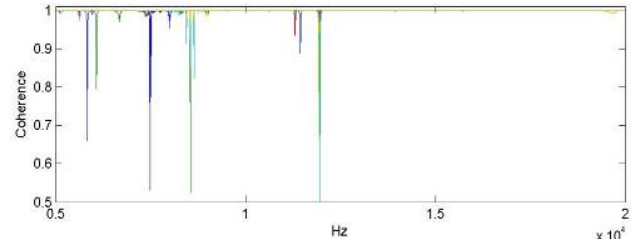

Skin/carbon 5v
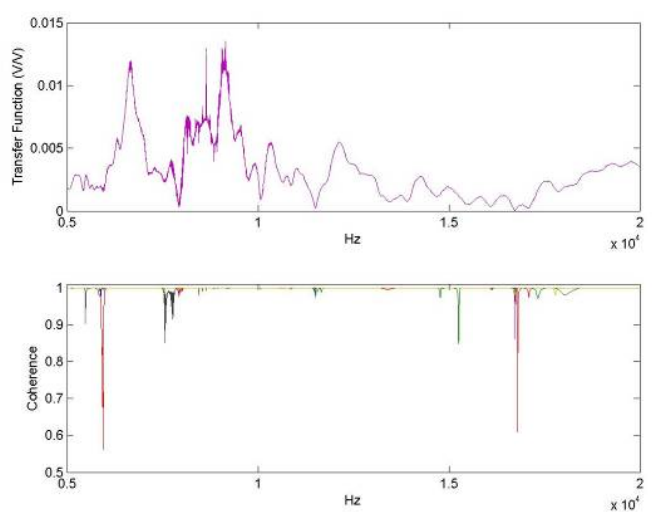

Skin/spar 5v
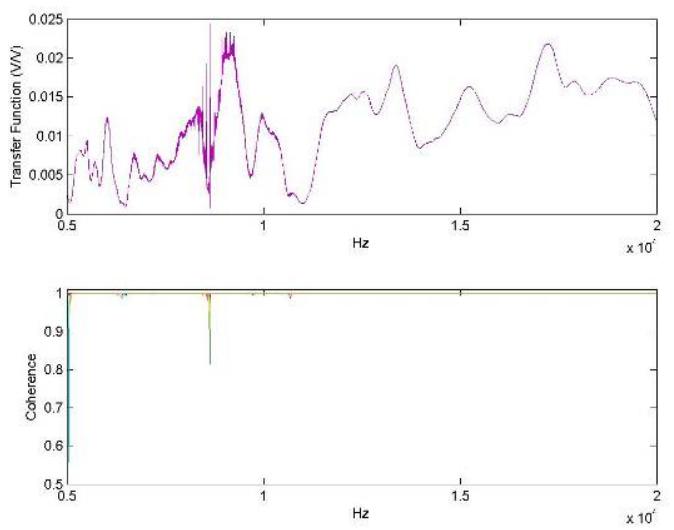

Spar/carbon 5v 


\section{Added mass}
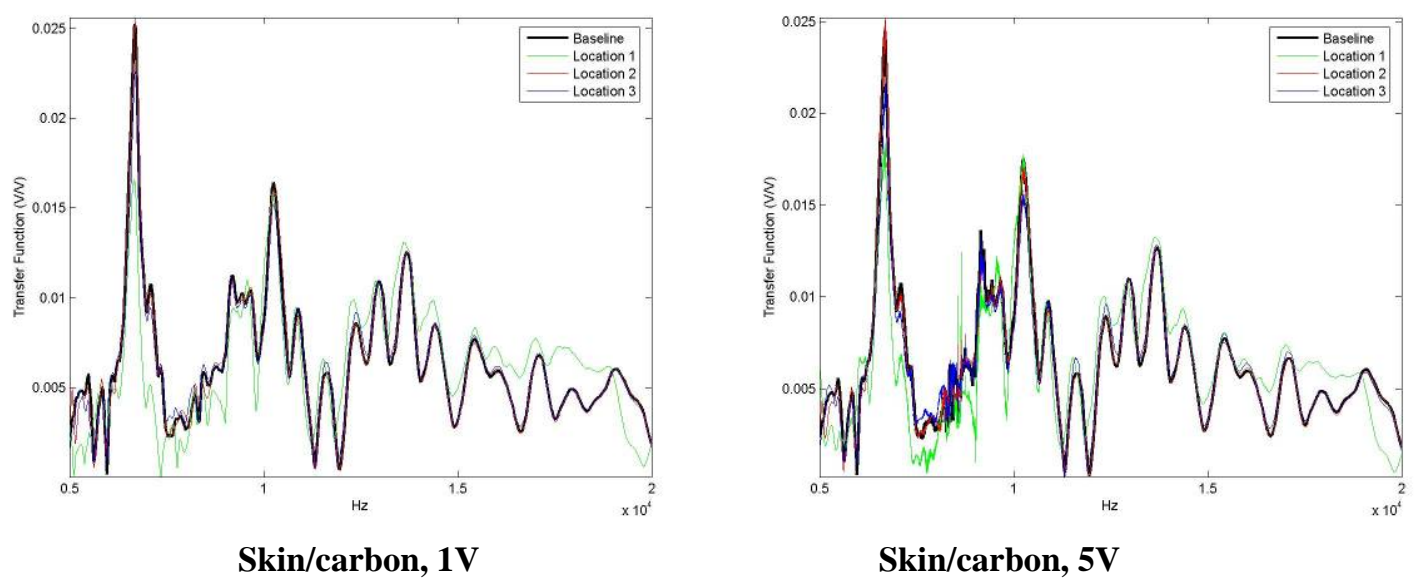

Skin/carbon, 5V

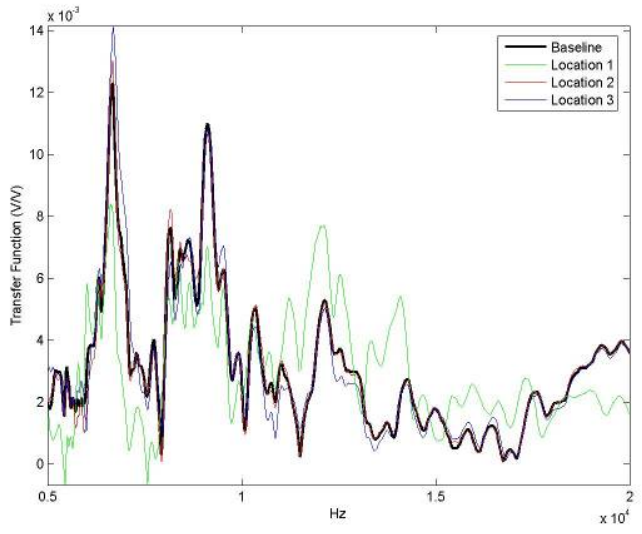

Skin/spar, 1V

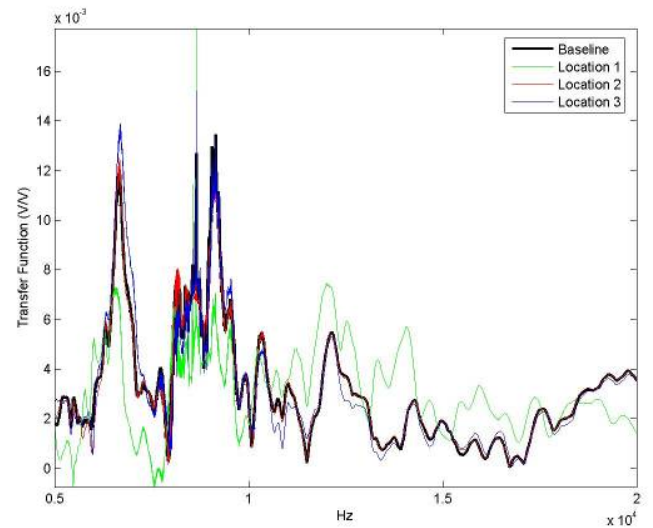

Skin/spar, 5V
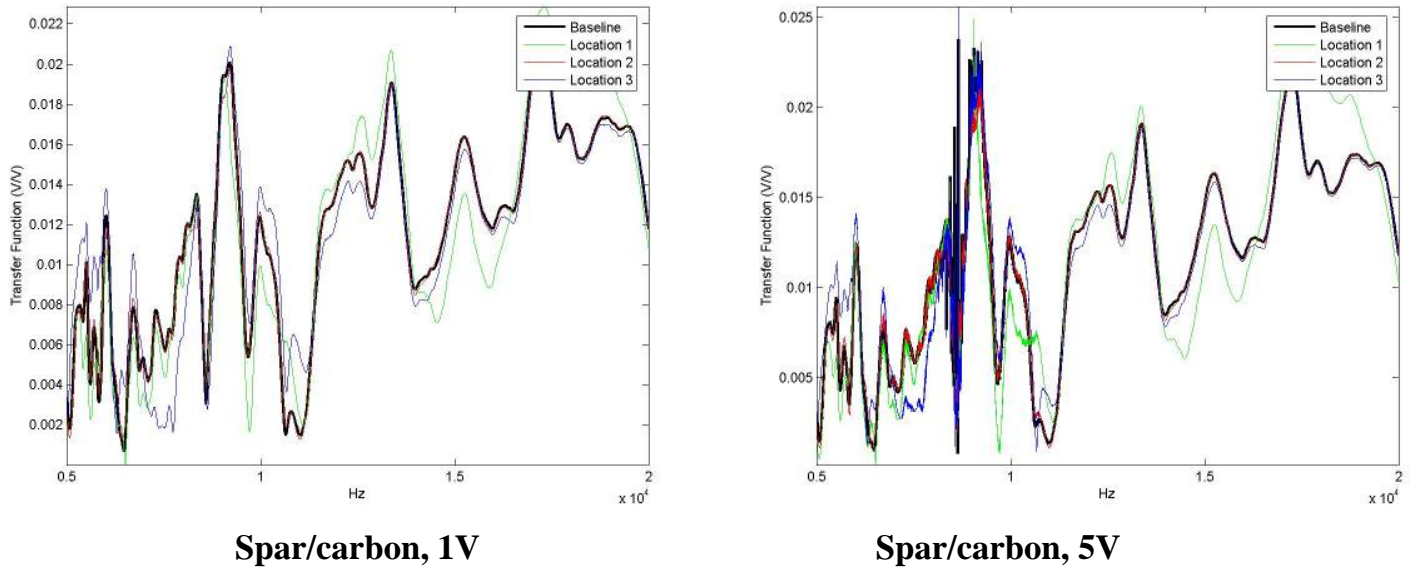


\section{Added stiffness}
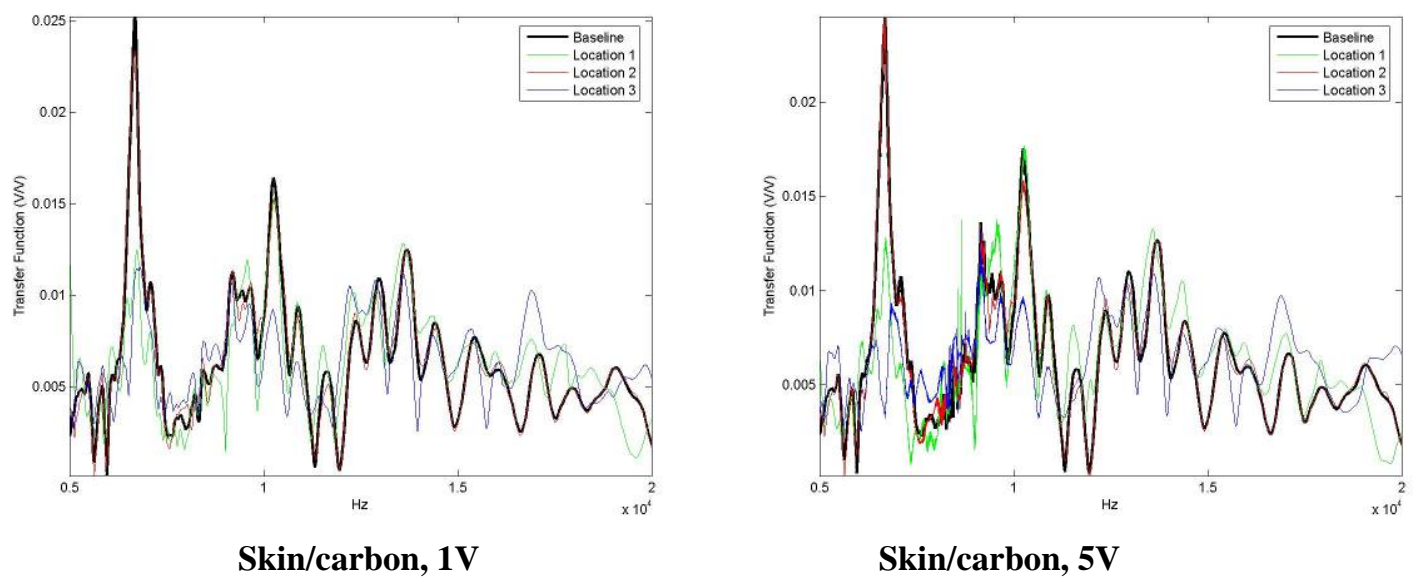

Skin/carbon, 5V

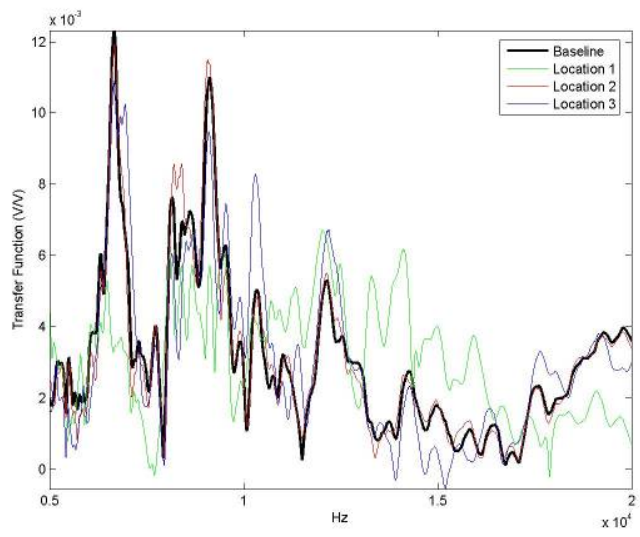

Skin/spar, 1V

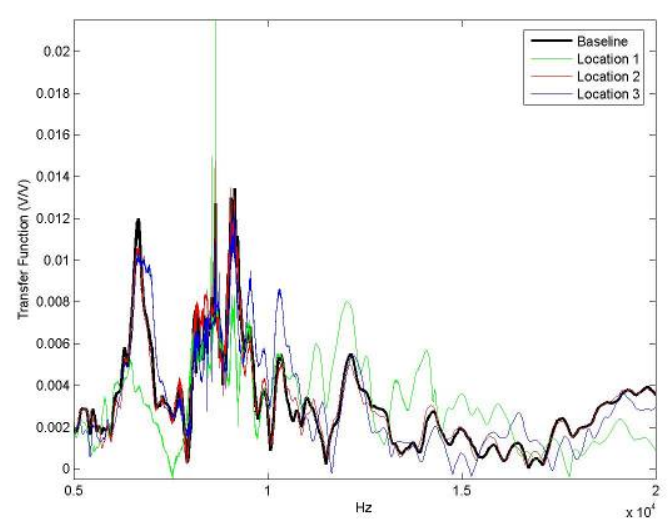

Skin/spar, 5V
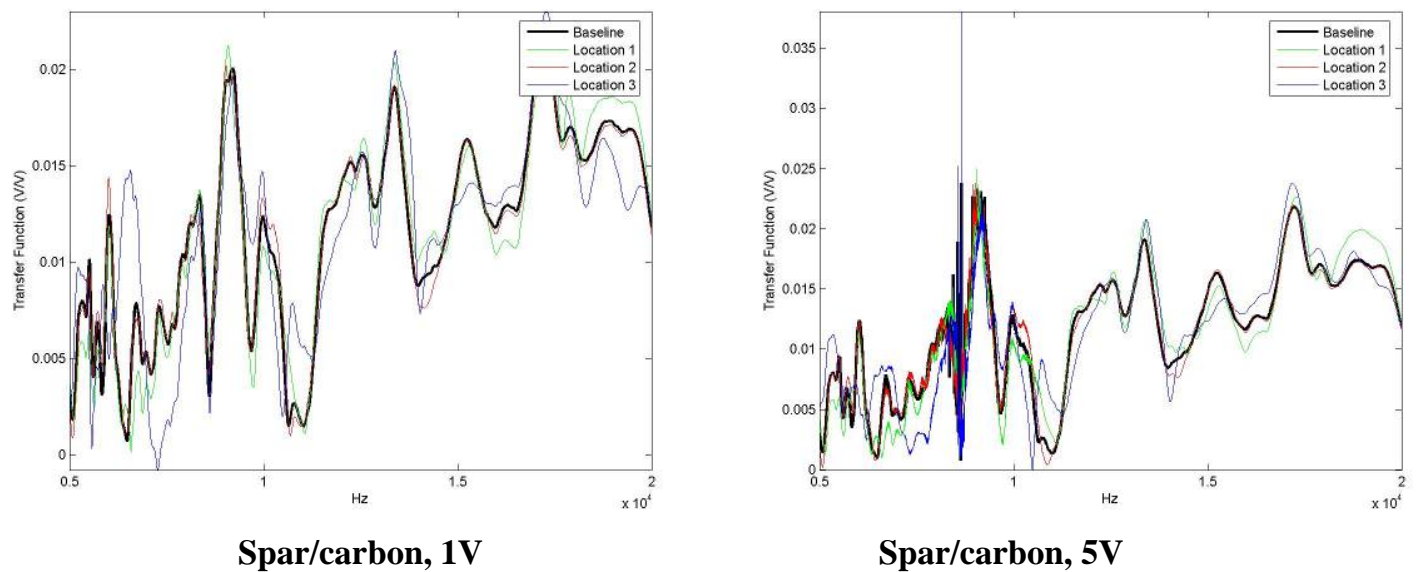


\section{APPENDIX D: HFRF, actual damage, transfer functions}

\section{Location 1}

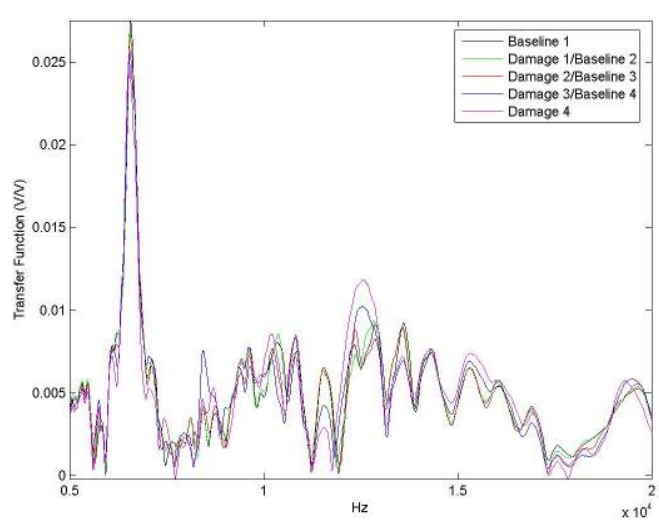

Skin/carbon, down blade

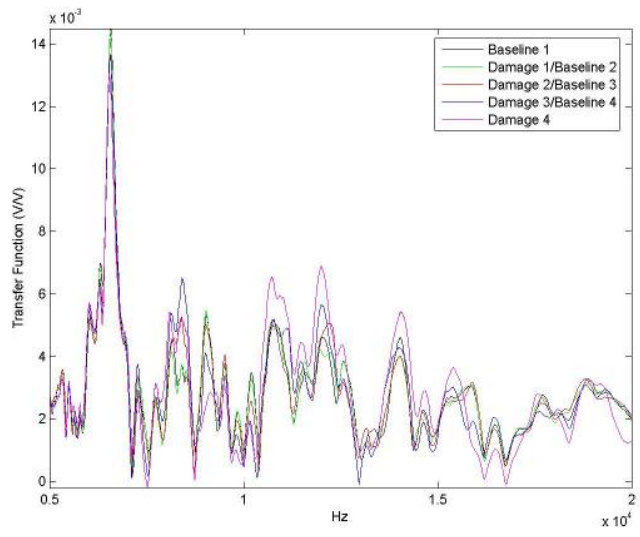

Skin/spar, down blade

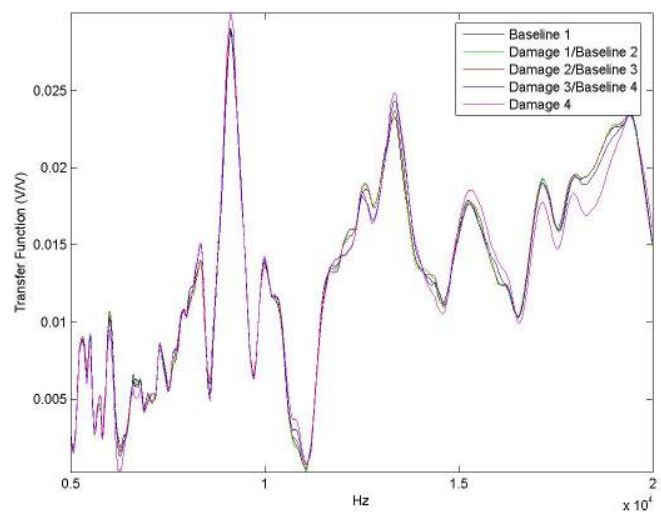

Spar/carbon, down blade

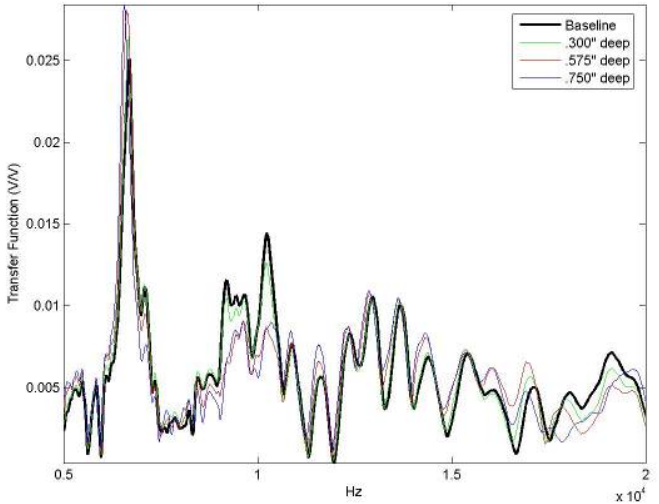

Skin/carbon, split

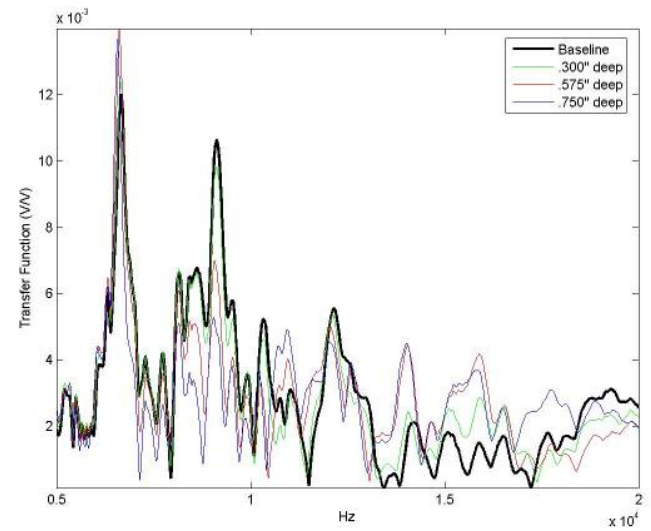

Skin/spar, split

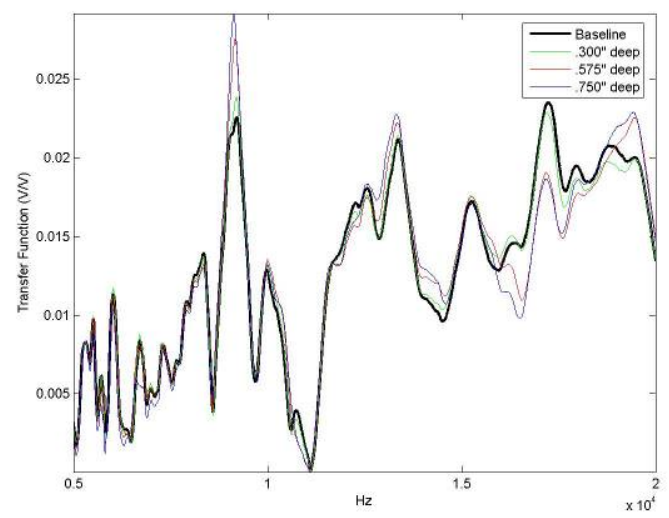

Spar/carbon, split 


\section{Location 3}

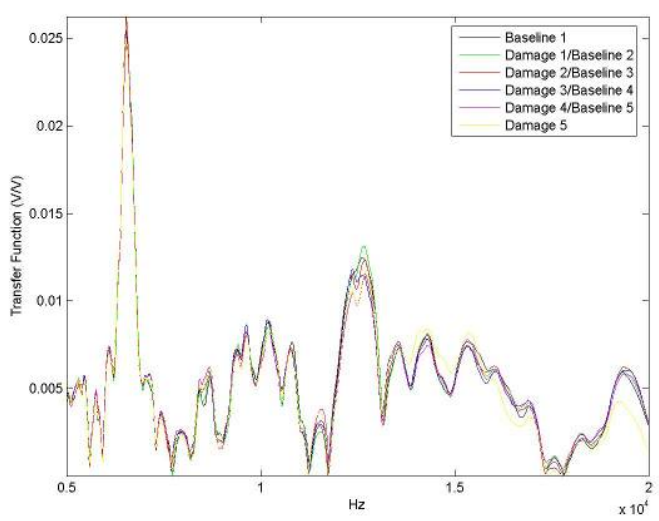

Skin/carbon, down blade

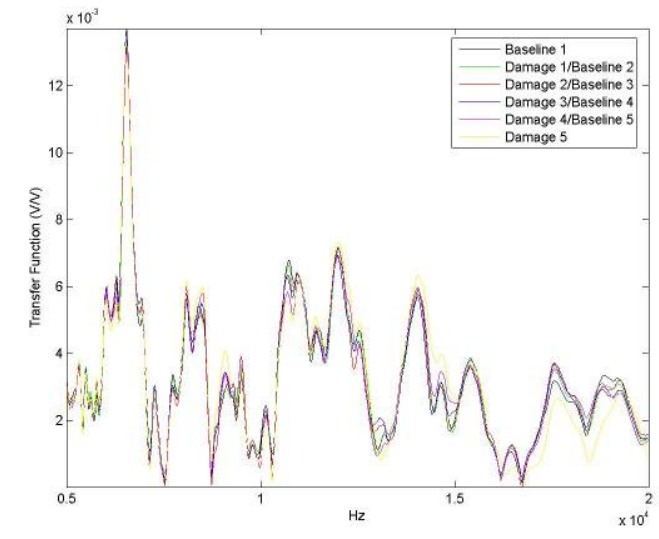

Skin/spar, down blade

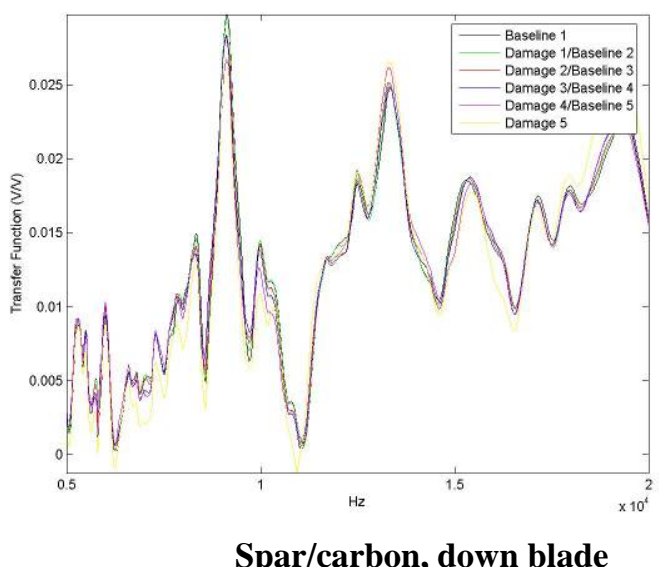

Spar/carbon, down blade

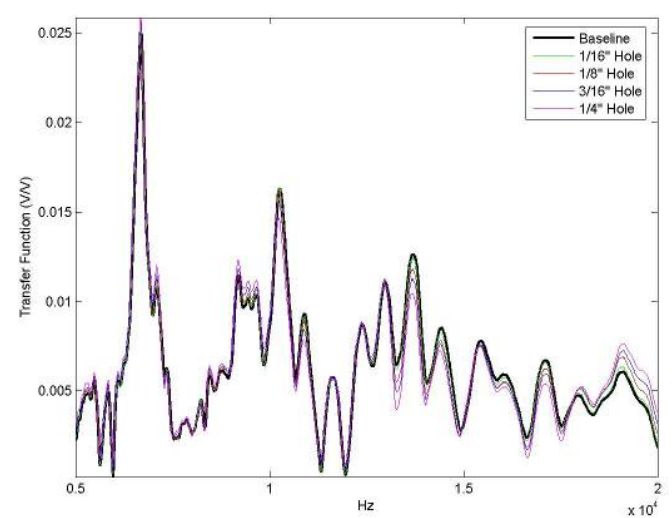

Skin/carbon, holes

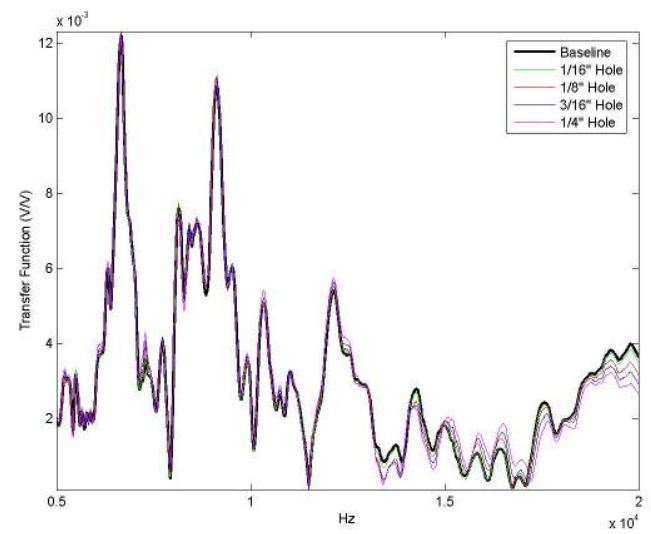

Skin/spar, holes

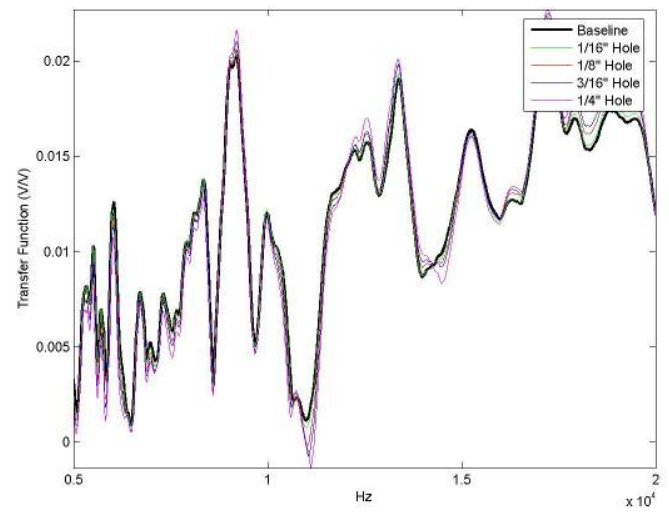

Spar/carbon, holes 


\section{APPENDIX E: HP4194A/HP4192A comparison, impedance plots}

\section{Low Pressure Side}

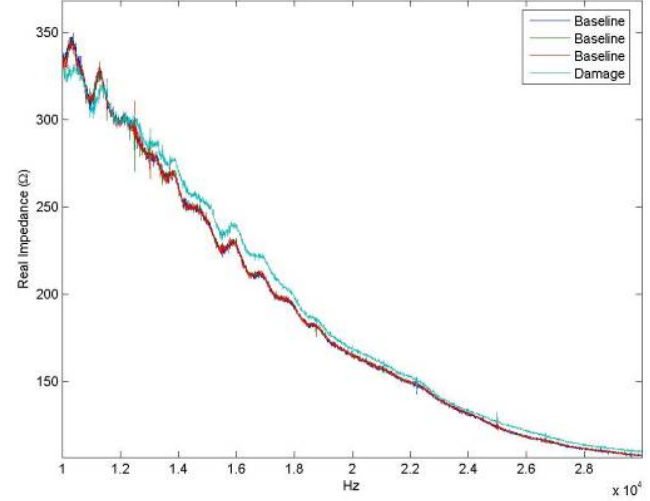

Outer, HP4194A

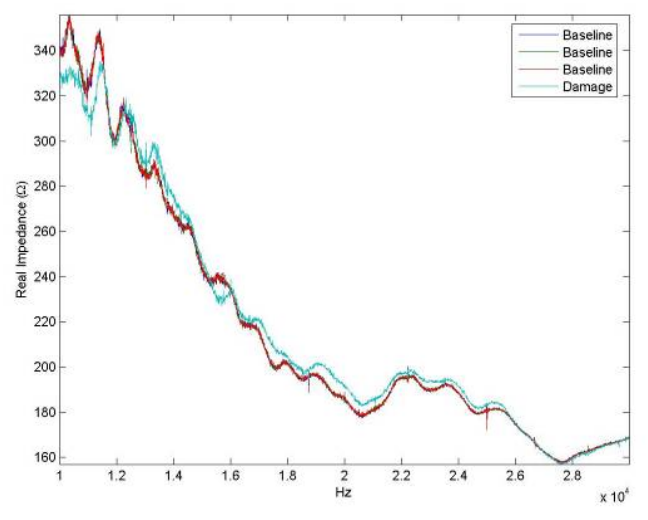

Inner, HP4194A

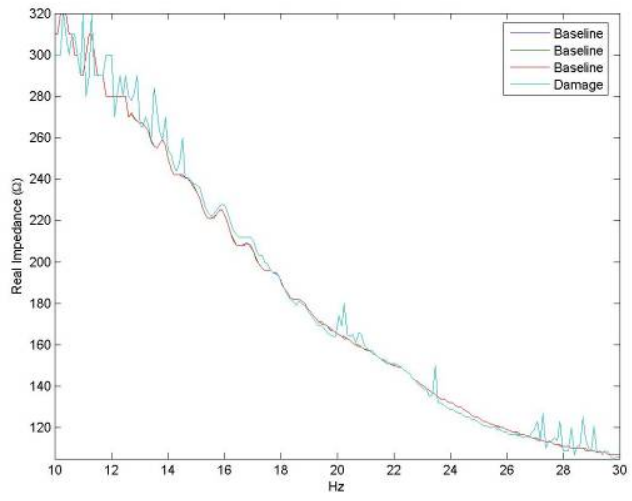

Outer, HP4192A

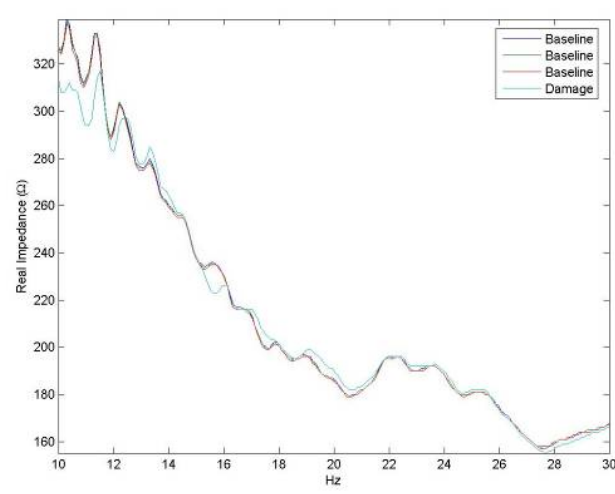

Inner, HP4192A 


\section{High Pressure Side}

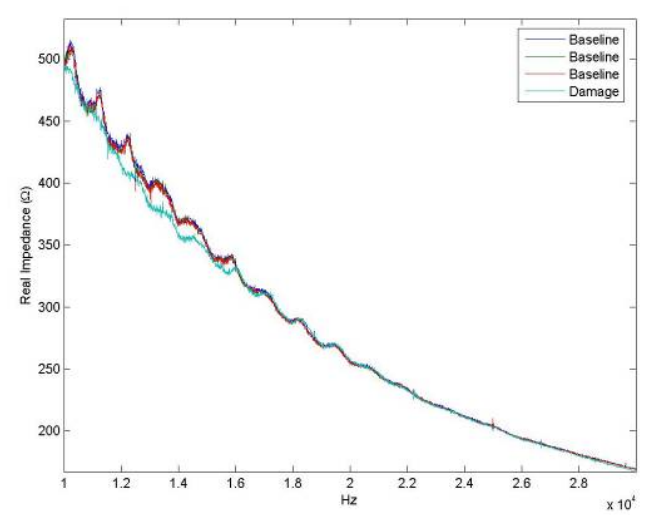

Outer, HP4194A

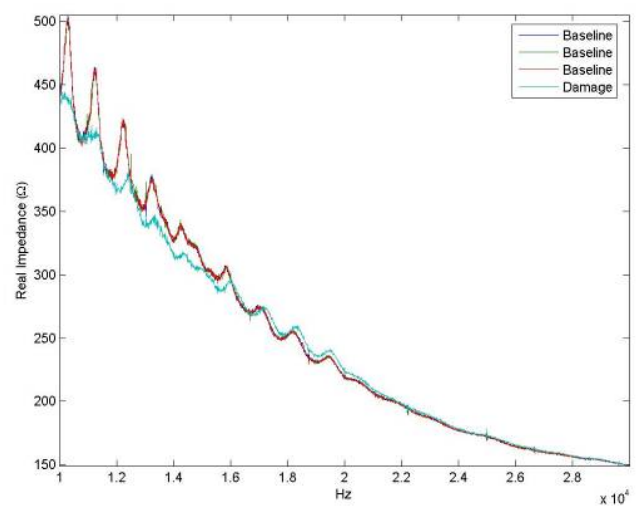

Inner, HP4194A

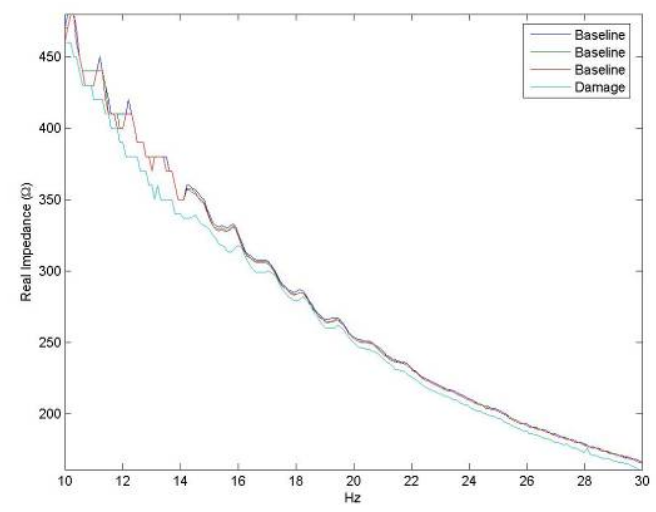

Outer, HP4192A

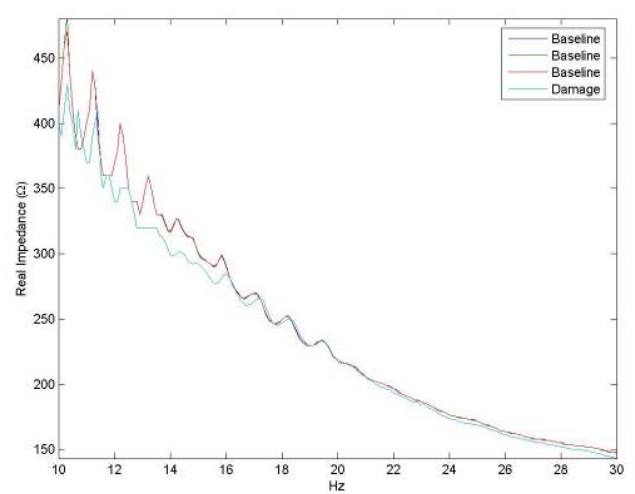

Inner, HP4192A 


\section{APPENDIX F: Fatigue test initial impedance plots}

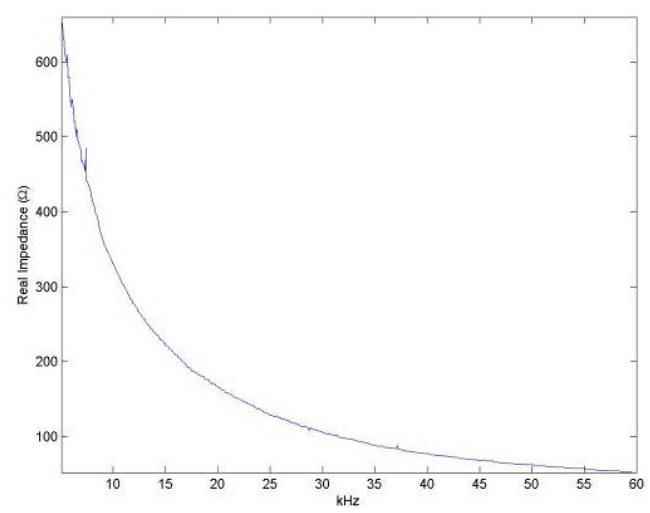

S1

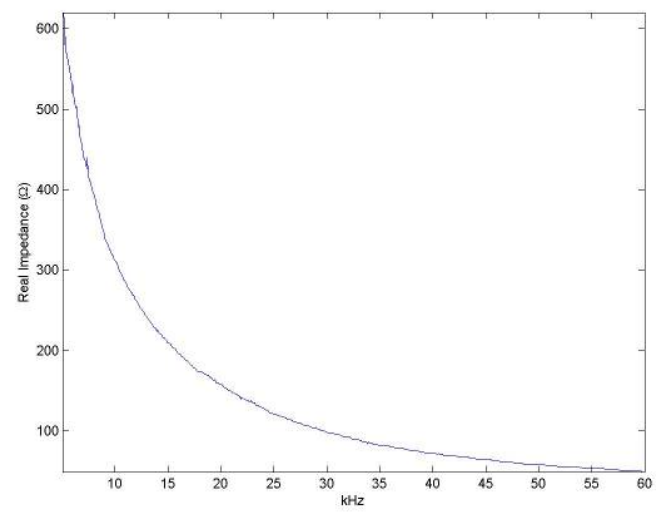

S3

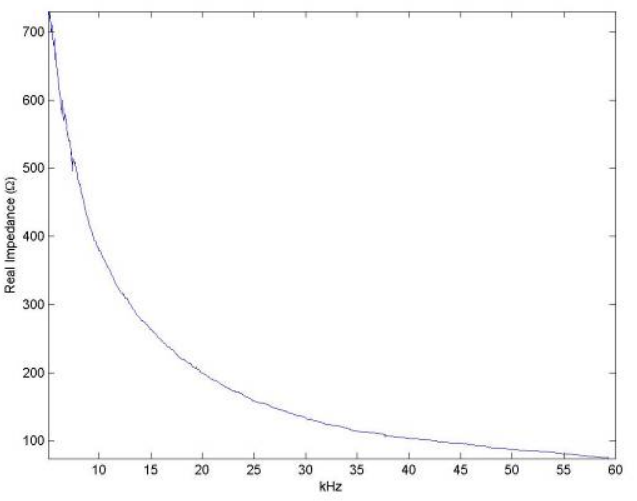

GC1

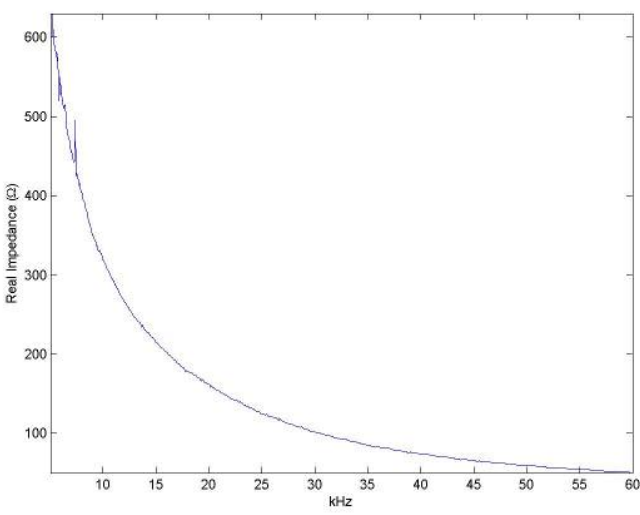

S2

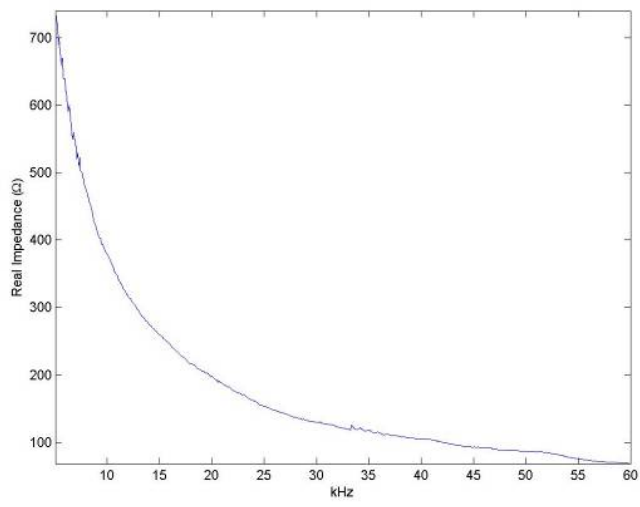

S4

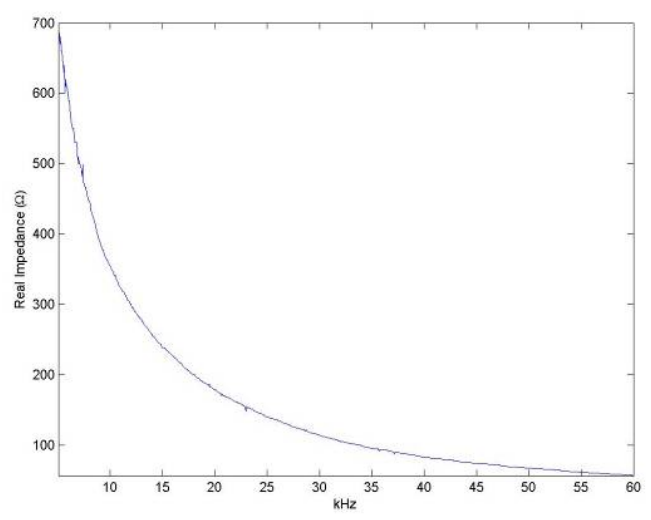

GC2 


\section{APPENDIX G: Data collection instructions for TX-100 fatigue test impedance based SHM}

- Turn on power to HP4192A (LINE button on upper left front panel) and to laptop. Start PRAP software on laptop.

o This step is only necessary if the power has been shut down to these items. In general, it's okay to just leave them running.

- Please take data whenever the fatigue test is stopped for inspection/maintenance or at least once a day during the week.

- Be sure that no other tests (AE, Rudy, Jon) are running and that the blade has come to a stop. Try not to disturb the blade while taking impedance sweeps.

- Connect the BNC connector from the probe fixture to the switch box. This should be disconnected when the impedance analyzer is not in use to insure that no signal is being sent to the MFCs which would disturb other tests.

- Put the selector switch in the position for the desired MFC.

o s1-s4 correspond to spar 1-4 sensors and gc1-gc2 correspond to glass/carbon transition sensors

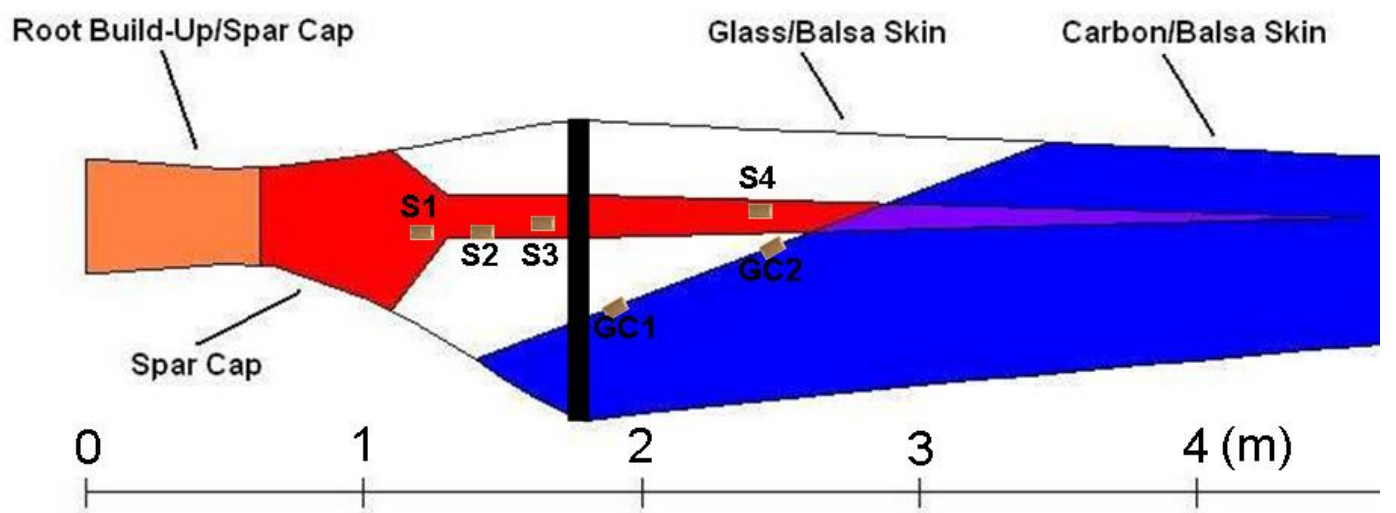

- Click on the red spectrum button ("acquire spectrum") in PRAP

- This will bring up a dialog box. The information here should be the same for each run, but please confirm that the information is correct and if it is not change it before clicking okay.

o $\quad$ Start $(\mathrm{kHz}): 5$

O $\quad$ Stop $(\mathrm{kHz}): 25$

o Number of points: 200

o AC Signal (V): 1

O DC bias (V): 0

o Measure Mode: $\mathrm{R}$ and $\mathrm{X}$

- Stress $(\mathrm{Pa}): 0$ (doesn't really matter)

o Temperature ( $\mathrm{deg} C): 25$ (doesn't really matter)

o Speed Options: Averaging

o Log Scan: unchecked

o Reset instrument when done: unchecked

- After confirming the parameters, click okay. This will begin the sweep for the selected MFC. 
- The sweep should take approximately 4 minutes. A dialog box will come up when sweep is finished, click okay.

- Click the disk button to save the run. Save to the TX100_fatigue folder on the desktop. Before saving, select from the dropdown menu "PRAP import resonance files", not "PRAP resonance files".

- Save the file as sensor name_sweep number. Sensor name will be s1, s2, s3, s4, gc1, or gc2. Sweep number will be one more than the last set of sweeps. So one set of sweeps are s1_1, s2_1, s3_1, etc. and the next sweep will be s1_2, s2_2, s3_2, etc.

- After you are certain the run is saved (the box in PRAP with the impedance sweep in it should have the file name as title) close the impedance sweep box.

- Select the next MFC with the selector switch and repeat the sweep process for all six sensors.

- When the sweeps are complete disconnect the BNC connector from the switch box to insure no signal is going to MFCs.

- In the TX100_fatigue folder on the desktop there is a log file, make a new entry for_sweep number and note the date and approximate number of cycles when data was taken and any other pertinent information and save the file.

- Transfer the new log file and 6 sweep files to floppy disk and transfer to http://wind.nrel.gov/TXfatigue. (Make sure to leave a copy of data on laptop hard drive as well) Please transfer the data every day time permitting or every few days otherwise. I would like to be able to keep up with the data as real time as possible.

"Normal" Operation

- While a sweep is running in the 7 or $8 \mathrm{kHz}$ to 15 or $20 \mathrm{kHz}$ range you should be able to hear the MFC from a few feet away. It would be a good idea to check to make sure you can hear it occasionally.

- The real part of the impedance (top plot in PRAP) should be roughly 100-600 Ohms and early in the test looks like an exponentially decaying function. It is likely that this shape will change during the course of the test but impedance range should roughly stay the same. If there is any question as to whether the data is "normal" please contact Corey Pitchford. 


\section{APPENDIX H: Fatigue test impedance plots}

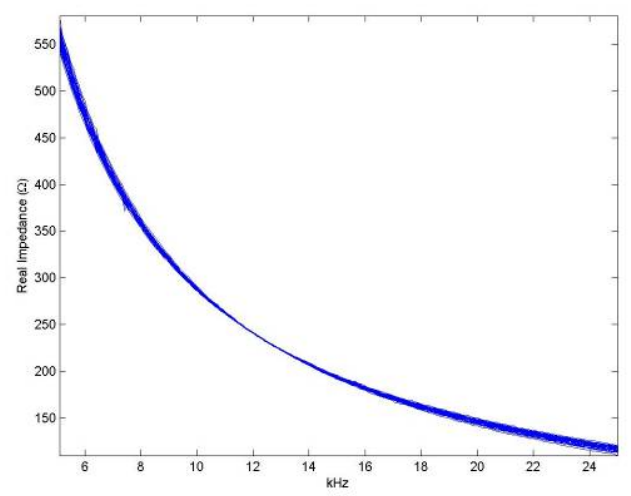

S1

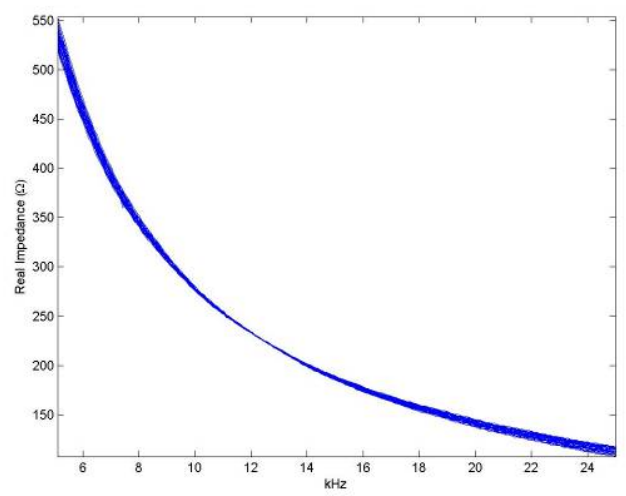

S3

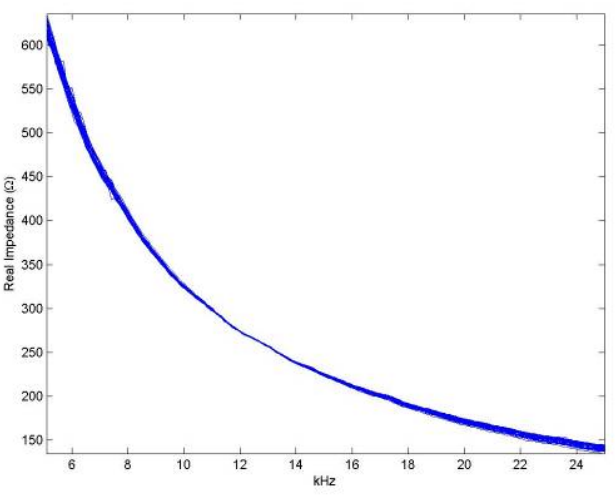

GC1

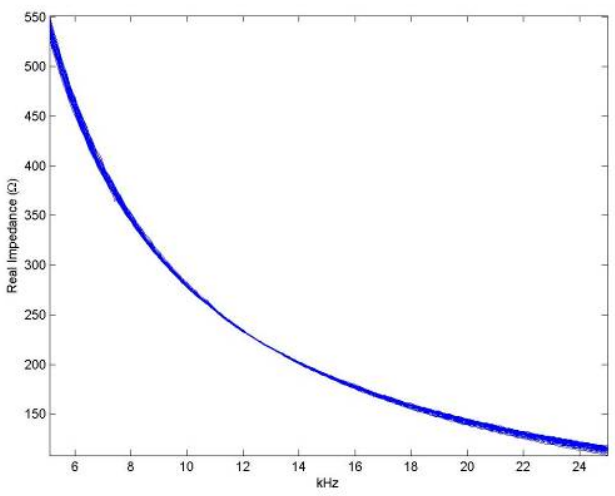

S2

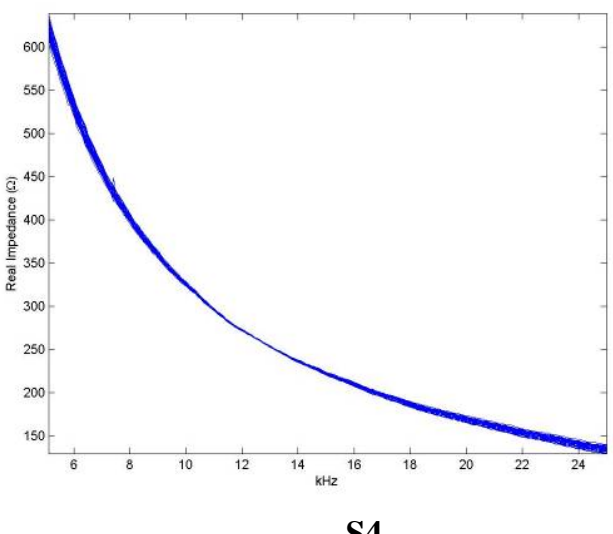

S4

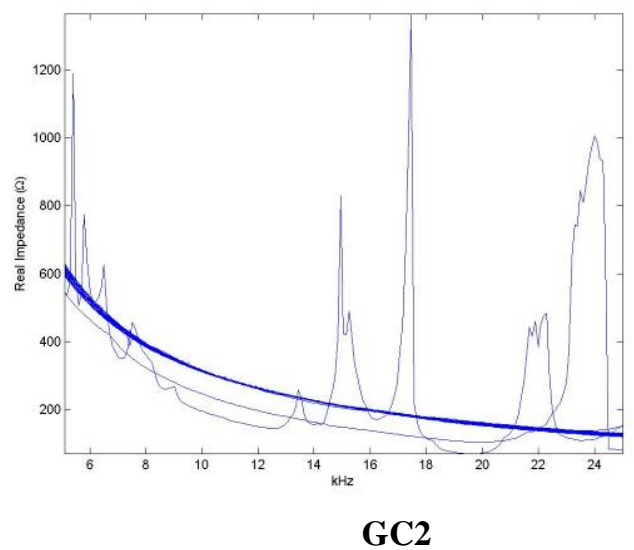




\section{VITA}

Corey Wilson Pitchford was born September 27, 1981 in Springfield, Ohio to Ernie and Ronda Pitchford. He lived in Ohio until the age of thirteen when his family moved to Paris, Tennessee where he graduated from Henry County High School in 2000. In the fall of 2000, Corey began his undergraduate degree from Tennessee Technological University in Cookeville, Tennessee. During his undergraduate studies, Corey was an intern at the Plumley Division of Dana Corporation in Paris, Tennessee, Fleetguard Corporation in Cookeville, Tennessee, and Aerospace Testing Alliance on Arnold Air Force Base, Tennessee. He also worked for DeVilbiss Air Power Company in Jackson, Tennessee as part of a year-long cooperative education program. In 2002, Corey married Melissa Rhea Maggard of Paris, Tennessee. Corey graduated Summa Cum Laude from Tennessee Technological University in May 2005 with a Bachelor's of Science degree in Mechanical Engineering. That fall he started his working on his Master's Degree in Mechanical Engineering at Virginia Polytechnic Institute and State University. During his first year of graduate study, Corey was a teaching assistant for the Mechanical Engineering Lab courses. In summer 2006, he started working in the Center for Intelligent Material Systems and Structures as a research assistant under Daniel J. Inman. His research has focused on investigating structural health monitoring, primarily impedance-based, in wind turbine blades. After defending his thesis, Corey will begin working at Eastman Chemical Company in Kingsport, Tennessee as a mechanical engineer in the Materials, Vessel, and Piping Technology department focusing on pressure vessel and finite element analysis.

\section{Address:}

622 Fordtown Road

Kingsport, Tennessee 37663 